\title{
An updated checklist of the vascular flora native to Italy
}

F. Bartolucci, L. Peruzzi, G. Galasso, A. Albano, A. Alessandrini, N. M. G. Ardenghi, G. Astuti, G. Bacchetta, S. Ballelli, E. Banfi, G. Barberis, L. Bernardo, D. Bouvet, M. Bovio, L. Cecchi, R. Di Pietro, G. Domina, S. Fascetti, G. Fenu, F. Festi, B. Foggi, L. Gallo, G. Gottschlich, L. Gubellini, D. Iamonico, M. Iberite, P. Jiménez-Mejías, E. Lattanzi, D. Marchetti, E. Martinetto, R. R. Masin, P. Medagli, N. G. Passalacqua, S. Peccenini, R. Pennesi, B. Pierini, L. Poldini, F. Prosser, F. M. Raimondo, F. Roma-Marzio, L. Rosati, A. Santangelo, A. Scoppola, S. Scortegagna, A. Selvaggi, F. Selvi, A. Soldano, A. Stinca, R. P. Wagensommer, T. Wilhalm \& F. Conti

To cite this article: F. Bartolucci, L. Peruzzi, G. Galasso, A. Albano, A. Alessandrini, N. M. G. Ardenghi, G. Astuti, G. Bacchetta, S. Ballelli, E. Banfi, G. Barberis, L. Bernardo, D. Bouvet, M. Bovio, L. Cecchi, R. Di Pietro, G. Domina, S. Fascetti, G. Fenu, F. Festi, B. Foggi, L. Gallo, G. Gottschlich, L. Gubellini, D. lamonico, M. Iberite, P. Jiménez-Mejías, E. Lattanzi, D. Marchetti, E. Martinetto, R. R. Masin, P. Medagli, N. G. Passalacqua, S. Peccenini, R. Pennesi, B. Pierini, L. Poldini, F. Prosser, F. M. Raimondo, F. Roma-Marzio, L. Rosati, A. Santangelo, A. Scoppola, S. Scortegagna, A. Selvaggi, F. Selvi, A. Soldano, A. Stinca, R. P. Wagensommer, T. Wilhalm \& F. Conti (2018) An updated checklist of the vascular flora native to Italy, Plant Biosystems - An International Journal Dealing with all Aspects of Plant Biology, 152:2, 179-303

To link to this article: https://doi.org/10.1080/11263504.2017.1419996

View supplementary material $\asymp$

Published online: 08 Feb 2018.

Submit your article to this journal $₫$

View related articles $₫$

View Crossmark data \lceil

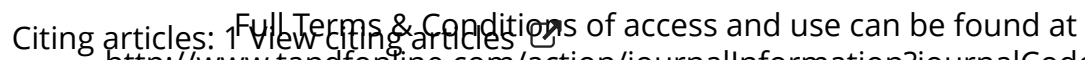
http://www.tandfonfine.com/action/journallnformation?journalCode=tplb20 


\title{
An updated checklist of the vascular flora native to Italy
}

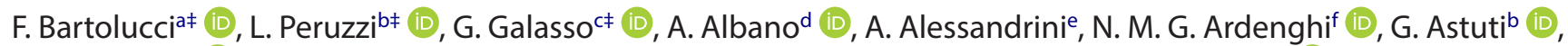 \\ G. Bacchetta9 (D), S. Ballelli', E. Banfic, G. Barberis', L. Bernardoj, D. Bouvet', M. Bovio', L. Cecchim (D), R. Di Pietron,

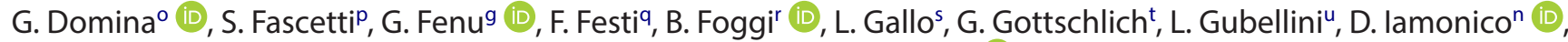

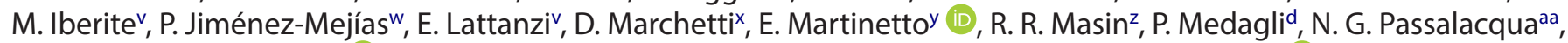

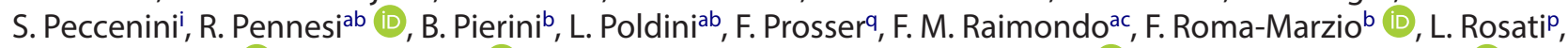 \\ A. Santangeload (D) A. Scoppola ae (D) S. Scortegagnaaf, A. Selvaggiag, F. Selvi id (D), A. Soldano ai, A. Stinca ${ }^{\text {aj }}$, \\ R. P. Wagensommer ${ }^{\text {ak }} \mathbb{D}$, T. Wilhalm ${ }^{\text {al }}$ and F. Contia ${ }^{\text {a }}$
}

aScuola di Bioscienze e Medicina Veterinaria, Università di Camerino - Centro Ricerche Floristiche dell'Appennino, Parco Nazionale del Gran Sasso e Monti della Laga, L'Aquila, Italy; 'Dipartimento di Biologia, Unità di Botanica, Università di Pisa, Pisa, Italy; ' Sezione di Botanica, Museo di Storia Naturale

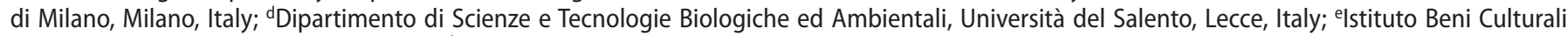
Regione Emilia-Romagna, Bologna, Italy; ${ }^{\mathrm{f} D i p a r t i m e n t o ~ d i ~ S c i e n z e ~ d e l l a ~ T e r r a ~ e ~ d e l l ' A m b i e n t e, ~ U n i v e r s i t a ̀ ~ d i ~ P a v i a, ~ P a v i a, ~ I t a l y ; ~}{ }^{9} \mathrm{Centro}$ Conservazione

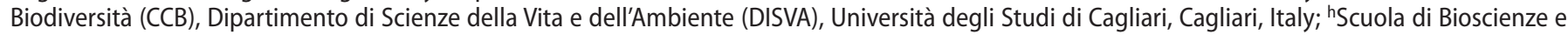
Medicina Veterinaria, Università di Camerino, Camerino, Italy; 'Dipartimento di Scienze della Terra, dell'Ambiente e della Vita, Università di Genova, Genova, Italy; 'Dipartimento DiBEST, Università della Calabria, Cosenza, Italy; ${ }^{\circ}$ Dipartimento di Scienze della Vita e Biologia dei Sistemi (DBIOS), Università di Torino, Torino, Italy; 'Comitato Scientifico, Museo Regionale di Scienze Naturali "Efisio Noussan", Aosta, Italy; mSezione di Botanica "Filippo Parlatore", Università degli Studi di Firenze, Museo di Storia Naturale, Firenze, Italy; "Dipartimento PDTA, Università di Roma "La Sapienza", Roma,

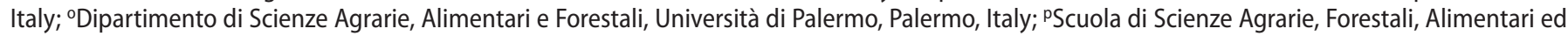
Ambientali, Università della Basilicata, Potenza, Italy; ${ }^{9}$ Fondazione Museo Civico di Rovereto, Trento, Italy; 'Dipartimento di Biologia, Università di Firenze,

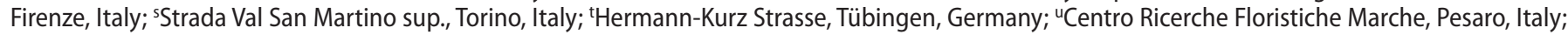
"Dipartimento di Biologia ambientale, Università di Roma "La Sapienza", Roma, Italy; "National Museum of Natural History, Smithsonian Institution,

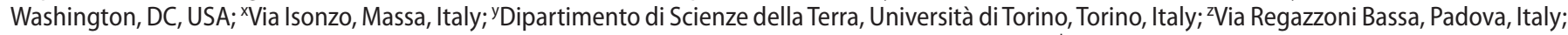
${ }^{a}$ Museo di Storia Naturale della Calabria ed Orto Botanico, Università della Calabria, Cosenza, Italy; abDipartimento di Scienze della Vita, Università di Trieste, Trieste, Italy; ${ }^{a}$ Dipartimento STEBICEF/Sezione di Botanica ed Ecologia vegetale, Università di Palermo, Palermo, Italy; adDiparimento di Biologia,

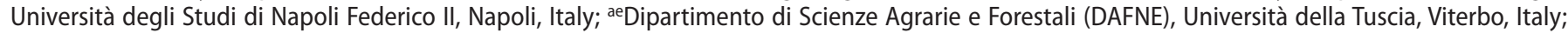
afVia Europa Unita, Schio, Italy; ${ }^{a g}$ Istituto per le Piante da Legno e I'Ambiente (IPLA), Torino, Italy; ahLaboratori di Botanica, Dipartimento di Scienze

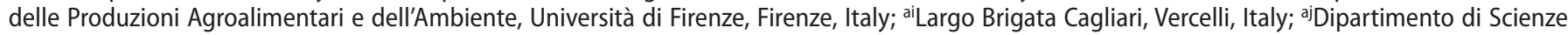

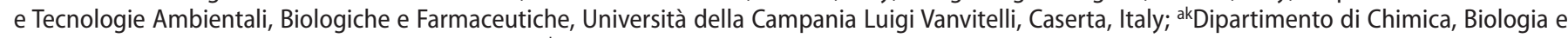

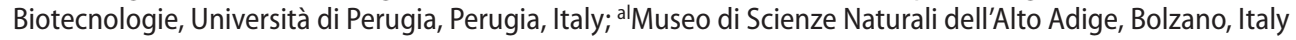

\section{ABSTRACT}

An updated inventory of the native vascular flora of Italy, providing details on the occurrence at regional level, is presented. The checklist includes 8195 taxa (6417 species and 1778 subspecies), distributed in 1092 genera and 152 families; 23 taxa are lycophytes, 108 ferns and fern allies, 30 gymnosperms and 8034 angiosperms. The taxa currently occurring in Italy are 7483, while 568 taxa have not been confirmed in recent times, 99 are doubtfully occurring in the country and 19 are data deficient. Out of the 568 not confirmed taxa, 26 are considered extinct or possibly extinct.

\section{ARTICLE HISTORY}

Received 28 November 2017 Accepted 19 December 2017

\section{KEYWORDS}

Endemism; floristic diversity; Italy; Mediterranean flora; taxonomy

\section{Introduction}

The last comprehensive checklist of the Italian vascular flora of native (including archaeophytes) and non-native established taxa was published 12 years ago, and included 7634 specific and subspecific taxa, of which 782 non-native (Conti et al. 2005), albeit these numbers were slightly modified by Conti et al. (2007). More recently, an inventory of alien (casual, naturalized and invasive taxa) and cryptogenic vascular flora to Italy, including archaeophytes and neophytes, listed 1023 species and subspecies, of which 103 archaeophytes and 920 neophytes (Celesti-Grapow et al. 2009).

However, in the last years, there has been substantial progress in systematics, taxonomy, nomenclature and alien plant invasion research. Several new floristic and taxonomic data for Italy have been published in regional floras, local floristic contributions, taxonomic reviews and in the scientific reports published by the working group for Floristics, Systematics and Evolution of the Italian Botanical Society (e.g. Alessandrini et al. 2017; Bartolucci et

CONTACT F. Bartolucci fabrizio.bartolucci@unicam.it, fabrizio.bartolucci@gmail.com

₹These authors equally contributed to the paper

(1) Supplemental data for this article can be accessed at https://doi.org/10.1080/11263504.2017.1419996

○ 2018 Società Botanica Italiana 
al. 2017a, 2017b; Rosati et al. 2017). Furthermore, many taxa have been recently described for Italy as new to science (e.g. Bacchetta et al. 2012a, 2012b; Melai et al. 2012; Peruzzi and Gestri 2013; Troìa and Azzella 2013; Peccenini and Polatschek 2014; Domina and Soldano 2015; Brullo et al. 2015b; Peccenini and Polatschek 2016; Conti and Bartolucci 2017). Therefore, there is a need of a new checklist to summarize the current state of the floristic and taxonomic knowledge of the Italian vascular flora.

In this context, we thought appropriate to publish two separate inventories: the present work, concerning only the native (including cryptogenic or doubfully native) vascular flora (Bartolucci et al. 2016c), and a second one concerning only the alien taxa (archaeophytes and neophytes) (Galasso et al. 2016a).

This checklist is the result of a collective effort, thanks to the voluntary collaboration of many researchers who, as regional or taxonomic advisers (see Appendix S1 in supplementary material for details), have contributed to filling gaps in distribution or clarifying systematic and nomenclatural issues. This also served as a basis for an inventory of endemic taxa of Italy (Peruzzi et al. 2014a, 2015a), for the project "Italian Loci Classici Census" (Domina et al. 2012; Passalacqua et al. 2014; Peruzzi et al. 2015b; Brundu et al. 2017), for the revised Red List of Italian vascular plants (Rossi et al. 2013, 2014, 2016) and for the updated checklist of the Italian plants listed in the Habitat Directive (Fenu et al. 2017).

The aim of this contribution is to provide an updated inventory of the native vascular flora of Italy, to serve as a taxonomic and nomenclatural basis for botanical research and to promote new floristic investigations and biosystematic studies.

\section{Materials and methods}

Starting from "An annotated checklist of the Italian vascular flora" (Conti et al. 2005, 2007), the nomenclature of specific and subspecific taxa was updated and critically checked according to more recent studies. In addition, numerous protologues and the following taxonomic databases were consulted: IPNI (2012 onwards), The Plant List (2013 onwards), Euro+Med (2006 onwards) and Tropicos (2017).

The systematic order and taxonomic circumscription of the families follow the classification proposed by PPG I (2016) for ferns and fern allies, by Christenhusz et al. (2011) for gymnosperms and by APG IV (2016) for angiosperms, with the exception of Dipsacales (Reveal 2011), Caryophyllales (Hernández-Ledesma et al. 2015) and Boraginales (Luebert et al. 2016). The author citations of plant names were standardized following the Rec. 46A Note 1 of the ICN (McNeill et al. 2012), i.e. according to IPNI (2012 onwards). The checklist includes also apomictic taxa belonging to Alchemilla and Rubus (Rosaceae), Hieracium, Pilosella and Taraxacum (Asteraceae), and Ranunculus auricomus group (Ranunculaceae). Taxa at varietal rank and hybrids were not considered.

The distribution of specific and subspecific taxa at regional scale was updated based on new data published in the period 2005-2015 in the column "Notulae alla checklist della flora vascolare italiana" (Informatore Botanico Italiano), which recently became "Notulae to the Italian native vascular Flora" (Bartolucci
Table 1. Most represented families ( $\geq 50$ taxa) and genera ( $\geq 40$ taxa) of the Italian native vascular flora.

\begin{tabular}{lrlr}
\hline Families & Taxa & Genera & Taxa \\
\hline Asteraceae & 2202 & Hieracium & 1167 \\
Poaceae & 550 & Taraxacum & 154 \\
Fabaceae & 495 & Carex & 126 \\
Caryophyllaceae & 359 & Centaurea & 116 \\
Brassicaceae & 333 & Limonium & 111 \\
Rosaceae & 312 & Ranunculus & 110 \\
Apiaceae & 263 & Ophrys & 103 \\
Lamiaceae & 240 & Pilosella & 100 \\
Orchidaceae & 236 & Alchemilla & 95 \\
Ranunculaceae & 231 & Silene & 87 \\
Cyperaceae & 187 & Festuca & 83 \\
Plantaginaceae & 160 & Trifolium & 81 \\
Orobanchaceae & 140 & Allium & 69 \\
Plumbaginaceae & 135 & Saxifraga & 66 \\
Boraginaceae & 115 & Euphorbia & 62 \\
Campanulaceae & 99 & Galium & 59 \\
Rubiaceae & 95 & Campanula & 58 \\
Amaryllidaceae & 93 & Viola & 56 \\
Primulaceae & 80 & Dianthus & 53 \\
Asparagaceae & 76 & Vicia & 53 \\
Juncaceae & 71 & Astragalus & 45 \\
Saxifragaceae & 69 & Genista & 45 \\
Gentianaceae & 68 & Potentilla & 45 \\
Euphorbiaceae & 67 & Veronica & 44 \\
Chenopodiaceae & 62 & Crepis & 43 \\
Dipsacaceae & 62 & Salix & 43 \\
Crassulaceae & 50 & Rosa & 42 \\
Polygonaceae & 58 & Juncus & 40 \\
Iridaceae & 55 & & 40 \\
Violaceae & & & \\
Cistaceae & 56 & \\
\hline & 53 & \\
\hline
\end{tabular}

Table 2. Most represented families ( $\geq 20$ taxa) and genera ( $\geq 15$ taxa) of the Italian endemic vascular flora.

\begin{tabular}{lclc}
\hline Families & Taxa & \multicolumn{1}{c}{ Genera } & Taxa \\
\hline Asteraceae & 582 & Hieracium & 337 \\
Plumbaginaceae & 117 & Limonium & 98 \\
Caryophyllaceae & 97 & Centaurea & 73 \\
Orchidaceae & 87 & Ophrys & 61 \\
Fabaceae & 80 & Ranunculus & 37 \\
Brassicaceae & 78 & Taraxacum & 33 \\
Poaceae & 65 & Genista & 29 \\
Ranunculaceae & 60 & Silene & 29 \\
Rosaceae & 40 & Dianthus & 28 \\
Apiaceae & 39 & Allium & 25 \\
Lamiaceae & 38 & Viola & 20 \\
Boraginaceae & 34 & Campanula & 19 \\
Amaryllidaceae & 29 & Armeria & 17 \\
Rubiaceae & 28 & Festuca & 17 \\
Campanulaceae & 26 & Saxifraga & 17 \\
Plantaginaceae & 24 & Astragalus & 16 \\
Iridaceae & 23 & Erysimum & 16 \\
Orobanchaceae & 20 & Epipactis & 15 \\
Violaceae & 20 & Pilosella & 15
\end{tabular}

et al. 2016a, 2016b, 2017a, 2017b), and on floristic contributions and taxonomic reviews.

For Italian endemic taxa, we referred to the recent inventory proposed by Peruzzi et al. (2014a), which is continuously updated (see http://goo.gl/x8QL4J). According to these authors, the "Italian endemic" status was attributed to those taxa occurring only in Italy, or in Italy and Corsica (France) or in Italy and Malta. 
Table 3. Most represented genera for each category of distribution data: $P(\geq 60$ taxa), NC $(\geq 2), E X(\geq 1), D(\geq 2)$ and DD ( $\geq 1)$.

\begin{tabular}{|c|c|c|c|c|c|c|c|c|c|}
\hline Genera & $P$ & Genera & $\mathrm{NC}$ & Genera & EX & Genera & $\mathrm{D}$ & Genera & DD \\
\hline Hieracium & 686 & Hieracium & 460 & Ranunculus & 5 & Hieracium & 21 & Taraxacum & 5 \\
\hline Taraxacum & 135 & Taraxacum & 12 & Limonium & 3 & Polygala & 3 & Cotoneaster & 2 \\
\hline Carex & 122 & Pilosella & 10 & Anthyllis & 2 & Alchemilla & 2 & Arenaria & 1 \\
\hline Centaurea & 115 & Alchemilla & 6 & Aldrovanda & 1 & Alyssum & 2 & Bromopsis & 1 \\
\hline Limonium & 108 & Atriplex & 3 & Bromus & 1 & Carex & 2 & Camelina & 1 \\
\hline Ranunculus & 103 & Carduus & 3 & Bulbostylis & 1 & Juncus & 2 & Crataegus & 1 \\
\hline Ophrys & 101 & Carex & 2 & Caldesia & 1 & Malva & 2 & Deschampsia & 1 \\
\hline Pilosella & 90 & Dianthus & 2 & Camelina & 1 & Ophrys & 2 & Dryopteris & 1 \\
\hline Alchemilla & 87 & Isolepis & 2 & Carlina & 1 & Paeonia & 2 & Helianthemum & 1 \\
\hline Silene & 84 & Lythrum & 2 & Castroviejoa & 1 & Potentilla & 2 & Noccaea & 1 \\
\hline Festuca & 82 & Onosma & 2 & Clematis & 1 & Saxifraga & 2 & Persicaria & 1 \\
\hline Trifolium & 78 & Polycnemum & 2 & Helianthemum & 1 & Spergularia & 2 & Phlomis & 1 \\
\hline Allium & 68 & Silene & 2 & Helosciadium & 1 & Taraxacum & 2 & Scorpiurus & 1 \\
\hline Saxifraga & 64 & & & Herniaria & 1 & Trifolium & 2 & Sesamoides & 1 \\
\hline \multirow[t]{5}{*}{ Euphorbia } & 62 & & & Launaea & 1 & & & & \\
\hline & & & & Puccinellia & 1 & & & & \\
\hline & & & & Sonchus & 1 & & & & \\
\hline & & & & Stratiotes & 1 & & & & \\
\hline & & & & Suaeda & 1 & & & & \\
\hline
\end{tabular}

The distribution data (see Appendix S2 in supplementary material for detailed information about the regional distribution) given for each of the 20 administrative regions of Italy (not considering the two enclave-countries Republic of San Marino and Vatican (ity State) are coded as follows: Valle d'Aosta, VDA; Piemonte, PIE; Lombardia, LOM; Trentino-Alto Adige, TAA; Veneto, VEN; Friuli Venezia Giulia, FVG; Liguria, LIG; Emilia-Romagna, EMR; Toscana, TOS; Marche, MAR; Umbria, UMB; Lazio, LAZ; Abruzzo, ABR; Molise, MOL; Puglia, PUG; Campania, CAM; Basilicata, BAS; Calabria, CAL; Sicilia, SIC; Sardegna, SAR. In the Appendix S2, when the information concerning the distribution of a given subspecies for a region is missing, only the occurrence at species level was reported.

For each region, the occurrence status of each taxon is provided using the following categories:

- Occurring: "P";

- Doubtfully occurring: "D";

- No longer recorded (reliable historical record): "NC";

- Extinct or possibly extinct: "EX";

- Recorded by mistake: "NP";

- Alien at regional level: "A" ["CAS" (Casual), "NAT" (Naturalized), "INV" (Invasive)].

- Other abbreviations or symbols used in the list before the species/subspecies name are as follows:

- Italian endemic: "E";

- Cryptogenic: " $C$ ", i.e. a doubtfully native taxon, whose origin of occurrence in Italy is unknown;

- Taxonomically doubtful: "T";

- Data deficient at national level: "DD".

The families in the checklist are systematically ordered and, within them, the genera, species and subspecies are alphabetically listed. The taxa doubtfully occurring in Italy are indicated in italics only (not in bold italics). Taxonomic references concerning genera are added below the families. Notes on taxonomy, nomenclature and distribution are included in the list after each taxon. The lists of synonyms, misapplied and included names (Appendix S3), along with the list of taxa to be excluded at national level (Appendix S4), are available as supplementary material.
Table 4. Number of taxa occurring in each of the 20 administrative regions.

\begin{tabular}{|c|c|c|c|}
\hline & $(\mathrm{N}+\mathrm{C})$ & $\begin{array}{c}\text { No. of taxa } \\
\mathrm{A}(\mathrm{N}+\mathrm{C}+\mathrm{A} \\
[\mathrm{NAT}, \mathrm{INV}])\end{array}$ & $\begin{array}{c}\mathrm{T}(\mathrm{N}+\mathrm{C}+\mathrm{A} \\
[\mathrm{CAS}, \mathrm{NAT}, \mathrm{INV}])\end{array}$ \\
\hline PIE & 3464 & 3508 & 3535 \\
\hline TOS & 3370 & 3391 & 3400 \\
\hline LOM & 3272 & 3303 & 3429 \\
\hline ABR & 3190 & 3207 & 3216 \\
\hline VEN & 3169 & 3268 & 3338 \\
\hline TAA & 3116 & 3238 & 3504 \\
\hline LAZ & 3003 & 3028 & 3047 \\
\hline LIG & 3002 & 3050 & 3080 \\
\hline FVG & 2975 & 3134 & 3147 \\
\hline CAM & 2813 & 2826 & 2828 \\
\hline EMR & 2798 & 2827 & 2843 \\
\hline CAL & 2768 & 2788 & 2799 \\
\hline SIC & 2763 & 2782 & 2787 \\
\hline BAS & 2598 & 2606 & 2607 \\
\hline PUG & 2552 & 2568 & 2577 \\
\hline MAR & 2497 & 2535 & 2540 \\
\hline UMB & 2364 & 2400 & 2406 \\
\hline MOL & 2305 & 2324 & 2327 \\
\hline SAR & 2301 & 2378 & 2441 \\
\hline VDA & 2289 & 2322 & 2333 \\
\hline
\end{tabular}

Notes: No. of taxa (native " $\mathrm{N}$ " + cryptogenic " $\mathrm{C}$ "), No. of taxa A (native " $\mathrm{N}$ " + cryptogenic " $C$ " + regionally non-native "A [NAT, INV]"), No. of taxa T (native " $N$ " + cryptogenic " $C$ " + regionally non-native "A [CAS, NAT, INV]"). Valle d'Aosta, VDA; Piemonte, PIE; Lombardia, LOM; Trentino-Alto Adige, TAA; Veneto, VEN; Friuli Venezia Giulia, FVG; Liguria, LIG; Emilia-Romagna, EMR; Toscana, TOS; Marche, MAR; Umbria, UMB; Lazio, LAZ; Abruzzo, ABR; Molise, MOL; Puglia, PUG; Campania, CAM; Basilicata, BAS; Calabria, CAL; Sicilia, SIC; Sardegna, SAR. CAS: Casual, NAT: Naturalized, INV: Invasive.

\section{Results}

The checklist includes 8195 taxa (6417 species and 1778 subspecies), belonging to 1092 genera and 152 families (Table 1). The lycophytes are represented by 3 families, 6 genera, 23 taxa and ferns and fern allies by 17 families, 34 genera and 108 taxa. The gymnosperms are represented by 4 families, 7 genera and 30 taxa. The angiosperms consist of 8034 taxa grouped in $128 \mathrm{fam}$ ilies and 1045 genera. The most represented families (>300 taxa) and genera (>100 taxa) are, respectively: Asteraceae, Poaceae, Fabaceae, Caryophyllaceae, Brassicaceae and Rosaceae; 


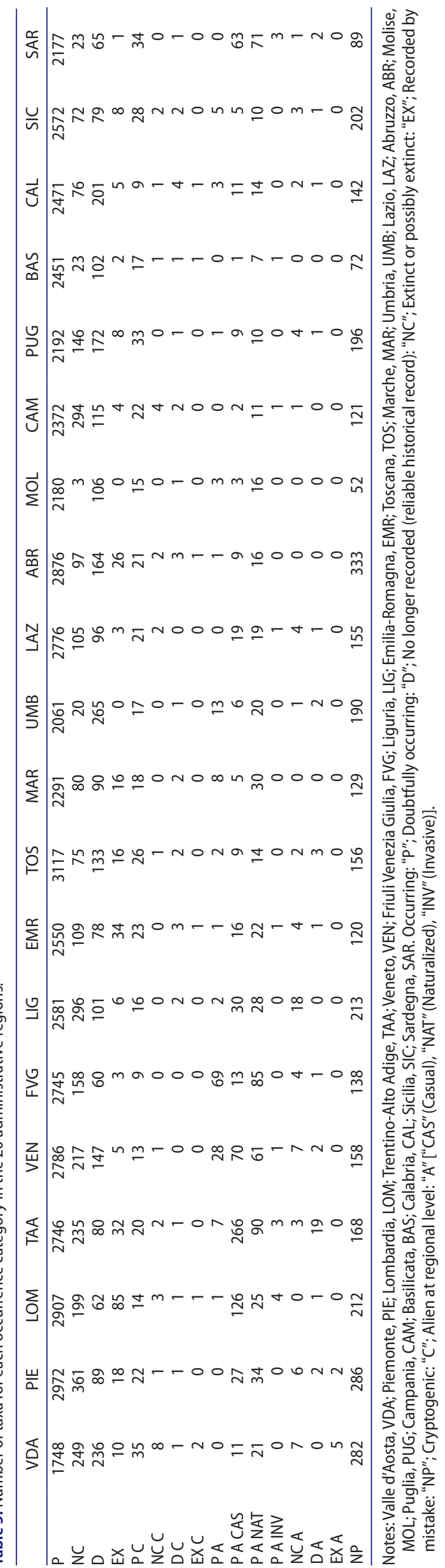

Table 6. Genera with the most represented number of cryptogenic and taxonomically doubtful taxa: C ( $\geq 2$ taxa); $T$ ( $\geq 5$ taxa).

\begin{tabular}{llll}
\hline Genera & C & \multicolumn{1}{c}{ Genera } & T \\
\hline Papaver & 4 & Ophrys & 74 \\
Portulaca & 4 & Stipa & 16 \\
Triticum & 4 & Anthyllis & 15 \\
Cuscuta & 3 & Centaurea & 15 \\
Digitaria & 2 & Carduus & 10 \\
Gladiolus & 2 & Polygala & 10 \\
Lycium & 2 & Dianthus & 8 \\
Rumex & 2 & Daucus & 7 \\
& & Sesleria & 6 \\
& & Viola & 6 \\
& & Anthemis & 5 \\
& & Crepis & 5 \\
& & Dactylis & 5 \\
& & Helianthemum & 5 \\
& & Helleborus & 5 \\
& & Micromeria & 5 \\
& & Rosa & 5 \\
& & Stachys & 5 \\
\hline
\end{tabular}

Hieracium, Taraxacum, Carex, Centaurea, Limonium, Ranunculus, Ophrys and Pilosella.

The taxa endemic to Italy are 1708 , grouped in 70 families and 313 genera. Four genera are narrowly endemic to Italy: Eokochia (Chenopodiaceae), Rhizobotrya (Brassicaceae), Petagnaea and Siculosciadium (Apiaceae). Three genera are endemic to Sardegna and Corse (France): Morisia (Brassicaceae), Castroviejoa and Nananthea (Asteraceae).

The most represented families ( $>70$ taxa) and genera $(>30$ taxa) concerning Italian endemic taxa are (Table 2): Asteraceae (582), Plumbaginaceae (117), Caryophyllaceae (97), Orchidaceae (87), Fabaceae (80), and Brassicaceae (78); Hieracium (337), Limonium (98), Centaurea (73), Ophrys (61), Ranunculus (37) and Taraxacum (33).

The taxa currently occurring in Italy are 7483 , while 568 taxa have not been confirmed in recent times, 99 are doubtfully occurring in the country and 19 are data deficient (Table 3 ). Out of the 568 not confirmed taxa, 26 are considered extinct or possibly extinct: Aldrovanda vesiculosa, Anthyllis hermanniae subsp. corsica, A. hermanniae subsp. sicula, Bromus grossus, Bulbostylis cioniana, Caldesia parnassifolia, Camelina alyssum subsp. alyssum, Carlina acanthifolia subsp. utzka, Castroviejoa frigida, Clematis integrifolia, Helianthemum syriacum subsp. thibaudii, Helosciadium repens, Herniaria fontanesii subsp. empedocleana, Launaea nudicaulis, Limonium catanense, $L$. intermedium, L. peucetium, Puccinellia gussonei, Ranunculus fiorii, R. fraelensis, R. hostiliensis, R. monspeliacus subsp. saxatilis, R. mutinensis, Sonchus palustris, Stratiotes aloides and Suaeda kocheri. The taxa to be excluded at national level are 177 (see Appendix S4).

The administrative regions showing the highest number of taxa (Tables 4 and 5), also considering the regionally non-native (CAS, NAT, INV) taxa, are: PIE (3535), TAA (3504), LOM (3429), TOS (3400), VEN (3338) and ABR (3216). By excluding the taxa regionally non-native (CAS, NAT, INV) at regional level, the regions with the highest number of taxa are: PIE (3464), TOS (3370), LOM (3272), ABR (3190), VEN (3169) and TAA (3116). Cryptogenic taxa are 56, while 430 taxa are taxonomically doubtful (Table 6). 


\section{Checklist of the Italian native vascular flora}

Lycophytes

Lycopodiaceae

Diphasiastrum alpinum (L.) Holub

Diphasiastrum complanatum (L.) Holub

Diphasiastrum issleri (Rouy) Holub

Diphasiastrum oellgaardii Stoor, Boudrie, Jérôme, Horn \& Bennert

Diphasiastrum tristachyum (Pursh) Holub

Diphasiastrum zeilleri (Rouy) Holub

Huperzia selago (L.) Bernh. ex Schrank \& Mart. subsp. selago

Lycopodiella inundata (L.) Holub

Lycopodium annotinum L. subsp. annotinum

Lycopodium clavatum L. - Note: Lycopodium lagopus (Laest. ex Hartm.) Zinserl. ex Kuzen. is a reduced form of. L. clavatum, without taxonomic value.

Isoëtaceae

Taxonomic references: Isoëtes (Troìa and Greuter 2014; Bagella et al. 2015).

Isoëtes durieui Bory

Isoëtes echinospora Durieu

Isoëtes gymnocarpa (Gennari) A.Braun

Isoëtes histrix Bory

Isoëtes longissima Bory

E Isoëtes malinverniana Ces. \& De Not.

E Isoëtes sabatina Troì \& Azella

Isoëtes sicula Tod.

Isoëtes tiguliana Gennari

Isoëtes todaroana Trò̀ \& Raimondo

Selaginellaceae

Selaginella denticulata (L.) Spring

Selaginella helvetica (L.) Spring

Selaginella selaginoides (L.) P.Beauv. ex Schrank \& Mart.

Ferns and fern allies

Equisetaceae

Equisetum arvense $\mathrm{L}$.

Equisetum fluviatile $\mathrm{L}$.

Equisetum hyemale L.

Equisetum palustre L.

Equisetum pratense Ehrh.

Equisetum ramosissimum Desf.

Equisetum sylvaticum $L$.

Equisetum telmateia Ehrh.

Equisetum variegatum Schleich. ex F.Weber \& D.Mohr

Ophioglossaceae

Botrychium lanceolatum (S.G.Gmel.) Ångstr.

Botrychium Iunaria (L.) SW.

Botrychium matricariifolium (A.Braun ex Döll) W.D.J.Koch

Botrychium simplex E. Hitchc.

Botrypus virginianus (L.) Michx.

Ophioglossum azoricum C.Presl - Note: The taxonomic identity of the population from VEN is highly questionable (Peruzzi et al. 2015c). According to the same authors, three hybrid taxa, morphologically similar to 0 . azoricum, occur in Italy.

Ophioglossum lusitanicum L.

Ophioglossum vulgatum L.

Sceptridium multifidum (S.G.Gmel.) M.Nishida ex Tagawa

Osmundaceae

Osmunda regalis L.

Hymenophyllaceae

Hymenophyllum tunbrigense (L.) Sm.

Vandenboschia speciosa (Willd.) G.Kunkel - Note: In LIG and in Monte Pisano (TOS), only gametophytes can be found.

Marsileaceae

Marsilea quadrifolia L.

Marsilea strigosa Willd.

Pilularia globulifera L.

Pilularia minuta Durieu ex A.Braun

Salviniaceae

Salvinia natans (L.) All.

Dennstaedtiaceae

Pteridium aquilinum (L.) Kuhn subsp. aquilinum 
Pteridaceae

Adiantum capillus-veneris $\mathrm{L}$.

Allosorus acrosticus (Balb.) Christenh.

Allosorus guanchicus (Bolle) Christenh.

Allosorus persicus (Bory) Christenh.

Allosorus pteridioides (Reichard) Christenh.

Allosorus tinaei (Tod.) Christenh.

Anogramma leptophylla (L.) Link

Cosentinia vellea (Aiton) Tod. subsp. bivalens (Reichst.) Rivas Mart. \& Salvo

Cosentinia vellea (Aiton) Tod. subsp. vellea

Cryptogramma crispa (L.) R.Br. ex Hook.

Paragymnopteris marantae (L.) K.H.Shing subsp. marantae

Pteris cretica L.

Pteris vittata $\mathrm{L}$

Cystopteridaceae

Cystopteris alpina (Lam.) Desv.

Cystopteris diaphana (Bory) Blasdell

Cystopteris dickieana R.Sim

Cystopteris fragilis (L.) Bernh.

Cystopteris montana (Lam.) Bernh. ex Desv.

Cystopteris sudetica A.Braun \& Milde

Gymnocarpium dryopteris (L.) Newman

Gymnocarpium robertianum (Hoffm.) Newman

Aspleniaceae

Asplenium adiantum-nigrum L. subsp. adiantum-nigrum

Asplenium adulterinum Milde subsp. adulterinum

Asplenium adulterinum Milde subsp. presolanense Mokry, Rasbach \& Reichst.

Asplenium balearicum Shivas

Asplenium ceterach L. subsp. bivalens (D.E.Mey.) Greuter \& Burdet

Asplenium ceterach L. subsp. ceterach

Asplenium ceterach L. subsp. cyprium (Viane \& Van den heede) Viane

Asplenium cuneifolium Viv. subsp. cuneifolium

Asplenium fissum Kit. ex Willd.

Asplenium fontanum (L.) Bernh. subsp. fontanum

Asplenium foreziense Le Grand ex Magnier - Note: For the nomenclature of this species, see Boudrie and Viane (2012).

Asplenium lepidum C.Presl subsp. lepidum

Asplenium marinum $\mathrm{L}$.

Asplenium obovatum Viv. subsp. billotii (F.W.Schultz ) O.Bolòs, Vigo, Masalles \& Ninot

Asplenium obovatum Viv. subsp. obovatum

Asplenium onopteris L.

Asplenium petrarchae (Guérin) DC. subsp. petrarchae

Asplenium ruta-muraria L. subsp. dolomiticum Lovis \& Reichst.

Asplenium ruta-muraria L. subsp. ruta-muraria

Asplenium sagittatum (DC.) Bange

Asplenium scolopendrium L. subsp. scolopendrium

Asplenium seelosii Leyb. subsp. seelosii

Asplenium septentrionale (L.) Hoffm. subsp. septentrionale

Asplenium trichomanes L. subsp. inexpectans Lovis - Note: The records from BAS, SIC and SAR refer to A. microphyllum Tineo, which is provisionally included here.

Asplenium trichomanes L. subsp. quadrivalens D.E.Mey.

Asplenium trichomanes L. subsp. pachyrachis (Christ) Lovis \& Reichst.

Asplenium trichomanes L. subsp. trichomanes

Asplenium viride Huds.

Thelypteridaceae

Oreopteris limbosperma (All.) Holub

Phegopteris connectilis (Michx.) Watt

Thelypteris palustris Schott

Woodsiaceae

Woodsia alpina (Bolton) Gray

Woodsia ilvensis (L.) R.Br.

Woodsia pulchella Bertol.

Onocleaceae

Matteuccia struthiopteris (L.) Tod.

Blechnaceae

Struthiopteris spicant (L.) Weiss

Woodwardia radicans (L.) Sm.

Athyriaceae

Athyrium filix-femina (L.) Roth

Pseudathyrium alpestre (Hoppe) Newman - Note: For the nomenclature of this species, see Wei et al. (2018). 
Dryopteridaceae

Taxonomic references: Dryopteris affinis (Lowe) Fraser-Jenk. group (Fraser-Jenkins 2007).

Dryopteris affinis (Lowe) Fraser-Jenk. subsp. affinis

Dryopteris borreri (Newman) Newman ex Oberh. \& Tavel.

DD Dryopteris cambrensis (Fraser-Jenk.) J.Beitel \& W.R.Buck subsp. distans (Viv.) Fraser-Jenk. - Note: Recorded for Italy by Fraser-Jenkins (2007), but without precise localities.

Dryopteris cambrensis (Fraser-Jenk.) J.Beitel \& W.R.Buck subsp. insubrica (Oberh. \& Tavel ex Fraser-Jenk.) Fraser-Jenk.

Dryopteris carthusiana (Vill.) H.P.Fuchs

Dryopteris dilatata (Hoffm.) A.Gray

Dryopteris expansa (C.Presl) Fraser-Jenk. \& Jermy

Dryopteris filix-mas (L.) Schott

Dryopteris mindshelkensis Pavlov

Dryopteris oreades Fomin

Dryopteris pallida (Bory) Maire \& Petitm. subsp. pallida

Dryopteris remota (A.Braun ex Döll) Druce

Dryopteris tyrrhena Fraser-Jenk. \& Reichst.

Dryopteris villarii (Bellardi) Woyn. ex Schinz \& Thell.

Polystichum aculeatum (L.) Roth

Polystichum braunii (Spenn.) Fée

Polystichum lonchitis (L.) Roth

Polystichum setiferum (Forssk.) T.Moore ex Woyn.

Polypodiaceae

Polypodium cambricum $\mathrm{L}$.

Polypodium interjectum Shivas

Polypodium vulgare L.

Gymnosperms

Ephedraceae

Ephedra distachya L. subsp. distachya

Ephedra distachya L. subsp. helvetica (C.A.Mey.) Asch. \& Graebn.

Ephedra foeminea Forssk.

Ephedra fragilis Desf.

Ephedra major Host subsp. major

Ephedra podostylax Boiss.

Pinaceae

Abies alba Mill.

E Abies nebrodensis (Lojac.) Mattei

Larix decidua Mill.

Picea abies (L.) H.Karst.

Picea obovata Ledeb.

Pinus cembra $\mathrm{L}$.

Pinus halepensis Mill. subsp. brutia (Ten.) Holmboe

Pinus halepensis Mill. subsp. halepensis

Pinus heldreichii Christ subsp. leucodermis (Antoine) E.Murray

Pinus mugo Turra subsp. mugo - Note: The records of Pinus rotundata Link [Pinus mugo Turra subsp. rotundata (Link) Janch. \& H.Neumayer] from Italy actually refer to Pinus ×ascendens Businský nothosubsp. ascendens (Businský and Kirschner 2006, 2010).

Pinus mugo Turra subsp. uncinata (Ramond ex DC.) Domin

Pinus nigra J.F.Arnold subsp. Iaricio Palib. ex Maire

Pinus nigra J.F.Arnold subsp. nigra

Pinus pinaster Aiton subsp. pinaster

Pinus sylvestris $\mathrm{L}$.

Cupressaceae

Taxonomic references: Juniperus phoenicea L. group (Mazur et al. 2016).

Juniperus communis L.

Juniperus deltoides R.P.Adams - Note: For a detailed study about the systematic relationships and the geographic distribution of this species in relation with $J$. macrocarpa and J. oxycedrus, see Roma-Marzio et al. (2017).

T Juniperus macrocarpa $\mathrm{Sm}$.

Juniperus oxycedrus $\mathrm{L}$.

Juniperus phoenicea $\mathrm{L}$.

Juniperus sabina $\mathrm{L}$.

Juniperus thurifera $\mathrm{L}$.

Juniperus turbinata Guss.

Taxaceae

Taxus baccata $\mathrm{L}$.

Angiosperms

Nymphaeaceae

Nuphar lutea (L.) Sm.

Nymphaea alba L. 
Lauraceae

Laurus nobilis L.

Aristolochiaceae

Aristolochia clematitis L.

E Aristolochia clusii Lojac.

Aristolochia lutea Desf.

Aristolochia navicularis E.Nardi

Aristolochia pallida Willd.

Aristolochia rotunda L. subsp. insularis (E.Nardi \& Arrigoni) Gamisans

Aristolochia rotunda $\mathrm{L}$. subsp. rotunda

Aristolochia sempervirens $L$.

E Aristolochia sicula Tineo

E Aristolochia tyrrhena E.Nardi \& Arrigoni

Asarum europaeum L.

Araceae

Ambrosina bassii L.

Arisarum proboscideum (L.) Savi

E Arisarum vulgare O.Targ.Tozz. subsp

Arum cylindraceum Gasp. ex Guss.

Arum italicum Mill. subsp. italicum

Arum maculatum L.

Arum pictum L.f. subsp. pictum

Biarum dispar (Schott) Talavera

Biarum tenuifolium (L.) Schott subsp. abbreviatum (Schott) K.Richt.

Biarum tenuifolium (L.) Schott subsp. tenuifolium

Dracunculus vulgaris Schott

Helicodiceros muscivorus (L.f.) Engl.

Lemna gibba L.

Lemna minor $\mathrm{L}$.

Lemna trisulca $\mathrm{L}$.

Spirodela polyrhiza (L.) Schleid.

Wolffia arrhiza (L.) Horkel ex Wimm.

Tofieldiaceae

Tofieldia calyculata (L.) Wahlenb.

Tofieldia pusilla (Michx.) Pers.

Alismataceae

Alisma gramineum Lej. subsp. gramineum

Alisma lanceolatum With.

Alisma plantago-aquatica $\mathrm{L}$.

Baldellia ranunculoides (L.) Parl.

Caldesia parnassifolia (Bassi ex L.) Parl. - Note: Possibly extinct in Italy (Rossi et al. 2013).

Damasonium alisma Mill. subsp. alisma

Damasonium alisma Mill. subsp. bourgaei (Coss.) Maire

Damasonium polyspermum Coss.

Sagittaria sagittifolia L.

Butomaceae

Butomus umbellatus $\mathrm{L}$.

Hydrocharitaceae

Hydrocharis morsus-ranae L.

Najas major All.

T Najas marina L. subsp. armata (H.Lindb.) Horn

Najas minor All.

Stratiotes aloides L. - Note: Possibly extinct in the wild (Rossi et al. 2013). Recently, this species was recorded as alien in VEN (Masin and Tietto 2006). Vallisneria spiralis L.

Scheuchzeriaceae

Scheuchzeria palustris $\mathrm{L}$.

Juncaginaceae

Triglochin barrelieri Loisel.

Triglochin Iaxiflora Guss.

Triglochin maritima $\mathrm{L}$

Triglochin palustris $\mathrm{L}$.

Zosteraceae

Taxonomic references: Tomlinson and Posluzny (2001), Kato et al. (2003).

Nanozostera noltei (Hornem.) Toml. \& Posl.

Zostera marina $\mathrm{L}$. 
Cymodoceaceae

Cymodocea nodosa (Ucria) Asch.

Potamogetonaceae

Taxonomic references: Zannichellia L. (Talavera et al. 1986).

Althenia filiformis Petit subsp. filiformis

Groenlandia densa (L.) Fourr.

Posidonia oceanica (L.) Delile

Potamogeton acutifolius Link

Potamogeton alpinus Balb.

Potamogeton berchtoldii Fieber

Potamogeton coloratus Hornem.

Potamogeton compressus $\mathrm{L}$.

Potamogeton crispus L.

Potamogeton friesii Rupr.

Potamogeton gramineus $\mathrm{L}$.

Potamogeton lucens L.

Potamogeton natans $\mathrm{L}$.

Potamogeton nodosus Poir.

Potamogeton obtusifolius Mert. \& W.D.J.Koch

Potamogeton perfoliatus $\mathrm{L}$.

Potamogeton polygonifolius Pourr.

Potamogeton praelongus Wulfen

Potamogeton pusillus L.

Potamogeton schweinfurthii A.Benn.

Potamogeton trichoides Cham. \& Schltdl.

Stuckenia filiformis (Pers.) Börner

Stuckenia pectinata (L.) Börner

Zannichellia obtusifolia Talavera, García-Mur. \& H.Smit

Zannichellia palustris L.

Zannichellia pedunculata Rchb.

Zannichellia peltata Bertol.

Ruppiaceae

Ruppia maritima L.

Ruppia spiralis L. ex Dumort.

Dioscoreaceae

Dioscorea communis (L.) Caddick \& Wilkin

Colchicaceae

Taxonomic references: Colchicum L. (Persson 2007, 2008; Fridlender 2009, 2016).

Colchicum alpinum DC.

Colchicum arenasii Fridl.

Colchicum autumnale L.

Colchicum bivonae Guss.

Colchicum bulbocodium Ker Gawl. subsp. bulbocodium

Colchicum bulbocodium Ker Gawl. subsp. versicolor (Ker Gawl.) K.Perss.

Colchicum cupanii Guss. subsp. cupanii

Colchicum gonarei Camarda

Colchicum longifolium Castagne

Colchicum Iusitanum Brot.

E Colchicum nanum K.Perss.

ET Colchicum neapolitanum (Ten.) Ten. subsp. gracile (K.Perss.) Fridl.

E Colchicum neapolitanum (Ten.) Ten. subsp. neapolitanum

Colchicum triphyllum Kunze

Colchicum verlaqueae Fridl.

Melanthiaceae

Paris quadrifolia L.

Veratrum album L.

Veratrum nigrum $\mathrm{L}$.

Smilacaceae

Smilax aspera L.

Liliaceae

Taxonomic references: Tulipa L. (Christenhusz et al. 2013); Gagea Salisb. (Peruzzi et al. 2008b, 2008c; Peruzzi 2012; Tison et al. 2013).

Erythronium dens-canis L.

Fritillaria burnatii (Planch.) Backh. - Note: The records of F. tubaeformis subsp. tubaeformis from Italy (Conti et al. 2005) actually refer to this taxon (Bartolucci and Peruzzi 2012; Bovio 2014; Mucciarelli et al. 2016).

Fritillaria involucrata All.

Fritillaria messanensis Raf. subsp. gracilis (Ebel) Rix - Note: For the distribution of this subspecies, see Peruzzi et al. (2017).

Fritillaria messanensis Raf. subsp. messanensis

Fritillaria montana Hoppe ex W.D.J.Koch - Note: For the nomenclature, taxonomy and distribution of this taxon, see Bartolucci et al. (2009), Peruzzi and Bartolucci (2009).

Fritillaria tubaeformis Gren. \& Godr. subsp. moggridgei (Boiss. \& Reuter ex Planch.) Rix 
Gagea amblyopetala Boiss. \& Heldr. Gagea apulica Peruzzi \& J.-M.Tison

Gagea bohemica (Zauschn.) Schult. \& Schult.f. - Note: For the taxonomy of this species, see Peterson et al. (2010).

Gagea chrysantha Schult. \& Schult.f.

Gagea foliosa (C.Presl) Schult. \& Schult.f.

Gagea fragifera (Vill.) Ehr.Bayer \& G.López - Note: For the nomenclature of this species, see Tison (2001).

Gagea granatellii (Parl.) Parl.

Gagea lacaitae A.Terracc.

Gagea lojaconoi Peruzzi

Gagea luberonensis J.-M.Tison

Gagea lutea (L.) Ker Gawl.

Gagea minima (L.) Ker Gawl.

Gagea peduncularis (J.Presl \& C.Presl) Pascher

Gagea peruzzii J.-M.Tison

Gagea polidorii J.-M.Tison

Gagea pratensis (Pers.) Dumort.

Gagea pusilla (F.W.Schmidt) Sweet

Gagea ramulosa A.Terracc.

Gagea serotina (L.) Ker Gawl.

Gagea sicula Lojac.

Gagea soleirolii F.W.Schultz ex Mutel

Gagea spathacea (Hayne) Salisb.

E Gagea tisoniana Peruzzi, Bartolucci, Frignani \& Minut.

Gagea trinervia (Viv.) Greuter

Gagea villosa (M.Bieb.) Sweet

Lilium bulbiferum L. subsp. bulbiferum

T Lilium bulbiferum L. subsp. croceum (Chaix) Jan

Lilium carniolicum Bernh. ex W.D.J.Koch

Lilium martagon $\mathrm{L}$.

Lilium pomponium $\mathrm{L}$.

Streptopus amplexifolius (L.) DC.

Tulipa pumila Moench

Tulipa sylvestris $\mathrm{L}$.

Orchidaceae

Taxonomic references: Ophrys L. (Romolini and Souche 2012; GIROS 2016); Coeloglossum Hartm. (Devos et al. 2006).

Anacamptis collina (Banks \& Sol. ex Russell) R.M.Bateman, Pridgeon \& M.W.Chase

Anacamptis coriophora (L.) R.M.Bateman, Pridgeon \& M.W.Chase

Anacamptis laxiflora (Lam.) R.M.Bateman, Pridgeon \& M.W.Chase

Anacamptis longicornu (Poir.) R.M.Bateman, Pridgeon \& M.W.Chase

Anacamptis morio (L.) R.M.Bateman, Pridgeon \& M.W.Chase - Note: The record of A. morio subsp. caucasica (K.Koch) H.Kretzschmar from PUG (Griebl 2010) is included here.

Anacamptis palustris (Jacq.) R.M.Bateman, Pridgeon \& M.W.Chase

Anacamptis papilionacea (L.) R.M.Bateman, Pridgeon \& M.W.Chase

Anacamptis pyramidalis (L.) Rich.

Barlia robertiana (Loisel.) Greuter

Cephalanthera damasonium (Mill.) Druce

Cephalanthera longifolia (L.) Fritsch

Cephalanthera rubra (L.) Rich.

Chamorchis alpina (L.) Rich.

Coeloglossum viride (L.) Hartm.

Corallorhiza trifida Châtel.

Cypripedium calceolus $\mathrm{L}$.

Dactylorhiza elata (Poir.) Soó subsp. sesquipedalis (Willd.) Soó

Dactylorhiza incarnata (L.) Soó subsp. cruenta (O.F.Müll.) P.D.Sell

Dactylorhiza incarnata (L.) Soó subsp. incarnata

Dactylorhiza insularis (Sommier) Ó.Sánchez \& Herrero

Dactylorhiza lapponica (Laest. ex Hartm.) Soó subsp. angustata (Arv.-Touv.) Kreutz

Dactylorhiza lapponica (Laest. ex Hartm.) Soó subsp. rhaetica H.Baumann \& R.Lorenz

Dactylorhiza maculata (L.) Soó subsp. fuchsii (Druce) Hyl.

Dactylorhiza maculata (L.) Soó subsp. saccifera (Brongn.) Diklić

Dactylorhiza maculata (L.) Soó subsp. savogiensis (D.Tyteca \& Gathoye) Kreutz

Dactylorhiza majalis (Rchb.) P.F.Hunt \& Summerh.

Dactylorhiza romana (Sebast.) Soó subsp. markusii (Tineo) Holub

Dactylorhiza romana (Sebast.) Soó subsp. romana

Dactylorhiza sambucina (L.) Soó

Dactylorhiza traunsteineri (Saut. ex Rchb.) Soó

Epipactis aspromontana Bartolo, Pulv. \& Robatsch

Epipactis atrorubens (Hoffm.) Besser

Epipactis autumnalis Doro

Epipactis bugacensis Robatsch

E Epipactis calabrica U.Grabner, S. Hertel \& Presser

E Epipactis collaris S.Hertel

E Epipactis cupaniana C.Brullo, D'Emerico \& Pulv.

Epipactis distans Art.-Touv.

E Epipactis etrusca Presser \& S. Hertel

Epipactis exilis P.Delforge 
Epipactis fageticola (C.E.Hermos.) Devillers-Tersch. \& Devillers

Epipactis greuteri H.Baumann \& Künkele

Epipactis helleborine (L.) Crantz

Epipactis hyblaea Brullo \& Zimmitti

ET Epipactis ioessa Bongiorni, De Vivo, Fori \& Romolini

Epipactis leptochila (Godfery) Godfery - Note: Epipactis savelliana Bongiorni, De Vivo \& Fori is provisionally included here.

Epipactis lucana Presser, S.Hertel \& V.A.Romano

Epipactis maricae (Croce, Bongiorni, De Vivo \& Fori) Presser \& S.Herte

Epipactis meridionalis H.Baumann \& R.Lorenz

Epipactis microphylla (Ehrh.) Sw.

Epipactis muelleri Godfery

Epipactis naousensis Robatsch

Epipactis neglecta (Kümpel) Kümpel

Epipactis palustris (L.) Crantz

Epipactis placentina Bongiorni \& Grünanger

Epipactis pontica Taubenheim

Epipactis purpurata Sm

Epipactis rhodanensis Gévaudan \& Robatsch

Epipactis rivularis Kranjčev \& Čičmir

Epipactis sanguinea S. Hertel \& Presser

Epipactis schubertiorum Bartolo, Pulv. \& Robatsch

Epipactis thesaurensis Agrezzi, Ovatoli \& Bongiorni

Epipactis zaupolensis (Barbaro \& Kreutz) Bongiorni, De Vivo \& Fori

Epipogium aphyllum Sw.

Gennaria diphylla (Link) Parl.

Goodyera repens (L.) R.Br.

Gymnadenia conopsea (L.) R.Br.

Gymnadenia densiflora (Wahlenb.) A.Dietr.

Gymnadenia odoratissima (L.) Rich.

Herminium monorchis (L.) R.Br.

Himantoglossum adriaticum H.Baumann

Himantoglossum hircinum (L.) Spreng.

Limodorum abortivum (L.) Sw.

Limodorum brulloi Bartolo \& Pulv.

Limodorum trabutianum Batt.

Liparis loeselii (L.) Rich.

Liparis nemoralis (Perazza, Decarli, Filippin, Bruna \& Regattin) Bartolucci \& Galasso - Note: This species was considered as endemic to Italy (Perazza et al. 2012

Perazza and Lorenz 2013; Peruzzi et al. 2014a; Bartolucci and Galasso 2016), but is has been recently recorded also for Austria by Stöhr (2016).

Malaxis monophyllos (L.) SW.

Malaxis paludosa (L.) Sw.

Neotinea lactea (Poir.) R.M.Bateman, Pridgeon \& M.W.Chase

Neotinea maculata (Desf.) Stearn

Neotinea tridentata (Scop.) R.M.Bateman, Pridgeon \& M.W.Chase

Neotinea ustulata (L.) R.M.Bateman, Pridgeon \& M.W.Chase

Neottia cordata (L.) Rich.

Neottia nidus-avis (L.) Rich.

Neottia ovata (L.) Bluff \& Fingerh.

Nigritella buschmanniae Teppner \& Ster

Nigritella corneliana (Beauverd) Gölz \& H.R.Reinhard

Nigritella lithopolitanica Ravink

Nigritella miniata (Crantz) Janch

Nigritella nigra (L.) Rchb. subsp. austriaca Teppner \& E.Klein - Note: This subspecies was discovered in 2017 in PIE by C. Lasen and A. Selvaggi (unpublished data).

Nigritella rhellicani Teppner \& E.Klein

Nigritella widderi Teppner \& E.Klein

Ophrys annae Devillers-Tersch. \& Devillers

Ophrys apifera Huds.

Ophrys appennina Romolini \& Soca

Ophrys apulica (O.Danesch \& E.Danesch) O.Danesch \& E.Danesch

Ophrys archimedea P.Delforge \& M.Walravens

Ophrys argentaria Devillers-Tersch. \& Devillers

Ophrys ausonia Devillers, Devillers-Tersch. \& P.Delforge

Ophrys bertolonii Moretti subsp. benacensis (Reisigl) P.Delforge

Ophrys bertolonii Moretti subsp. bertolonii

Ophrys bertolonii Moretti subsp. bertoloniiformis (O.Danesch \& E.Danesch) H.Sund

Ophrys bertolonii Moretti subsp. explanata (Lojac.) Soca

Ophrys bertolonii Moretti subsp. saratoi (E.G.Camus) Soca

Ophrys biancae (Tod.) Macch.

Ophrys bilunulata Risso

E Ophrys biscutella O.Danesch \& E.Danesch

Ophrys bombyliflora Link

Ophrys brutia P.Delforge

Ophrys caesiella P.Delforge

Ophrys calliantha Bartolo \& Pulv.

Ophrys calocaerina Devillers-Tersch. \& Devillers

Ophrys candica (E.Nelson ex Soó) H.Baumann \& Künkele

Ophrys chestermanii (J.J.Wood) Gölz \& H.R.Reinhard

Ophrys cinnabarina Romolini \& Soca 
Ophrys classica Devillers-Tersch. \& Devillers

Ophrys conradiae Melki \& Deschatres

Ophrys corsica Soleirol ex G.Foelsche \& W.Foelsche

Ophrys crabronifera Mauri

Ophrys delforgei Devillers-Tersch. \& Devillers

Ophrys dinarica Kranjčev \& P.Delforge

Ophrys exaltata Ten. subsp. arachnitiformis (Gren. \& M.Philippe) Del Prete

Ophrys exaltata Ten. subsp. archipelagi (Gölz \& H.R.Reinhard) Del Prete

Ophrys exaltata Ten. subsp. exaltata

Ophrys exaltata Ten. subsp. montis-leonis (O.Danesch \& E.Danesch) Soca

Ophrys exaltata Ten. subsp. morisii (Martelli) Del Prete

Ophrys exaltata Ten. subsp. splendida (Gölz \& H.R.Reinhard) Soca

Ophrys flammeola P.Delforge

Ophrys forestieri (Rchb.f.) Lojac.

Ophrys funerea Viv.

Ophrys gackiae P.Delforge

Ophrys gracilis (Büel, O.Danesch \& E.Danesch) Paulus

Ophrys gravinensis D'Alonzo

Ophrys gresivaudanica O.Gerbaud

Ophrys holosericea (Burnm.f.) Greuter subsp. holosericea

Ophrys holosericea (Burnm.f.) Greuter subsp. Iorenae (E.De Martino \& Centur.) Kreutz

Ophrys incubacea Bianca

Ophrys insectifera L.

Ophrys iricolor Desf. subsp. eleonorae (Devillers-Tersch. \& Devillers) Paulus \& Gack ex Kreutz

Ophrys lacaitae Lojac.

Ophrys Iaurensis Geniez \& Melki

Ophrys lepida S.Moingeon \& J.-M.Moingeon

Ophrys ligustica Romolini \& Soca

Ophrys litigiosa E.G.Camus

Ophrys Iojaconoi P.Delforge

Ophrys Iucana P.Delforge, Devillers-Tersch. \& Devillers

Ophrys Iucifera Devillers-Tersch. \& Devillers

Ophrys Iunulata Parl.

Ophrys lutea Cav.

Ophrys maritima Pacifico \& Soca

Ophrys marmorata G.Foelsche \&W.Foelsche

Ophrys massiliensis Viglione \& Véla

Ophrys mattinatae Medagli, A.Rossini, Quitadamo, D'Emerico \& Turco

Ophrys minipassionis Romolini \& Soca

Ophrys mirabilis Geniez \& Melki

Ophrys molisana P.Delforge

Ophrys murgiana Cillo, Medagli \& Margh.

Ophrys normanii J.J.Wood

Ophrys obaesa Lojac.

Ophrys oestrifera M.Bieb. subsp. montis-gargani Van de Vijver \& W.Looken

Ophrys ortuabis M.P.Grasso \& Manca

Ophrys oxyrrhynchos Tod. subsp. celiensis (O.Danesch \& E.Danesch) Del Prete

Ophrys oxyrrhynchos Tod. subsp. ingrassiae Dura, Turco, Gennaio \& Medagli

Ophrys oxyrrhynchos Tod. subsp. oxyrrhynchos

Ophrys pallida Raf.

Ophrys panattensis Scrugli, Cogoni \& Pessei

Ophrys panormitana (Tod.) Soó

Ophrys parvimaculata (O.Danesch \& E.Danesch) Paulus \& Gack

Ophrys passionis Sennen ex Devillers-Tersch. \& Devillers subsp. majellensis (Helga Daiss \& Herm.Daiss) Soca

Ophrys passionis Sennen ex Devillers-Tersch. \& Devillers subsp. passionis

Ophrys peucetiae Lozito, D'Emerico, Medagli \& Turco

Ophrys pinguis Romolini \& Soca

Ophrys pollinensis E.Nelson ex Devillers-Tersch. \& Devillers

Ophrys promontorii O.Danesch \& E.Danesch

Ophrys pseudoatrata S. Hertel \& Presser

Ophrys pseudomelena Turco, Medagli \& D'Emerico

Ophrys pseudoscolopax (Moggr.) Paulus \& Gack

Ophrys riojana C.E.Hermos.

Ophrys santonica J.M.Mathé \& Melki

Ophrys scolopax Cav. subsp. apiformis (Desf.) Maire \& Weiller

Ophrys scolopax Cav. subsp. cornuta (Steven) E.G.Camus

Ophrys sicula Tineo

Ophrys sipontensis (Gumpr.) O.Danesch \& E.Danesch

Ophrys speculum Link

Ophrys sphegodes Mill. subsp. grassoana Cristaudo, Galesi, R.Lorenz \& Zelesny

Ophrys sphegodes Mill. subsp. sphegodes

Ophrys tardans O.Danesch \& E.Danesch

Ophrys tarentina Gölz \& H.R.Reinhard

Ophrys tarquinia P.Delforge

Ophrys tenthredinifera Willd. subsp. aprilia (Devillers \& Devillers-Tersch.) Kreutz

Ophrys tenthredinifera Willd. subsp. grandiflora (Ten.) Kreutz

Ophrys tenthredinifera Willd. subsp. neglecta (Parl.) E.G.Camus

Ophrys tetraloniae W.P.Teschner 
Ophrys untchjii (M.Schulze) P.Delforge

Ophrys vetula Risso

Orchis anthropophora (L.) All.

Orchis brancifortii Biv.

Orchis italica Poir.

ET Orchis mascula (L.) L. subsp. ichnusae Corrias

Orchis mascula (L.) L. subsp. mascula

T Orchis mascula (L.) L. subsp. olbiensis (Reut. ex Gren.) Asch. \& Graebn.

T Orchis mascula (L.) L. subsp. speciosa (Mutel) Hegi

Orchis militaris L.

Orchis pallens $\mathrm{L}$.

Orchis patens Desf.

Orchis pauciflora Ten.

Orchis provincialis Balb. ex Lam. \& DC.

Orchis purpurea Huds.

Orchis quadripunctata Cirillo ex Ten.

Orchis simia Lam.

Orchis spitzelii Saut. ex W.D.J.Koch

Platanthera algeriensis Batt. \& Trab.

Platanthera bifolia (L.) Rich.

Platanthera chlorantha (Custer) Rchb.

Platanthera kuenkelei H.Baumann subsp. kuenkelei

Pseudorchis albida (L.) Á.Löve \& D.Löve

Serapias bergonii E.G.Camus

Serapias cordigera L. subsp. Iucana R.Lorenz \& V.A.Romano

Serapias cordigera L. subsp. cordigera

E Serapias cossyrensis B.Baumann \& H.Baumann

E Serapias francavillae Cristaudo, Galesi \& R.Lorenz

E Serapias intermedia Forest. ex F.W.Schultz subsp. hyblaea Cristaudo, Galesi \& R.Lorenz

Serapias lingua $\mathrm{L}$.

Serapias neglecta De Not.

Serapias nurrica Corrias subsp. nurrica

ET Serapias nurrica Corrias subsp. santuingensis (Senis, M.P.Grasso \& Orrù) Senis, M.P.Grasso \& Orrù

E Serapias orientalis (Greuter) H.Baumann \& Künkele subsp. apulica H.Baumann \& Künkele

E Serapias orientalis (Greuter) H.Baumann \& Künkele subsp. siciliensis Bartolo \& Pulv.

Serapias parviflora Parl.

Serapias politisii Renz

Serapias strictiflora Welw. ex Veiga subsp. gregaria (Godf.) Kreutz

Serapias vomeracea (Burm.f.) Briq.

Spiranthes aestivalis (Poir.) Rich.

Spiranthes spiralis (L.) Chevall.

Traunsteinera globosa (L.) Rchb.

Iridaceae

Taxonomic references: Chamaeiris Medik., Hermodactylus Mill., Iris L. s.str., Juno Tratt., Limniris (Tausch) Rchb., Moraea Mill., and Xiphion Mill. (Peruzzi et al.

2014b; Crespo et al. 2015); Crocus L. ser. Verni B.Mathew (Harpke et al. 2015).

Chamaeiris foetidissima (L.) Medik.

Chamaeiris graminea (L.) Medik.

Chamaeiris lorea (Janka) Peruzzi, F.Conti \& Bartolucci

E Crocus biflorus Mill. - Note: For the taxonomy and distribution of this species, see Harpke et al. (2016).

E Crocus etruscus Parl.

Crocus heuffelianus Herb. - Note: For the taxonomy and distribution of this species, see Peruzzi (2016).

E Crocus ilvensis Peruzzi \& Carta - Note: For the taxonomy and distribution of this species, see Peruzzi and Carta (2011).

E Crocus imperati Ten

Crocus ligusticus Mariotti

Crocus longiflorus Raf.

E Crocus minimus DC.

Crocus neapolitanus (Ker Gawl.) Loisel.

Crocus neglectus Peruzzi \& Carta

E Crocus siculus Tineo ex Guss.

E Crocus suaveolens Bertol.

Crocus thomasii Ten.

Crocus variegatus Hoppe \& Hornsch. - Note: For the taxonomy of this species, see Harpke et al. (2014).

Crocus vernus (L.) Hill - Note: For the typification of this name, see Peruzzi et al. (2013b).

Crocus versicolor Ker Gawl.

Crocus weldenii Hoppe \& Fürnr.

C Gladiolus byzantinus Mill.

Gladiolus dubius Guss.

Gladiolus illyricus W.D.J.Koch

Gladiolus imbricatus L.

C Gladiolus italicus Mill.

Gladiolus palustris Gaudin

Hermodactylus tuberosus (L.) Mill.

Iris benacensis A.Kern. ex Stapf

E Iris bicapitata Colas.

ET Iris calabra (N.Terracc.) Peruzzi

E Iris cengialti Ambrosi ex A.Kern. subsp. cengialti 
Romulea ramiflora Ten. subsp. ramiflor

Romulea requienii Parl.

E Romulea revelierei Jord. \& Fourr.

Romulea rollii Parl.

E Romulea variicolor Mifsud

Xiphion junceum (Poir.) Parl.

Xiphion vulgare Mill.

Asphodelaceae

Asphodeline liburnica (Scop.) Rchb.

Asphodeline lutea (L.) Rchb.

T Asphodelus albus Mill. subsp. delphinensis (Gren. \& Godr.) Z.Díaz \& Valdés

Asphodelus ayardii Jahand. \& Maire

Asphodelus cerasiferus J.Gay

Asphodelus fistulosus $\mathrm{L}$.

Asphodelus macrocarpus Parl. subsp. macrocarpus

Asphodelus ramosus L. subsp. ramosus

Asphodelus tenuifolius Cav.

Hemerocallis lilioasphodelus L.

Simethis mattiazzi (Vandelli) Saccardo

Amaryllidaceae

Taxonomic references: Sternbergia Waldst. \& Kit. (Peruzzi et al. 2008a).

Acis autumnalis (L.) Sweet

Acis nicaeensis (Ardoino) Lledó, A.P.Davis \& M.B.Crespo

E Acis rosea (F.Martin bis) Sweet

Allium acutiflorum Loisel.

E Allium aetnense Brullo, Pavone \& Salmeri

E Allium agrigentinum Brullo \& Pavone

Allium amethystinum Tausch

Allium angulosum $\mathrm{L}$.

E Allium anzalonei Brullo, Pavone \& Salmeri

E Allium apulum Brullo, Guglielmo, Pavone \& Salmeri

Allium atroviolaceum Boiss.

E Allium calabrum (N.Terracc.) Brullo, Pavone \& Salmeri

Allium carinatum $\mathrm{L}$.

E Allium castellanense (Garbari, Miceli \& Raimondo) Brullo, Guglielmo, Pavone \& Salmeri

Allium chamaemoly L. subsp. chamaemoly

Allium chamaespathum Boiss.

Allium coloratum Spreng.

Allium commutatum Guss.

E Allium cupanii Raf.

Allium cyrilli Ten. - Note: For the taxonomy and distribution of this species, see Peruzzi et al. (2012a).

E Allium diomedeum Brullo, Guglielmo, Pavone \& Salmeri

Allium ericetorum Thore

Allium flavum $\mathrm{L}$. subsp. flavum

Allium franciniae Brullo \& Pavone

Allium garbarii Peruzz

Allium garganicum Brullo, Pavone, Salmeri \& Terrasi

Allium hemisphaericum (Sommier) Brullo

Allium horvatii Lovrić

Allium insubricum Boiss. \& Reut. ex Reut.

Allium julianum Brullo, Gangale \& Uzunov

Allium lehmannii Lojac.

Allium longispathum Redouté - Note: There is a nomenclatural dispute concerning the application of the name A. longispathum Redouté. This name, if treated as conspecific with A. dentiferum, holds priority (a view supported by Jauzein and Tison 2001; which is followed here). On the contrary, Brullo et al. (2008) consider A. dentiferum as a taxon different from A. longispathum. The latter name, according to Brullo and Guarino (2017), should be applied to plants historically known in FVG, for Trieste area, and formerly called A. paniculatum L. The latter species, repeatedly wrongly recorded for Italy, is actually limited to E Europe (S Russia, Ukraine; Salmeri et al. 2016). 
Allium lopadusanum Bartolo, Brullo \& Pavone

Allium Iusitanicum Lam.

Allium moschatum $\mathrm{L}$.

Allium narcissiflorum Vill.

Allium neapolitanum Cirillo

ET Allium nebrodense Guss.

Allium nigrum $\mathrm{L}$.

E Allium obtusiflorum DC.

Allium ochroleucum Waldst. \& Kit.

Allium oleraceum $\mathrm{L}$. subsp. oleraceum

Allium oporinanthum Brullo, Pavone \& Salmeri

Allium pallens $\mathrm{L}$.

E Allium panormitanum Brullo, Pavone \& Salmeri

E Allium parciflorum Viv.

E Allium pelagicum Brullo, Pavone \& Salmeri

Allium pendulinum Ten.

E Allium pentadactyli Brullo, Pavone \& Spamp.

Allium permixtum Guss.

Allium polyanthum Schult. \& Schult.f.

Allium roseum $\mathrm{L}$. subsp. roseum

Allium rotundum $\mathrm{L}$.

E Allium samniticum Brullo, Pavone \& Salmeri

Allium sardoum Moris

Allium savii Parl.

Allium scaberrimum M.Serres

Allium schoenoprasum L. subsp. schoenoprasum

Allium siculum Ucria

Allium sphaerocephalon L. subsp. arvense (Guss.) Arcang.

ET Allium sphaerocephalon L. subsp. Iaxiflorum (Guss.) Giardina \& Raimondo

Allium sphaerocephalon L. subsp. sphaerocephalon

Allium strictum Schrad.

Allium suaveolens Jacq.

Allium subhirsutum $\mathrm{L}$. subsp. subhirsutum

Allium tenuiflorum Ten.

Allium trifoliatum Cirillo

Allium triquetrum $\mathrm{L}$.

Allium ursinum $\mathrm{L}$.

E Allium vernale Tineo

Allium victorialis $\mathrm{L}$.

Allium vineale $L$.

Galanthus nivalis $\mathrm{L}$.

Galanthus reginae-olgae Orph. subsp. reginae-olgae

T Galanthus reginae-olgae Orph. subsp. vernalis Kamari

Leucojum aestivum L. subsp. aestivum

Leucojum aestivum L. subsp. pulchellum (Salisb.) Briq.

Leucojum vernum $L$.

Narcissus miniatus Donn.-Morg., Koop. \& Zonn.

Narcissus obsoletus (Haw.) Steud.

Narcissus poëticus L.

Narcissus pseudonarcissus L. subsp. provincialis (Pugsley) J.-M.Tison - Note: The taxonomic identity of the populations from Italian Alps (LIG and PIE) is doubtful and needs further studies.

Narcissus pseudonarcissus L. subsp. pseudonarcissus - Note: Some populations from LIG and PIE are native.

E Narcissus supramontanus Arrigoni subsp. cunicularium Arrigoni

E Narcissus supramontanus Arrigoni subsp. supramontanus

Narcissus tazetta L. subsp. aureus (Loisel.) Baker

Narcissus tazetta L. subsp. italicus (Ker Gawl.) Baker

Narcissus tazetta L. subsp. tazetta

E Pancratium illyricum L. - Note: For the typification of this name, see Peruzzi et al. (2013c).

Pancratium maritimum $\mathrm{L}$.

Sternbergia colchiciflora Waldst. \& Kit.

Sternbergia lutea (L.) Ker Gawl. ex Spreng.

Sternbergia sicula Tineo ex Guss.

Asparagaceae

Anthericum liliago L.

Anthericum ramosum $\mathrm{L}$.

Aphyllanthes monspeliensis $\mathrm{L}$.

Asparagus acutifolius $\mathrm{L}$.

Asparagus albus L.

Asparagus aphyllus $\mathrm{L}$.

Asparagus horridus $\mathrm{L}$.

Asparagus maritimus (L.) Mill.

Asparagus officinalis $L$. subsp. officinalis

Asparagus pastorianus Webb \& Berthel.

Asparagus tenuifolius Lam.

Bellevalia boissieri Freyn - Note: For the taxonomy of this species with respect to B. dubia, see Borzatti von Loewenstern et al. (2013).

Bellevalia ciliata (Cirillo) T.Nees 
E Bellevalia dubia (Guss.) Rchb.

E Bellevalia pelagica C.Brullo, Brullo \& Pasta - Note: Unique Italian representative of a N African tetraploid group (Astuti et al. 2017a).

Bellevalia romana $(\mathrm{L}$.) Sweet

Bellevalia trifoliata (Ten.) Kunth

E Bellevalia webbiana Parl. - Note: For the biology and distribution of this species, see Astuti et al. (2018) and literature cited therein. Brimeura fastigiata (Viv.) Chouard

E Charybdis glaucophylla Bacch., Brullo, D'Emerico, Pontec. \& Salmeri

Charybdis maritima (L.) Speta

Charybdis numidica (Jord. \& Fourr.) Speta

Charybdis pancration (Steinh.) Speta

Charybdis undulata (Desf.) Speta

Chouardia litardierei (Breistr.) Speta

Convallaria majalis L.

Dipcadi serotinum (L.) Medik.

Hyacinthoides italica (L.) Rothm.

Loncomelos brevistylus (Wolfner) Dostál

Loncomelos narbonensis (L.) Raf.

Loncomelos pyrenaicus (L.) L.D.Hrouda subsp. pyrenaicus

T Loncomelos pyrenaicus (L.) L.D.Hrouda subsp. sphaerocarpus (A.Kern.) Holub

Maianthemum bifolium (L.) F.W.Schmidt

Melomphis arabica (L.) Raf.

Muscari botryoides (L.) Mill. subsp. botryoides

ET Muscari botryoides (L.) Mill. subsp. Iongifolium (Rigo) Garbari

Muscari commutatum Guss.

Muscari comosum (L.) Mill.

E Muscari gussonei (Parl.) Nyman

Muscari kerneri (Marches.) Soldano

Muscari neglectum Guss. ex Ten.

Muscari parviflorum Desf.

Muscari tenuiflorum Tausch

Oncostema ceruleum (Raf.) Speta

E Oncostema dimartinoi (Brullo \& Pavone) F.Conti \& Soldano

Oncostema elongatum (Parl.) Speta

E Oncostema siculum (Tineo) Speta

E Oncostema ughii (Tineo) Speta

Ornithogalum collinum Guss. subsp. collinum

Ornithogalum comosum $\mathrm{L}$.

E Ornithogalum corsicum Jord. \& Fourr.

Ornithogalum divergens Boreau

E Ornithogalum etruscum Parl. subsp. etruscum

ET Ornithogalum etruscum Parl. subsp. umbratile (Tornad. \& Garbari) Peruzzi \& Bartolucci

E Ornithogalum exscapum Ten.

Ornithogalum gussonei Ten.

Ornithogalum kochii Parl. subsp. kochii

Ornithogalum kochii Parl. subsp. monticola (Jord. \& Fourr.) Peruzzi

Ornithogalum montanum Cirillo ex Ten.

ET Ornithogalum orthophyllum Ten. - Note: Its systematic relationships with O. etruscum need further biosystematic studies. In addition, both names are yet to be typified.

Ornithogalum refractum Willd.

Ornithogalum umbellatum $\mathrm{L}$.

Paradisea liliastrum (L.) Bertol.

Polygonatum multiflorum (L.) All.

Polygonatum odoratum (Mill.) Druce

Polygonatum verticillatum (L.) All.

Prospero autumnale (L.) Speta

E Prospero corsicum (Boullu) J.-M.Tison

Prospero elisae Speta

E Prospero hierae Brullo, C.Brullo, Giusso, Pavone \& Salmeri

Prospero obtusifolium (Poir.) Speta subsp. intermedium (Guss.) Soldano \& F.Conti

Ruscus aculeatus $\mathrm{L}$.

Ruscus hypoglossum $\mathrm{L}$.

C Ruscus hypophyllum L.

Scilla bifolia L.

Urginea fugax (Moris) Steinh.

Arecaceae

Chamaerops humilis L.

Typhaceae

Sparganium angustifolium Michx.

Sparganium emersum Rehmann

Sparganium erectum $L$.

Sparganium hyperboreum Laest. ex Beurl.

Sparganium microcarpum (Neuman) Čelak.

Sparganium natans $\mathrm{L}$.

Sparganium neglectum Beeby

Typha angustifolia L.

Typha domingensis (Pers.) Steud. 
Typha latifolia L.

Typha laxmannii Lepech.

Typha minima Funk ex Hoppe

Typha shuttleworthii W.D.J.Koch \& Sond.

Juncaceae

Taxonomic references: Oreojuncus Záv.Drábk. \& Kirschner (Záveská Drábková and Kirschner 2013).

Juncus acutiflorus Ehrh. ex Hoffm.

Juncus acutus L. subsp. acutus

Juncus alpinoarticulatus Chaix subsp. alpinoarticulatus

Juncus anceps Laharpe

Juncus arcticus Willd.

Juncus articulatus L. subsp. articulatus

Juncus atratus Krock.

Juncus bufonius $\mathrm{L}$.

Juncus bulbosus $L$.

Juncus capitatus Weigel

Juncus castaneus Sm.

Juncus compressus Jacq.

Juncus conglomeratus $\mathrm{L}$.

Juncus effusus $L$. subsp. effusus

Juncus filiformis $\mathrm{L}$.

Juncus foliosus Desf.

Juncus fontanesii J.Gay subsp. fontanesii

Juncus fontanesii J.Gay subsp. pyramidatus (Laharpe) Snogerup

Juncus gerardi Loisel. subsp. gerardi

Juncus heterophyllus Dufour

Juncus hybridus Brot. - Note: According to Kirschner (2002), J. ambiguus Guss. is included here.

Juncus inflexus L. subsp. inflexus

Juncus jacquinii L.

Juncus littoralis C.A.Mey.

Juncus maritimus Lam.

Juncus minutulus (Albert \& Jahand.) Prain.

Juncus pygmaeus Rich. ex Thuill.

Juncus ranarius Songeon \& E.P.Perrier

Juncus rigidus Desf.

Juncus sorrentinii Parl.

Juncus sphaerocarpus Nees

Juncus squarrosus $\mathrm{L}$.

Juncus striatus Schousb. ex E.Mey.

Juncus subnodulosus Schrank

Juncus subulatus Forssk.

Juncus tenageia L.f. subsp. tenageia

Juncus thomasii Ten.

Juncus tingitanus Maire \& Weiller

Juncus triglumis L. subsp. triglumis

Juncus valvatus Link

Luzula alpina Hoppe

Luzula alpinopilosa (Chaix) Breistr. subsp. alpinopilosa

E Luzula calabra Ten.

Luzula campestris (L.) DC. subsp. campestris

Luzula congesta (Thuill.) Lej.

Luzula divulgata Kirschner

Luzula divulgatiformis Bačič \& Jogan

Luzula exspectata Bačič \& Jogan

Luzula forsteri (Sm.) DC.

Luzula glabrata (Hoppe) Desv.

Luzula lutea (All.) DC. subsp. Iutea

Luzula luzulina (Vill.) Racib.

Luzula luzuloides (Lam.) Dandy \& Wilmott subsp. luzuloides

Luzula luzuloides (Lam.) Dandy \& Wilmott subsp. rubella (Hoppe ex Mert. \& W.D.J.Koch) Holub

Luzula multiflora (Ehrh.) Lej. subsp. multiflora

Luzula nivea (Nathh.) DC.

Luzula nutans (Vill.) Duval-Jouve

Luzula pedemontana Boiss. \& Reut.

Luzula pilosa (L.) Willd.

Luzula pindica (Hausskn.) Chrtek \& Křísa

Luzula spicata (L.) DC. subsp. bulgarica (Chrtek \& Křísa) Gamisans

Luzula spicata (L.) DC. subsp. conglomerata (W.D.J.Koch) Murr

Luzula spicata (L.) DC. subsp. italica (Parl.) Arcang.

Luzula spicata (L.) DC. subsp. spicata

Luzula sudetica (Willd.) Schult.

E Luzula sylvatica (Huds.) Gaudin subsp. sicula (Parl.) K.Richt.

Luzula sylvatica (Huds.) Gaudin subsp. sieberi (Tausch) K.Richt.

Luzula sylvatica (Huds.) Gaudin subsp. sylvatica

Luzula taurica (V.I.Krecz.) Novikov

Oreojuncus monanthos (Jacq.) Záv.Drábk. \& Kirschner

Oreojuncus trifidus (Jacq.) Záv.Drábk. \& Kirschner 
Cyperaceae

Taxonomic references: Schoenoplectiella Lye (Lye 2003; Shiels et al. 2014; Glon et al. 2017).

Blysmus compressus (L.) Panz. ex Link

Bolboschoenus glaucus (Lam.) S.G.Sm.

Bolboschoenus laticarpus Marhold, Hroudová, Ducháček \& Zákr.

Bolboschoenus maritimus (L.) Palla

Bolboschoenus planiculmis (F.W.Schmidt) T.V.Egorova

Bulbostylis cioniana (Pi.Savi) Lye - Note: Possibly extinct in Italy (D'Antraccoli et al. 2017).

Carex acuta L.

Carex acutiformis Ehrh.

Carex alba Scop.

Carex appropinquata Schumach.

Carex aterrima Hoppe

Carex atrata L.

Carex atrofusca Schkuhr

Carex austroalpina Bech.

Carex baldensis $\mathrm{L}$.

Carex bicolor All.

Carex bohemica Schreb.

Carex brachystachys Schrank

Carex brizoides $\mathrm{L}$.

Carex brunnescens (Pers.) Poir.

Carex buekii Wimm.

Carex buxbaumii Wahlenb.

Carex canescens $\mathrm{L}$.

Carex capillaris L. subsp. capillaris

Carex capitata $\mathrm{L}$.

Carex caryophyllea Latourr.

Carex chordorrhiza L.f.

Carex colchica J.Gay

Carex curvula All. subsp. curvula

Carex curvula All. subsp. rosae Gilomen

Carex davalliana $\mathrm{Sm}$.

Carex demissa Hornem.

Carex depauperata Curtis ex With.

Carex depressa Link subsp. basilaris (Jord.) Cif. \& Giacom.

Carex diandra Schrank

Carex digitata $\mathrm{L}$.

Carex dioica $\mathrm{L}$.

Carex distachya Desf.

Carex distans $\mathrm{L}$.

Carex disticha Huds.

Carex divisa Huds.

Carex divulsa Stokes

Carex echinata Murray

Carex elata All. subsp. elata

Carex elongata $\mathrm{L}$.

Carex ericetorum Pollich

Carex extensa Gooden.

Carex ferruginea Scop.

Carex fimbriata Schkuhr

Carex firma Host

Carex flacca Schreb. subsp. erythrostachys (Hoppe) Holub

Carex flacca Schreb. subsp. flacca

Carex flava $\mathrm{L}$.

Carex foetida All.

Carex frigida All.

Carex fritschii Waisb.

Carex fuliginosa Schkuhr subsp. fuliginosa

Carex grioletii Roem.

Carex halleriana Asso

Carex hartmanii Cajander

Carex heleonastes L.f.

Carex hirta L.

Carex hispida Willd.

Carex hostiana DC.

Carex humilis Leyss.

Carex illegitima Ces.

Carex kitaibeliana Degen ex Bech.

Carex lachenalii Schkuhr

Carex laevigata Sm.

Carex lasiocarpa Ehrh.

Carex laxula Tin. ex Boott

Carex leersii F.W.Schultz

Carex lepidocarpa Tausch subsp. lepidocarpa

Carex leporina $\mathrm{L}$.

Carex limosa L.

Carex liparocarpos Gaudin subsp. liparocarpos 
Carex macrolepis DC.

Carex macrostachys Bertol.

Carex magellanica Lam. subsp. irrigua (Wahlenb.) Hiitonen

Carex mairei Coss. \& Germ.

Carex maritima Gunnerus

Carex melanostachya Willd.

Carex michelii Host

Carex microcarpa Bertol. ex Moris

Carex microglochin Wahlenb.

Carex montana $\mathrm{L}$.

Carex mucronata All.

Carex muricata $\mathrm{L}$.

Carex myosuroides Vill.

Carex nigra (L.) Reichard subsp. intricata (Tineo) Rivas Mart.

Carex nigra (L.) Reichard subsp. nigra

Carex norvegica Retz.

Carex oedipostyla Duval-Jouve

Carex olbiensis Jord.

Carex ornithopoda Willd.

Carex ornithopodioides Hausm.

Carex otrubae Podp

Carex pairae F.W.Schultz

Carex pallescens $\mathrm{L}$.

Carex panicea $\mathrm{L}$.

Carex paniculata L. subsp. paniculata

Carex panormitana Guss.

Carex parviflora Host

Carex pauciflora Lightf.

Carex pediformis C.A.Mey. subsp. pediformis

Carex pendula Huds.

Carex phyllostachys C.A.Mey.

Carex pilosa Scop.

Carex pilulifera L. subsp. pilulifera

Carex praecox Schreb.

Carex pseudocyperus L.

Carex pulicaris $\mathrm{L}$.

Carex punctata Gaudin

Carex randalpina B.Walln.

Carex remota $\mathrm{L}$.

Carex repens Bellardi

Carex riparia Curtis

Carex rostrata Stokes

Carex rupestris All.

Carex sempervirens Vill. subsp. sempervirens

Carex simpliciuscula Wahlenb.

Carex spicata Huds.

Carex stenophylla Wahlenb. subsp. stenophylla

Carex strigosa Huds.

Carex supina Wahlenb.

Carex sylvatica Huds.

Carex tomentosa $\mathrm{L}$

Carex umbrosa Host subsp. umbrosa

Carex vaginata Tausch

Carex vesicaria $L$.

Carex viridula Michx.

Carex vulpina $\mathrm{L}$.

Cladium mariscus (L.) Poh

Cyperus alopecuroides Rottb.

Cyperus badius Desf.

Cyperus capitatus Vand.

Cyperus distachyos All.

Cyperus flavescens $\mathrm{L}$.

Cyperus flavidus Retz.

Cyperus fuscus $\mathrm{L}$.

Cyperus glaber L.

Cyperus laevigatus L.

Cyperus longus $\mathrm{L}$.

Cyperus michelianus (L.) Delile

Cyperus polystachyos Rottb.

Cyperus rotundus $\mathrm{L}$.

Eleocharis acicularis (L.) Roem. \& Schult.

Eleocharis caduca (Delile) Schult.

Eleocharis carniolica W.D.J.Koch

Eleocharis mamillata (H.Lindb.) H.Lindb. subsp. austriaca (Hayek) Strandh.

Eleocharis mamillata (H.Lindb.) H.Lindb. subsp. mamillata

Eleocharis multicaulis (Sm.) Desv.

Eleocharis ovata (Roth) Roem. \& Schult.

Eleocharis palustris (L.) Roem. \& Schult. subsp. palustris 
Eleocharis parvula (Roem. \& Schult.) Link ex Bluff, Nees \& Schauer

Eleocharis quinqueflora (Hartmann) O.Schwarz

Eleocharis uniglumis (Link) Schult.

Eriophorum angustifolium Honck. subsp. angustifolium

Eriophorum gracile W.D.J.Koch ex Roth

Eriophorum Iatifolium Hoppe

Eriophorum scheuchzeri Hoppe

Eriophorum vaginatum $\mathrm{L}$.

Fimbristylis bisumbellata (Forssk.) Bubani

Fimbristylis dichotoma (L.) Vahl

Fimbristylis squarrosa Vahl

Fuirena pubescens (Poir.) Kunth

Isolepis cernua (Vahl) Roem. \& Schult.

Isolepis fluitans (L.) R.Br.

Isolepis pseudosetacea (Daveau) Gand.

Isolepis setacea (L.) R.Br.

Rhynchospora alba (L.) Vahl

Rhynchospora fusca (L.) W.T.Aiton

Schoenoplectiella mucronata (L.) J.Jung \& H.K.Choi

Schoenoplectiella supina (L.) Lye

Schoenoplectus lacustris (L.) Palla - Note: Schoenoplectus xcarinatus (Sm.) Palla (S. lacustris (L.) Palla $\times$ S. triqueter (L.) Palla) is also recorded for Italy.

Schoenoplectus litoralis (Schrad.) Palla

Schoenoplectus pungens (Vahl) Palla

Schoenoplectus tabernaemontani (C.C.Gmel.) Palla

Schoenoplectus triqueter (L.) Palla

Schoenus ferrugineus $\mathrm{L}$.

Schoenus nigricans $\mathrm{L}$.

Scirpoides holoschoenus (L.) Soják

Scirpus radicans Schkuhr

Scirpus sylvaticus $\mathrm{L}$.

Trichophorum alpinum (L.) Pers.

Trichophorum cespitosum (L.) Hartm. subsp. cespitosum

Trichophorum pumilum (Vahl) Schinz \& Thell.

Poaceae

Taxonomic references: Festuca L. (incl. Ctenopsis De Not., Micropyrum (Gaudin) Link, Psilurus Trin., Vulpia C.C.Gmel.) (Soreng et al. 2017); Lolium L. (Ardenghi and Foggi 2015; Banfi et al. 2018); Triticum L. (Perrino et al. 2014); according to Kellogg (2015) and Saarela et al. (2017), Avellinia Parl., Gaudinia P.Beauv., Koeleria Pers. and Rostraria Trin. should be included in Trisetaria Forssk. as well as Trisetum Pers. We currently have cosidered only Trisetum within Trisetaria according to Soldano (1993), Banfi and Soldano (1996) and Banfi (2000).

Achnatherum bromoides (L.) P.Beauv. - Note: The nomenclature follows the phylogenetic classification proposed by Soreng et al. (2017).

Achnatherum calamagrostis (L.) P.Beauv.

Achnatherum virescens (Trin.) Banfi, Galasso \& Bartolucci - Note: For the nomenclature of this species, see Banfi et al. (2018).

Aeluropus lagopoides (L.) Trin. ex Thwaites

Aeluropus littoralis (Gouan) Parl. subsp. littoralis

Agropyron pectiniforme Roem. \& Schult.

Agrostis alpina Scop.

ET Agrostis canina L. subsp. aspromontana Brullo, Scelsi \& Spamp.

Agrostis canina L. subsp. canina

ET Agrostis canina L. subsp. monteluccii Selvi

Agrostis capillaris L. subsp. capillaris

Agrostis castellana Boiss. \& Reut.

Agrostis curtisii Kerguélen

Agrostis gigantea Roth subsp. gigantea

Agrostis linkii Banfi, Galasso \& Bartolucci subsp. linkii - Note: For the nomenclature of this species, see Banfi et al. (2018).

Agrostis pourretii Willd.

Agrostis rupestris All. subsp. rupestris

Agrostis schleicheri Jord. \& Verl.

Agrostis schraderiana Bech.

Agrostis stolonifera L. subsp. maritima (Lam.) Vasc.

Agrostis stolonifera L. subsp. scabriglumis (Boiss. \& Reut.) Maire

Agrostis stolonifera L. subsp. stolonifera

Agrostis vinealis Schreb.

Aira caryophyllea $\mathrm{L}$.

Aira cupaniana Guss.

Aira elegantissima Schur subsp. elegantissima

Aira intermedia Guss.

Aira multiculmis Dumort.

Aira praecox L.

Aira provincialis Jord.

Aira tenorei Guss.

Airopsis tenella (Cav.) Coss. \& Durieu

Alopecurus aequalis Sobol.

Alopecurus arundinaceus Poir. subsp. arundinaceus

Alopecurus bulbosus Gouan subsp. bulbosus

Alopecurus geniculatus $\mathrm{L}$.

Alopecurus gerardi Vill.

Alopecurus myosuroides Huds. subsp. myosuroides 
Alopecurus pratensis L. subsp. pratensis

Alopecurus rendlei Eig

Ampelodesmos mauritanicus (Poir.) T.Durand \& Schinz

Andropogon distachyos $\mathrm{L}$.

Anisantha diandra (Roth) Tutin ex Tzvelev

Anisantha fasciculata (C.Presl) Nevski subsp. fasciculata

Anisantha macranthera (Hack. ex Trab.) P.Silva

Anisantha madritensis (L.) Nevski subsp. madritensis

Anisantha rigida (Roth) Hyl.

Anisantha rubens (L.) Nevski

Anisantha sterilis (L.) Nevski

Anisantha tectorum (L.) Nevski

Anthoxanthum aristatum Boiss.

Anthoxanthum australe (Schrad.) Veldkamp - Note: For the nomenclature of this species, see Soreng et al. (2017).

Anthoxanthum gracile Biv.

Anthoxanthum nipponicum Honda

Anthoxanthum nitens (Weber) Y.Schouten \& Veldkamp - Note: For the nomenclature of this species, see Soreng et al. (2017).

Anthoxanthum odoratum $\mathrm{L}$.

Anthoxanthum ovatum Lag.

Antinoria insularis Parl.

Apera interrupta (L.) P.Beauv.

Apera spica-venti (L.) P.Beauv. subsp. spica-venti

Aristida adscensionis L. subsp. coerulescens (Desf.) Auquier \& J.Duvign.

Arrhenatherum album (Vahl) Clayton

Arrhenatherum elatius (L.) P.Beauv. ex J.Presl \& C.Presl subsp. bulbosum (Willd.) Schübl. \& G.Martens

Arrhenatherum elatius (L.) P.Beauv. ex J.Presl \& C.Presl subsp. elatius

E Arrhenatherum elatius (L.) P.Beauv. ex J.Presl \& C.Presl subsp. nebrodense (Brullo, Miniss. \& Spamp.) Giardina \& Raimondo

Arrhenatherum elatius (L.) P.Beauv. ex J.Presl \& C.Presl subsp. sardoum (Em.Schmid) Gamisans

Arundo donaciformis (Loisel.) Hardion, Verlaque \& B.Vila

Arundo micrantha Lam.

Arundo plinii Turra

Avellinia festucoides (Link) Valdés \& H.Scholz

Avena barbata Pott ex Link

Avena clauda Durieu

Avena insularis Ladiz.

Avena longiglumis Durieu

Avena lusitanica (Tab.Morais) B.R.Baum

Avena saxatilis (Lojac.) Rocha Afonso

Avena wiestii Steud.

Avenella flexuosa (L.) Drejer subsp. flexuosa

Avenula pubescens (Huds.) Dumort. subsp. Iaevigata (Schur) Holub

Avenula pubescens (Huds.) Dumort. subsp. pubescens

Beckmannia eruciformis (L.) Host subsp. eruciformis

E Bellardiochloa variegata (Lam.) Kerguélen subsp. aetnensis (C.Presl) Giardina \& Raimondo

E Bellardiochloa variegata (Lam.) Kerguélen subsp. nebrodensis (Asch. \& Graebn.) C.Brullo, Brullo \& Giusso

Bellardiochloa variegata (Lam.) Kerguélen subsp. variegata

Bothriochloa insculpta (A.Rich.) A.Camus subsp. panormitana (Parl.) Giardina \& Raimondo

Bothriochloa ischaemum (L.) Keng

Brachypodium distachyon (L.) P.Beauv.

E Brachypodium genuense (DC.) Roem. \& Schult.

Brachypodium glaucovirens (Murb.) T.Durand \& B.D.Jacks.

Brachypodium hybridum Catalán, Joch.Müll., Hasterok \& Jenkins - Note: For the nomenclature and distribution of this species, see Catalán et al. (2012, 2016).

Brachypodium phoenicoides (L.) Roem. \& Schult.

Brachypodium pinnatum (L.) P.Beauv.

Brachypodium retusum (Pers.) P.Beauv.

Brachypodium rupestre (Host) Roem. \& Schult.

Brachypodium stacei Catalán, Joch.Müll., Mur \& Langdon - Note: For the nomenclature and distribution of this species, see Catalán et al. (2012, 2016).

Brachypodium sylvaticum (Huds.) P.Beauv.

Briza maxima L.

Briza media L.

Briza minor L.

Bromopsis benekenii (Lange) Holub

E Bromopsis caprina (A.Kern. ex Hack.) Banfi \& N.G.Passal.

Bromopsis condensata (Hack.) Holub subsp. condensata

Bromopsis condensata (Hack.) Holub subsp. microtricha (Borbás) Jogan \& Bačič

Bromopsis erecta (Huds.) Fourr. subsp. erecta

Bromopsis erecta (Huds.) Fourr. subsp. stenophylla (Link) H.Scholz \& Valdés

Bromopsis erecta (Huds.) Fourr. subsp. transsilvanica (Steud.) H.Scholz \& Valdés

Bromopsis pannonica (Kumm. \& Sendtn.) Holub subsp. pannonica

DD Bromopsis pumpelliana (Scribn.) Holub

Bromopsis ramosa (Huds.) Holub subsp. ramosa

Bromus alopecuros Poir. subsp. alopecuros

Bromus arvensis L. subsp. arvensis

Bromus arvensis L. subsp. segetalis H.Scholz

Bromus commutatus Schrad. subsp. commutatus

Bromus commutatus Schrad. subsp. decipiens (Bomble \& H.Scholz) H.Scholz

Bromus commutatus Schrad. subsp. neglectus (Parl.) P.M.Sm. 
Bromus grossus Desf. ex DC. - Note: Possibly extinct in Italy (Rossi et al. 2013).

Bromus hordeaceus L. subsp. hordeaceus

Bromus hordeaceus L. subsp. Iongipedicellatus Spalton

Bromus hordeaceus L. subsp. molliformis (J.Lloyd ex Billot) Maire \& Weiller

Bromus hordeaceus L. subsp. pseudothominei (P.M.Sm.) H.Scholz

Bromus hordeaceus L. subsp. thominei (Hardouin) Braun-Blanq.

Bromus intermedius Guss.

Bromus japonicus Thunb. subsp. anatolicus (Boiss. \& Heldr.) Pénzes

Bromus japonicus Thunb. subsp. japonicus

Bromus japonicus Thunb. subsp. subsquarrosus (Borbás) Pénzes

Bromus lanceolatus Roth

Bromus parvispiculatus $\mathrm{H} . \mathrm{Scholz}$

Bromus racemosus $L$. subsp. racemosus

Bromus scoparius L.

Bromus secalinus $L$.

Bromus squarrosus $L$. subsp. squarrosus

Calamagrostis arenaria (L.) Roth subsp. arundinacea (Husn.) Banfi, Galasso \& Bartolucci - Note: For the nomenclature of this taxon, see Banfi et al. (2018).

Calamagrostis arundinacea (L.) Roth

Calamagrostis canescens (Weber) Roth subsp. canescens

Calamagrostis corsica (Hack. ex Briq.) Prain

Calamagrostis epigejos (L.) Roth subsp. epigejos

Calamagrostis pseudophragmites (Haller f.) Koeler subsp. pseudophragmites

Calamagrostis varia (Schrad.) Host

Calamagrostis villosa (Chaix) J.F.Gmel.

Castellia tuberculosa (Moris) Bor

Catabrosa aquatica (L.) P.Beauv.

Catapodium balearicum (Willk.) H.Scholz

Catapodium hemipoa (Delile ex Spreng.) M.Laínz

Catapodium pauciflorum (Merino) Brullo, Giusso, Miniss. \& Spamp.

Catapodium rigidum (L.) C.E.Hubb. subsp. majus (C.Presl) F.H.Perring \& P.D.Sell

Catapodium rigidum (L.) C.E.Hubb. subsp. rigidum

Catapodium zwierleinii (Lojac.) Brullo

Cenchrus ciliaris L.

Chrysopogon gryllus (L.) Trin.

Cleistogenes serotina (L.) Keng subsp. serotina

Coleanthus subtilis (Tratt.) Seidl - Note: This species is recorded only for TAA, but it lacks recent confirmations. According to Rossi et al. (2013), C. subtilis is possibly extinct in Italy.

Cornucopiae cucullatum $\mathrm{L}$.

Corynephorus articulatus (Desf.) P.Beauv.

Corynephorus canescens (L.) P.Beauv.

Corynephorus divaricatus (Pourr.) Breistr.

Cynodon dactylon (L.) Pers.

Cynosurus cristatus $\mathrm{L}$.

Cynosurus echinatus $L$.

Cynosurus effusus Link

Cynosurus polybracteatus Poir.

Dactylis glomerata L. subsp. glomerata

Dactylis glomerata L. subsp. hackelii (Asch. \& Graebn.) Cif. \& Giacom.

Dactylis glomerata L. subsp. hispanica (Roth) Nyman

Dactylis glomerata L. subsp. Iobata (Drejer) H.Lindb.

Dactylis glomerata L. subsp. reichenbachii (Dalla Torre \& Sarnth.) Stebbins \& D.Zohary

Dactylis glomerata L. subsp. slovenica (Domin) Domin

Danthonia alpina Vest

Danthonia decumbens (L.) DC. subsp. decumbens

Dasypyrum villosum (L.) P.Candargy

Deschampsia cespitosa (L.) P.Beauv. subsp. cespitosa

DD Deschampsia cespitosa (L.) P.Beauv. subsp. gaudinii K.Richt.

Deschampsia cespitosa (L.) P.Beauv. subsp. parviflora (Thuill.) Dumort.

Deschampsia cespitosa (L.) P.Beauv. subsp. rhenana (Gremli) Kerguélen

Deschampsia media (Gouan) Roem. \& Schult. subsp. media

Desmazeria pignattii Brullo \& Pavone

Desmazeria sicula (Jacq.) Dumort.

Digitaria debilis (Desf.) Willd.

Digitaria ischaemum (Schreb. ex Schweigg.) Schreb. ex Muhl. subsp. ischaemum

Digitaria sanguinalis (L.) Scop.

Drymochloa drymeja (Mert. \& W.D.J.Koch) Holub subsp. exaltata (C.Presl) Foggi \& Signorini

Drymochloa sylvatica (Pollich) Holub

Echinaria capitata (L.) Desf.

Echinochloa crus-galli (L.) P.Beauv. subsp. crus-galli

Elymus acutus (DC.) M.A.Thiébaud

Elymus caninus (L.) L.

Elymus elongatus (Host) Runemark subsp. elongatus

Elymus farctus (Viv.) Runemark ex Melderis

Elymus flaccidifolius (Boiss. \& Heldr.) Melderis

Elymus hispidus (Opiz) Melderis subsp. barbulatus (Schur) Melderis

Elymus hispidus (Opiz) Melderis subsp. hispidus

Elymus hispidus (Opiz) Melderis subsp. pouzolzii (Godr.) Melderis 
Elymus panormitanus (Parl.) Tzvelev

Elymus repens (L.) Gould subsp. repens

Eragrostis barrelieri Daveau subsp. barrelieri

Eragrostis cilianensis (All.) Vignolo ex Janch. subsp. cilianensis

Eragrostis minor Host subsp. minor

Eragrostis pilosa (L.) P.Beauv. subsp. pilosa

Festuca acuminata Gaudin

E Festuca alfrediana Foggi \& Signorini subsp. alfrediana

E Festuca alfrediana Foggi \& Signorini subsp. ferrariniana Foggi, Parolo \& Gr.Rossi

Festuca alopecuros Schousb.

Festuca alpestris Roem. \& Schult.

Festuca alpina Suter

E Festuca apuanica Markgr.-Dann.

Festuca arvernensis Auquier, Kerguélen \& Markgr.-Dann. subsp. costei (St.-Yves) Auquier \& Kerguélen

Festuca austrodolomitica Pils \& Prosser

Festuca bauzanina (Pils) S.Arndt subsp. bauzanina

Festuca bauzanina (Pils) S.Arndt subsp. rhaetica S.Arndt

Festuca billyi Kerguélen \& Plonka

Festuca bosniaca Kumm. \& Sendtn. subsp. bosniaca

Festuca bromoides $L$.

Festuca calva (Hack.) K.Richt.

Festuca centroapenninica (Markgr.-Dann.) Foggi, F.Conti \& Pignatti

Festuca cinerea Vill.

Festuca circummediterranea Patzke

Festuca cyrnea (Litard. \& St.-Yves) Signorini, Foggi \& Nardi

Festuca danthonii Asch. \& Graebn. Subsp. danthonii

Festuca divaricata Desf.

Festuca fasciculata Forssk.

Festuca filiformis Pourr.

Festuca flavescens Bellardi

Festuca gamisansii Kerguélen subsp. aethaliae Signorini \& Foggi

Festuca geniculata (L.) Lag. \& Rodr. subsp. geniculata

Festuca guinochetii (Bidault) S.Arndt

Festuca gypsophila Hack.

Festuca halleri All. subsp. halleri

Festuca heteromalla Pourr.

Festuca heterophylla Lam.

E Festuca humifusa Brullo \& Guarino

ET Festuca imperatrix Catonica

Festuca incurva (Gouan) Gutermann

Festuca inops De Not.

Festuca intercedens (Hack.) Lüdi ex Bech.

Festuca jeanpertii (St.-Yves) Markgr.-Dann. subsp. campana (N.Terracc.) Markgr.-Dann.

Festuca lachenalii (C.C.Gmel.) Spenn.

Festuca laevigata Gaudin

Festuca lanceolata Forssk.

Festuca ligustica (All.) Bertol.

Festuca luedii (Markgr.-Dann.) Foggi, Gr.Rossi, Parolo \& Wallossek

Festuca marginata (Hack.) K.Richt. subsp. gallica (Hack.) Breistr. ex Ardenghi \& Foggi

Festuca maritima $\mathrm{L}$.

E Festuca morisiana Parl. subsp. morisiana

E Festuca morisiana Parl. subsp. sicula Cristaudo, Foggi, Galesi \& Maugeri

Festuca muralis Kunth

Festuca myuros L. subsp. myuros

Festuca nigricans (Hack.) K.Richt.

Festuca nitida Kit. ex Schult. subsp. nitida

Festuca norica (Hack.) K.Richt.

Festuca ovina L. subsp. guestfalica (Boenn. ex Rchb.) K.Richt.

Festuca ovina L. subsp. molinieri (Litard.) O.Bolòs \& Vigo

Festuca picturata Pils

E Festuca pignattiorum Markgr.-Dann

Festuca plonkae Foggi \& Signorini

Festuca pseudodura Steud.

Festuca pseudovaria J.Vetter

Festuca pumila Chaix

Festuca pyramidata Link

E $\quad$ Festuca riccerii Foggi \& Gr.Rossi

Festuca rivularis Boiss. subsp. rivularis

E Festuca robustifolia Markgr.-Dann.

Festuca rubra L. subsp. commutata (Gaudin) Markgr.-Dann.

Festuca rubra L. subsp. juncea (Hack.) K.Richt.

Festuca rubra L. subsp. microphylla St.-Yves

Festuca rubra L. subsp. rubra - Note: This is a doubtfully native taxon, used as a turf grass. Most literature records from natural environments may refer to other subspecies of F. rubra or to F. trichophylla (Ducros ex Gaudin) K.Richt.

Festuca rupicaprina (Hack.) A.Kern.

E Festuca sardoa (Hack.) K.Richt.

Festuca scabriculmis (Hack.) K.Richt.

Festuca sicula C.Presl 
Festuca stenantha (Hack.) K.Richt.

Festuca stricta Host subsp. sulcata (Hack.) Patzke ex Pils

Festuca stricta Host subsp. trachyphylla (Hack.) Patzke ex Pils

Festuca ticinensis (Markgr.-Dann.) Markgr.-Dann.

Festuca trichophylla (Ducros ex Gaudin) K.Richt. subsp. asperifolia (St.-Yves) Al-Bermani

Festuca trichophylla (Ducros ex Gaudin) K.Richt. subsp. trichophylla

Festuca valesiaca Schleich. ex Gaudin subsp. valesiaca

E Festuca veneris Gr.Rossi, Foggi \& Signorini

E Festuca violacea Ser. ex Gaudin subsp. italica Foggi, Gr.Rossi \& Signorini

E Festuca violacea Ser. ex Gaudin subsp. puccinellii (Parl.) Foggi, Gr.Rossi \& Signorini

Festuca violacea Ser. ex Gaudin subsp. violacea

Festuca winnebachensis (Wallossek \& Markgr.-Dann.) Foggi, Gr.Rossi, Parolo \& Wallossek

Gastridium phleoides (Nees \& Meyen) C.E.Hubb. subsp. phleoides - Note: It is often confused with G. ventricosum (Gouan) Schinz \& Thell.

Gastridium scabrum C.Presl

Gastridium ventricosum (Gouan) Schinz \& Thell.

Gaudinia fragilis (L.) P.Beauv.

Glyceria declinata Bréb.

Glyceria fluitans (L.) R.Br.

Glyceria maxima (Hartm.) Holmb. subsp. maxima

Glyceria notata Chevall.

Glyceria pedicellata F.Towns.

Glyceria spicata Guss.

Hainardia cylindrica (Willd.) Greuter

Helictochloa adsurgens (Simonk.) Romero Zarco subsp. ausserdorferi (Asch. \& Graebn.) Romero Zarco

Helictochloa bromoides (Gouan) Romero Zarco subsp. bromoides

Helictochloa cincinnata (Ten.) Romero Zarco

E Helictochloa praetutiana (Parl. ex Arcang.) Bartolucci, F.Conti, Peruzzi \& Banfi subsp. praetutiana

E Helictochloa praetutiana (Parl. ex Arcang.) Bartolucci, F.Conti, Peruzzi \& Banfi subsp. rigida (Sarfatti) Bartolucci, F.Conti, Peruzzi \& Banfi

Helictochloa praeusta (Rchb.) Romero Zarco subsp. pseudoviolacea (Dalla Torre) H.Scholz

Helictochloa pratensis (L.) Romero Zarco subsp. pratensis - Note: This taxon has been detected once by A. Saint-Yves near Fenestrelle (Torino, Piemonte), while all other records for the Italian Alps are unreliable (Banfi 2017).

Helictochloa versicolor (Vill.) Romero Zarco subsp. versicolor

Helictotrichon convolutum (C.Presl) Henrard

Helictotrichon parlatorei (Woods) Pilg.

Helictotrichon petzense Melzer

Helictotrichon sedenense (Clarion ex DC.) Holub subsp. sedenense

Helictotrichon sempervirens (Vill.) Pilg.

Helictotrichon setaceum (Vill.) Henrard

Hemarthria altissima (Poir.) Stapf \& C.E.Hubb.

Heteropogon contortus (L.) P.Beauv. ex Roem. \& Schult.

Holcus annuus C.A.Mey. subsp. setiglumis (Boiss. \& Reut) M.Seq. \& Castrov.

Holcus lanatus L. subsp. Ianatus

Holcus mollis L. subsp. mollis

ET Holcus notarisii Nyman - Note: Since this species was only recorded for its locus classicus, where it has never been found again, we can suspect that it just represents a teratological expression of H. mollis (widespread in the same habitat), rather than a truly distinct species.

Hordelymus europaeus (L.) Harz

Hordeum bulbosum $\mathrm{L}$.

Hordeum geniculatum All.

Hordeum marinum Huds.

Hordeum murinum L. subsp. glaucum (Steud.) Tzvelev

Hordeum murinum L. subsp. leporinum (Link) Arcang.

Hordeum murinum L. subsp. murinum

Hordeum secalinum Schreb.

Hyparrhenia hirta (L.) Stapf subsp. hirta

Hyparrhenia sinaica (Delile) Llauradó ex G.López

Imperata cylindrica (L.) Raeusch.

Koeleria australis A.Kern.

Koeleria callieri (Domin) Ujhelyi

Koeleria cenisia Reut. ex E.Rev.

Koeleria eriostachya Pančić

Koeleria hirsuta Gaudin

T Koeleria insubrica Brullo, Giusso \& Miniss.

ET Koeleria lucana Brullo, Giusso \& Miniss.

Koeleria macrantha (Ledeb.) Schult. subsp. macrantha

Koeleria pyramidata (Lam.) P.Beauv.

E Koeleria splendens C.Presl

Koeleria subcaudata (Asch. \& Graebn.) Ujhelyi

Koeleria vallesiana (Honck.) Gaudin subsp. alpicola (Gren. \& Godr.) Asch. \& Graebn.

Koeleria vallesiana (Honck.) Gaudin subsp. vallesiana

Lagurus ovatus L. subsp. nanus (Guss.) Messeri

Lagurus ovatus $L$. subsp. ovatus

Lagurus ovatus L. subsp. vestitus (Messeri) Brullo

Lamarckia aurea (L.) Moench

Leersia oryzoides (L.) Sw.

E Leucopoa calabrica (Huter, Porta \& Rigo) H.Scholz \& Foggi

Leucopoa dimorpha (Guss.) H.Scholz \& Foggi

Leucopoa laxa (Host) H.Scholz \& Foggi

Leucopoa pulchella (Schrad.) H.Scholz \& Foggi subsp. jurana (Gren.) H.Scholz \& Foggi 
Leucopoa pulchella (Schrad.) H.Scholz \& Foggi subsp. pulchella

Leucopoa spectabilis (Jan ex Bertol.) H.Scholz \& Foggi subsp. carniolica (Hack.) H.Scholz \& Foggi

Leucopoa spectabilis (Jan ex Bertol.) H.Scholz \& Foggi subsp. croatica (A.Kern.) Foggi, Parolo, Gr.Rossi, Ardenghi \& Quercioli

Leucopoa spectabilis (Jan ex Bertol.) H.Scholz \& Foggi subsp. spectabilis

Lolium apenninum (De Not.) Ardenghi \& Foggi

Lolium arundinaceum (Schreb.) Darbysh. subsp. arundinaceum

Lolium giganteum (L.) Darbysh.

Lolium interruptum (Desf.) Banfi, Galasso, Foggi, Kopecký \& Ardenghi subsp. corsicum (Hack.) Banfi, Galasso, Kopecký \& Ardenghi

Lolium interruptum (Desf.) Banfi, Galasso, Foggi, Kopecký \& Ardenghi subsp. interruptum

Lolium multiflorum Lam.

Lolium perenne $\mathrm{L}$.

Lolium pluriflorum (Schult.) Banfi, Galasso, Foggi, Kopecký \& Ardenghi

Lolium pratense (Huds.) Darbysh.

Lolium rigidum Gaudin subsp. lepturoides (Boiss.) Sennen \& Mauricio

Lolium rigidum Gaudin subsp. rigidum

Lolium siculum Parl.

Lygeum spartum L.

E Megathyrsus bivonanus (Brullo, Miniss., Scelsi \& Spamp.) Verloove

Melica amethystina Pourr.

Melica ciliata L. subsp. ciliata

Melica ciliata L. subsp. magnolii (Godr. \& Gren.) K.Richt.

Melica cupanii Guss. subsp. cupanii

Melica minuta L. subsp. Iatifolia (Coss.) W. Hempel

Melica minuta L. subsp. minuta

Melica nutans $\mathrm{L}$.

Melica picta K.Koch

Melica transsilvanica Schur subsp. klokovii Tzvelev

Melica transsilvanica Schur subsp. transsilvanica - Note: According to Hempel (2011), both M. transsilvanica subsp. klokovii and M. transsilvanica s.str. occur in Italy. The northern populations constantly show character states typical of $M$. transsilvanica subsp. klokovii, albeit in this area only the autonymic subspecies should occur.

Melica uniflora Retz.

Mibora minima (L.) Desv.

Milium effusum L. subsp. alpicola Chrtek

Milium effusum L. subsp. effusum

Milium montianum (Parl.) K.Richt.

Milium vernale M.Bieb. subsp. scabrum (Rich.) K.Richt.

Milium vernale $M . B i e b$. subsp. vernale

Molineriella minuta (L.) Rouy

Molinia arundinacea Schrank

Molinia caerulea (L.) Moench

Moorochloa eruciformis (Sm.) Veldkamp

Nardus stricta L.

Oloptum miliaceum (L.) Röser \& H.R.Hamasha

Oloptum thomasii (Duby) Banfi \& Galasso

Oplismenus undulatifolius (Ard.) P.Beauv.

Oreochloa disticha (Wulfen) Link subsp. disticha

Oreochloa ses/erioides (All.) K.Richt.

Panicum repens $\mathrm{L}$.

Parapholis filiformis (Roth) C.E.Hubb.

Parapholis incurva (L.) C.E.Hubb. subsp. incurva

Parapholis marginata Runemark

Parapholis pycnantha (Hack.) C.E.Hubb.

Parapholis strigosa (Dumort.) C.E.Hubb.

Parvotrisetum myrianthum (Bertol.) Chrtek

Patzkea coerulescens (Desf.) H.Scholz

Patzkea paniculata (L.) G.H.Loos subsp. paniculata

Phalaris aquatica $\mathrm{L}$.

Phalaris arundinacea $L$. subsp. arundinacea

E Phalaris arundinacea L. subsp. rotgesii (Husn.) Kerguélen

Phalaris brachystachys Link

Phalaris coerulescens Desf.

Phalaris elongata Braun-Blanq.

Phalaris minor Retz.

Phalaris paradoxa $\mathrm{L}$.

Phalaris truncata Guss. ex Bertol.

Phleum alpinum $\mathrm{L}$.

Phleum arenarium L. subsp. caesium $\mathrm{H}$.Scholz

Phleum echinatum Host

Phleum exaratum Hochst. ex Griseb. subsp. exaratum

Phleum hirsutum Honck. subsp. ambiguum (Ten.) Cif. \& Giacom.

Phleum hirsutum Honck. subsp. hirsutum

Phleum nodosum $\mathrm{L}$.

Phleum paniculatum Huds. subsp. paniculatum

Phleum phleoides (L.) H.Karst.

Phleum pratense $\mathrm{L}$. subsp. pratense

Phleum rhaeticum (Humphries) Rauschert

Phleum sardoum (Hack.) Hack.

Phleum subulatum (Savi) Asch. \& Graebn. subsp. subulatum 
Pholiurus pannonicus (Host) Trin.

Phragmites australis (Cav.) Trin. ex Steud. subsp. altissimus (Benth.) Clayton

Phragmites australis (Cav.) Trin. ex Steud. subsp. australis

Piptatherum coerulescens (Desf.) P.Beauv.

Poa alpina L. subsp. alpina

Poa angustifolia L.

Poa annua $\mathrm{L}$.

Poa badensis Haenke ex Willd.

Poa balbisii Parl.

Poa bivonae Parl. ex Guss.

Poa bulbosa L. subsp. bulbosa

Poa bulbosa L. subsp. pseudoconcinna (Schur) Asch. \& Graebn.

Poa cenisia All.

Poa chaixii Vill.

Poa compressa $\mathrm{L}$.

Poa glauca Vahl subsp. glauca

Poa hybrida Gaudin

Poa infirma Kunth

Poa laxa Haenke subsp. Iaxa

Poa minor Gaudin

Poa molinerii Balb.

Poa nemoralis L. subsp. glaucantha (Gaudin) Banfi

Poa nemoralis $L$. subsp. nemoralis

Poa palustris $L$. subsp. palustris

Poa perconcinna J.R.Edm.

Poa perligularis $\mathrm{H} . \mathrm{Scholz}$

Poa pratensis L. subsp. pratensis

Poa pumila Host

Poa remota Forselles

Poa supina Schrad.

Poa sylvicola Guss.

Poa timoleontis Heldr. ex Boiss.

Poa trivialis $\mathrm{L}$.

Polypogon maritimus Willd. subsp. maritimus

Polypogon monspeliensis (L.) Desf.

Polypogon subspathaceus Req.

Polypogon viridis (Gouan) Breistr.

Psilathera ovata (Hoppe) Deyl

Puccinellia distans (Jacq.) Parl. subsp. distans

Puccinellia fasciculata (Torr.) E.P.Bicknell subsp. fasciculata

Puccinellia festuciformis (Host) Parl. subsp. festuciformis

Puccinellia festuciformis (Host) Parl. subsp. lagascana M.A.Juliá \& J.M.Monts.

ET Puccinellia gussonei Parl. - Note: Possibly extinct (Rossi et al. 2013).

Rostraria cristata (L.) Tzvelev

Rostraria hispida (Savi) Doğan - Note: Most of the records from Italy need confirmation because of confusion with R. cristata.

Rostraria pubescens (Lam.) Trin.

Sclerochloa dura (L.) P.Beauv.

Secale strictum (C.Presl) C.Presl subsp. strictum

E Sesleria apennina Ujhelyi

$\mathrm{T} \quad$ Sesleria argentea (Savi) Savi

Sesleria autumnalis (Scop.) F.W.Schultz

Sesleria caerulea (L.) Ard. subsp. angustifolia (Hack. \& Beck) Jogan

Sesleria caerulea (L.) Ard. subsp. caerulea

E Sesleria calabrica (Deyl) Di Pietro

ET Sesleria insularis Sommier subsp. barbaricina Arrigoni

Sesleria insularis Sommier subsp. insularis

ET Sesleria insularis Sommier subsp. morisiana Arrigoni

E Sesleria italica (Pamp.) Ujhelyi - Note: According to Ubaldi (2016), S. italica subsp. mariculensis Ubaldi would differ from the typical Sesleria italica for a number of quantitative character states, mainly concerning panicle length and leaf width. Considering the high morphological variability in the whole genus Sesleria, the continuous variation and the absence of any ecological or geographical discontinuity between the two putative subspecies, it is very difficult to justify their recognition. The study of the type specimens designated by Ujhelyi (1959), kept in BP and Fl, and of living and dried specimens collected in Galeata (locus classicus of $S$. italica) confirmed that the features putatively diagnostic of $S$. italica subsp. mariculensis are also shown by $S$. italica s.str.

Sesleria juncifolia Wulfen ex Suffren subsp. juncifolia

Sesleria kalnikensis Jáv.

E Sesleria nitida Ten. subsp. nitida

ET Sesleria nitida Ten. subsp. sicula Brullo \& Giusso

E Sesleria pichiana Foggi, Gr.Rossi \& Pignotti

ET Sesleria pulchella (Chiosi) Ubaldi - Note: The taxonomic value of this taxon is doubtful. Apparently, S. pulchella is mainly related to S. italica (Pamp.) Ujhelyi, and, at a first glance, it seems simply marked by extremely reduced dimensions. Waiting for further karyological, molecular and biometric studies, we provisionally accept $S$. pulchella as a good species.

ET Sesleria tuzsonii Ujhelyi

Sesleria uliginosa Opiz

Sesleriella leucocephala (DC.) Deyl

Sesleriella sphaerocephala (Ard.) Deyl

C Setaria italica (L.) P.Beauv. subsp. viridis (L.) Thell.

Setaria pumila (Poir.) Roem. \& Schult.

Setaria verticillata (L.) P.Beauv.

Sphenopus divaricatus (Gouan) Rchb. subsp. divaricatus 
Sporobolus aculeatus (L.) P.M.Peterson

Sporobolus alopecuroides (Piller \& Mitterp.) P.M.Peterson

Sporobolus maritimus (Curtis) P.M.Peterson \& Saarela

Sporobolus schoenoides (L.) P.M.Peterson

Sporobolus virginicus (L.) Kunth

Stipa aquilana Moraldo

Stipa austroitalica Martinovský subsp. appendiculata (Čelak.) Moraldo

Stipa austroitalica Martinovský subsp. austroitalica

Stipa austroitalica Martinovský subsp. frentana Moraldo \& Ricceri

Stipa austroitalica Martinovský subsp. theresiae Martinovský \& Moraldo

Stipa barbata Desf. subsp. barbata

Stipa capillata L.

ET Stipa dasyvaginata Martinovský subsp. apenninicola Martinovský \& Moraldo

Stipa epilosa Martinovský

Stipa eriocaulis Borbás subsp. eriocaulis

ET $\quad$ Stipa etrusca Moraldo

Stipa juncea L.

Stipa letourneuxii Trabut subsp. letourneuxii

ET Stipa oligotricha Moraldo subsp. kiemii (Martinovský) Moraldo

ET Stipa oligotricha Moraldo subsp. oligotricha

Stipa pennata L. subsp. pennata

Stipa pulcherrima K.Koch subsp. crassiculmis (P.A.Smirn.) Tzvelev

Stipa pulcherrima K.Koch subsp. pulcherrima

ET Stipa sicula Moraldo, La Valva, Ricciardi \& Caputo

Stipa tirsa Steven subsp. tirsa

ET Stipa valdemonensis Cataldo, S.A.Giardina, Moraldo \& Raimondo

ET Stipa veneta Moraldo

Stipellula capensis (Thunb.) Röser \& H.R.Hamasha

Taeniatherum asperum (Simonk.) Nevski

Taeniatherum caput-medusae (L.) Nevski

Tragus racemosus (L.) All.

Tricholaena teneriffae (L.f.) Link

Tripidium ravennae (L.) H.Scholz subsp. ravennae

Tripidium strictum (Host) H.Scholz

Triplachne nitens (Guss.) Link

Trisetaria alpestris (Host) Baumg.

Trisetaria argentea (Willd.) Banfi, Galasso \& Soldano

Trisetaria aurea (Ten.) Pignatti

E Trisetaria burnoufii (Req. ex Parl.) Banfi \& Soldano

Trisetaria distichophylla (Vill.) Paunero

Trisetaria flavescens (L.) Baumg. subsp. flavescens

Trisetaria flavescens (L.) Baumg. subsp. purpurascens (DC.) Banfi \& Soldano

Trisetaria flavescens (L.) Baumg. subsp. splendens (C.Presl) Banfi \& Soldano

E Trisetaria gracilis (Moris) Banfi \& Arrigoni

Trisetaria loeflingiana (L.) Paunero subsp. loeflingiana

Trisetaria panicea (Lam.) Paunero

Trisetaria segetum (Savi) Soldano

Trisetaria spicata (L.) Paunero subsp. ovatipaniculata (Hultén ex Jonsell) Banfi \& Soldano

E Trisetaria villosa (Bertol.) Banfi \& Soldano

C Triticum biunciale (Vis.) K.Rich.

Triticum neglectum (Req. ex Bertol.) Greuter

C Triticum triunciale (L.) Raspail subsp. triunciale

C Triticum uniaristatum (Vis.) K.Richt.

Triticum vagans (Jord. \& Fourr.) Greuter

C Triticum ventricosum (Tausch) Ces., Pass. \& Gibelli

Ventenata dubia (Leers) Coss.

Vulpiella tenuis (Tineo) Kerguélen

Ceratophyllaceae

Ceratophyllum demersum $\mathrm{L}$.

Ceratophyllum submersum L. subsp. submersum

Berberidaceae

Taxonomic references: Berberis L. (Harber and Raab-Straube 2015).

$E$

Berberis aetnensis C.Presl

Berberis vulgaris $\mathrm{L}$.

Epimedium alpinum $\mathrm{L}$.

CT Gymnospermium scipetarum Paparisto \& Qosja ex E.Mayer \& Pulević

Ranunculaceae

Taxonomic references: Aquilegia L. (Nardi 2015); Ranunculus sect. Batrachium DC. (Desfayes 2011; Wiegleb et al. 2017); Ranunculus auricomus L. group (Dunkel 2010, 2011; Dunkel and Poldini 2016; Conti and Bartolucci 2017); Thalictrum L. (Hand 2001).

Aconitum angustifolium Rchb.

Aconitum anthora $\mathrm{L}$.

Aconitum burnatii Gáyer subsp. burnatii 
Aconitum degenii Gáyer subsp. paniculatum (Arcang.) Mucher

Aconitum degenii Gáyer subsp. valesiacum (Gáyer) Mucher

Aconitum lycoctonum $\mathrm{L}$. emend. Koelle

Aconitum napellus L. emend. Skalický

Aconitum tauricum Wulfen

Aconitum variegatum $L$. subsp. nasutum (Fisch. ex Rchb.) Götz

Aconitum variegatum $L$. subsp. variegatum

Aconitum vitosanum Gáyer

Actaea spicata $\mathrm{L}$.

Adonis aestivalis $L$. subsp. aestivalis

Adonis aestivalis L. subsp. squarrosa (Steven) Nyman

Adonis annua $\mathrm{L}$.

E Adonis distorta Ten.

Adonis flammea Jacq. subsp. cortiana (Jacq.) C.H.Steinb.

Adonis flammea Jacq. subsp. flammea

Adonis microcarpa DC. subsp. microcarpa

Adonis vernalis $\mathrm{L}$.

Anemonastrum narcissiflorum (L.) Holub subsp. narcissiflorum

Anemone apennina $\mathrm{L}$.

Anemone coronaria $\mathrm{L}$.

Anemone hortensis L. subsp. hortensis

Anemone palmata $\mathrm{L}$.

Anemonoides baldensis (L.) Galasso, Banfi \& Soldano

Anemonoides nemorosa (L.) Holub

Anemonoides ranunculoides (L.) Holub

ET Anemonoides trifolia (L.) Holub subsp. brevidentata (Ubaldi \& Puppi) Galasso, Banfi \& Soldano

Anemonoides trifolia (L.) Holub subsp. trifolia

Aquilegia alpina L.

E Aquilegia apuana (Marchetti) E.Nardi

Aquilegia atrata W.D.J.Koch

E Aquilegia barbaricina Arrigoni \& E.Nardi

E Aquilegia bertolonii Schott

E Aquilegia champagnatii Moraldo, E.Nardi \& la Valva

Aquilegia confusa Rota

ET Aquilegia cremnophila Bacch., Brullo, Congiu, Fenu, J.L.Garrido \& Mattana - Note: Although Nardi (2015) considered this plant as a synonym of Aquilegia nuragica, A. cremnophila is morphologically, ecologically and genetically well differentiated from the other Sardinian congeneric taxa (Bacchetta et al. 2012; Garrido et al. 2012; Mattana et al. 2012).

Aquilegia dumeticola Jord.

Aquilegia einseleana F.W.Schultz

E Aquilegia lucensis E.Nardi

E Aquilegia magellensis F.Conti \& Soldano

Aquilegia marcelliana E.Nardi

Aquilegia nugorensis Arrigoni \& E.Nardi

Aquilegia nuragica Arrigoni \& E.Nardi

Aquilegia ophiolithica Barberis \& E.Nardi

Aquilegia reuteri Boiss.

E Aquilegia sicula (Strobl) E.Nardi

Aquilegia vulgaris $\mathrm{L}$.

Callianthemum coriandrifolium Rchb.

E Callianthemum kernerianum Freyn ex A.Kern.

Caltha palustris $\mathrm{L}$.

Ceratocephala falcata (L.) Cramer

Clematis alpina (L.) Mill.

Clematis cirrhosa $\mathrm{L}$.

Clematis flammula $\mathrm{L}$.

Clematis integrifolia L. - Note: Extinct in Italy (Poldini 2009).

Clematis recta $\mathrm{L}$.

E Clematis rigoi W.T.Wang

Clematis vitalba $\mathrm{L}$.

Clematis viticella $\mathrm{L}$.

Delphinium ajacis $\mathrm{L}$.

Delphinium consolida L. subsp. consolida

Delphinium consolida L. subsp. paniculatum (Host) N.Busch

Delphinium dubium (Rouy \& Foucaud) Pawl.

Delphinium elatum $L$. subsp. helveticum Pawl.

Delphinium emarginatum C.Presl subsp. emarginatum

Delphinium fissum Waldst. \& Kit. subsp. fissum

C Delphinium gracile DC.

Delphinium halteratum Sm. subsp. halteratum

E Delphinium longipes Moris

Delphinium peregrinum $\mathrm{L}$.

Delphinium pubescens DC.

Eranthis hyemalis (L.) Salisb.

Ficaria verna Huds. subsp. calthifolia (Rchb.) Nyman

Ficaria verna Huds. subsp. fertilis (Lawalrée ex Laegaard) Stace

Ficaria verna Huds. subsp. ficariiformis (F.W.Schultz) B.Walln.

Ficaria verna Huds. subsp. verna

Helleborus dumetorum Waldst. \& Kit. ex Willd. subsp. illyricus Starmühler 
Helleborus foetidus $L$. subsp. foetidus

Helleborus lividus Aiton subsp. corsicus (Briq.) P.Fourn.

Helleborus niger L. subsp. macranthus (Freyn) Schiffn.

Helleborus niger L. subsp. niger

T Helleborus odorus Waldst. \& Kit. subsp. laxus (Host) Merxm. \& Podlech

ET Helleborus viridis L. subsp. bocconei (Ten.) Peruzzi

ET Helleborus viridis L. subsp. abruzzicus (M.Thomsen, McLewin \& B.Mathew) Bartolucci, F.Conti \& Peruzzi

$\mathrm{T} \quad$ Helleborus viridis $\mathrm{L}$. subsp. istriacus (Schiffn.) Cristof. \& Zanotti

ET Helleborus viridis L. subsp. liguricus (M.Thomsen, McLewin \& B.Mathew) Bartolucci, F.Conti \& Peruzzi

Helleborus viridis $L$. subsp. viridis

Hepatica nobilis Mill.

Isopyrum thalictroides $\mathrm{L}$.

Myosurus minimus L.

Nigella arvensis $L$. subsp. arvensis

E Nigella arvensis L. subsp. glaucescens (Guss.) Greuter \& Burdet

\section{Nigella damascena $\mathrm{L}$.}

Nigella papillosa G.López subsp. atlantica (Murb.) Amich ex G.López

Pulsatilla alpina (L.) Delarbre subsp. alpina

Pulsatilla alpina (L.) Delarbre subsp. apiifolia (Scop.) Nyman

T Pulsatilla alpina (L.) Delarbre subsp. austriaca Aichele \& Schwegler

Pulsatilla alpina (L.) Delarbre subsp. austroalpina D.M.Moser

Pulsatilla alpina (L.) Delarbre subsp. cottianaea (Beauverd) D.M.Moser

Pulsatilla alpina (L.) Delarbre subsp. millefoliata (Bertol.) D.M.Moser

Pulsatilla halleri (All.) Willd. subsp. halleri

Pulsatilla montana (Hoppe) Rchb. subsp. montana

Pulsatilla vernalis (L.) Mill.

E Ranunculus abbaianus Dunkel

Ranunculus aconitifolius $\mathrm{L}$.

Ranunculus acris $L$. subsp. acris

Ranunculus acris $L$. subsp. friesianus (Jord.) Syme

Ranunculus aduncus Gren. \& Godr.

Ranunculus allemannii Braun-Blanq.

Ranunculus alpestris $\mathrm{L}$.

ET Ranunculus angulatus C.Presl

E Ranunculus apenninus (Chiov.) Pignatti

Ranunculus arvensis $L$.

E Ranunculus baldensis Dunkel

E Ranunculus bariscianus Dunkel

Ranunculus batrachioides Pomel

Ranunculus baudotii Godr.

E Ranunculus bilobus Bertol.

Ranunculus boreoapenninus Pignatti

E Ranunculus bovioi Dunkel

E Ranunculus braun-blanquetii Pignatti

Ranunculus brevifolius Ten.

Ranunculus breyninus Crantz

Ranunculus brutius Ten.

Ranunculus bulbosus $\mathrm{L}$.

Ranunculus bullatus $\mathrm{L}$.

Ranunculus carinthiacus Hoppe

Ranunculus cassubicifolius W.Koch

Ranunculus chius DC.

Ranunculus circinatus Sibth

E Ranunculus cochlearifer Dunkel

E Ranunculus cordiger Viv. subsp. cordiger

E Ranunculus cordiger Viv. subsp. diffusus (Moris) Arrigoni

E Ranunculus cymbalariifolius Balb. ex Moris

E Ranunculus farraensis Dunkel \& Poldini

E Ranunculus fiorii Pignatti - Note: Possibly extinct (Dunkel 2010).

Ranunculus flammula $\mathrm{L}$.

Ranunculus fluitans Lam.

Ranunculus fontanus C.Presl

E Ranunculus fraelensis Dunkel - Note: Possibly extinct (Dunkel 2010).

Ranunculus garganicus Ten.

E Ranunculus giordanoi F.Conti \& Bartolucci - Note: For the taxonomy and distribution of this species, see Conti and Bartolucci (2017); Conti et al. (2018a).

Ranunculus glacialis $\mathrm{L}$.

E Ranunculus gortanii Pignatti

Ranunculus gracilis E.D.Clarke

Ranunculus gramineus L.

E Ranunculus hostiliensis Pignatti - Note: Extinct (Dunkel 2010).

Ranunculus hybridus Biria

Ranunculus illyricus $\mathrm{L}$.

Ranunculus isthmicus Boiss.

Ranunculus kuepferi Greuter \& Burdet subsp. kuepferi

Ranunculus kuepferi Greuter \& Burdet subsp. orientalis W.Huber

Ranunculus lanuginosus L.

Ranunculus lateriflorus DC. 
Ranunculus lingua $\mathrm{L}$.

E Ranunculus luminarius Rigo ex Pignatti

Ranunculus macrophyllus Desf.

E Ranunculus magellensis Ten.

Ranunculus marginatus d'Urv.

E Ranunculus marsicus Guss. \& Ten.

E Ranunculus mediogracilis Dunkel

Ranunculus melzeri Hörandl \& Guterm.

Ranunculus millefoliatus Vahl

E Ranunculus monspeliacus L. subsp. aspromontanus (Huter) Peruzzi \& N.G.Passal.

Ranunculus monspeliacus $L$. subsp. monspeliacus

Ranunculus monspeliacus L. subsp. saxatilis (Balb.) Nyman - Note: Possibly extinct in Italy.

Ranunculus montanus Willd.

E Ranunculus multidens Dunkel

Ranunculus muricatus L.

E Ranunculus mutinensis Pignatti - Note: Extinct (Dunkel 2010).

Ranunculus neapolitanus Ten.

Ranunculus omiophyllus Ten.

Ranunculus ophioglossifolius Vill.

E Ranunculus palaeoeuganeus Pignatti

Ranunculus paludosus Poir.

Ranunculus parnassifolius $L$. subsp. heterocarpus P.Küpfer

Ranunculus parviflorus $\mathrm{L}$.

E Ranunculus pedemontanus Dunkel

E Ranunculus pedrottii Spinosi ex Dunkel

Ranunculus peltatus Schrank

Ranunculus penicillatus (Dumort.) Bab.

Ranunculus platanifolius $\mathrm{L}$.

E Ranunculus plavensis Dunkel

E Ranunculus poldinii Dunkel

E Ranunculus pollinensis (N.Terracc.) Chiov.

Ranunculus polyanthemoides Boreau

Ranunculus polyanthemophyllus W.Koch \& H.E.Hess

E Ranunculus pratensis C.Presl

E Ranunculus prosseri Dunkel

Ranunculus pseudofluitans (Syme) Newbould ex Beker \& Foggitt

Ranunculus pygmaeus Wahlenb.

Ranunculus repens $\mathrm{L}$.

Ranunculus reptans $\mathrm{L}$.

Ranunculus revelierei Boreau

Ranunculus rionii Lagger

Ranunculus saniculifolius Viv.

Ranunculus sardous Crantz

Ranunculus sceleratus $\mathrm{L}$.

Ranunculus seguieri Vill. subsp. seguieri

Ranunculus serbicus Vis.

Ranunculus serpens Schrank

E Ranunculus silanus Pignatti

E Ranunculus spicatus Desf. subsp. rupestris (Guss.) Maire

E Ranunculus thomasii Ten.

Ranunculus thora $\mathrm{L}$.

Ranunculus traunfellneri Hoppe

Ranunculus trichophyllus Chaix - Note: Ranunculus eradicatus (Laest.) Johans. [= R. confervoides (Fr.) Fr.] has to be excluded from Italy (Wiegleb et al. 2017), and probably its records from Alps belong to forms of the polymorphic $R$. trichophyllus. The name Batrachium eradicatum (Laest.) Fr., establishing the priority of the specific epithet at the species rank, is a combination validly published according to the Arts. 30.18 and 41.3 of the ICN (McNeill et al. 2012).

Ranunculus trilobus Desf.

Ranunculus tuberosus Lapeyr.

Ranunculus variabilis Hörandl \& Guterm.

Ranunculus velutinus Ten.

E Ranunculus venetus Huter ex Landolt

Ranunculus villarsii DC.

Staphisagria macrosperma Spach

Staphisagria requienii (Dc.) Spach subsp. picta (Willd.) Peruzzi

Staphisagria requienii (DC.) Spach subsp. requienii

Thalictrum alpinum $\mathrm{L}$.

Thalictrum aquilegiifolium L. subsp. aquilegiifolium

E Thalictrum calabricum Spreng.

Thalictrum flavum $\mathrm{L}$.

Thalictrum foetidum $L$. subsp. foetidum

Thalictrum lucidum $\mathrm{L}$.

Thalictrum minus $L$. subsp. minus

Thalictrum minus L. subsp. pratense (F.W.Schultz) Hand

Thalictrum minus $L$. subsp. saxatile Ces.

T Thalictrum simplex L. subsp. galioides (DC.) Korsh.

Thalictrum simplex L. subsp. simplex

Thalictrum simplex L. subsp. tenuifolium (Sw. ex Hartm.) Sterner

Trollius europaeus $\mathrm{L}$. 
Papaveraceae

Chelidonium majus $\mathrm{L}$.

Corydalis capnoides (L.) Pers.

Corydalis cava (L.) Schweigg. \& Körte subsp. cava

Corydalis densiflora C.Presl

Corydalis intermedia (L.) Mérat

Corydalis pumila (Host) Rchb.

Corydalis solida (L.) Clairv. subsp. solida

Fumaria agraria Lag.

Fumaria barnolae Sennen \& Pau subsp. barnolae

Fumaria bastardii Boreau

Fumaria bicolor Nicotra

Fumaria capreolata L. subsp. capreolata

Fumaria densiflora DC.

Fumaria flabellata Gasp.

Fumaria gaillardotii Boiss.

Fumaria judaica Boiss. subsp. judaica

Fumaria muralis W.D.J.Koch subsp. muralis

Fumaria nemorosa Lojac.

Fumaria officinalis $L$. subsp. officinalis

Fumaria officinalis L. subsp. wirtgenii (W.D.J.Koch) Arcang.

Fumaria parviflora Lam.

Fumaria petteri Rchb. subsp. petteri

Fumaria schleicheri Soy.-Will.

Fumaria vaillantii Loisel.

Glaucium corniculatum (L.) Rudolph subsp. corniculatum

Glaucium flavum Crantz

Hypecoum imberbe Sm.

Hypecoum procumbens $L$. subsp. procumbens

Hypecoum torulosum Å.E.Dahl

Papaver alpinum L. subsp. alpinum

Papaver apulum Ten.

C Papaver argemone L. subsp. argemone

Papaver dubium $\mathrm{L}$.

Papaver hybridum $\mathrm{L}$.

Papaver pinnatifidum Moris

C Papaver rhoeas L. subsp. rhoeas

Papaver setigerum DC.

Platycapnos spicatus (L.) Bernh.

Pseudofumaria alba (Mill.) Lidén subsp. alba

Pseudofumaria lutea (L.) Borkh.

Buxaceae

Buxus balearica Lam.

Buxus sempervirens $\mathrm{L}$.

Paeoniaceae

Paeonia coriacea Boiss.

E Paeonia corsica Sieber ex Tausch

Paeonia mascula (L.) Mill. subsp. mascula

ET Paeonia mascula (L.) Mill. subsp. russoi (Biv.) Cullen \& Heywood

E Paeonia morisii Cesca, Bernardo \& N.G.Passal.

Paeonia officinalis L. subsp. arietina (G.Anderson) N.G.Passal.

Paeonia officinalis L. subsp. banatica (Rochel) Soó

Paeonia officinalis L. subsp. huthii Soldano

E Paeonia officinalis L. subsp. italica N.G.Passal. \& Bernardo

Paeonia officinalis $\mathrm{L}$. subsp. officinalis

Paeonia peregrina Mill.

ET Paeonia sandrae Camarda

Crassulaceae

Bulliarda vaillantii (Willd.) DC

C Crassula alata (Viv.) A.Berger

Crassula tillaea Lest.-Garl.

Hylotelephium anacampseros (L.) H.Ohba

Hylotelephium maximum (L.) Holub subsp. maximum

Jovibarba allionii (Jord. \& Fourr.) D.A.Webb

Jovibarba arenaria (W.D.J.Koch) Opiz

Jovibarba globifera (L.) J.Parn. subsp. hirta (L.) J.Parn.

E Jovibarba globifera (L.) J.Parn. subsp. Iagariniana L.Gallo

Petrosedum amplexicaule (DC.) Velayos

Petrosedum montanum (Songeon \& E.P.Perrier) Grulich

E Petrosedum ochroleucum (Chaix) Niederle subsp. mediterraneum (L.Gallo) Niederle - Note: The populations from SIC deserve further studies.

Petrosedum ochroleucum (Chaix) Niederle subsp. ochroleucum

Petrosedum rupestre (L.) P.V.Heath

Petrosedum sediforme (Jacq.) Grulich

Petrosedum tenuifolium (Sm.) Grulich

Petrosedum thartii (L.P.Hébert) Niederle 
Phedimus stellatus (L.) Raf.

\section{Rhodiola rosea $\mathrm{L}$.}

Sedum acre L.

Sedum aetnense Tineo

Sedum album L. subsp. album

Sedum album L. subsp. micranthum (Bast. ex DC.) Syme

Sedum alpestre Vill.

E Sedum alsinifolium All.

Sedum andegavense (DC.) Desv.

Sedum annuum $\mathrm{L}$.

E Sedum aquilanum L.Gallo \& F.Conti - Note: For the taxonomy and distribution of this species, see Gallo and Conti (2015).

Sedum atratum $\mathrm{L}$.

Sedum brevifolium DC

Sedum caeruleum $\mathrm{L}$.

Sedum caespitosum (Cav.) DC.

Sedum cepaea $\mathrm{L}$.

Sedum dasyphyllum $\mathrm{L}$. subsp. dasyphyllum

Sedum dasyphyllum L. subsp. glanduliferum (Guss.) Nyman

Sedum fragrans 't Hart

Sedum glandulosum Moris - Note: The taxonomic identity of the population from TOS is doubtful and needs further studies.

Sedum gypsicola Boiss. \& Reut.

Sedum hirsutum All. subsp. hirsutum

Sedum hispanicum $\mathrm{L}$.

Sedum litoreum Guss.

E Sedum magellense Ten. subsp. magellense

Sedum magellense Ten. subsp. olympicum (Boiss.) Grande

Sedum monregalense Balb.

Sedum rubens $\mathrm{L}$.

Sedum sexangulare $\mathrm{L}$.

Sedum villosum $\mathrm{L}$.

Sempervivum alpinum Griseb. \& Schenk - Note: Sempervivum soculense D.Donati \& G.Dumont is here provisionally included.

Sempervivum arachnoideum $\mathrm{L}$.

C Sempervivum calcareum Jord.

E Sempervivum dolomiticum Facchini

Sempervivum glaucum Ten.

Sempervivum grandiflorum Haw.

Sempervivum montanum L. subsp. burnatii Wettst. ex Hayek

Sempervivum montanum L. subsp. montanum

E Sempervivum riccii lberite \& Anzal.

Sempervivum tectorum L. - Note: Sempervivum tectorum is native to Italy but cultivated since 2000 years or more, especially in Northern Italy. In the

North-Central Apennines it likely cohabits with S. alpinum Griseb. \& Schenk.

Sempervivum wulfenii Hoppe ex Mert. \& W.D.J.Koch subsp. wulfenii

C Umbilicus chloranthus Heldr. \& Sartori ex Boiss.

Umbilicus horizontalis (Guss.) DC. - Note: Some populations from SIC deserve further studies because of variability in flower morphology.

Umbilicus rupestris (Salisb.) Dandy

Haloragaceae

Myriophyllum alterniflorum DC.

Myriophyllum spicatum L.

Myriophyllum verticillatum $\mathrm{L}$.

Grossulariaceae

Ribes alpinum $\mathrm{L}$.

Ribes multiflorum Kit. ex Roem. \& Schult. subsp. multiflorum

E Ribes multiflorum Kit. ex Roem. \& Schult. subsp. sandalioticum Arrigoni

Ribes petraeum Wulfen

Ribes rubrum L. - Note: The native R. rubrum s.str. and the alien R. spicatum E.Robson are both included here.

E Ribes sardoum Martelli

Ribes uva-crispa L. subsp. austro-europeum Bornm.

Ribes uva-crispa L. subsp. uva-crispa

Saxifragaceae

Chrysosplenium alternifolium $\mathrm{L}$.

Chrysosplenium dubium Gay ex Ser.

Micranthes engleri (Dalla Torre) Galasso, Banfi \& Soldano

Saxifraga adscendens $L$. subsp. adscendens

Saxifraga adscendens L. subsp. parnassica (Boiss. \& Heldr.) Hayek

Saxifraga aizoides $L$.

Saxifraga androsacea $\mathrm{L}$.

Saxifraga aphylla Sternb.

E Saxifraga arachnoidea Sternb.

Saxifraga aspera $\mathrm{L}$.

E Saxifraga berica (Bég.) D.A.Webb

Saxifraga biflora All. subsp. biflora

Saxifraga bryoides L.

Saxifraga bulbifera $\mathrm{L}$.

Saxifraga burseriana $\mathrm{L}$.

Saxifraga caesia L. 
Saxifraga caprariae Mannocci, Ferretti, Mazzoncini \& Viciani

Saxifraga carpetana Boiss. \& Reut. subsp. graeca (Boiss. \& Heldr.) D.A.Webb

Saxifraga cernua $\mathrm{L}$.

Saxifraga cochlearis Rchb.

$E$

Sexifraga corsica (Ser. ex Duby) Gren. \& Godr. subsp. corsica

Saxifraga cotyledon $\mathrm{L}$.

Saxifraga crustata Vest

Saxifraga cuneifolia L. subsp. cuneifolia

T Saxifraga cuneifolia L. subsp. robusta D.A.Webb

E Saxifraga depressa Sternb.

Saxifraga diapensioides Bellardi

E Saxifraga exarata Vill. subsp. ampullacea (Ten.) D.A.Webb

Saxifraga exarata Vill. subsp. carniolica (Huter) T.Wraber

Saxifraga exarata Vill. subsp. exarata

Saxifraga exarata Vill. subsp. moschata (Wulfen) Cavill.

Saxifraga exarata Vill. subsp. pseudoexarata (Braun-Blanq.) D.A.Webb

Saxifraga facchinii W.D.J.Koch

Saxifraga florulenta Moretti

Saxifraga glabella Bertol.

Saxifraga granulata L. subsp. granulata

Saxifraga hederacea $\mathrm{L}$.

Saxifraga hirculus $\mathrm{L}$.

Saxifraga hostii Tausch subsp. hostii

E Saxifraga hostii Tausch subsp. rhaetica (A.Kern. ex Engl.) Braun-Blanq.

E Saxifraga italica D.A.Webb

Saxifraga marginata Sternb.

E Saxifraga montis-christi Mannocci, Ferretti, Mazzoncini \& Viciani

Saxifraga muscoides All.

Saxifraga mutata L. subsp. mutata

Saxifraga oppositifolia L. subsp. glandulifera Vacc

Saxifraga oppositifolia L. subsp. oppositifolia

T Saxifraga oppositifolia L. subsp. rudolphiana (Hornsch. ex W.D.J.Koch) Nyman

ET Saxifraga oppositifolia L. subsp. speciosa (Dörfl. \& Hayek) Engl. \& Irmsch.

Saxifraga paniculata Mill.

E Saxifraga pedemontana All. subsp. cervicornis (Viv.) Engl.

Saxifraga pedemontana All. subsp. pedemontana

Saxifraga petraea $\mathrm{L}$.

E Saxifraga porophylla Bertol. subsp. porophylla

E Saxifraga presolanensis Engl.

Saxifraga retusa Gouan subsp. augustana (Vacc.) D.A.Webb

Saxifraga retusa Gouan subsp. retusa

Saxifraga rotundifolia $L$. subsp. rotundifolia

Saxifraga sedoides $L$. subsp. sedoides

E Saxifraga sedoides L. subsp. tomentosa (Zenari) Poldini

Saxifraga seguieri Spreng.

Saxifraga squarrosa Sieber

Saxifraga taygetea Boiss. \& Heldr.

Saxifraga tenella Wulfen

E Saxifraga tombeanensis Boiss. ex Engl.

Saxifraga tridactylites $\mathrm{L}$.

Saxifraga valdensis DC.

Saxifraga vandellii Sternb.

Cynomoriaceae

Cynomorium coccineum L. subsp. coccineum

Vitaceae

Vitis vinifera $\mathrm{L}$.

Zygophyllaceae

Fagonia cretica L.

Tribulus terrestris $\mathrm{L}$.

Fabaceae

Colutea L. (Browicz 1963); Cytisus Desf. sect. Calicotome (Link) DC. (Cristofolini and Troì 2006); Lathyrus L., Ervilia Link, Ervum L., Lathyrus L., and Vicia L. (Schaefer et al. 2012); Trifolium sect. Vesicastrum subsect. Mystillus (C.Presl) Coulot \& Rebaute (Scoppola and Lattanzi 2016; Scoppola et al. 2016); Trigonella L. (Bena 2001).

E $\quad$ Adenocarpus complicatus (L.) J.Gay subsp. bivonae (C.Presl) Peruzzi

E Adenocarpus complicatus (L.) J.Gay subsp. brutius (Brullo, De Marco \& Siracusa) Peruzzi \& Bernardo

E Adenocarpus complicatus (L.) J.Gay subsp. commutatus (Guss.) Cout.

E Adenocarpus complicatus (L.) J.Gay subsp. samniticus (Brullo, De Marco \& Siracusa) Peruzzi

E Adenocarpus complicatus (L.) J.Gay subsp. tenoreanus (Brullo, Gangale \& Uzunov) Peruzzi \& Bernardo

Anagyris foetida L.

Anthyllis barba-jovis L.

E Anthyllis hermanniae L. subsp. brutia Brullo \& Giusso

ET Anthyllis hermanniae L. subsp. corsica Brullo \& Giusso - Note: Possibly extinct in Italy. 
E Anthyllis hermanniae L. subsp. ichnusae Brullo \& Giusso

Anthyllis hermanniae L. subsp. japygica Brullo \& Giusso

Anthyllis hermanniae L. subsp. sicula Brullo \& Giusso - Note: Possibly extinct.

Anthyllis montana L. subsp. jacquinii (A.Kern.) Hayek

Anthyllis montana $\mathrm{L}$. subsp. montana

Anthyllis vulneraria L. subsp. alpicola (Brügger) Gutermann

$\mathrm{T} \quad$ Anthyllis vulneraria L. subsp. baldensis (A.Kern. ex Sagorski) Pignatti ex Kerguélen

ET Anthyllis vulneraria L. subsp. busambarensis (Lojac.) Pignatti

Anthyllis vulneraria L. subsp. carpatica (Pant.) Nyman

Anthyllis vulneraria L. subsp. maura (Beck) Maire

Anthyllis vulneraria $L$. subsp. nana (Ten.) Tammaro

Anthyllis vulneraria L. subsp. pulchella (Vis.) Bornm.

Anthyllis vulneraria L. subsp. polyphylla (DC.) Nyman

Anthyllis vulneraria L. subsp. rubriflora (DC.) Arcang.

Anthyllis vulneraria L. subsp. sampaioana (Rothm.) Vasc.

Anthyllis vulneraria L. subsp. valesiaca (Beck) Guyot

Anthyllis vulneraria L. subsp. versicolor (Dalla Torre \& Sarnth.) Gutermann

Anthyllis vulneraria L. subsp. vulnerarioides (All.) Arcang. - Note: For the nomenclature and distribution of this subspecies, see Conti et al. (2016).

Anthyllis vulneraria L. subsp. weldeniana (Rchb.) Cullen

Argyrolobium zanonii (Turra) P.W.Ball subsp. zanonii

Astragalus alopecurus Pall.

Astragalus alpinus $\mathrm{L}$.

E Astragalus aquilanus Anzal.

Astragalus australis (L.) Lam.

Astragalus austriacus Jacq.

Astragalus boeticus L.

E Astragalus caprinus L. subsp. huetii (Bunge) Podlech

Astragalus cicer L.

Astragalus danicus Retz.

Astragalus depressus L. subsp. depressus

Astragalus echinatus Murray

Astragalus epiglottis L. subsp. epiglottis

Astragalus exscapus L. subsp. exscapus

Astragalus frigidus (L.) A.Gray

$\mathrm{E} \quad$ Astragalus genargenteus Moris

E Astragalus gennarii Bacch. \& Brullo

Astragalus glycyphyllos L.

Astragalus hamosus L.

Astragalus hypoglottis L. subsp. gremlii (Burnat) Greuter \& Burdet

Astragalus hypoglottis L. subsp. hypoglottis

E Astragalus kamarinensis C.Brullo, Brullo, Giusso, Miniss. \& Sciandr.

Astragalus leontinus Wulfen

E Astragalus maritimus Moris

Astragalus monspessulanus L. subsp. monspessulanus

Astragalus monspessulanus L. subsp. wulfenii (W.D.J.Koch) Arcang.

E Astragalus muelleri Steud. \& Hochst. subsp. etruscus Peruzzi, Gestri \& Pierini - Note: For the taxonomy and distribution of this subspecies, see Peruzzi et al.

(2014c).

E Astragalus nebrodensis (Guss.) Strobl

Astragalus onobrychis L.

E Astragalus parnassi Boiss. subsp. calabricus (Fisch.) Maassoumi

Astragalus pastellianus Pollini

Astragalus pelecinus (L.) Barneby subsp. pelecinus

Astragalus penduliflorus Lam.

Astragalus peregrinus Vahl subsp. warionis (Gand.) Maire

E Astragalus raphaelis Ferro

Astragalus scorpioides Willd.

Astragalus sempervirens Lam.

Astragalus sesameus L.

E Astragalus siculus Biv.

Astragalus sirinicus Ten.

Astragalus tegulensis Bacch. \& Brullo

Astragalus terraccianoi Vals.

Astragalus thermensis Vals.

Astragalus verrucosus Moris

Astragalus vesicarius L. subsp. carniolicus (A.Kern.) Chater

Astragalus vesicarius $L$. subsp. vesicarius

ET Bituminaria basaltica Miniss., C.Brullo, Brullo, Giusso \& Sciandr.

Bituminaria bituminosa (L.) C.H.Stirt.

E Bituminaria morisiana (Pignatti \& Metlesics) Greuter

Cercis siliquastrum L. subsp. siliquastrum

Colutea arborescens L.

$\mathrm{T} \quad$ Colutea brevialata Lange

Coronilla coronata $\mathrm{L}$.

Coronilla juncea $\mathrm{L}$.

Coronilla minima L. subsp. minima

Coronilla repanda (Poir.) Guss. subsp. repanda

Coronilla scorpioides (L.) W.D.J.Koch

Coronilla vaginalis Lam. 
Coronilla valentina $\mathrm{L}$.

Cytisophyllum sessilifolium (L.) O.Lang

Cytisus aeolicus Guss.

Cytisus decumbens (Durande) Spach

Cytisus emeriflorus Rchb.

Cytisus hirsutus L.

Cytisus infestus (C.Presl) Guss. subsp. infestus

Cytisus infestus (C.Presl) Guss. subsp. intermedius (Salzm. ex C.Presl) Cristof. \& Trò̀

Cytisus laniger DC.

Cytisus nigricans L. subsp. atratus (Schur) Nyman

Cytisus nigricans L. subsp. nigricans

Cytisus proteus Zumagl.

Cytisus pseudoprocumbens Markgr.

Cytisus purpureus Scop.

Cytisus scoparius (L.) Link subsp. scoparius

Cytisus spinescens C.Presl

Cytisus spinosus (L.) Lam.

Cytisus villosus Pourr.

Dorycnopsis gerardi (L.) Boiss.

T Emerus major Mill. subsp. emeroides (Boiss. \& Spruner) Soldano \& F.Conti

Emerus major Mill. subsp. major

Ervilia articulata (Hornem.) H.Schaef.

Ervilia hirsuta (L.) Opiz

Ervilia Ioiseleurii (M.Bieb.) H.Schaef.

Ervilia sylvatica (L.) Schur

Ervum gracile DC.

Ervum pubescens DC.

Ervum tetraspermum $\mathrm{L}$.

Genista anglica $\mathrm{L}$.

E Genista arbusensis Vals.

E Genista aristata C.Presl

Genista aspalathoides Lam.

E Genista bocchierii Bacch., Brullo \& Feoli Chiapella

E Genista cadasonensis Vals.

E Genista cilentina Vals.

Genista cinerea (Vill.) DC

E Genista corsica (Loisel.) DC

Genista cupanii Guss.

Genista demarcoi Brullo, Scelsi \& Siracusa

Genista desoleana Vals.

Genista ephedroides DC.

Genista etnensis (Raf.) DC.

Genista ferox (Poir.) Poir.

E Genista gasparrinii (Guss.) C.Presl

Genista germanica $\mathrm{L}$.

Genista hispanica L. subsp. hispanica

Genista holopetala (Koch) Bald.

E Genista insularis Bacch., Brullo \& Feoli Chiapella subsp. fodinae Bacch., Brullo \& Feoli Chiapella

Genista insularis Bacch., Brullo \& Feoli Chiapella subsp. insularis

Genista januensis Viv. subsp. januensis

C Genista linifolia L.

E Genista madoniensis Raimondo

E Genista michelii Spach

Genista monspessulana (L.) L.A.S.Johnson

E Genista morisii Colla

E Genista ovina Bacch., Brullo \& Feoli Chiapella

E Genista pichisermolliana Vals.

Genista pilosa L.

E Genista pulchella Vis. subsp. aquilana F.Conti \& Manzi

Genista radiata (L.) Scop.

Genista sagittalis L.

E Genista salzmannii DC

E Genista sardoa Vals.

E Genista sericea Wulfen subsp. pollinensis F.Conti, Feoli Chiapella \& Bernardo

Genista sericea Wulfen subsp. sericea - Note: For the taxonomy and distribution of this species, see Conti et al. (2014).

E Genista sulcitana Vals.

Genista sylvestris Scop.

ET Genista tenorei G.Don

Genista tinctoria L.

E Genista toluensis Vals.

E Genista tyrrhena Vals. subsp. pontiana Brullo \& De Marco

Genista tyrrhena Vals. subsp. tyrrhena

Genista valsecchiae Brullo \& De Marco

Glycyrrhiza glabra L.

Hedysarum brigantiacum Bourn., Chas \& Kerguélen

Hedysarum confertum Desf.

Hedysarum hedysaroides (L.) Schinz \& Thell. subsp. exaltatum (A.Kern.) Chrtková

Hedysarum hedysaroides (L.) Schinz \& Thell. subsp. hedysaroides 
Hippocrepis biflora Spreng.

Hippocrepis ciliata Willd.

Hippocrepis comosa L. subsp. comosa

Hippocrepis glauca Ten.

Hippocrepis multisiliquosa $\mathrm{L}$.

Hymenocarpos circinnatus (L.) Savi

Laburnum alpinum (Mill.) Bercht. \& J.Pres|

Laburnum anagyroides Medik. subsp. alschingeri (Vis.) Hayek

Laburnum anagyroides Medik. subsp. anagyroides

Lathyrus amphicarpos $\mathrm{L}$.

Lathyrus angulatus $\mathrm{L}$.

Lathyrus annuus $L$.

E Lathyrus apenninus F.Conti - Note: For the taxonomy and distribution of this species, see Conti (2010).

Lathyrus aphaca L. subsp. aphaca

Lathyrus cicera $\mathrm{L}$.

Lathyrus cirrhosus Ser.

Lathyrus clymenum $\mathrm{L}$.

Lathyrus digitatus (M.Bieb.) Fiori

Lathyrus filiformis (Lam.) J.Gay

Lathyrus gorgoni Parl.

Lathyrus grandiflorus Sm.

Lathyrus heterophyllus $\mathrm{L}$.

Lathyrus hirsutus $\mathrm{L}$.

Lathyrus inconspicuus L.

E Lathyrus jordanii (Ten.) Ces., Pass. \& Gibelli

Lathyrus laevigatus (Waldst. \& Kit.) Gren. subsp. laevigatus

Lathyrus laevigatus (Waldst. \& Kit.) Gren. subsp. occidentalis (Fisch. \& C.A.Mey.) Breistr.

Lathyrus latifolius $\mathrm{L}$.

Lathyrus Iaxiflorus (Desf.) Kuntze subsp. Iaxiflorus

Lathyrus linifolius (Reichard) Bässler

Lathyrus niger (L.) Bernh.

Lathyrus nissolia $\mathrm{L}$.

Lathyrus ochrus (L.) DC.

E Lathyrus odoratus $L$.

Lathyrus oleraceus Lam. subsp. biflorus (Raf.) H.Schaef., Coulot \& Rabaute

Lathyrus palustris $\mathrm{L}$.

Lathyrus pannonicus (Jacq.) Garcke subsp. asphodeloides (Gouan) Bässler

Lathyrus pannonicus (Jacq.) Garcke subsp. varius (Hill) P.W.Ball

Lathyrus pratensis L. subsp. Iusseri (Heer ex W.D.J.Koch) Soják

Lathyrus pratensis $L$. subsp. pratensis

Lathyrus setifolius $\mathrm{L}$.

Lathyrus sphaericus Retz.

Lathyrus sylvestris L. subsp. sylvestris

Lathyrus tingitanus $\mathrm{L}$.

Lathyrus tuberosus L.

Lathyrus venetus (Mill.) Wohlf.

Lathyrus vernus (L.) Bernh.

Lotus angustissimus $\mathrm{L}$.

Lotus biflorus Desr.

Lotus conimbricensis Brot.

Lotus conjugatus $L$. subsp. conjugatus

Lotus conjugatus L. subsp. requienii (Mauri ex Sanguin.) Greuter

Lotus corniculatus $\mathrm{L}$. subsp. alpinus (DC.) Rothm.

Lotus corniculatus $L$. subsp. corniculatus

T Lotus corniculatus $L$. subsp. delortii (Timb.-Lagr.) Nyman

Lotus corniculatus L. subsp. preslii (Ten.) P.Fourn.

Lotus creticus $\mathrm{L}$.

Lotus cytisoides $\mathrm{L}$.

Lotus dorycnium $\mathrm{L}$.

Lotus edulis L.

Lotus germanicus (Gremli) Peruzzi

Lotus halophilus Boiss. \& Spruner

Lotus herbaceus (Vill.) Jauzein

Lotus hirsutus $\mathrm{L}$.

Lotus hispidus DC.

Lotus longisiliquosus R.Roem.

Lotus maritimus L.

Lotus ornithopodioides $L$.

Lotus parviflorus Desf.

Lotus pedunculatus Cav.

Lotus peregrinus $L$.

Lotus rectus $\mathrm{L}$.

Lotus tenuis Waldst. \& Kit. ex Willd.

Lotus tetragonolobus $\mathrm{L}$.

Lupinus albus L. subsp. graecus (Boiss. \& Spruner) Franco \& P.Silva

Lupinus angustifolius $\mathrm{L}$.

Lupinus cosentinii Guss.

Lupinus gussoneanus J.Agardh 
Lupinus luteus L.

Medicago arabica (L.) Huds.

Medicago arborea $\mathrm{L}$.

Medicago carstiensis Wulfen

Medicago ciliaris (L.) All.

Medicago coronata (L.) Bartal.

Medicago disciformis DC.

Medicago doliata Carmign.

Medicago falcata $L$. subsp. falcata

Medicago glutinosa M.Bieb.

Medicago intertexta (L.) Mill.

Medicago italica (Mill.) Grande

Medicago lesinsii E.Small

Medicago littoralis Loisel.

Medicago Iupulina $\mathrm{L}$.

Medicago marina $\mathrm{L}$.

Medicago minima (L.) L.

Medicago monspeliaca (L.) Trautv.

Medicago murex Willd.

Medicago muricoleptis Tineo

Medicago orbicularis (L.) Bartal.

Medicago pironae Vis.

Medicago polymorpha $\mathrm{L}$.

Medicago praecox DC.

Medicago prostrata Jacq. subsp. prostrata

Medicago rigidula (L.) All.

Medicago rugosa Desr.

Medicago scutellata (L.) Mill.

Medicago secundiflora Durieu

Medicago soleirolii Duby

Medicago tenoreana DC

Medicago truncatula Gaertn.

Medicago turbinata (L.) All.

Onobrychis aequidentata (Sm.) d'Urv.

Onobrychis alba (Waldst. \& Kit.) Desv. subsp. alba

Onobrychis alba (Waldst. \& Kit.) Desv. subsp. echinata (Guss.) P.W.Ball

Onobrychis alba (Waldst. \& Kit.) Desv. subsp. pentelica (Hausskn.) Nyman

Onobrychis arenaria (Kit.) DC. subsp. arenaria

Onobrychis arenaria (Kit.) DC. subsp. taurerica Hand.-Mazz.

Onobrychis arenaria (Kit.) DC. subsp. tommasinii (Jord.) Asch. \& Graebn.

ET Onobrychis calabrica Sirjaev

Onobrychis caput-galli (L.) Lam.

Onobrychis montana DC. subsp. montana

Onobrychis saxatilis (L.) Lam.

Onobrychis supina (Chaix ex Vill.) DC.

Onobrychis viciifolia Scop.

Ononis alba Poir. subsp. alba

Ononis alopecuroides L. subsp. exalopecuroides (G.López) Greuter \& Burdet

Ononis biflora Desf.

E Ononis cristata Mill. subsp. apennina Tammaro \& Catonica

Ononis cristata Mill. subsp. cristata

Ononis dentata Lowe

Ononis diffusa Ten.

Ononis hispida Desf. subsp. hispida

E Ononis masquillierii Bertol.

Ononis minutissima $\mathrm{L}$.

Ononis mitissima $\mathrm{L}$.

Ononis natrix L. subsp. hispanica (L.f.) Cout.

Ononis natrix L. subsp. natrix

Ononis natrix L. subsp. ramosissima (Desf.) Batt.

E Ononis oligophylla Ten.

Ononis ornithopodioides L.

Ononis pendula Desf. subsp. boissieri (Širj.) Devesa

Ononis pubescens $\mathrm{L}$.

Ononis pusilla $\mathrm{L}$. subsp. pusilla

Ononis reclinata $\mathrm{L}$.

Ononis rotundifolia $\mathrm{L}$.

Ononis serrata Forssk.

Ononis sicula Guss.

Ononis sieberi DC.

Ononis spinosa L. subsp. antiquorum (L.) Arcang.

Ononis spinosa L. subsp. arvensis (L.) Greuter \& Burdet

Ononis spinosa L. subsp. austriaca (Beck) Gams

Ononis spinosa L. subsp. procurrens (Wallr.) Briq.

Ononis spinosa $\mathrm{L}$. subsp. spinosa

Ononis variegata $L$.

Ononis viscosa L. subsp. breviflora (DC.) Nyman

Ornithopus compressus $\mathrm{L}$. 
Ornithopus perpusillus $\mathrm{L}$.

Ornithopus pinnatus (Mill.) Druce

Oxytropis campestris (L.) DC.

Oxytropis fetida (Vill.) DC.

Oxytropis halleri Bunge ex W.D.J.Koch

Oxytropis helvetica Scheele

Oxytropis lapponica (Wahlenb.) J.Gay

Oxytropis montana L.

Oxytropis neglecta J.Gay ex Ten.

E Oxytropis ocrensis F.Conti \& Bartolucci - Note: For the taxonomy and distribution of this species, see Conti et al. (2018b).

E Oxytropis pilosa (L.) DC. subsp. caputoi (Moraldo \& la Valva) Brilli-Catt., Di Massimo \& Gubellini

Oxytropis pilosa (L.) DC. subsp. pilosa

Oxytropis xerophila Gutermann

E Retama raetam (Forssk.) Webb \& Berthel. subsp. gussonei (Webb) Greuter

Scorpiurus muricatus $\mathrm{L}$.

Scorpiurus subvillosus $\mathrm{L}$.

DD Scorpiurus sulcatus $L$.

Scorpiurus vermiculatus $\mathrm{L}$.

Securigera cretica (L.) Lassen

Securigera securidaca (L.) Degen \& Dörfl.

Securigera varia $(\mathrm{L}$.) Lassen

Spartium junceum $L$.

Sulla capitata (Desf.) B.H.Choi \& H.Ohashi

Sulla coronaria (L.) Medik.

Sulla spinosissima (L.) B.H.Choi \& H.Ohashi

Trifolium alpestre $\mathrm{L}$.

Trifolium alpinum $\mathrm{L}$.

Trifolium angustifolium $L$. subsp. angustifolium

Trifolium arvense $L$. subsp. arvense

Trifolium arvense L. subsp. gracile (Thuill.) Nyman

Trifolium aureum Pollich subsp. aureum

Trifolium badium Schreb.

E Trifolium bivonae Guss.

Trifolium bocconei Savi - Note: For the typification of this name, see Roma-Marzio et al. (2018).

E Trifolium brutium Ten.

Trifolium campestre Schreb.

Trifolium cernuum Brot.

Trifolium cherleri $\mathrm{L}$.

Trifolium clusii Gren. \& Godr.

Trifolium congestum Guss.

Trifolium diffusum Ehrh.

Trifolium dubium Sibth.

Trifolium echinatum M.Bieb.

Trifolium fragiferum $L$. subsp. fragiferum

Trifolium glomeratum $\mathrm{L}$.

Trifolium grandiflorum Schreb.

Trifolium hirtum All.

Trifolium hybridum L. subsp. elegans (Savi) Asch. \& Graebn.

Trifolium hybridum L. subsp. hybridum

Trifolium incarnatum L. subsp. molinerii (Balb. ex Hornem.) Ces.

Trifolium infamia-ponertii Greuter

Trifolium isthmocarpum Brot. subsp. jaminianum (Boiss.) Murb.

Trifolium Iappaceum L.

Trifolium latinum Sebast. - Note: For the typification of this name, see lamonico et al. (2011).

Trifolium leucanthum M.Bieb.

Trifolium ligusticum Loisel.

Trifolium Iucanicum Gasp. ex Guss.

Trifolium medium L. subsp. medium

Trifolium michelianum Savi - Note: For the typification of this name, see Roma-Marzio et al. (2018).

Trifolium micranthum Viv.

Trifolium montanum L. subsp. montanum

Trifolium montanum L. subsp. rupestre (Ten.) Nyman

Trifolium multistriatum W.D.J.Koch

Trifolium mutabile Port.

Trifolium nigrescens Viv. subsp. nigrescens

Trifolium nigrescens Viv. subsp. petrisavii (Clementi) Holmboe

Trifolium noricum Wulfen subsp. noricum

Trifolium noricum Wulfen subsp. praetutianum (Savi) Arcang.

Trifolium obscurum Savi - Note: For the typification of this name, see Roma-Marzio et al. (2018).

Trifolium ochroleucon Huds.

Trifolium ornithopodioides $\mathrm{L}$.

Trifolium pallescens Schreb.

Trifolium pallidum Waldst. \& Kit.

Trifolium pannonicum Jacq. subsp. pannonicum

Trifolium patens Schreb.

Trifolium patulum Tausch

Trifolium phleoides Willd.

Trifolium physodes M.Bieb.

Trifolium pratense $\mathrm{L}$. subsp. nivale Ces. 
Trifolium pratense L. subsp. pratense

Trifolium pratense L. subsp. semipurpureum (Strobl) Pignatti

Trifolium purpureum Loisel.

Trifolium repens $\mathrm{L}$.

Trifolium resupinatum $\mathrm{L}$.

Trifolium rubens $\mathrm{L}$.

Trifolium saxatile All.

Trifolium scabrum L.

Trifolium sebastiani Savi - Note: For the typification of this name, see Roma-Marzio et al. (2018).

Trifolium spadiceum $\mathrm{L}$.

Trifolium spumosum $\mathrm{L}$.

Trifolium squamosum $\mathrm{L}$.

Trifolium squarrosum $\mathrm{L}$.

Trifolium stellatum $\mathrm{L}$.

Trifolium striatum L. subsp. striatum

Trifolium striatum L. subsp. tenuiflorum (Ten.) Arcang.

Trifolium strictum $\mathrm{L}$.

Trifolium subterraneum L. subsp. oxaloides Nyman

Trifolium subterraneum L. subsp. subterraneum

Trifolium subterraneum L. subsp. yanninicum Katzn. \& F.H.W.Morley

Trifolium suffocatum $\mathrm{L}$.

Trifolium sylvaticum Gérard ex Loisel.

Trifolium tenuifolium Ten.

Trifolium thalii Vill.

Trifolium tomentosum $\mathrm{L}$.

Trifolium uniflorum L. subsp. savianum (Guss.) Asch. \& Graebn.

Trifolium vesiculosum Savi - Note: For the typification of this name, see Roma-Marzio et al. (2018).

Trigonella alba (Medik.) Coulot \& Rabaute

Trigonella altissima (Thuill.) Coulot \& Rabaute

Trigonella elegans (Salzm. ex Ser.) Coulot \& Rabaute

Trigonella esculenta Willd.

Trigonella gladiata M.Bieb.

Trigonella infesta (Guss.) Coulot \& Rabaute

Trigonella italica (L.) Coulot \& Rabaute

Trigonella maritima Delile ex Poir.

Trigonella officinalis (L.) Coulot \& Rabaute

Trigonella segetalis (Brot.) Coulot \& Rabaute

Trigonella sicula (Turra) Coulot \& Rabaute

Trigonella smalii Coulot \& Rabaute

Trigonella sulcata (Desf.) Coulot \& Rabaute

Trigonella wojciechowskii Coulot \& Rabaute

Tripodion tetraphyllum (L.) Fourr.

Ulex europaeus L. subsp. europaeus

Vicia altissima Desf.

Vicia amphicarpa $\mathrm{L}$.

Vicia angustifolia $\mathrm{L}$

Vicia benghalensis L.

Vicia bithynica (L.) L.

Vicia cassubica $\mathrm{L}$.

E Vicia consentina Spreng.

Vicia cordata Wulfen ex Hoppe

Vicia cracca L.

Vicia cusnae Foggi \& Ricceri

Vicia dalmatica A.Kern

Vicia dasycarpa Ten.

Vicia disperma $\mathrm{DC}$.

Vicia dumetorum $\mathrm{L}$

Vicia eriocarpa (Hausskn.) Halácsy

Vicia ervoides (Brign.) Hampe

E

Vicia giacominiana Segelb.

Vicia glauca C.Presl subsp. glauca

Vicia grandiflora Scop.

Vicia hybrida L.

Vicia incana Gouan

Vicia incisa M.Bieb. - Note: For the taxonomy of this species, see lberite et al. (2017).

Vicia johannis Tamamsch.

Vicia laeta Ces.

Vicia lathyroides L.

Vicia leucantha Biv.

Vicia lutea $\mathrm{L}$.

Vicia macrocarpa (Moris) Bertol.

Vicia melanops Sm.

Vicia microphylla d'Urv.

Vicia monantha Retz. subsp. calcarata (Desf.) Romero Zarco

Vicia narbonensis $\mathrm{L}$.

Vicia nigricans (M.Bieb.) Coss. \& Germ.

E Vicia ochroleuca Ten. subsp. ochroleuca

Vicia onobrychioides L. 
Vicia oreophila Zertová

Vicia oroboides Wulfen

Vicia pannonica Crantz subsp. pannonica

Vicia pannonica Crantz subsp. striata (M.Bieb.) Nyman

Vicia peregrina $\mathrm{L}$.

Vicia pisiformis $\mathrm{L}$.

Vicia pseudocracca Bertol.

Vicia sativa $\mathrm{L}$.

Vicia saxatilis (Vent.) Tropea

Vicia segetalis Thuill.

Vicia sepium $\mathrm{L}$.

Vicia serinica R.Uechtr. \& Huter ex Porta

Vicia serratifolia Jacq.

Vicia sicula (Raf.) Guss.

Vicia sparsiflora Ten.

E Vicia tenuifolia Roth subsp. elegans (Guss.) Nyman

Vicia tenuifolia Roth subsp. tenuifolia

Vicia villosa Roth

Polygalaceae

Taxonomic references: Polygala L. (Arrigoni 2014).

Polygala alpestris Rchb. subsp. alpestris

ET Polygala alpestris Rchb. subsp. angelisii (Ten.) Nyman

ET Polygala alpestris Rchb. subsp. meridionalis Arrigoni

Polygala alpina (Poir. ex DC.) Steud.

T Polygala amara L. subsp. brachyptera (Chodat) Hayek

Polygala amarella Crantz

E Polygala apiculata Huter, Porta \& Rigo

Polygala calcarea F.W.Schultz

E Polygala carueliana (Burnat ex A.W.Benn.) Caruel

Polygala chamaebuxus $L$.

Polygala comosa Schkuhr

Polygala exilis DC.

E Polygala flavescens DC. subsp. flavescens - Note: For the typification of this name, see Roma-Marzio and Peruzzi (2017).

ET Polygala flavescens DC. subsp. maremmana (Fiori) Arrigoni

Polygala flavescens DC. subsp. pisaurensis (Caldesi) Arcang.

Polygala forojulensis A.Kern. subsp. adriatica (Chodat) Arrigoni

Polygala forojulensis A.Kern. subsp. carniolica (A.Kern.) Arrigoni

Polygala forojulensis A.Kern. subsp. forojulensis

Polygala major Jacq.

Polygala monspeliaca $\mathrm{L}$.

Polygala nicaeensis Risso ex W.D.J.Koch subsp. gariodiana (Jord. \& Fourr.) Chodat

ET Polygala nicaeensis Risso ex W.D.J.Koch subsp. italiana (Chodat) Arrigoni

T Polygala nicaeensis Risso ex W.D.J.Koch subsp. mediterranea Chodat

Polygala nicaeensis Risso ex W.D.J.Koch subsp. nicaeensis

ET Polygala nicaeensis Risso ex W.D.J.Koch subsp. peninsularis Arrigoni

E Polygala padulae Arrigoni

Polygala pedemontana E.P.Perrier \& B.Verl.

E Polygala preslii Spreng.

Polygala pseudoalpestris (Gren.) Dalla Torre \& Sarnth.

Polygala rupestris Pourr.

E Polygala sardoa Chodat

Polygala saxatilis Desf.

Polygala serpyllifolia Hosé

Polygala sinisica Arrigoni

T Polygala vulgaris L. subsp. oxyptera (Rchb.) Schübl. \& G.Martens

ET Polygala vulgaris L. subsp. valdarnensis (Fiori) Arrigoni

Polygala vulgaris $L$. subsp. vulgaris

Rosaceae

Taxonomic references: Alchemilla L. (Festi 2000; Tondi et al. 2005; Fröhner et al. 2012).

Agrimonia eupatoria L. subsp. eupatoria - Note: Agrimonia eupatoria L. subsp. grandis (Asch. \& Graebn.) Bornm. is included within the variability of A. eupatoria s.str. For the morphological variability of A. eupatoria, see lamonico (2017).

Agrimonia procera Wallr.

Alchemilla acrodon S.E.Fröhner

Alchemilla acutata Buser

Alchemilla aggregata Buser

Alchemilla alpigena Buser ex Hegi

Alchemilla alpina $\mathrm{L}$.

Alchemilla alpinula S.E.Fröhner

Alchemilla amphisericea Buser

E Alchemilla austroitalica Brullo, Scelsi \& Spamp.

E Alchemilla bonae S.E.Fröhner

Alchemilla carniolica (Paulin) Fritsch

Alchemilla cataractarum S.E.Fröhner

ET Alchemilla cavillieri (Briq.) Camus

ET Alchemilla ceroniana Buser 
Alchemilla chirophylla Buser

Alchemilla cinerea Buser - Note: Considering the records of related species ("pubescentes" group) from central Apennines, the distribution of $A$. cinerea in the Italian peninsula needs further investigations. Moreover, the taxonomic autonomy of this species is doubtful and it could represent just an extreme form of $A$. colorata Buser.

Alchemilla colorata Buser Alchemilla compta Buser

Alchemilla conjuncta Bab.

Alchemilla connivens Buser

Alchemilla coriacea Buser

Alchemilla crinita Buser

Alchemilla croatica Gand.

E Alchemilla cuneata Gaudin

Alchemilla cymatophylla Juz.

Alchemilla debilicaulis Buser

Alchemilla decumbens Buser

Alchemilla demissa Buser

ET Alchemilla diversiloba Buser

Alchemilla effusa Buser

Alchemilla exigua Buser ex Paulin

Alchemilla fallax Buser

E Alchemilla federiciana S.E.Fröhner

Alchemilla filicaulis Buser

Alchemilla firminiana Buser

Alchemilla fissa Günther \& Schummel

Alchemilla flabellata Buser

Alchemilla flavicoma Buser ex Schröt.

Alchemilla flavovirens Buser

Alchemilla frigens Buser ex Jaquet

Alchemilla glabra Neygenf.

Alchemilla glacialis Buser

Alchemilla glaucescens Wallr.

Alchemilla glomerulans Buser

Alchemilla grossidens Buser

Alchemilla helvetica Brügger

Alchemilla hirtipes Buser

Alchemilla hoppeana (Rchb.) Dalla Torre

Alchemilla impexa Buser

Alchemilla incisa Buser

Alchemilla inconcinna Buser

E Alchemilla lasenii S.E.Fröhner

Alchemilla leptoclada Buser

Alchemilla lineata Buser

Alchemilla longana Buser

Alchemilla longiuscula Buser

Alchemilla lucida Buser

Alchemilla lunaria S.E.Fröhner

E Alchemilla marsica Buser

E Alchemilla martinii S.E.Fröhner

Alchemilla micans Buser

Alchemilla monticola Opiz

E Alchemilla niphogeton Buser ex Pamp.

Alchemilla nitida Buser

E Alchemilla nydeggeriana S.E.Fröhner

Alchemilla obtusa Buser

Alchemilla opaca Buser

Alchemilla othmarii Buser

Alchemilla pallens Buser

Alchemilla pentaphyllea $\mathrm{L}$.

Alchemilla plicata Buser

Alchemilla propinqua H.Lindb. ex Juz.

Alchemilla radiisecta Buser

Alchemilla reniformis Buser

Alchemilla rhododendrophila Buser

Alchemilla rubristipula Buser

Alchemilla saxatilis Buser

Alchemilla sericoneura Buser

Alchemilla sinuata Buser

Alchemilla straminea Buser

Alchemilla strigosula Buser

Alchemilla subcrenata Buser

Alchemilla subglobosa C.G.Westerl.

Alchemilla subsericea Reut.

Alchemilla tenerrima S.E.Fröhner

Alchemilla tenuis Buser

Alchemilla tirolensis Buser ex Dalla Torre \& Sarnth.

Alchemilla transiens (Buser) Buser

Alchemilla trunciloba Buser

Alchemilla undulata Buser 
Alchemilla vaccariana Buser

Alchemilla venosula Buser

Alchemilla versipila Buser

Alchemilla vetteri Buser

Alchemilla vulgaris $\mathrm{L}$.

Alchemilla xanthochlora Rothm.

Amelanchier ovalis Medik. subsp. cretica (Willd.) Maire \& Petitm.

Amelanchier ovalis Medik. subsp. embergeri Favarger \& Stearn

Amelanchier ovalis Medik. subsp. ovalis

Aphanes arvensis $\mathrm{L}$.

Aphanes australis Rydb.

Aphanes floribunda (Murb.) Rothm.

Aphanes minutiflora (Azn.) Holub

Aphanes pusilla (Pomel) Batt.

Aremonia agrimonoides (L.) DC. subsp. agrimonoides

Argentina anserina (L.) Rydb. subsp. anserina

Aruncus dioicus (Walter) Fernald

Comarum palustre $\mathrm{L}$.

DD Cotoneaster favargeri J.Fryer \& B.Hylmö

Cotoneaster intermedius (Lecoq \& Lamotte) H.J.Coste

DD Cotoneaster juranus Gand.

E Cotoneaster nebrodensis (Guss.) K.Koch

Cotoneaster pyrenaicus Gand.

Cotoneaster tomentosus (Aiton) Lindl.

E Crataegus insegnae (Tineo) Bertol.

Crataegus laciniata Ucria

Crataegus laevigata (Poir.) DC.

DD Crataegus macrocarpa Hegetschw.

Crataegus monogyna Jacq.

Crataegus orientalis M.Bieb. subsp. orientalis

Crataegus orientalis M.Bieb. subsp. presliana K.I.Chr.

Dasiphora fruticosa (L.) Rydb.

Dryas octopetala L. subsp. octopetala

E Drymocallis corsica (Soleirol ex Lehm.) Kurtto

Drymocallis rupestris (L.) Soják

Filipendula ulmaria (L.) Maxim.

Filipendula vulgaris Moench

Fragaria moschata Weston

Fragaria vesca $L$. subsp. vesca

Fragaria viridis Weston subsp. viridis

Geum heterocarpum Boiss. - Note: For the taxonomy and distribution of this species, see Bartolucci and Conti (2013).

Geum molle Vis. \& Pančić

Geum montanum L.

Geum reptans L.

Geum rivale L.

Geum sylvaticum Pourr.

Geum urbanum L.

E Malus crescimannoi Raimondo

Malus florentina (Zuccagni) C.K.Schneid.

Malus sylvestris (L.) Mill.

Potentilla alba $\mathrm{L}$.

Potentilla alpicola De la Soie ex Fauc.

Potentilla anglica Laichard.

Potentilla apennina Ten. subsp. apennina

Potentilla argentea L. - Note: This species seems restricted to few localities of the Alps. Most records of $P$. argentea are likely to be referred to $P$. neglecta

Baumg. or P. argentea var. pseudocalabra Th. Wolf (see Paule et al. 2011).

Potentilla aurea L. subsp. aurea

Potentilla brauneana Hoppe

E Potentilla calabra Ten.

Potentilla caulescens $L$. subsp. caulescens

E Potentilla caulescens L. subsp. nebrodensis (Strobl ex Zimmeter) Arrigoni

Potentilla cinerea Chaix ex Vill.

Potentilla clusiana Jacq.

Potentilla collina Wibel

Potentilla crantzii (Crantz) Beck ex Fritsch subsp. crantzii

E Potentilla crassinervia Viv.

Potentilla detommasii Ten.

Potentilla erecta (L.) Raeusch.

Potentilla frigida Vill.

Potentilla grammopetala Moretti

Potentilla grandiflora $\mathrm{L}$.

Potentilla heptaphylla L. subsp. australis (Nyman) Gams

Potentilla heptaphylla L. subsp. heptaphylla

Potentilla incana G.Gaertn., B.Mey. \& Scherb.

Potentilla inclinata Vill.

E Potentilla johanniniana Goiran

Potentilla leucopolitana P.J.Müll.

Potentilla micrantha Ramond ex DC. 
Potentilla multifida L.

Potentilla neglecta Baumg. - Note: This species is likely more widespread than currently documented, as it is usually confused with P. argentea Ten.

Potentilla nitida $\mathrm{L}$.

Potentilla nivea $L$. subsp. nivea

Potentilla pedata Willd. ex Hornem.

Potentilla pensylvanica $\mathrm{L}$.

Potentilla pusilla Host

Potentilla recta $\mathrm{L}$. subsp. obscura (Willd.) Ces.

Potentilla recta $L$. subsp. recta

Potentilla reptans $\mathrm{L}$.

Potentilla rigoana Th. Wolf

Potentilla saxifraga Ardoino ex De Not.

Potentilla sterilis (L.) Garcke

Potentilla supina L. subsp. supina

Potentilla thuringiaca Bernh. ex Link

Potentilla tommasiniana F.W.Schultz

Potentilla valderia $\mathrm{L}$.

Potentilla verna $\mathrm{L}$.

Poterium rupiculum Boiss. \& Reut.

Poterium sanguisorba L. subsp. balearicum (Bourg. ex Nyman) Stace

Poterium sanguisorba $L$. subsp. sanguisorba

Poterium spinosum $\mathrm{L}$.

Poterium verrucosum Link ex G.Don

Prunus avium (L.) L.

Prunus brigantina Vill.

Prunus cocomilia Ten.

Prunus fruticosa Pall.

ET Prunus mahaleb L. subsp. cupaniana (Guss. ex É.Huet \& A.Huet) Arcang.

Prunus mahaleb L. subsp. mahaleb

Prunus padus L. subsp. borealis (A.Blytt) Nyman

Prunus padus L. subsp. padus

Prunus prostrata Labill.

Prunus spinosa L. subsp. spinosa

Prunus webbii (Spach) Vierh.

Pyracantha coccinea M.Roem.

ET Pyrus castribonensis Raimondo, Schicchi \& Mazzola

ET Pyrus ciancioi P.Marino, Spadaro, G.Castellano \& Raimondo

Pyrus communis L. subsp. pyraster (L.) Ehrh. - Note: This subspecies is hardly discernible from cultivated forms (i.e. P. communis s.str.; Roma-Marzio et al. 2016a).

Pyrus cordata Desv.

C Pyrus nivalis Jacq.

ET Pyrus sicanorum Raimondo, Schicchi \& P.Marino

Pyrus spinosa Forssk

ET Pyrus vallis-demonis Raimondo \& Schicchi

Rosa abietina Gren. ex Christ

Rosa agrestis Savi - Note: For the typification of this name, see Roma-Marzio et al. (2016b).

$\mathrm{T} \quad$ Rosa andegavensis Bastard

Rosa arvensis Huds.

Rosa balsamica Besser

Rosa caesia Sm.

Rosa canina L.

Rosa chavinii Rapin ex Reut.

Rosa corymbifera Borkh.

$\mathrm{T} \quad$ Rosa deseglisei Boreau

Rosa dumalis Bechst.

Rosa elliptica Tausch

Rosa gallica $\mathrm{L}$.

Rosa glauca Pourr.

Rosa heckeliana Tratt.

Rosa inodora Fr.

Rosa majalis Herrm.

Rosa marginata Wallr.

Rosa micrantha Borrer ex Sm.

Rosa mollis Sm.

Rosa montana Chaix

T Rosa nitidula Besser

Rosa pendulina $\mathrm{L}$.

Rosa pouzinii Tratt.

Rosa pseudoscabriuscula (R.Keller) Henker \& G.Schulze

Rosa pulverulenta M.Bieb.

Rosa rhaetica Gremli

Rosa rubiginosa L. - Note: Rosa gremlii Christ, recorded for TAA, is here regarded as a synonym of this species.

Rosa sempervirens L.

Rosa serafinii Viv.

Rosa sherardii Davies

Rosa sicula Tratt.

Rosa spinosissima $L$.

Rosa squarrosa (A.Rau) Boreau 
Rosa strobliana Burnat \& Gremli

Rosa stylosa Desv.

Rosa subcanina (Christ) Vuk.

Rosa subcollina (Christ) Vuk.

Rosa tomentosa $\mathrm{Sm}$.

Rosa uriensis Lagger \& Puget ex Cottet

Rosa villosa $\mathrm{L}$.

E Rosa viscosa Jan ex Guss.

Rubus acheruntinus Ten.

E Rubus aetneus Tornab.

E Rubus arrigonii Camarda

Rubus austrotirolensis (Sabr.) Dalla Torre \& Sarnth.

Rubus bicolor Opiz

Rubus bifrons Vest

Rubus caesius $\mathrm{L}$.

Rubus canescens DC.

Rubus constrictus Lefèvre \& P.J.Müll.

E

Rubus cupanianus Guss.

Rubus epipsilos Focke

Rubus euprepes Focke

Rubus fasciculatus P.J.Müll.

E Rubus festii H.E.Weber

Rubus glaucellus Sudre

Rubus grabowskii Weihe

Rubus guttiferus Trávn. \& Holub

Rubus henrici-egonis Holub

Rubus hirtus Waldst. \& Kit. group - Note: Including R. hirtus s.str. and R. glandulosus Bellardi.

Rubus idaeus L. subsp. idaeus

Rubus incanescens (DC.) Bertol.

ET Rubus laconensis Camarda

ET Rubus limbarae Camarda

Rubus macrophyllus Weihe \& Nees

Rubus montanus Lib. ex Lej.

Rubus nessensis Hall subsp. nessensis

Rubus obtusangulus Gremli

Rubus pericrispatus Holub \& Trávn.

Rubus perrobustus Holub

ET Rubus pignattii Camarda

Rubus plicatus Weihe \& Nees

Rubus praecox Bertol.

Rubus radula Weihe

Rubus saxatilis L.

Rubus sulcatus Vest

Rubus ulmifolius Schott

Rubus vestitus Weihe

Rubus sect. Corylifolii Lindl. - Note: We are not able to specify which species belonging to this section, besides R. festii H.E.Weber and R. fasciculatus P.J.Müll., occur in Italy.

E Sanguisorba dodecandra Moretti

Sanguisorba officinalis $L$.

Sibbaldia procumbens $L$.

Sorbus aria (L.) Crantz - Note: Recently, Sennikov and Kurtto (2017) proposed the splitting of Sorbus into several distinct genera. These authors relied on previous phylogenetic data (e.g. Li et al. 2012; Lo and Donoghue 2012), which highlighted the non-monophyletic nature of Sorbus in its traditional circumscription. However, in our opinion, the resolution and detail of the current phylogenetic knowledge within the tribe Pyreae is still not sufficient for a safe recircumscription of its genera. For this reason, we decided to provisionally maintain a wide concept of the genus Sorbus.

Sorbus aucuparia L. subsp. aucuparia

T Sorbus aucuparia L. subsp. glabrata (Wimm. \& Grab.) Hedl.

ET Sorbus aucuparia L. subsp. praemorsa (Guss.) Nyman

Sorbus austriaca (Beck) Hayek

ET Sorbus busambarensis G.Castellano, P.Marino, Raimondo \& Spadaro

Sorbus chamaemespilus (L.) Crantz

Sorbus domestica $\mathrm{L}$.

Sorbus graeca (Spach) Lodd. ex S.Schauer - Note: The taxonomically highly questionable Aria tergestina (H.Lindb.) Sennikov \& Kurtto is provisionally included here.

T Sorbus istriaca Kárpáti

ET Sorbus madoniensis Raimondo, G.Castellano, Bazan \& Schicchi

Sorbus mougeotii Soy.-Will. \& Godr.

Sorbus torminalis (L.) Crantz

Sorbus umbellata (Desf.) Fritsch

Spiraea cana Waldst. \& Kit.

Spiraea chamaedryfolia $\mathrm{L}$.

Spiraea decumbens W.D.J.Koch subsp. decumbens

Rhamnaceae

Taxonomic references: Oreoherzogia W.Vent (Hauenschild et al. 2016).

Frangula alnus Mill. subsp. alnus

Frangula rupestris (Scop.) Schur

Oreoherzogia alpina (L.) W.Vent 
Oreoherzogia fallax (Boiss.) W.Vent - Note: Records for TOS fall within the variability of O. alpina (Roma-Marzio et al. 2016a). Similar pictures may apply to other Italian regions.

E Oreoherzogia glaucophylla (Sommier) W.Vent

Oreoherzogia pumila (Turra) W.Vent subsp. pumila

Paliurus spina-christi Mill.

Rhamnus alaternus L. subsp. alaternus

Rhamnus cathartica $\mathrm{L}$.

Rhamnus intermedia Steud. \& Hochst.

Rhamnus lojaconoi Raimondo

Rhamnus lycioides L. subsp. oleoides (L.) Jahand. \& Maire

E Rhamnus persicifolia Moris

Rhamnus saxatilis Jacq.

Ziziphus lotus (L.) Lam. subsp. lotus

Elaeagnaceae

Hippophaë fluviatilis (Soest) Rivas Mart.

Ulmaceae

Ulmus glabra Huds.

C Ulmus laevis Pall.

Ulmus minor Mill. subsp. canescens (Melville) Browicz \& Ziel.

Ulmus minor Mill. subsp. minor

E Zelkova sicula Di Pasq., Garfi \& Quézel

Cannabaceae

Celtis australis L. subsp. australis

E Celtis tournefortii Lam. subsp. aetnensis (Tornab.) Raimondo \& Schicchi

E Celtis tournefortii Lam. subsp. asperrima (Lojac.) Raimondo \& Schicchi

Humulus Iupulus L.

Moraceae

Ficus carica L.

Urticaceae

Taxonomic references: Urtica dioica L. (Weigend 2006).

Parietaria cretica L.

Parietaria judaica L

Parietaria lusitanica L. subsp. Iusitanica

Parietaria mauritanica Durieu

Parietaria officinalis $\mathrm{L}$.

Soleirolia soleirolii (Req.) Dandy

Urtica atrovirens Req. ex Loisel.

Urtica dioica L. subsp. dioica

$\mathrm{T} \quad$ Urtica dioica L. subsp. pubescens (Ledeb.) Domin

Urtica membranacea Poir.

Urtica pilulifera $\mathrm{L}$.

E Urtica rupestris Guss.

Urtica urens $\mathrm{L}$.

Fagaceae

Castanea sativa Mill.

Fagus sylvatica L. subsp. sylvatica

Quercus cerris L.

Quercus coccifera $\mathrm{L}$.

T Quercus congesta C.Pres

T Quercus dalechampii Ten. - Note: For the taxonomy and distribution of this species, see Di Pietro et al. (2012, 2016).

Quercus frainetto Ten.

ET Quercus ichnusae Mossa, Bacch. \& Brullo

Quercus ilex L. subsp. ilex

C Quercus ithaburensis Decne. subsp. macrolepis (Kotschy) Hedge \& Yalt.

ET Quercus leptobalana Guss.

E Quercus petraea (Matt.) Liebl. subsp. austrotyrrhenica Brullo, Guarino \& Siracusa

Quercus petraea (Matt.) Liebl. subsp. petraea

Quercus pubescens Willd. subsp. pubescens

Quercus pyrenaica Willd.

Quercus robur L. subsp. brutia (Ten.) O.Schwarz

Quercus robur L. subsp. robur

Quercus suber L.

Quercus trojana Webb subsp. trojana

Juglandaceae

C Juglans regia L. - Note: According to Accorsi et al. (1989) and Mercuri et al. (2013), this species could be native at least in VEN.

Betulaceae

Alnus alnobetula (Ehrh.) K.Koch

E Alnus cordata (Loisel.) Duby

Alnus glutinosa (L.) Gaertn.

Alnus incana (L.) Moench 
ET Betula etnensis Raf.

Betula nana L. subsp. nana

Betula pendula Roth

Betula pubescens Ehrh.

Carpinus betulus $\mathrm{L}$.

Carpinus orientalis Mill. subsp. orientalis

Corylus avellana $\mathrm{L}$.

Ostrya carpinifolia Scop.

Coriariaceae

Coriaria myrtifolia L.

Cucurbitaceae

Bryonia acuta Desf.

C Bryonia alba L.

Bryonia dioica Jacq.

Bryonia marmorata E.Petit

Citrullus colocynthis (L.) Schrad.

Ecballium elaterium (L.) A.Rich.

Celastraceae

Euonymus europaeus $\mathrm{L}$.

Euonymus latifolius (L.) Mill.

Euonymus verrucosus Scop.

Parnassia palustris L. subsp. palustris

Oxalidaceae

Oxalis acetosella L.

Oxalis corniculata $\mathrm{L}$.

Violaceae

E Viola aethnensis (Ging. \& DC.) Strobl subsp. aethnensis

E Viola aethnensis (Ging. \& DC.) Strobl subsp. messanensis (W.Becker) Merxm. \& Lippert

E Viola aethnensis (Ging. \& DC.) Strobl subsp. splendida (W.Becker) Merxm. \& Lippert

Viola alba Besser subsp. alba

T Viola alba Besser subsp. dehnhardtii (Ten.) W.Becker

Viola ambigua Waldst. \& Kit.

Viola arborescens $\mathrm{L}$.

Viola argenteria Moraldo \& Forneris

Viola arvensis Murray subsp. arvensis

Viola arvensis Murray subsp. megalantha Nauenb.

$E$

Viola bertolonii Pio

Viola biflora $\mathrm{L}$.

Viola calcarata L. subsp. calcarata

Viola calcarata L. subsp. villarsiana (Roem. \& Schult.) Merxm.

Viola canina L. subsp. canina

E Viola cassinensis Strobl subsp. cassinensis

ET Viola cassinensis Strobl subsp. pseudogracilis (A.Terracc.) Bartolucci, Galasso \& Wagens. - Note: For the nomenclature of this species, see Bartolucci et al. (2018).

Viola cavillieri W.Becker

Viola cenisia $\mathrm{L}$.

Viola collina Besser

E Viola comollia Massara

E Viola corsica Nyman subsp. ilvensis (W.Becker) Merxm.

E Viola culminis F.Fen. \& Moraldo

E Viola dubyana Burnat ex Gremli

Viola elatior $\mathrm{Fr}$.

E Viola etrusca Erben

E Viola eugeniae Parl. subsp. eugeniae

ET Viola eugeniae Parl. subsp. levieri (Parl.) Arcang.

ET Viola ferrarinii Moraldo \& Ricceri

Viola hirta L.

Viola hymettia Boiss. \& Heldr.

Viola jordanii Hanry

Viola kitaibeliana Schult.

Viola laricicola Marcussen

E Viola limbarae (Merxm. \& W.Lippert) Arrigoni

E Viola magellensis Porta \& Rigo ex Strobl

E Viola merxmuelleri Erben

Viola mirabilis $\mathrm{L}$.

E Viola nebrodensis C.Presl

Viola odorata $\mathrm{L}$.

Viola palustris $\mathrm{L}$.

Viola parvula Tineo

Viola pinnata $\mathrm{L}$.

Viola pumila Chaix

Viola pyrenaica Ramond ex DC.

Viola reichenbachiana Jord. ex Boreau 
Viola riviniana Rchb. subsp. riviniana

Viola rupestris F.W.Schmidt subsp. rupestris

Viola suavis M.Bieb. subsp. adriatica (Freyn) Hasler

Viola suavis M.Bieb. subsp. suavis

Viola thomasiana Songeon \& E.P.Perrier

E Viola tineoorum Erben \& Raimondo

$\mathrm{T}$ Viola tricolor L. subsp. saxatilis (F.W.Schmidt) Jan

Viola tricolor L. subsp. tricolor

E Viola ucriana Erben \& Raimondo

Viola valderia All.

Salicaceae

Populus alba L.

Populus canescens (Aiton) Sm.

T Populus nigra L. subsp. neapolitana (Ten.) Asch. \& Graebn.

Populus nigra L. subsp. nigra

Populus tremula L.

Salix alba L.

Salix alpina Scop.

Salix amplexicaulis Bory

Salix apennina A.K.Skvortsov

Salix appendiculata Vill.

ET Salix arrigonii Brullo

Salix atrocinerea Brot. subsp. atrocinerea

Salix aurita L.

Salix breviserrata Flod.

E Salix brutia Brullo \& Spamp.

Salix caesia Vill.

Salix caprea $\mathrm{L}$.

Salix cinerea L.

Salix crataegifolia Bertol.

Salix daphnoides Vill.

Salix eleagnos Scop.

Salix foetida Schleich. ex DC.

Salix glabra Scop.

Salix glaucosericea Flod.

E Salix gussonei Brullo \& Spamp.

Salix hastata L.

Salix hegetschweileri Heer

Salix helvetica Vill.

Salix herbacea $\mathrm{L}$.

E Salix ionica Brullo, Scelsi \& Spamp.

Salix Iaggeri Wimm.

Salix mielichhoferi Saut.

Salix myrsinifolia Salisb.

E Salix nebrodensis C.Brullo, Brullo, Cambria \& Giusso

E Salix oropotamica Brullo, Scelsi \& Spamp.

Salix pedicellata Desf.

Salix pentandra $\mathrm{L}$.

Salix purpurea L. subsp. angustior Lautenschl.

ET Salix purpurea L. subsp. eburnea (Borzí) Cif. \& Giacom. ex Pignatti

Salix purpurea L. subsp. purpurea

Salix repens $L$. subsp. repens

Salix reticulata $\mathrm{L}$.

Salix retusa $\mathrm{L}$.

Salix rosmarinifolia $\mathrm{L}$.

Salix serpillifolia Scop.

Salix triandra L. subsp. triandra

E Salix tyrrhenica Brullo, Scelsi \& Spamp.

Salix waldsteiniana Willd.

Linaceae

Linum alpinum Jacq.

Linum campanulatum L.

Linum capitatum Kit. ex Schult. subsp. serrulatum (Bertol.) Hartvig

Linum catharticum L. subsp. catharticum

T Linum catharticum L. subsp. suecicum Hayek

Linum corymbulosum Rchb.

Linum decumbens Desf.

Linum elegans Spruner ex Boiss. - Note: For the distribution and morphology of this species, see Wagensommer et al. (2017).

Linum flavum $\mathrm{L}$.

E Linum katiae Peruzzi - Note: For the taxonomy and distribution of this species, see Peruzzi (2011).

$\mathrm{T} \quad$ Linum maritimum L. subsp. ligusticum (Rouy) P.Fourn.

Linum maritimum L. subsp. maritimum

E Linum mulleri Moris

Linum narbonense $\mathrm{L}$

Linum nodiflorum L.

E Linum punctatum C.Presl subsp. punctatum 


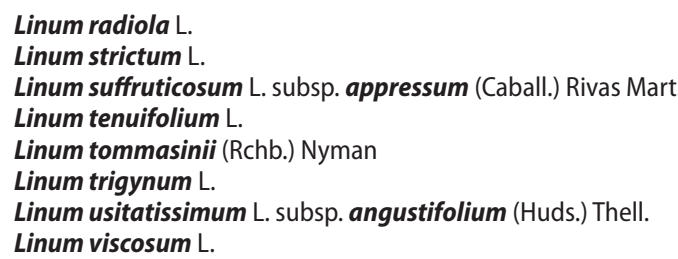

Hypericaceae

Hypericum aegypticum $\mathrm{L}$.

Hypericum androsaemum $\mathrm{L}$.

Hypericum annulatum Moris

Hypericum australe Ten.

ET Hypericum barbatum Jacq. subsp. calabricum (Spreng.) Peruzzi \& N.G.Passal.

Hypericum coris $L$.

E Hypericum corsicum Steud.

Hypericum elodes L. - Note: For the biology and distribution of this species, see Carta et al. (2016) and literature cited therein.

E Hypericum hircinum L. subsp. hircinum

Hypericum hircinum L. subsp. majus (Aiton) N.Robson

Hypericum hirsutum $\mathrm{L}$.

Hypericum humifusum L.

Hypericum hyssopifolium Chaix

Hypericum maculatum Crantz subsp. maculatum

Hypericum montanum $\mathrm{L}$.

Hypericum nummularium $\mathrm{L}$.

Hypericum perfoliatum $\mathrm{L}$.

Hypericum perforatum L. subsp. perforatum

Hypericum perforatum $L$. subsp. veronense (Schrank) Ces.

Hypericum pubescens Boiss.

Hypericum richeri Vill. subsp. richeri

E Hypericum scruglii Bacch., Brullo \& Salmeri

Hypericum spruneri Boiss.

Hypericum tetrapterum $\mathrm{Fr}$

Hypericum tomentosum $\mathrm{L}$.

Hypericum triquetrifolium Turra

Elatinaceae

Elatine alsinastrum $\mathrm{L}$.

Elatine campylosperma Seub.

Elatine gussonei (Sommier) Brullo, Lanfr., Pavone \& Ronsisv.

Elatine hexandra (Lapierre) DC. - Note: It is an allopolyploid species, probably originated in Spain or Morocco (Razifard et al. 2017).

Elatine hydropiper L.

Elatine macropoda Guss.

Elatine triandra Schkuhr

Euphorbiaceae

Taxonomic references: Euphorbia barrelieri Savi group (Frajman and Schönswetter 2017).

Chrozophora tinctoria (L.) A.Juss.

Euphorbia akenocarpa Guss.

Euphorbia aleppica L.

Euphorbia amygdaloides $\mathrm{L}$.

Euphorbia angulata Jacq.

Euphorbia apios L.

Euphorbia barrelieri Savi - Note: According to Frajman and Schönswetter (2017), E. thessala (Formanek) Degen \& Dorfl. [三E. barrelieri Savi subsp. thessala (Formánek) Bornm.] does not occur in Italy.

Euphorbia biumbellata Poir.

Euphorbia bivonae Steud. subsp. bivonae

Euphorbia carniolica Jacq.

Euphorbia ceratocarpa Ten.

Euphorbia chamaesyce $\mathrm{L}$.

Euphorbia characias L.

E Euphorbia corallioides L.

Euphorbia cuneifolia Guss.

Euphorbia cyparissias L.

Euphorbia dendroides $\mathrm{L}$.

Euphorbia dulcis L.

Euphorbia esula L. subsp. esula

Euphorbia esula L. subsp. tommasiniana (Bertol.) Kuzmanov

Euphorbia exigua L. subsp. exigua

Euphorbia falcata L. subsp. falcata

Euphorbia flavicoma DC. subsp. flavicoma

Euphorbia fragifera Jan

E Euphorbia gasparrinii Boiss. subsp. gasparrinii

E Euphorbia gasparrinii Boiss. subsp. samnitica (Fiori) Pignatti

Euphorbia gayi Salis

Euphorbia helioscopia L. subsp. helioscopia

Euphorbia hirsuta L. 
Euphorbia hyberna L. subsp. canuti (Parl.) Tutin

Euphorbia hyberna L. subsp. gibelliana (Peola) Raffaelli

Euphorbia hyberna L. subsp. insularis (Boiss.) Briq.

Euphorbia illirica Lam.

Euphorbia kerneri Huter ex A.Kern.

Euphorbia lagascae Spreng.

Euphorbia lucida Waldst. \& Kit.

E Euphorbia meuselii Geltman

Euphorbia myrsinites L. subsp. myrsinites

ET Euphorbia nicaeensis All. subsp. japygica (Ten.) Arcang.

Euphorbia nicaeensis All. subsp. nicaeensis

Euphorbia palustris L.

E Euphorbia papillaris (Boiss.) Raffaelli \& Ricceri

Euphorbia paralias L.

Euphorbia peplis L.

Euphorbia peplus L.

Euphorbia phymatosperma Boiss. subsp. cernua (Coss. \& Durieu ex Boiss.) Vindt

Euphorbia pithyusa L. subsp. cupanii (Guss. ex Bertol.) Radcl.-Sm.

Euphorbia pithyusa L. subsp. pithyusa

Euphorbia platyphyllos L.

Euphorbia pterococca Brot.

Euphorbia rigida M.Bieb.

Euphorbia segetalis $\mathrm{L}$.

Euphorbia seguieriana Neck. subsp. seguieriana

Euphorbia semiperfoliata Viv.

Euphorbia serrata L.

Euphorbia spinosa $\mathrm{L}$.

Euphorbia stricta L.

Euphorbia sulcata Lens ex Loisel.

Euphorbia taurinensis All.

Euphorbia terracina $\mathrm{L}$.

Euphorbia valliniana Belli

E Euphorbia variabilis Ces.

Euphorbia verrucosa $\mathrm{L}$.

Mercurialis annua $\mathrm{L}$.

E Mercurialis corsica Coss. \& Kralik

Mercurialis ovata Sternb. \& Hoppe

Mercurialis perennis $\mathrm{L}$.

Phyllanthaceae

Andrachne telephioides $\mathrm{L}$.

Geraniaceae

Erodium acaule (L.) Bech. \& Thell.

Erodium alnifolium Guss.

E Erodium alpinum (Burm.f.) L'Hér

Erodium botrys (Cav.) Bertol.

Erodium chium (L.) Willd.

Erodium ciconium (L.) L'Hér.

Erodium cicutarium (L.) L'Hér.

E Erodium corsicum Léman

Erodium gruinum (L.) L'Hér

Erodium laciniatum (Cav.) Willd. subsp. Iaciniatum - Note: According to Domina et al. (2017b), we include E. soluntinum Tod. here, as a heterotypic synonym.

Erodium lebelii Jord. subsp. marcuccii (Parl.) Guitt.

Erodium malacoides (L.) L'Hér. subsp. malacoides

Erodium maritimum (L.) L'Hér.

Erodium moschatum (L.) L'Hér.

Erodium nervulosum L'Hér.

Erodium neuradifolium Delile ex Godr.

Erodium salzmannii Delile

Geranium argenteum $\mathrm{L}$.

Geranium asphodeloides Burm.f. subsp. asphodeloides

E Geranium austroapenninum Aedo

Geranium bohemicum L.

Geranium columbinum $\mathrm{L}$.

Geranium dissectum

Geranium divaricatum Ehrh.

Geranium Ianuginosum Lam.

Geranium lucidum L.

Geranium macrorrhizum L.

Geranium molle L.

Geranium nodosum $\mathrm{L}$.

Geranium palustre

Geranium phaeum L.

Geranium pratense L. subsp. pratense

Geranium purpureum Vill.

Geranium pusillum

Geranium pyrenaicum Burm.f. subsp. pyrenaicum 
Geranium reflexum $\mathrm{L}$.

Geranium rivulare Vill.

Geranium robertianum $\mathrm{L}$.

Geranium rotundifolium $\mathrm{L}$.

Geranium sanguineum $L$.

Geranium sibiricum L.

Geranium sylvaticum $\mathrm{L}$.

Geranium tuberosum L. subsp. tuberosum

Geranium versicolor $\mathrm{L}$.

Onagraceae

Taxonomic references: Chamaenerion Ség. (Sennikov 2011).

Chamaenerion angustifolium (L.) Scop.

Chamaenerion dodonaei (Vill.) Schur ex Fuss

Chamaenerion fleischeri (Hochst.) Fritsch

Circaea alpina L. subsp. alpina

Circaea lutetiana $\mathrm{L}$. subsp. Iutetiana

Epilobium alpestre (Jacq.) Krock.

Epilobium alsinifolium Vill.

Epilobium anagallidifolium Lam.

Epilobium collinum C.C.Gmel.

Epilobium hirsutum $\mathrm{L}$.

Epilobium Ianceolatum Sebast. \& Mauri

Epilobium montanum L.

Epilobium nutans F.W.Schmidt

Epilobium obscurum Schreb.

Epilobium palustre $\mathrm{L}$.

Epilobium parviflorum Schreb.

Epilobium roseum Schreb. subsp. roseum

$\mathrm{T} \quad$ Epilobium tetragonum L. subsp. Iamyi (F.W.Schultz) Nyman

Epilobium tetragonum L. subsp. tetragonum

T Epilobium tetragonum L. subsp. tournefortii (Michalet) H.Lév.

Ludwigia palustris (L.) Elliott

Lythraceae

Lythrum acutangulum Lag.

Lythrum hyssopifolia L.

Lythrum junceum Banks \& Sol.

Lythrum salicaria $L$.

Lythrum thesioides M.Bieb.

Lythrum thymifolia L.

Lythrum tribracteatum Salzm. ex Spreng.

Lythrum virgatum $\mathrm{L}$.

Middendorfia borysthenica (Schrank) Trautv.

Peplis portula $\mathrm{L}$.

Trapa natans $\mathrm{L}$.

Myrtaceae

Myrtus communis L.

Staphyleaceae

Staphylea pinnata $\mathrm{L}$.

Nitrariaceae

Peganum harmala $\mathrm{L}$.

Anacardiaceae

Cotinus coggygria Scop.

Pistacia lentiscus $L$.

Pistacia terebinthus L. subsp. terebinthus

C Rhus coriaria L.

Rhus pentaphylla (Jacq.) Desf.

Rhus tripartita (Ucria) Grande

Sapindaceae

Acer campestre L.

E $\quad$ Acer cappadocicum Gled. subsp. Iobelii (Ten.) A.E.Murray

Acer monspessulanum $L$. subsp. monspessulanum

T Acer opalus Mill. subsp. obtusatum (Waldst. \& Kit. ex Willd.) Gams - Note: Herbarium specimens from TOS show often morphological features intermediate among A. opalus s.str. and A. opalus subp. obtusatum (Roma-Marzio et al. 2016a).

Acer opalus Mill. subsp. opalus

Acer platanoides $\mathrm{L}$.

Acer pseudoplatanus $\mathrm{L}$.

Rutaceae

Cneorum tricoccon $\mathrm{L}$

Dictamnus albus $\mathrm{L}$. 
Haplophyllum patavinum (L.) G.Don

Ruta angustifolia Pers.

Ruta chalepensis L.

Ruta graveolens $\mathrm{L}$.

Ruta montana (L.) L.

Thymelaeaceae

Daphne alpina L. subsp. alpina

Daphne alpina L. subsp. scopoliana Urbani

Daphne blagayana Freyer

Daphne cneorum L.

Daphne gnidium L.

Daphne laureola L.

Daphne mezereum L.

Daphne oleoides Schreb. subsp. oleoides

E Daphne petraea Leyb.

E Daphne reichsteinii Landolt \& E.J.P.Hauser

Daphne sericea Vahl

Daphne striata Tratt.

Thymelaea dioica (Gouan) All.

Thymelaea gussonei Boreau

Thymelaea hirsuta (L.) Endl.

Thymelaea passerina (L.) Coss. \& Germ.

Thymelaea tartonraira (L.) All. subsp. tartonraira

Cistaceae

Cistus albidus L.

Cistus clusii Dunal

Cistus creticus L. subsp. corsicus (Loisel.) Greuter \& Burdet

Cistus creticus L. subsp. creticus

Cistus creticus L. subsp. eriocephalus (Viv.) Greuter \& Burdet

Cistus crispus $\mathrm{L}$.

Cistus laurifolius L. subsp. Iaurifolius - Note: For the biology and distribution of this species, see Astuti et al. (2017b) and literature cited therein.

Cistus monspeliensis L.

Cistus parviflorus Lam

Cistus salviifolius L.

Fumana arabica (L.) Spach

Fumana ericifolia Wallr.

Fumana juniperina (Lag. ex Dunal) Pau

Fumana laevipes (L.) Spach

Fumana laevis (Cav.) Pau

Fumana procumbens (Dunal) Gren. \& Godr.

Fumana scoparia Pomel

Fumana thymifolia (L.) Spach ex Webb

Halimium halimifolium (L.) Willk. subsp. halimifolium

Helianthemum aegyptiacum (L.) Mill.

Helianthemum apenninum (L.) Mill. subsp. apenninum

Helianthemum caput-felis Boiss.

Helianthemum cinereum (Cav.) Pers. subsp. rotundifolium (Dunal) Greuter \& Burdet

Helianthemum croceum (Desf.) Pers.

E Helianthemum farinulentum Lacaita

Helianthemum jonium Lacaita \& Grosser

Helianthemum ledifolium (L.) Mill.

Helianthemum leptophyllum Dunal

Helianthemum lippii (L.) Dum.Cours.

Helianthemum Iunulatum (All.) DC.

Helianthemum morisianum Bertol.

Helianthemum nummularium (L.) Mill. subsp. berteroanum (Bertol.) Breistr.

Helianthemum nummularium (L.) Mill. subsp. glabrum (W.D.J.Koch) Wilczek

Helianthemum nummularium (L.) Mill. subsp. grandiflorum (Scop.) Schinz \& Thell.

Helianthemum nummularium (L.) Mill. subsp. nummularium

Helianthemum nummularium (L.) Mill. subsp. obscurum (Čelak.) Holub

Helianthemum nummularium (L.) Mill. subsp. semiglabrum (Badarò) M.Proctor

Helianthemum nummularium (L.) Mill. subsp. tomentosum (Scop.) Schinz \& Thell.

Helianthemum oelandicum (L.) Dum.Cours. subsp. allionii (Tineo) Greuter \& Burdet

Helianthemum oelandicum (L.) Dum.Cours. subsp. alpestre (Jacq.) Ces.

Helianthemum oelandicum (L.) Dum.Cours. subsp. incanum (Willk.) G.López

Helianthemum oelandicum (L.) Dum.Cours. subsp. italicum (L.) Ces.

E Helianthemum oelandicum (L.) Dum.Cours. subsp. nebrodense (Heldr. ex Guss.) Greuter \& Burdet

DD Helianthemum oelandicum (L.) Dum.Cours. subsp. pourretii (Timb.-Lagr.) Greuter \& Burdet

Helianthemum pilosum (L.) Pers.

Helianthemum salicifolium (L.) Mill.

Helianthemum sanguineum (Lag.) Lag. ex Dunal

E Helianthemum sicanorum Brullo, Giusso \& Sciandr.

Helianthemum syriacum (Jacq.) Dum.Cours. subsp. thibaudii (Pers.) Meikle - Note: Possibly extinct in Italy.

Tuberaria acuminata (Viv.) Grosser

Tuberaria guttata (L.) Fourr. 
Tuberaria inconspicua (Pers.) Willk.

Tuberaria lignosa (Sweet) Samp.

Tuberaria praecox Grosser

Tuberaria villosissima (Pomel) Grosser

Cytinaceae

Cytinus hypocistis (L.) L.

Cytinus ruber Fourr. ex Fritsch

Malvaceae

Taxonomic references: Malva L. (incl. Lavatera L.) (Banfi et al. 2005; Molero and Montserrat 2005; lamonico 2010; Valdés 2011; Conti and Bartolucci 2012; lamonico et al. 2015).

Althaea cannabina $\mathrm{L}$.

Althaea officinalis $\mathrm{L}$.

Hibiscus pentacarpos $\mathrm{L}$.

Malope malacoides $L$. subsp. malacoides

Malope malacoides L. subsp. tripartita (Boiss. \& Reut.) Maire

Malva agrigentina (Tineo) Soldano, Banfi \& Galasso

Malva alcea $\mathrm{L}$.

Malva arborea (L.) Webb \& Berthel.

Malva cretica Cav. subsp. althaeoides (Cav.) Bég.

Malva cretica Cav. subsp. cretica

Malva lusitanica (L.) Valdés subsp. Iusitanica

E Malva lusitanica (L.) Valdés subsp. pallescens (Moris) Valdés

Malva moschata $\mathrm{L}$.

Malva multiflora (Cav.) Soldano, Banfi \& Galasso

Malva neglecta Wallr.

Malva nicaeensis All.

Malva olbia (L.) Alef.

Malva oxyloba Boiss.

Malva parviflora $\mathrm{L}$.

Malva punctata (All.) Alef.

Malva pusilla Sm.

Malva setigera K.F.Schimp. \& Spenn.

E Malva stenopetala (Coss. \& Durieu ex Batt.) Soldano, Banfi \& Galasso subsp. plazzae (Atzei) lamonico, Bartolucci \& Peruzzi - Note: For the taxonomy of this taxon, see lamonico (2014a).

Malva subovata (DC.) Molero \& J.M.Monts. subsp. bicolor (Rouy) lamonico

Malva subovata (DC.) Molero \& J.M.Monts. subsp. subovata - Note: For the taxonomy and distribution of this species, see lamonico (2016a).

Malva sylvestris $\mathrm{L}$.

Malva thuringiaca (L.) Vis.

Malva trimestris (L.) Salisb.

Malva unguiculata (Desf.) Alef.

Tilia cordata Mill.

T Tilia platyphyllos Scop. subsp. cordifolia (Besser) C.K.Schneid.

Tilia platyphyllos Scop. subsp. platyphyllos

T Tilia platyphyllos Scop. subsp. pseudorubra C.K.Schneid.

Resedaceae

Reseda alba L. subsp. alba

T Reseda alba L. subsp. hookeri (Guss.) Arcang.

Reseda lutea L. subsp. Iutea

Reseda luteola L.

Reseda phyteuma L. subsp. phyteuma

Sesamoides interrupta (Boreau) G.López

DD Sesamoides purpurascens (L.) G.López

Sesamoides spathulifolia (Revelière ex Boreau) Rothm.

Capparaceae

Capparis orientalis Veill.

Capparis sicula Veill

Capparis spinosa L. - Note: Probably C. spinosa is a species of hybrid origin, involving C. orientalis Veill. and C. sicula Veill. as parental species (Inocencio et al. 2006).

Brassicaceae

Taxonomic references: Aubrieta Adans. (Koch et al. 2017); Erysimum L. (Peccenini 2012; Peccenini and Polatschek 2014, 2016); Malcolmia W.T.Aiton s.str., Maresia Pomel, Marcus-Kochia Al-Shehbaz, and Strigosella Boiss. (Al-Shehbaz et al. 2014).

Aethionema marginatum (Lapeyr.) Montemurro

Aethionema saxatile (L.) R.Br.

Aethionema thomasianum J.Gay

Alliaria petiolata (M.Bieb.) Cavara \& Grande

Alyssoides utriculata (L.) Medik. subsp. utriculata

Alyssum alyssoides (L.) L.

E Alyssum cuneifolium Ten.

Alyssum desertorum Stapf - Note: For the Italian distribution of this species, see Bertolli and Prosser (2014), Bartolucci and Conti (2016).

Alyssum diffusum Ten. subsp. calabricum Španiel, Marhold, N.G.Passal. \& Lihová

Alyssum diffusum Ten. subsp. diffusum

Alyssum diffusum Ten. subsp. garganicum Španiel, Marhold, N.G.Passal. \& Lihová 
Alyssum doerfleri Degen - Note: For the distribution and taxonomy of this species, see Bernardo et al. (2018).

Alyssum minutum Schltdl. ex DC.

Alyssum montanum L. - Note: This species is excluded from the Italian flora by Španiel et al. (2011, 2012) and Zozomová-Lihová et al. (2014). Some doubtful populations, previously indicated as A. montanum, are provisionally still included here.

Alyssum orophilum Jord. \& Fourr.

Alyssum repens Baumg.

Alyssum siculum Jord.

Alyssum simplex Rudolphi

Alyssum strigosum Banks \& Sol. subsp. strigosum

Alyssum wulfenianum Bernh. subsp. ovirense (A.Kern.) Magauer, Schönsw. \& Frajman

Alyssum wulfenianum Bernh. subsp. wulfenianum

Arabidopsis arenosa (L.) Lawalrée

Arabidopsis halleri (L.) O'Kane \& Al-Shehbaz subsp. halleri

Arabidopsis halleri (L.) O'Kane \& Al-Shehbaz subsp. ovirensis (Wulfen) O'Kane \& Al-Shehbaz

Arabidopsis pedemontana (Boiss.) O'Kane \& Al-Shehbaz

Arabidopsis petraea (L.) V.I.Dorof.

Arabidopsis thaliana (L.) Heynh.

Arabis allionii DC.

Arabis alpina L. subsp. alpina

Arabis alpina L. subsp. caucasica (Willd.) Briq.

Arabis arabiformis (Hohenw.) Soldano

Arabis auriculata Lam.

Arabis bellidifolia Crantz subsp. bellidifolia

Arabis bellidifolia Crantz subsp. stellulata (Bertol.) Greuter \& Burdet

Arabis caerulea All.

Arabis ciliata Clairv.

Arabis collina Ten. subsp. collina

Arabis collina Ten subsp rosea (DC.) Minuto

Arabis hirsuta (L.) Scop.

ET Arabis madonia C.Presl

Arabis nova Vill. subsp. nova

Arabis planisiliqua (Pers.) Rchb.

Arabis sagittata (Bertol.) DC.

Arabis serpillifolia Vill.

Arabis soyeri Reut. \& A.Huet subsp. subcoriacea (Gren.) Breistr.

Arabis surculosa N.Terracc.

Arabis verna (L.) R.Br.

E Aubrieta columnae Guss. subsp. columnae

E Aubrieta columnae Guss. subsp. italica (Boiss.) Mattf.

E Aubrieta columnae Guss. subsp. sicula (Steven) Phitos

Aurinia leucadea (Guss.) K.Koch

Aurinia petraea (Ard.) Schur

Aurinia saxatilis (L.) Desv. subsp. megalocarpa (Hausskn.) T.R.Dudley

Aurinia sinuata (L.) Griseb.

Barbarea bracteosa Guss.

Barbarea intermedia Boreau

Barbarea rupicola Moris

Barbarea sicula C.Pres

Barbarea stricta Andrz.

Barbarea verna (Mill.) Asch.

Barbarea vulgaris $\mathrm{R} . \mathrm{Br}$.

Berteroa incana (L.) DC.

Berteroa mutabilis (Vent.) DC.

Berteroa obliqua (Sm.) DC

Biscutella ambigua DC.

Biscutella apuana Raffaelli

Biscutella brevicaulis Jord.

Biscutella cichoriifolia Loisel.

Biscutella coronopifolia $\mathrm{L}$.

Biscutella didyma L. subsp. apula Nyman

Biscutella didyma L. subsp. didyma

Biscutella incana Ten.

E Biscutella laevigata L. subsp. australis Raffaelli \& Baldoin Biscutella laevigata L. subsp. laevigata

Biscutella laevigata L. subsp. Iucida (Balb. ex DC.) Arcang.

Biscutella laevigata L. subsp. ossolana Raffaelli \& Baldoin

Biscutella laevigata L. subsp. prinzerae Raffaelli \& Baldoin

Biscutella laevigata L. subsp. raffaelliana Galasso \& Banfi

Biscutella maritima Ten.

Biscutella mollis Loisel.

Biscutella morisiana Raffaelli

Biscutella pichiana Raffaelli subsp. ilvensis Raffaelli

Biscutella pichiana Raffaelli subsp. pichiana

Biscutella prealpina Raffaelli \& Baldoin

Bivonaea lutea (Biv.) DC.

E Brassica baldensis (Prosser \& Bertolli) Prosser \& Bertolli

Brassica fruticulosa Cirillo subsp. fruticulosa

Brassica glabrescens Poldini 
Brassica gravinae Ten.

Brassica incana Ten.

Brassica insularis Moris

Brassica macrocarpa Guss.

Brassica montana Pourr.

C Brassica nigra (L.) W.D.J.Koch

Brassica procumbens (Poir.) O.E.Schulz

Brassica rapa L. subsp. campestris (L.) A.R.Clapham

Brassica repanda (Willd.) DC.

E Brassica rupestris Raf. subsp. hispida Raimondo \& Mazzola

E Brassica rupestris Raf. subsp. rupestris

Brassica souliei (Batt.) Batt. subsp. amplexicaulis (Desf.) Greuter \& Burdet

Brassica tournefortii Gouan

E Brassica trichocarpa C.Brullo, Brullo, Giusso, llardi

E Brassica tyrrhena Giotta, Piccitto \& Arrigoni

E Brassica villosa Biv. subsp. bivonana (Mazzola \& Raimondo) Raimondo \& Mazzola

E Brassica villosa Biv. subsp. brevisiliqua (Raimondo \& Mazzola) Raimondo \& Geraci

E Brassica villosa Biv. subsp. drepanensis (Caruel) Raimondo \& Mazzola

E Brassica villosa Biv. subsp. tineoi (Lojac.) Raimondo \& Mazzola

E Brassica villosa Biv. subsp. villosa

Braya alpina Sternb. \& Hoppe

Bunias erucago L.

Cakile maritima Scop. subsp. maritima

Calepina irregularis (Asso) Thell.

Camelina alyssum (Mill.) Thell. subsp. alyssum - Note: Possibly extinct in Italy.

DD Camelina alyssum (Mill.) Thell. subsp. integerrima (Čelak.) Smejkal

Camelina microcarpa Andrz. ex DC.

Camelina sativa (L.) Crantz

Capsella bursa-pastoris (L.) Medik. subsp. bursa-pastoris

T Capsella rubella Reut.

Cardamine alpina Willd.

Cardamine amara $L$. subsp. amara

Cardamine amara $\mathrm{L}$. subsp. austriaca Marhold

Cardamine amporitana Sennen \& Pau

E Cardamine apennina Lihová \& Marhold

Cardamine asarifolia L.

E Cardamine battagliae Cesca \& Peruzzi

Cardamine bulbifera (L.) Crantz

Cardamine chelidonia $\mathrm{L}$.

E Cardamine dubia Nicotra

Cardamine enneaphyllos (L.) Crantz

Cardamine flexuosa With.

Cardamine glauca Spreng. ex DC. subsp. glauca

Cardamine graeca $\mathrm{L}$.

E Cardamine granulosa All.

Cardamine heptaphylla (Vill.) O.E.Schulz

Cardamine hirsuta L.

Cardamine impatiens $L$. subsp. impatiens

Cardamine kitaibelii Bech.

Cardamine matthioli Moretti

E Cardamine monteluccii Brilli-Catt. \& Gubellini

Cardamine parviflora $\mathrm{L}$.

Cardamine pentaphyllos (L.) Crantz

Cardamine plumieri Vill.

Cardamine pratensis $\mathrm{L}$.

Cardamine resedifolia $\mathrm{L}$.

E Cardamine silana Marhold \& Perný

Cardamine trifolia L.

Carrichtera annua (L.) DC.

Clypeola jonthlaspi $\mathrm{L}$.

Coincya monensis (L.) Greuter \& Burdet subsp. cheiranthos (Franco) Aedo, Leadlay \& Muñoz Garm.

Coincya richeri (Vill.) Greuter \& Burdet

Conringia austriaca (Jacq.) Sweet

Crambe hispanica L.

Crambe tataria Sebeók

Descurainia sophia (L.) Webb ex Prantl

Diplotaxis erucoides (L.) DC. subsp. erucoides

Diplotaxis harra (Forssk.) Boiss. subsp. crassifolia (Raf.) Maire

Diplotaxis muralis (L.) DC.

Diplotaxis scaposa DC

Diplotaxis tenuifolia (L.) DC.

Diplotaxis viminea (L.) DC.

Draba aizoides $\mathrm{L}$. subsp. aizoides

Draba aspera Bertol.

Draba dolomitica Buttler

Draba dubia Suter subsp. dubia

Draba fladnizensis Wulfen

Draba hoppeana Rchb. 
Draba ladina Braun-Blanq.

Draba nemorosa L.

Draba siliquosa M.Bieb.

Draba stylaris J.Gay ex W.D.J.Koch

Draba tomentosa Clairv. subsp. tomentosa

\section{Iberis integerrima Moris}

Draba verna L. subsp. praecox (Steven) Rouy \& Foucaud

Draba verna L. subsp. spathulata (Láng) Rouy \& Foucaud

Draba verna $L$. subsp. verna

Drabella muralis (L.) Fourr.

Eruca vesicaria (L.) Cav.

Erucastrum gallicum (Willd.) O.E.Schulz

Erucastrum nasturtiifolium (Poir.) O.E.Schulz subsp. benacense F.Martini \& F.Fen.

Erucastrum nasturtiifolium (Poir.) O.E.Schulz subsp. nasturtiifolium

Erucastrum palustre (Pirona) Vis

Erucastrum virgatum C.Presl subsp. virgatum

Erysimum apenninum Peccenini \& Polatschek

Erysimum aurantiacum (Leyb.) Leyb.

Erysimum bonannianum C.Presl

Erysimum brulloi G.Ferro

Erysimum burnatii G.Vidal

Erysimum collisparsum Jord.

Erysimum crassistylum C.Presl subsp. crassistylum

Erysimum crassistylum C.Presl subsp. garganicum Peccenini \& Polatschek

Erysimum crassistylum C.Presl subsp. verresianum Peccenini \& Polatschek

Erysimum etnense Jord.

Erysimum etruscum Peccenini \& Polatschek

Erysimum insubricum Peccenini \& Polatschek

Erysimum jugicola Jord.

Erysimum ligusticum Peccenini \& Polatschek

Erysimum majellense Polatschek

Erysimum maremmanum Peccenini \& Polatschek

Erysimum metlesicsii Polatschek

Erysimum montis-argentarii Peccenini \& Polatschek

Erysimum odoratum Ehrh.

Erysimum pignattii Peccenini \& Polatschek

Erysimum pseudorhaeticum Polatschek

Erysimum rhaeticum (Schleich. ex Hornem.) DC.

Erysimum sylvestre (Crantz) Scop.

Erysimum virgatum Roth

Fibigia clypeata (L.) Medik. subsp. clypeata

Fourraea alpina (L.) Greuter \& Burdet

Hesperis inodora $\mathrm{L}$.

Hesperis laciniata All. subsp. cupaniana (Guss.) Giardina \& Raimondo

Hesperis Iaciniata All. subsp. Iaciniata

Hesperis matronalis L. subsp. matronalis

Hesperis matronalis L. subsp. nivea (Baumg.) E.P.Perrier

Hirschfeldia incana (L.) Lagr.-Foss. subsp. incana

Hormathophylla ligustica (Breistr.) Španiel, Al-Shehbaz, D.A.German \& Marhold

Hornungia alpina (L.) O.Appel subsp. alpina

Hornungia alpina (L.) O.Appel subsp. austroalpina (Trpin) O.Appel

Hornungia alpina (L.) O.Appel subsp. brevicaulis (Spreng.) O.Appel

Hornungia pauciflora (W.D.J.Koch) Soldano, F.Conti, Banfi \& Galasso

Hornungia petraea (L.) Rchb. subsp. petraea

Hornungia procumbens (L.) Hayek

Hugueninia tanacetifolia (L.) Rchb. subsp. tanacetifolia

Iberis aurosica Chaix subsp. nana (All.) Moreno

Iberis linifolia L. subsp. linifolia

Iberis linifolia L. subsp. stricta (Jord.) P.Fourn.

Iberis pinnata $\mathrm{L}$.

Iberis saxatilis L. subsp. saxatilis

Iberis semperflorens $L$.

Iberis sempervirens $\mathrm{L}$.

Iberis umbellata $L$.

Iberis violacea $\mathrm{R}$. Br.

Ihsanalshehbazia granatensis (Boiss. \& Reut.) Tahir Ali \& Thines

Ionopsidium albiflorum Durieu

Ionopsidium savianum (Caruel) Arcang.

Isatis apennina Ten. ex Grande

$\mathrm{T} \quad$ Isatis praecox Kit. ex Tratt.

ET Isatis raimondoi Di Grist., Scafidi \& Domina

Kernera saxatilis (L.) Sweet subsp. saxatilis

Lepidium campestre (L.) R.Br.

Lepidium coronopus (L.) Al-Shehbaz

Lepidium draba L. subsp. draba

Lepidium graminifolium L. subsp. graminifolium

Lepidium hirtum (L.) Sm. subsp. hirtum 
Lepidium hirtum (L.) Sm. subsp. nebrodense (Raf.) Thell.

Lepidium Iatifolium $\mathrm{L}$.

Lepidium ruderale $\mathrm{L}$.

Lepidium villarsii Gren. \& Godr. subsp. villarsii

Lobularia libyca (Viv.) Meisn.

Lobularia maritima (L.) Desv.

Lunaria annua $\mathrm{L}$.

Lunaria rediviva $\mathrm{L}$.

Malcolmia flexuosa (Sm.) Sm.

Malcolmia maritima (L.) W.T.Aiton

Malcolmia orsiniana (Ten.) Ten. subsp. orsiniana

Marcus-kochia littorea (L.) Al-Shehbaz

Marcus-kochia ramosissima (Desf.) Al-Shehbaz

Maresia nana (DC.) Batt.

Matthiola fruticulosa (L.) Maire subsp. coronopifolia (Sm.) Giardina \& Raimondo

Matthiola fruticulosa (L.) Maire subsp. fruticulosa

Matthiola fruticulosa (L.) Maire subsp. valesiaca (Boiss.) P.W.Ball

Matthiola incana (L.) W.T.Aiton subsp. incana

E Matthiola incana (L.) W.T.Aiton subsp. pulchella (Conti) Greuter \& Burdet

E Matthiola incana (L.) W.T.Aiton subsp. rupestris (Raf.) Nyman

Matthiola sinuata (L.) W.T.Aiton

Matthiola tricuspidata (L.) R.Br.

Microthlaspi perfoliatum (L.) F.K.Mey.

Moricandia arvensis (L.) DC.

Moricandia longirostris Pomel

E Morisia monanthos (Viv.) Asch.

Murbeckiella pinnatifida (Lam.) Rothm.

Murbeckiella zanonii (Ball) Rothm.

Myagrum perfoliatum $\mathrm{L}$.

Nasturtium microphyllum (Boenn.) Rchb.

Nasturtium officinale R.Br.

Nasturtium sterile (Airy Shaw) Oefelein

Neslia paniculata (L.) Desv. subsp. paniculata

Neslia paniculata (L.) Desv. subsp. thracica (Velen.) Bornm.

Noccaea brachypetala (Jord.) F.K.Mey.

Noccaea brevistyla (DC.) Steud.

Noccaea caerulescens (J.Presl \& C.Presl) F.K.Mey.

Noccaea cepaeifolia (Wulfen) Rchb.

Noccaea corymbosa (J.Gay) F.K.Mey.

Noccaea limosellifolia (Reut. ex Burnat) F.K.Mey.

Noccaea minima (Ard.) F.K.Mey.

Noccaea montana (L.) F.K.Mey.

Noccaea praecox (Wulfen) F.K.Mey.

Noccaea rotundifolia (L.) Moench

DD Noccaea salisii (Brügger) F.K.Mey.

E Noccaea stylosa (Ten.) Rchb.

Noccaea sylvia (Gaudin) F.K.Mey.

E Noccaea torreana (Ten.) Bartolucci, Galasso \& Peruzzi

Noccaea virens (Jord.) F.K.Mey.

Odontarrhena alpestris (L.) Ledeb.

E Odontarrhena argentea (All.) Ledeb.

E Odontarrhena bertolonii (Desv.) Jord. \& Fourr.

E Odontarrhena nebrodensis (Tineo) L.Cecchi \& Selvi subsp. nebrodensis

E Odontarrhena tavolarae (Briq.) L.Cecchi \& Selvi

Petrocallis pyrenaica (L.) R.Br.

E Phyllolepidum rupestre (Sweet) Trinajstić

Pseudoturritis turrita (L.) Al-Shehbaz

Raphanus raphanistrum L. subsp. Iandra (Moretti ex DC.) Bonnier \& Layens

Raphanus raphanistrum $L$. subsp. raphanistrum

Rapistrum rugosum (L.) All.

E Rhizobotrya alpina Tausch - Note: For a history of this name, see Villani et al. (2015).

Rorippa amphibia (L.) Besser

Rorippa islandica (Oeder) Borbás

Rorippa lippizensis (Wulfen) Rchb.

Rorippa palustris (L.) Besser

Rorippa prostrata (J.P.Bergeret) Schinz \& Thell.

Rorippa pyrenaica (All.) Rchb.

Rorippa sylvestris (L.) Besser subsp. sylvestris

Sinapis alba L. subsp. alba

T Sinapis alba L. subsp. dissecta (Lag.) Bonnier

T Sinapis alba L. subsp. mairei (H.Lindb.) Maire

Sinapis arvensis L. subsp. arvensis

Sinapis pubescens $L$. subsp. pubescens

E Sisymbrella dentata (L.) O.E.Schulz - Note: For the typification of this name, see Peruzzi et al. (2013c). Sisymbrium altissimum $\mathrm{L}$.

Sisymbrium austriacum Jacq. subsp. austriacum

Sisymbrium erysimoides Desf.

Sisymbrium irio L. 
Sisymbrium officinale (L.) Scop.

Sisymbrium orientale $\mathrm{L}$.

Sisymbrium polyceratium L.

Sisymbrium strictissimum $\mathrm{L}$.

Strigosella africana (L.) Botsch.

Succowia balearica (L.) Medik.

Teesdalia coronopifolia (J.P.Bergeret) Thell.

Teesdalia nudicaulis (L.) R.Br.

Thlaspi alliaceum $\mathrm{L}$.

Thlaspi arvense $\mathrm{L}$.

Turritis glabra L.

Loranthaceae

Loranthus europaeus Jacq.

Santalaceae

Arceuthobium oxycedri (DC.) M.Bieb.

Osyris alba L.

Thesium alpinum $\mathrm{L}$.

Thesium arvense Horv.

Thesium bavarum Schrank

Thesium ebracteatum Hayne

Thesium humifusum DC

Thesium humile Vahl

Thesium italicum A.DC

Thesium linophyllon $L$.

Thesium parnassi A.DC

Thesium pyrenaicum Pourr. subsp. grandiflorum (DC.) Hendrych

Thesium pyrenaicum Pourr. subsp. pyrenaicum

Thesium rostratum Mert. \& W.D.J.Koch

E Thesium sommieri Hendrych

Viscum album L. subsp. abietis (Wiesb.) Abrom.

Viscum album L. subsp. album

Viscum album L. subsp. austriacum (Wiesb.) Vollm.

Plumbaginaceae

Taxonomic references: Armeria Willd. (Arrigoni 2015).

Armeria alpina Willd.

ET Armeria arenaria (Pers.) Schult. subsp. apennina Arrigoni

Armeria arenaria (Pers.) Schult. subsp. arenaria

ET Armeria arenaria (Pers.) Schult. subsp. marginata (Levier) Arrigoni

Armeria aspromontana Brullo, Scelsi \& Spamp.

Armeria brutia Brullo, Gangale \& Uzunov

Armeria denticulata (Bertol.) DC.

Armeria garganica Arrigoni

Armeria gracilis Ten. subsp. gracilis

Armeria gracilis Ten. subsp. majellensis (Boiss.) Arrigoni

Armeria gussonei Boiss.

Armeria helodes F.Martini \& Poldini

Armeria macropoda Boiss.

Armeria morisii Boiss.

Armeria nebrodensis (Guss.) Boiss.

Armeria pungens (Link) Hoffmanns. \& Link

Armeria sardoa Spreng. subsp. genargentea Arrigoni

Armeria sardoa Spreng. subsp. sardoa

Armeria saviana Selvi

Armeria sulcitana Arrigoni

Goniolimon italicum Tammaro, Pignatti \& Frizzi - Note: For the biology and distribution of this species, see Conti et al. (2008) and Morretti et al. (2015).

Limoniastrum monopetalum (L.) Boiss.

Limonium acutifolium (Rchb.) Salmon subsp. acutifolium

Limonium acutifolium (Rchb.) Salmon subsp. bosanum (Arrigoni \& Diana) Arrigoni

Limonium acutifolium (Rchb.) Salmon subsp. cornusianum (Arrigoni \& Diana) Arrigoni

Limonium acutifolium (Rchb.) Salmon subsp. nymphaeum (Erben) Arrigoni

Limonium acutifolium (Rchb.) Salmon subsp. tenuifolium (Bertol. ex Moris) Arrigoni

Limonium acutifolium (Rchb.) Salmon subsp. tharrosianum (Arrigoni \& Diana) Arrigoni

Limonium aegusae Brullo

Limonium albidum (Guss.) Pignatti

Limonium algusae (Brullo) Greuter

Limonium ampuriense Arrigoni \& Diana

Limonium amynclaeum Pignatti

Limonium apulum Brullo

Limonium avei (De Not.) Brullo \& Erben

Limonium bellidifolium (Gouan) Dumort.

Limonium bocconei (Lojac.) Litard.

Limonium brutium Brullo

Limonium calabrum Brullo

Limonium calcarae (Tod. ex Janka.) Pignatti 
E Limonium capitis-eliae Erben

E Limonium capitis-marci Arrigoni \& Diana

E Limonium caprariae Rizzotto

E Limonium carisae Erben

E Limonium catanense (Tineo ex Lojac.) Brullo - Note: Extinct (Rossi et al. 2013).

E Limonium catanzaroi Brullo

Limonium circaei Pignatti

Limonium contortirameum (Mabille) Erben

Limonium cophanense C.Brullo, Brullo, Cambria, Giusso \& Ilardi

Limonium coralliforme Alf.Mayer

Limonium cordatum (L.) Mill.

E Limonium cosyrense (Guss.) Kuntze

E Limonium cumanum (Ten.) Kuntze

E Limonium cunicularium Arrigoni \& Diana

Limonium densiflorum (Guss.) Kuntze

Limonium densissimum (Pignatti) Pignatti

E Limonium diomedeum Brullo

Limonium divaricatum (Rouy) Brullo

E Limonium dolcheri Pignatti

E Limonium doriae (Sommier) Pignatti

E Limonium dubium (Andrews ex Guss.) Litard.

Limonium echioides (L.) Mill.

E Limonium etruscum Arrigoni \& Rizzotto

E Limonium flagellare (Lojac.) Brullo

Limonium furnarii Brullo

Limonium gallurense Arrigoni \& Diana

Limonium glomeratum (Tausch) Erben

Limonium gorgonae Pignatti

Limonium hermaeum (Pignatti) Pignatti

Limonium hyblaeum Brullo

Limonium ilvae Pignatti

Limonium inarimense (Guss.) Pignatti

Limonium insulare (Bég. \& Landi) Arrigoni \& Diana

Limonium intermedium (Guss.) Brullo - Note: Extinct in the wild. At present, some individuals of L. intermedium survive in the Botanical Garden of Catania, grown from seeds sampled in the field about 40 years ago (Rossi et al. 2013; Domina et al. 2015).

Limonium ionicum Brullo

Limonium jankae (Lojac.) Giardina \& Raimondo

Limonium japygicum (E.Groves) Pignatti ex Pignatti, Galasso \& Nicolella

Limonium Iacinium Arrigoni

Limonium laetum (Nyman) Pignatti

Limonium lausianum Pignatti

Limonium lilybaeum Brullo

Limonium Iojaconoi Brullo

Limonium lopadusanum Brullo

Limonium malfatanicum Erben

Limonium mazarae Pignatti

Limonium melancholicum Brullo, Marcenò \& S.Romano

Limonium merxmuelleri Erben subsp. merxmuelleri

Limonium merxmuelleri Erben subsp. oristanum (Alf.Mayer) Arrigoni

Limonium merxmuelleri Erben subsp. sulcitanum (Arrigoni) Arrigoni

Limonium merxmuelleri Erben subsp. tigulianum (Arrigoni \& Diana) Arrigoni

Limonium minutiflorum (Guss.) Kuntze

Limonium morisianum Arrigoni

Limonium multiforme Pignatti

Limonium multifurcatum Erben

Limonium narbonense Mill.

Limonium optimae Raimondo

Limonium opulentum (Lojac.) Brullo

Limonium pachynense Brullo

Limonium pandatariae Pignatti

Limonium panormitanum (Tod.) Pignatti

Limonium parvifolium (Tineo) Pignatti

Limonium pavonianum Brullo

Limonium peucetium Pignatti - Note: Possibly extinct (Rossi et al. 2013).

Limonium planesiae Pignatti

Limonium poimenum Ilardi, Brullo, D.Cusimano \& Giusso

Limonium pontium Pignatti

Limonium ponzoi (Fiori) Brullo

Limonium protohermaeum Arrigoni \& Diana

Limonium pseudolaetum Arrigoni \& Diana

Limonium pulviniforme Arrigoni \& Diana

Limonium racemosum (Lojac.) Diana

Limonium ramosissimum (Poir.) Maire

Limonium remotispiculum (Lacaita) Pignatti

Limonium retirameum Greuter \& Burdet subsp. caralitanum Arrigoni

Limonium retirameum Greuter \& Burdet subsp. retirameum

Limonium secundirameum (Lojac.) Brullo

Limonium selinuntinum Brullo 
Limonium sibthorpianum (Guss.) Kuntze

Limonium sinuatum (L.) Mill.

E Limonium sommierianum (Fiori) Arrigoni

E Limonium strictissimum (Salzm.) Arrigoni

Limonium syracusanum Brullo

Limonium tauromenitanum Brullo

Limonium tenoreanum (Guss.) Pignatti - Note: For the taxonomy and distribution of this species, see lamonico et al. (2017).

Limonium tenuiculum (Tineo ex Guss.) Pignatti

Limonium tibulatium Pignatti

Limonium tineoi (Lojac.) Giardina \& Raimondo

Limonium todaroanum Raimondo \& Pignatti

Limonium tyrrhenicum Arrigoni \& Diana

Limonium ursanum Erben

Limonium usticanum Giardina \& Raimondo

Limonium viniolae Arrigoni \& Diana

Limonium virgatum (Willd.) Fourr.

Myriolimon ferulaceum (L.) Lledó, Erben \& M.B.Crespo

Plumbago europaea $\mathrm{L}$.

Polygonaceae

Taxonomic references: Persicaria (L.) Mill. (Galasso et al. 2014).

Bistorta officinalis Delarbre

Bistorta vivipara (L.) Delarbre

Fallopia convolvulus (L.) Á.Löve

Fallopia dumetorum (L.) Holub

Koenigia alpina (All.) T.M.Schust. \& Reveal - Note: According to Schuster et al. (2015), the genus Aconogonon (Meisn.) Rchb. is nested within Koenigia L.

Oxyria digyna (L.) Hill

Persicaria amphibia (L.) Delarbre

Persicaria decipiens (R.Br.) K.L.Wilson

Persicaria dubia (Stein.) Fourr.

Persicaria hydropiper (L.) Delarbre

DD Persicaria lapathifolia (L.) Delarbre subsp. brittingeri (Opiz) Soják

Persicaria lapathifolia (L.) Delarbre subsp. Iapathifolia

Persicaria lapathifolia (L.) Delarbre subsp. pallida (With.) Á.Löve

Persicaria maculosa Gray

Persicaria minor (Huds.) Opiz

Polygonum arenarium Waldst. \& Kit. subsp. pulchellum (Loisel.) Thell.

Polygonum arenastrum Boreau

Polygonum aviculare L. subsp. aviculare

Polygonum bellardii All.

Polygonum equisetiforme $\mathrm{Sm}$.

Polygonum maritimum $\mathrm{L}$.

Polygonum robertii Loisel.

Polygonum romanum Jaca.

Polygonum rurivagum Jord. ex Boreau

E Polygonum scoparium Req. ex Loisel.

E Polygonum tenorei C.Presl

Rumex acetosa L. subsp. acetosa

Rumex acetosella L. subsp. acetosella

Rumex acetosella L. subsp. acetoselloides (Balansa) Den Nijs

Rumex acetosella L. subsp. multifidus (L.) Schübl. \& G.Martens

Rumex acetosella L. subsp. pyrenaicus (Pourr. ex Lapeyr.) Akeroyd

Rumex alpinus L.

Rumex aquaticus $\mathrm{L}$.

Rumex arifolius All.

Rumex bucephalophorus L. subsp. bucephalophorus

Rumex bucephalophorus L. subsp. gallicus (Steinh.) Rech.f.

Rumex confertus Willd.

Rumex conglomeratus Murray

Rumex crispus L.

C Rumex cristatus DC.

C Rumex dentatus $L$.

Rumex hydrolapathum Huds.

Rumex intermedius DC

Rumex maritimus $\mathrm{L}$.

Rumex nebroides Campd.

Rumex nepalensis Spreng.

Rumex obtusifolius L. subsp. obtusifolius

Rumex obtusifolius L. subsp. sylvestris (Wallr. ex Becker) Čelak.

Rumex obtusifolius L. subsp. transiens (Simonk.) Rech.f.

Rumex palustris $\mathrm{Sm}$

Rumex pulcher L. subsp. pulcher

E Rumex pulcher L. subsp. suffocatus (Moris ex Bertol.) Nyman

Rumex pulcher L. subsp. woodsii (De Not.) Arcang.

Rumex sanguineus $L$.

ET Rumex scutatus L. subsp. aetnensis (C.Presl) Cif. \& Giacom.

ET Rumex scutatus L. subsp. glaucescens (Guss.) Brullo, Scelsi \& Spamp.

Rumex scutatus L. subsp. scutatus 
Rumex spinosus L. - Note: According to Schuster et al. (2015), the genus Emex Neck. ex Campd. is nested within Rumex L.

Rumex thyrsoides Desf.

Rumex tuberosus L. subsp. tuberosus

Droseraceae

Aldrovanda vesiculosa L. - Note: Extinct in Italy (Rossi et al. 2013).

Drosera anglica Huds.

Drosera intermedia Hayne

Drosera rotundifolia L.

Frankeniaceae

Frankenia hirsuta $\mathrm{L}$.

Frankenia laevis L. subsp. laevis

Frankenia pulverulenta $\mathrm{L}$. subsp. pulverulenta

Tamaricaceae

Myricaria germanica (L.) Desv.

Reaumuria vermiculata $L$.

Tamarix africana Poir.

Tamarix arborea Ehrenb. ex Bunge

Tamarix canariensis Willd.

Tamarix dalmatica Baum

Tamarix gallica $\mathrm{L}$.

Tamarix hampeana Boiss. \& Heldr.

Tamarix tetragyna Ehrenb.

Caryophyllaceae

Taxonomic references: Polycarpon tetraphyllum (L.) L. (lamonico and Domina 2015); Spergula L. and Spergularia (Pers.) J.Presl \& C.Presl (Kool and Thulin 2017); Cherleria L., Facchinia Rchb., Sabulina Rchb., Mcneillia Dillenb. \& Kadereit, and Minuartia L. s.str. (Bartolucci et al. 2014a; Dillenberger and Kadereit 2014, 2015; lamonico 2016b; Moore and Dillenberger 2017).

Arenaria aggregata (L.) Loisel. subsp. aggregata

Arenaria balearica $\mathrm{L}$.

E Arenaria bertolonii Fiori - Note: For the taxonomy and distribution of this species, see lamonico (2013a).

Arenaria biflora L.

DD Arenaria ciliata L. subsp. bernensis Favarger

Arenaria ciliata L. subsp. ciliata

Arenaria gothica Fr. subsp. moehringioides (Murr) M.B.Wyse Jacks. \& J.Parn.

Arenaria grandiflora $\mathrm{L}$. subsp. grandiflora

E Arenaria huteri A.Kern.

Arenaria leptoclados (Rchb.) Guss. subsp. leptoclados

Arenaria marschlinsii W.D.J.Koch

Arenaria montana L. subsp. intricata (Ser.) Pau

Arenaria serpyllifolia L. subsp. serpyllifolia

Atocion armeria (L.) Raf.

Atocion rupestre (L.) Oxelman

Bufonia paniculata Dubois ex Delarbre

Bufonia tenuifolia L.

Cerastium alpinum L.

E Cerastium apuanum Parl.

Cerastium arvense $L$. subsp. arvense

Cerastium arvense L. subsp. strictum Gaudin

Cerastium arvense L. subsp. suffruticosum (L.) Ces.

Cerastium boissierianum Greuter \& Burdet

Cerastium brachypetalum Desp. ex Pers. subsp. brachypetalum

Cerastium brachypetalum Desp. ex Pers. subsp. roeseri (Boiss. \& Heldr.) Nyman

Cerastium brachypetalum Desp. ex Pers. subsp. tenoreanum (Ser.) Soó

Cerastium carinthiacum Vest subsp. austroalpinum (Kunz) Janch.

Cerastium carinthiacum Vest subsp. carinthiacum

Cerastium cerastoides (L.) Britton

Cerastium diffusum Pers. subsp. diffusum

Cerastium diffusum Pers. subsp. gussonei (Lojac.) P.D.Sell \& Whitehead

Cerastium dubium (Bastard) Guépin

Cerastium fontanum Baumg.

Cerastium glomeratum Thuill.

Cerastium glutinosum $\mathrm{Fr}$

E Cerastium granulatum (Huter, Porta \& Rigo) Porta

Cerastium holosteoides $\mathrm{Fr}$.

E Cerastium lacaitae Barberis, Bechi \& Miceli

Cerastium latifolium $\mathrm{L}$.

Cerastium ligusticum Viv.

Cerastium lineare All.

Cerastium lucorum (Schur) Möschl

$E$

Cerastium palustre Moris

Cerastium pedunculatum Gaudin

Cerastium pentandrum $\mathrm{L}$.

E Cerastium pospichalii Soldano \& F.Conti

Cerastium pumilum Curtis

Cerastium scaranoi Ten. 
Cerastium semidecandrum $\mathrm{L}$.

Cerastium siculum Guss.

Cerastium subtriflorum (Rchb.) Pacher

Cerastium supramontanum Arrigon

Cerastium sylvaticum Waldst. \& Kit.

E Cerastium thomasii Ten.

E Cerastium tomentosum $\mathrm{L}$.

Cerastium uniflorum Clairv.

Cerastium utriense Barberis

Chaetonychia cymosa (L.) Sweet

Cherleria biflora (L.) A.J.Moore \& Dillenb.

Cherleria capillacea (All.) A.J.Moore \& Dillenb.

Cherleria Iaricifolia (L.) lamonico subsp. Iaricifolia

ET Cherleria Iaricifolia (L.) lamonico subsp. ophiolitica (Pignatti) lamonico

Cherleria sedoides L.

Corrigiola litoralis L. subsp. litoralis

Corrigiola telephiifolia Pourr.

Dianthus armeria L. subsp. armeria

Dianthus arrostii C.Presl

Dianthus balbisii Ser. subsp. balbisii

T Dianthus balbisii Ser. subsp. liburnicus (Bartl.) Pignatti

Dianthus barbatus L. subsp. barbatus

T Dianthus barbatus L. subsp. compactus (Kit.) Heuff.

E Dianthus borbonicus Brullo, C.Brullo, Colombo, Giusso, Ilardi \& R.Perrone

E Dianthus brachycalyx A.Huet \& É.Huet ex Bacch., Brullo, Casti \& Giusso

ET Dianthus brutius Brullo, Scelsi \& Spamp. subsp. brutius

ET Dianthus brutius Brullo, Scelsi \& Spamp. subsp. pentadactyli Brullo, Scelsi \& Spamp.

E Dianthus busambrae Soldano \& F.Conti

T Dianthus carthusianorum L. subsp. atrorubens (All.) Pers.

Dianthus carthusianorum L. subsp. carthusianorum

Dianthus carthusianorum L. subsp. tenorei (Lacaita) Pignatti

Dianthus ciliatus Guss. subsp. ciliatus

E Dianthus cyatophorus Moris subsp. cyatophorus

E Dianthus cyatophorus Moris subsp. minae (Mazzola, Raimondo, llardi) Raimondo

Dianthus deltoides L. subsp. deltoides

E Dianthus furcatus Balb. subsp. dissimilis (Burnat) Pignatti

Dianthus furcatus Balb. subsp. furcatus

E Dianthus furcatus Balb. subsp. lereschii (Burnat) Pignatti

E Dianthus gasparrinii Guss.

E Dianthus genargenteus Bacch., Brullo, Casti \& Giusso

Dianthus glacialis Haenke subsp. glacialis

Dianthus graminifolius C.Pres

E Dianthus guliae Janka

Dianthus hyssopifolius L.

E Dianthus ichnusae Bacch., Brullo, Casti \& Giusso subsp. ichnusae

E Dianthus ichnusae Bacch., Brullo, Casti \& Giusso subsp. toddei Bacch., Brullo, Casti \& Giusso

E Dianthus insularis Bacch., Brullo, Casti \& Giusso

E Dianthus japigicus Bianco \& Brullo

Dianthus longicaulis Ten.

E Dianthus morisianus Vals.

E Dianthus mossanus Bacch. \& Brullo

E Dianthus oliastrae Bacch., Brullo, Casti \& Giusso

Dianthus pavonius Tausch

E Dianthus rupicola Biv. subsp. aeolicus (Lojac.) Brullo \& Miniss.

E Dianthus rupicola Biv. subsp. Iopadusanus Brullo \& Miniss.

Dianthus rupicola Biv. subsp. rupicola

Dianthus sanguineus Vis.

E Dianthus sardous Bacch., Brullo, Casti \& Giusso

Dianthus seguieri Vill. subsp. seguieri

Dianthus siculus C.Presl

Dianthus sternbergii Sieber ex Capelli

Dianthus superbus L. subsp. alpestris (R.Uechtr.) Kablík. ex Čelak.

Dianthus superbus $L$. subsp. superbus

Dianthus sylvestris Wulfen subsp. sylvestris

T Dianthus sylvestris Wulfen subsp. tergestinus (Rchb.) Hayek

E Dianthus tarentinus Lacaita

Dianthus tripunctatus Sm.

E Dianthus virgatus Pasq.

ET Dianthus vulturius Guss. \& Ten. subsp. aspromontanus Brullo, Scelsi \& Spamp.

E Dianthus vulturius Guss. \& Ten. subsp. vulturius

Drypis spinosa L. subsp. jacquiniana Wettst. \& Murb.

Drypis spinosa L. subsp. spinosa

Eudianthe coeli-rosa (L.) Endl.

Eudianthe laeta Rchb. ex Willk.

Facchinia cherlerioides (Sieber) Dillenb. \& Kadereit subsp. cherlerioides

E Facchinia grignensis (Rchb.) Dillenb. \& Kadereit

Facchinia herniarioides (Rion) Dillenb. \& Kadereit

Facchinia lanceolata (All.) Rchb. 
Facchinia rupestris (Scop.) Dillenb. \& Kadereit

Gypsophila arrostii Guss. subsp. arrostii

E Gypsophila papillosa Porta

Gypsophila repens L.

Heliosperma alpestre (Jacq.) Griseb.

Heliosperma pusillum (Waldst. \& Kit.) Rchb. subsp. pudibundum (Hoffmanns.) Gutermann

Heliosperma pusillum (Waldst. \& Kit.) Rchb. subsp. pusillum

Herniaria alpina Chaix

E Herniaria bornmuelleri Chaudhri

Herniaria cinerea DC.

E Herniaria fontanesii Gay subsp. empedocleana (Lojac.) Brullo - Note: Extinct (Rossi et al. 2013).

Herniaria glabra L. subsp. glabra

Herniaria glabra L. subsp. nebrodensis Jan ex Nyman

E Herniaria hirsuta L. subsp. aprutia Chaudhri

Herniaria hirsuta L. subsp. hirsuta

Herniaria incana Lam.

E Herniaria litardierei (Gamisans) Greuter \& Burdet

Herniaria permixta Guss.

Holosteum umbellatum L. subsp. glutinosum (M.Bieb.) Nyman

Holosteum umbellatum L. subsp. umbellatum

Illecebrum verticillatum $L$.

Loeflingia hispanica $\mathrm{L}$.

Lychnis coronaria (L.) Desr.

Lychnis flos-cuculi L. subsp. flos-cuculi

Lychnis flos-jovis (L.) Desr.

Mcneillia graminifolia (Ard.) Dillenb. \& Kadereit subsp. clandestina (Port.) Dillenb. \& Kadereit

E Mcneillia graminifolia (Ard.) Dillenb. \& Kadereit subsp. graminifolia

E Mcneillia graminifolia (Ard.) Dillenb. \& Kadereit subsp. rosanoi (Ten.) F.Conti, Bartolucci, lamonico \& Del Guacchio

E Mcneillia moraldoi (F.Conti) Dillenb. \& Kadereit

E Minuartia glomerata (M.Bieb.) Degen subsp. trichocalycina (Ten. \& Guss.) F.Conti

Minuartia recurva (All.) Schinz \& Thell. subsp. condensata (C.Presl) Greuter \& Burdet

Minuartia recurva (All.) Schinz \& Thell. subsp. recurva

Minuartia rostrata (Pers.) Rchb.

Minuartia rubra (Scop.) McNeill

Moehringia argenteria Casazza \& Minuto

Moehringia bavarica (L.) Gren.

Moehringia ciliata (Scop.) Dalla Torre

E Moehringia concarenae F.Fen. \& F.Martini

E Moehringia dielsiana Mattf.

E Moehringia glaucovirens Bertol.

E Moehringia insubrica Degen

Moehringia intermedia (Loisel.) Panizzi

Moehringia lebrunii Merxm

E Moehringia markgrafii Merxm. \& Gutermann

Moehringia muscosa $\mathrm{L}$.

E Moehringia papulosa Bertol.

Moehringia pentandra Gay

Moehringia sedoides (Pers.) Loisel.

Moehringia tommasinii Marches.

Moehringia trinervia (L.) Clairv.

Moehringia villosa (Wulfen) Fenzl subsp. glabrescens (Rchb. ex Nyman) Starm.

Moenchia erecta (L.) G.Gaertn., B.Mey. \& Scherb. subsp. erecta

Moenchia erecta (L.) G.Gaertn., B.Mey. \& Scherb. subsp. octandra (Ziz ex Mert. \& W.D.J.Koch) Gürke ex Cout.

Moenchia mantica (L.) Bartl. subsp. mantica

Paronychia arabica (L.) DC. subsp. Iongiseta Batt.

Paronychia argentea Lam.

Paronychia capitata (L.) Lam. subsp. capitata

Paronychia echinulata Chater

Paronychia kapela (Hacq.) A.Kern. subsp. kapela

Paronychia kapela (Hacq.) A.Kern. subsp. serpyllifolia (Chaix) Graebn.

Paronychia polygonifolia (Vill.) DC.

Petrorhagia dubia (Raf.) G.López \& Romo

Petrorhagia illyrica (Ard.) P.W.Ball \& Heywood subsp. haynaldiana (F.N.Williams) P.W.Ball \& Heywood

Petrorhagia nanteuilii (Burnat) P.W.Ball \& Heywood

Petrorhagia prolifera (L.) P.W.Ball \& Heywood

T Petrorhagia saxifraga (L.) Link subsp. gasparrinii (Guss.) Greuter \& Burdet

Petrorhagia saxifraga (L.) Link subsp. saxifraga

Polycarpon tetraphyllum (L.) L. subsp. alsinifolium (Biv.) Ball

Polycarpon tetraphyllum (L.) L. subsp. diphyllum (Cav.) O.Bolòs \& Font Quer

Polycarpon tetraphyllum (L.) L. subsp. polycarpoides (Biv.) lamonico

Polycarpon tetraphyllum (L.) L. subsp. tetraphyllum

Psammophiliella muralis (L.) Ikonn.

Pseudostellaria europaea Schaeftl.

Rhodalsine geniculata (Poir.) F.N.Williams

Sabulina attica (Boiss. \& Sprun.) Dillenb. \& Kadereit

Sabulina austriaca (Jacq.) Rchb.

T Sabulina glaucina (Dvoráková) Dillenb. \& Kadereit

Sabulina mediterranea (Ledeb. ex Link) Rchb. 
Sabulina tenuifolia (L.) Rchb. subsp. tenuifolia - Note: For the nomenclature of this species, see lamonico (2014b).

Sabulina tenuifolia (L.) Rchb. subsp. vaillantiana (DC.) Dillenb. \& Kadereit

Sabulina verna (L.) Rchb. subsp. grandiflora (C.Presl) Dillenb. \& Kadereit

Sabulina verna (L.) Rchb. subsp. verna

Sabulina villarsii (Balb.) Rchb.

Sabulina viscosa (Schreb.) Rchb.

Sagina alexandrae lamonico - Note: For the nomenclature of this species, see lamonico (2016c).

Sagina apetala Ard. subsp. apetala

Sagina glabra (Willd.) Fenzl

Sagina maritima Don

Sagina micropetala Rauschert

Sagina nodosa (L.) Fenzl

Sagina pilifera (DC.) Fenzl

Sagina procumbens $\mathrm{L}$.

E Sagina revelierei Jord. \& Fourr.

Sagina saginoides (L.) H.Karst. subsp. saginoides

Saponaria bellidifolia Sm.

Saponaria calabrica Guss.

Saponaria lutea $\mathrm{L}$.

E Saponaria ocymoides L. subsp. alsinoides (Viv.) Arcang.

Saponaria ocymoides L. subsp. ocymoides

Saponaria officinalis $\mathrm{L}$.

Saponaria pumila Janch.

Saponaria sicula Raf.

E Scleranthus aetnensis Strobl

Scleranthus annuus L.

Scleranthus perennis L. subsp. burnatii (Briq.) P.D.Sell

Scleranthus perennis $L$. subsp. dichotomus (Schur) Nyman

Scleranthus perennis L. subsp. marginatus (Guss.) Nyman

Scleranthus perennis L. subsp. perennis

E Scleranthus perennis L. subsp. stroblii (Rchb. ex Strobl) Giardina \& Raimondo

E Scleranthus perennis L. subsp. vulcanicus (Strobl) Bég.

Scleranthus polycarpos $\mathrm{L}$.

Scleranthus uncinatus Schur

Scleranthus verticillatus Tausch

Silene acaulis (L.) Jacq. subsp. acaulis

$\mathrm{T} \quad$ Silene acaulis (L.) Jacq. subsp. bryoides (Jord.) Nyman

Silene acaulis (L.) Jacq. subsp. cenisia Vierh.

Silene apetala Willd.

Silene arghireica Vals.

Silene baccifera (L.) Durande

Silene badaroi Breistr.

E Silene beguinotii Vals.

Silene behen $\mathrm{L}$.

Silene bellidifolia Jacq

E Silene calabra Brullo, Scelsi \& Spamp.

Silene campanula Pers.

Silene canescens Ten.

Silene catholica (L.) W.T.Aiton

E Silene cattariniana Ferrarini \& Cecchi

Silene ciliata Pourr. subsp. graefferi (Guss.) Nyman

Silene colorata Poir.

Silene conica L.

Silene cordifolia All.

E Silene crassiuscula Brullo, C.Brullo, Cambria, Bacch., Giusso \& Ilardi

Silene cretica L.

Silene dichotoma Ehrh.

Silene dioica (L.) Clairv.

Silene diversifolia Otth

E Silene echinata Otth

E Silene elisabethae Jan

Silene fruticosa $\mathrm{L}$.

Silene fuscata Brot

Silene gallica $\mathrm{L}$.

Silene gallinyi Heuff. ex Rchb.

E Silene giraldii Guss.

Silene hayekiana Hand.-Mazz. \& Janch.

E Silene hicesiae Brullo \& Signor.

E Silene ichnusae Brullo, De Marco \& De Marco f.

Silene inaperta $\mathrm{L}$.

Silene italica (L.) Pers. subsp. italica

E Silene italica (L.) Pers. subsp. sicula (Ucria) Jeanm.

E Silene kemoniana C.Brullo, Brullo, Giusso, Ilardi \& Sciandr.

E Silene lanuginosa Bertol.

Silene Iatifolia Poir. - Note: Silene latifolia Poir. subsp. alba (Mill.) Greuter \& Burdet [= S. pratensis (Rafn) Godr.] is included here.

Silene linicola C.C.Gmel.

E Silene martinolii Bocchieri \& Mulas

Silene minae Strobl 
E Silene morisiana Bég. \& Ravano

Silene multicaulis Guss. subsp. multicaulis

Silene muscipula L. subsp. muscipula

Silene mutabilis L. - Note: For the nomenclature of this species, see Peruzzi et al. (2014b).

E Silene nefelites C.Brullo, Brullo, Giusso \& llardi

Silene nemoralis Waldst. \& Kit.

Silene niceensis All.

Silene noctiflora $\mathrm{L}$.

Silene nocturna L. subsp. boullui (Jord. ex Rouy \& Foucaud) Gamisans - Note: For the taxonomy and distribution of this subspecies, see Bacchetta et al. (2014).

Silene nocturna $\mathrm{L}$. subsp. nocturna

E Silene nodulosa Viv.

E Silene notarisii Ces.

E Silene nummica Vals.

T Silene nutans L. subsp. insubrica (Gaudin) Soldano

Silene nutans $L$. subsp. nutans

E Silene oenotriae Brullo

Silene otites (L.) Wibel

Silene paradoxa $\mathrm{L}$.

E Silene peloritana C.Brullo, Brullo, Giusso, Miniss. \& Sciandr.

Silene pendula $\mathrm{L}$.

E Silene pichiana Ferrarini \& Cecchi

Silene portensis $\mathrm{L}$.

Silene pseudoatocion Desf.

Silene pudibunda Hoffmannss. ex Rchb.

E Silene roemeri Friv. subsp. staminea (Bertol.) Nyman

E Silene rosulata Soy.-Will. \& Godr. subsp. sanctae-theresiae (Jeanm.) Jeanm.

Silene saxifraga $\mathrm{L}$.

Silene sedoides Poir. subsp. sedoides

Silene sericea All.

Silene subconica Friv.

E Silene succulenta Forssk. subsp. corsica (DC.) Nyman

Silene tenuiflora Guss.

E Silene turbinata Guss.

Silene vallesia $\mathrm{L}$.

E Silene valsecchiae Bocchieri

E Silene velutina Pourr. ex Loisel.

Silene velutinoides Pomel

Silene viridiflora $\mathrm{L}$.

E Silene vulgaris (Moench) Garcke subsp. aetnensis (Strobl) Pignatti

Silene vulgaris (Moench) Garcke subsp. commutata (Guss.) Hayek

Silene vulgaris (Moench) Garcke subsp. glareosa (Jord.) Marsden-Jones \& Turrill

Silene vulgaris (Moench) Garcke subsp. prostrata (Gaudin) Schinz \& Thell.

Silene vulgaris (Moench) Garcke subsp. tenoreana (Colla) Soldano \& F.Conti

Silene vulgaris (Moench) Garcke subsp. vulgaris

Spergula arvensis $\mathrm{L}$.

Spergula pentandra $\mathrm{L}$.

Spergularia bocconei (Scheele) Graebn.

Spergularia diandra (Guss.) Boiss.

Spergularia flaccida (Madden) I.M.Turner - Note: For the nomenclature of this species, see Turner (2017).

Spergularia heldreichii E.Simon \& P.Monnier

E Spergularia macrorhiza (Req. ex Loisel.) Heynh.

ET Spergularia madoniaca Lojac.

Spergularia marina (L.) Besser

Spergularia media (L.) C.Presl

Spergularia nicaeensis Burnat

Spergularia radicans $C$.Presl

Spergularia rubra (L.) J.Presl \& C.Presl

Spergularia segetalis (L.) G.Don

Spergularia tunetana (Maire) Jalas

Stellaria alsine Grimm

Stellaria aquatica (L.) Scop.

Stellaria graminea $\mathrm{L}$.

Stellaria holostea L. subsp. holostea

Stellaria longifolia Willd.

Stellaria media (L.) Vill. subsp. media

ET Stellaria media (L.) Vill. subsp. romana Bég.

Stellaria neglecta Weihe subsp. cupaniana (Jord. \& Fourr.) Gutermann

Stellaria neglecta Weihe subsp. neglecta

Stellaria nemorum L. subsp. montana (Pierrat) Berher

Stellaria nemorum $L$. subsp. nemorum

Stellaria pallida (Dumort.) Crép.

Stellaria palustris Ehrh. ex Hoffm.

Telephium imperati L. subsp. imperati

C Vaccaria hispanica (Mill.) Rauschert

Velezia rigida $\mathrm{L}$.

Viscaria alpina (L.) G.Don

Viscaria vulgaris Bernh. subsp. vulgaris 
Chenopodiaceae

Taxonomic references: Caroxylon Thunb., Salsola L. (incl. Kali Mill.), and Soda (Dumort.) Fourr. (Rike 1999; Akhani et al. 2007; Brullo et al. 2013, 2015a; Domina et al. 2014; Galasso and Bartolucci 2014; Sukhorukov 2014; Mosyakin 2017a; 2017c; Mosyakin et al. 2017; Wilson 2017); Chenopodium L. (lamonico 2013b); Salicornia L. (incl. Sarcocornia A.J.Scott) (Kadereit et al. 2012; Piirainen et al. 2017).

Arthrocaulon macrostachyum (Moric.) Piirainen \& G.Kadereit

Atriplex glauca L.

Atriplex halimus L.

Atriplex littoralis L.

Atriplex mollis Desf.

Atriplex oblongifolia Waldst. \& Kit.

Atriplex patula L.

Atriplex prostrata Boucher ex DC.

Atriplex rosea $\mathrm{L}$.

T Atriplex tatarica L.

Atriplex tornabenei Tineo subsp. tornabenei - Note: According to lamonico (2013c), only the autonymic subspecies occurs in Italy.

Bassia hyssopifolia (Pall.) Kuntze

Bassia laniflora (S.G.Gmel.) A.J.Scott

Bassia prostrata (L.) Beck

Beta macrocarpa Guss.

Beta vulgaris L. subsp. maritima (L.) Arcang.

Blitum bonus-henricus (L.) Rchb.

Blitum virgatum $L$. subsp. virgatum

Camphorosma monspeliaca L. subsp. monspeliaca

Caroxylon agrigentinum (Guss.) C.Brullo, Brullo, Giusso, Guarino \& lamonico

Caroxylon vermiculatum (L.) Akhani \& E.H.Roalson - Note: Salsola flavescens Cav., recorded for SAR (Botschantzev 1975; Arrigoni 2006), is provisionally included here.

Chenopodiastrum hybridum (L.) S.Fuentes, Uotila \& Borsch

Chenopodiastrum murale (L.) S.Fuentes, Uotila \& Borsch

Chenopodium album L. subsp. album - Note: Chenopodium album is a highly polymorphic species. The three subspecies, provisionally accepted here, differ on the basis of the shape and margins of leaf blades, inflorescence structure and hairness. However, further taxonomic studies including similar species such as

C. betaceum Andrz., C. probstii Aellen and C. suecicum Murr are needed.

Chenopodium album L. subsp. borbasii (Murr) Soó

Chenopodium album L. subsp. pedunculare (Bertol.) Arcang

Chenopodium betaceum Andrz. - Note: For the nomenclature of this species, see Mosyakin (2017b).

Chenopodium ficifolium Sm.

Chenopodium opulifolium Schrad. ex W.D.J.Koch \& Ziz

Chenopodium suecicum Murr

Chenopodium vulvaria $\mathrm{L}$.

C Corispermum hyssopifolium L. group - Note: The taxonomic identity of the Italian plants (possibly Corispermum bracteatum Viv., C. gallicum llijin or C. pallasii

Steven (= C. leptopterum (Asch.) Iljin) remains obscure and needs further studies (S.L. Mosyakin, in litteris). Some authors have erroneously identified the

Italian plants with the Baltic species C. intermedium Schweigg.

Dysphania botrys (L.) Mosyakin \& Clemants

E Eokochia saxicola (Guss.) Freitag \& G.Kadereit

Halimione portulacoides (L.) Aellen

Halocnemum cruciatum (Forssk.) Tod.

Halocnemum strobilaceum (Pall.) M.Bieb.

Halopeplis amplexicaulis (Vahl) Ces., Pass. \& Gibelli

Lipandra polysperma (L.) S.Fuentes, Uotila \& Borsch

Oxybasis chenopodioides (L.) S.Fuentes, Uotila \& Borsch - Note: For the taxonomy and distribution of this species, see lamonico (2014c).

Oxybasis glauca (L.) S.Fuentes, Uotila \& Borsch

Oxybasis rubra (L.) S.Fuentes, Uotila \& Borsch

Oxybasis urbica (L.) S.Fuentes, Uotila \& Borsch

Patellifolia procumbens (C.Sm.) A.J.Scott

Salicornia fruticosa $(\mathrm{L}$.) L.

Salicornia perennans Willd. subsp. perennans

Salicornia perennis Mill. subsp. alpini (Lag.) Castrov.

Salicornia perennis Mill. subsp. perennis

Salicornia procumbens Sm. subsp. procumbens

T Salicornia veneta Pignatti \& Lausi - Note: Kadereit et al. (2012) consider S. veneta as a synonym of S. procumbens. On the contrary, Iberite and lamonico (2016), based on preliminary morphological studies, treat $\mathrm{S}$. veneta as a distinct unit. Further molecular studies are ongoing by M. Iberite and collaborators.

Salsola squarrosa Steven ex Moq. subsp. controversa (Tod. ex Lojac.) Mosyakin

Salsola tragus L. - Note: Kali basalticum C.Brullo, Brullo, Gaskin, Giusso, Hrusa \& Salmeri is very similar to this species (S.L. Mosyakin and H. Freitag, in litteris), so that it is provisionally included here.

Salsola oppositifolia Desf. - Note: Salsola oppositifolia is phylogenetically close to several other Salsola species which are neatly separated (Schüssler et al. 2017) from the clade including the generitype S. kali L. However, the phylogenetic position of S. oppositifolia is not yeat clear (S.L. Mosyakin and H. Freitag, in litteris) and, for this reason, we provisionally abstain from proposing nomenclatural changes.

Soda inermis Fourr.

Spirobassia hirsuta (L.) Freitag \& G.Kadereit

E Suaeda kocheri Guss. ex C.Brullo, Brullo \& Giusso - Note: Possibly extinct.

Suaeda maritima (L.) Dumort.

E Suaeda pelagica Bartolo, Brullo \& Pavone

Suaeda spicata (Willd.) Moq.

Suaeda splendens (Pourr.) Gren. \& Godr.

Suaeda vera J.F.Gmel.

Suaeda vermiculata Forssk. ex J.F.Gmel. 
Amaranthaceae

Taxonomic references: Amaranthus L., and Polycnemum L. (lamonico 2012, 2015).

Achyranthes sicula (L.) All.

Amaranthus blitum L. subsp. blitum - Note: Amaranthus blitum is a polymorphic taxon, especially concerning characters referred to the shape of leaf blade, seed diameter and seed suface (lamonico 2015). Moreover, A. blitum belongs to a taxonomically critical group in which other species can be recognized, i.e. A. emarginatus Moq. ex Uline \& Bray (naturalized alien in Italy, see lamonico 2015), and A. bengalense Saubhik \& lamonico (from West Bengal, India) (Das and lamonico 2014).

Amaranthus graecizans L. subsp. graecizans

Amaranthus graecizans L. subsp. silvestris (Vill.) Brenan

Polycnemum arvense $\mathrm{L}$.

C Polycnemum heuffelii Láng

Polycnemum majus A.Braun

Polycnemum verrucosum Láng - Note: For the distribution of this species, see lamonico (2013d).

Aizoaceae

Aizoanthemopsis hispanicum (L.) Klak - Note: For the nomenclature of this species, see Klak et al. (2017).

Mesembryanthemum cristallinum $\mathrm{L}$.

Mesembryanthemum nodiflorum $\mathrm{L}$.

Montiaceae

Montia arvensis Wallr.

Montia fontana L.

Montia hallii (A.Gray) Greene

Montia variabilis (Walters) Landolt

Portulacaceae

Portulaca cypria Danin

Portulaca granulatostellulata (Poelln.) Ricceri \& Arrigoni

Portulaca nitida (Danin \& H.G.Baker) Ricceri \& Arrigoni

Portulaca oleracea $\mathrm{L}$.

Portulaca papillatostellulata (Danin \& H.G.Baker) Danin

Portulaca rausii Danin

E Portulaca sardoa Danin, Bagella \& Marrosu

E Portulaca sicula Danin, Domina \& Raimondo

Portulaca trituberculata Danin, Domina \& Raimondo

Portulaca zaffranii Danin

Cornaceae

Cornus mas L.

T Cornus sanguinea L. subsp. australis (C.A.Mey.) Jáv.

T Cornus sanguinea L. subsp. hungarica (Kárpáti) Soó

Cornus sanguinea $L$. subsp. sanguinea

Hydrangeaceae

Philadelphus coronarius L.

Balsaminaceae

Impatiens noli-tangere L.

Polemoniaceae

Polemonium caeruleum $\mathrm{L}$.

Primulaceae

Androsace adfinis Biroli subsp. adfinis

Androsace adfinis Biroli subsp. brigantiaca (Jord. \& Fourr.) Kress

Androsace adfinis Biroli subsp. puberula (Jord. \& Fourr.) Kress

Androsace alpina (L.) Lam.

Androsace brevis (Hegetschw.) Ces.

Androsace chamaejasme Wulfen

Androsace elongata L. subsp. breistrofferi (Charpin \& Greuter) Molero \& J.M.Monts.

Androsace hausmannii Leyb.

Androsace helvetica (L.) All.

Androsace lactea $\mathrm{L}$.

E Androsace mathildae Levier

Androsace maxima $\mathrm{L}$.

Androsace obtusifolia All.

Androsace pubescens DC.

Androsace septentrionalis $\mathrm{L}$.

Androsace vandellii (Turra) Chiov.

Androsace villosa $\mathrm{L}$. subsp. villosa

Androsace vitaliana (L.) Lapeyr. subsp. cinerea (Sünd.) Kress

Androsace vitaliana (L.) Lapeyr. subsp. lepontina (Chiarugi) Dixon, Gutermann \& Schneew.

Androsace vitaliana (L.) Lapeyr. subsp. praetutiana (Buser ex Sünd.) Kress

Androsace vitaliana (L.) Lapeyr. subsp. sesleri (Buser ex Sünd.) Kress

Androsace wulfeniana Sieber ex W.D.J.Koch

Coris monspeliensis L. subsp. annua (Halàcsy \& Bald.) Arrigoni

Coris monspeliensis $L$. subsp. monspeliensis

Cyclamen africanum Boiss. \& Reut. 
Cyclamen hederifolium Aiton subsp. confusum (Grey-Wilson) Grey-Wilson

Cyclamen hederifolium Aiton subsp. hederifolium

Cyclamen purpurascens Mill. subsp. purpurascens

Cyclamen repandum Sm. subsp. repandum

Hottonia palustris $\mathrm{L}$.

Lysimachia arvensis (L.) U.Manns \& Anderb. subsp. arvensis

T Lysimachia arvensis (L.) U.Manns \& Anderb. subsp. Iatifolia (L.) Peruzzi

T Lysimachia arvensis (L.) U.Manns \& Anderb. subsp. parviflora (Hoffmanns. \& Link) Peruzzi

Lysimachia europaea (L.) U.Manns \& Anderb.

Lysimachia foemina (Mill.) U.Manns \& Anderb.

Lysimachia linum-stellatum $\mathrm{L}$

Lysimachia minima (L.) U.Manns \& Anderb.

Lysimachia monelli (L.) U.Manns \& Anderb. subsp. linifolia (L.) Peruzzi

Lysimachia monelli (L.) U.Manns \& Anderb. subsp. monelli

Lysimachia nemorum $\mathrm{L}$.

Lysimachia nummularia $\mathrm{L}$.

Lysimachia punctata L.

Lysimachia tenella $\mathrm{L}$.

Lysimachia tyrrhenia U.Manns \& Anderb.

Lysimachia vulgaris $\mathrm{L}$.

E Primula albenensis Banfi \& Ferl.

Primula allionii Loisel.

E Primula apennina Widmer

Primula auricula L.

E Primula cottia Widmer

Primula daonensis (Leyb.) Leyb.

Primula elatior (L.) Hill

Primula farinosa $\mathrm{L}$.

E Primula glaucescens Morett

Primula glutinosa Wulfen

Primula halleri J.F.Gmel.

Primula hirsuta All.

E Primula infecta (Kress) Landolt

Primula integrifolia $\mathrm{L}$.

Primula intricata Gren. \& Godr.

Primula latifolia Lapeyr. subsp. graveolens (Hegetschw.) Rouy

Primula marginata Curtis

Primula matthioli (L.) V.A.Richt.

Primula minima $\mathrm{L}$.

E Primula palinuri Petagna

Primula pedemontana Thomas ex Gaudin

Primula polliniana Moretti

Primula recubariensis Prosser \& Scorteg.

Primula tyrolensis Schott ex Rchb.f.

Primula veris $L$. subsp. columnae (Ten.) Maire \& Petitm.

Primula veris $L$. subsp. veris

Primula vulgaris Huds. subsp. vulgaris

Primula wulfeniana Schott subsp. wulfeniana

Samolus valerandi $L$.

Soldanella alpina L. subsp. alpina

E Soldanella calabrella Kress

Soldanella minima Hoppe subsp. minima

E Soldanella minima Hoppe subsp. samnitica Cristof. \& Pignatti

Soldanella pusilla Baumg. subsp. alpicola (F.K.Mey.) Chrtek

ET Soldanella sacra A Bellino \& L Bellino

Styracaceae

Styrax officinalis L.

Ericaceae

Andromeda polifolia $\mathrm{L}$

Arbutus unedo L.

Arctostaphylos alpinus (L.) Spreng.

Arctostaphylos uva-ursi (L.) Spreng.

Calluna vulgaris (L.) Hull

Empetrum hermaphroditum Hagerup

Empetrum nigrum L.

Erica arborea $\mathrm{L}$.

Erica carnea L. subsp. carnea

Erica cinerea $\mathrm{L}$.

Erica forskalii Vitm.

E Erica multiflora L. subsp. hyblaea Domina \& Raimondo

Erica multiflora L. subsp. multiflora

Erica scoparia L. subsp. scoparia

E Erica sicula Guss. subsp. sicula

Erica terminalis Salisb.

Kalmia procumbens (L.) Gift, Kron \& Stevens ex Galasso, Banfi \& F.Conti

Moneses uniflora (L.) A.Gray 
Monotropa hypophegea Wallr.

Monotropa hypopitys L.

Orthilia secunda (L.) House

Pyrola chlorantha SW.

Pyrola media SW.

Pyrola minor L.

Pyrola rotundifolia L. subsp. rotundifolia

Rhododendron ferrugineum $\mathrm{L}$.

Rhododendron hirsutum $\mathrm{L}$.

Rhodothamnus chamaecistus (L.) Rchb.

Vaccinium microcarpum (Turcz. ex Rupr.) Schmalh.

Vaccinium myrtillus $\mathrm{L}$.

Vaccinium oxycoccos $L$

Vaccinium uliginosum L. subsp. microphyllum (Lange) Tolm.

Vaccinium uliginosum $L$. subsp. uliginosum

Vaccinium vitis-idaea $\mathrm{L}$.

Rubiaceae

Taxonomic references: Asperula L. sect. Cynanchicae (DC.) Boiss. (Del Guacchio and Caputo 2013a, 2013b; Gargiulo et al. 2015; Del Guacchio et al. 2017).

ET Asperula apuana (Fiori) Arrigoni

Asperula aristata L.f. subsp. aristata

E Asperula aristata L.f. subsp. calabra (Fiori) Del Guacchio \& P.Caputo

Asperula aristata L.f. subsp. scabra Nyman

Asperula arvensis L.

E Asperula crassifolia L. - Note: For the typification of this name, see Peruzzi et al. (2013c).

Asperula cynanchica L. subsp. cynanchica

E Asperula cynanchica L. subsp. neglecta (Guss.) Arcang.

E Asperula deficiens Viv.

E Asperula garganica Huter ex Ehrend. \& Krendl

E Asperula gussonei Boiss.

Asperula hexaphylla All.

E Asperula lactea (Huter, Porta \& Rigo ex Galasso) Brullo, Gargano, N.G.Passal. \& Peruzzi

Asperula laevigata $\mathrm{L}$.

E Asperula peloritana C.Brullo, Brullo, Giusso \& Scuderi

E Asperula pumila Moris

Asperula purpurea (L.) Ehrend.

E Asperula rupestris Tineo

E Asperula staliana Vis. subsp. diomedea Korica, Lausi \& Ehrend.

Asperula taurina L. subsp. taurina

Asperula tinctoria $\mathrm{L}$.

Crucianella angustifolia L.

Crucianella latifolia L.

Crucianella maritima L.

Crucianella rupestris Guss.

Cruciata glabra (L.) C.Bauhin ex Opiz

Cruciata laevipes Opiz

E Galium aetnicum Biv.

Galium album Mill. subsp. album

Galium album Mill. subsp. pycnotrichum (Heinr.Braun) Krendl

Galium anisophyllon Vill.

Galium aparine L.

Galium aristatum L.

Galium austriacum Jacq.

E Galium baldense Spreng.

Galium boreale L.

E Galium caprarium Natali

Galium carmineum Beauverd

Galium centroniae Cariot

Galium cinereum All.

Galium corrudifolium Vill.

Galium corsicum Spreng.

Galium debile Desv.

Galium divaricatum Lam.

E Galium glaucophyllum Em.Schmid

Galium glaucum L.

Galium intermedium Schult.

Galium laevigatum L.

E Galium litorale Guss.

Galium Iucidum All. subsp. Iucidum

ET Galium lucidum All. subsp. venustum (Jord.) Arcang.

E Galium magellense Ten.

E Galium margaritaceum A.Kern.

Galium megalospermum All.

Galium minutulum Jord.

Galium mollugo L.

E Galium montis-arerae Merxm. \& Ehrend.

Galium murale (L.) All. 
Galium noricum Ehrend.

Galium obliquum Vill.

Galium odoratum (L.) Scop.

Galium palaeoitalicum Ehrend.

Galium pallidum C.Presl

Galium palustre L. subsp. elongatum (C.Presl) Lange

Galium palustre L. subsp. palustre

Galium parisiense L.

Galium pseudohelveticum Ehrend.

Galium pumilum Murray

Galium pusillum L.

Galium rotundifolium L. subsp. rotundifolium

Galium rubrum L.

Galium saxatile L.

Galium scabrum L.

Galium schmidii Arrigon

Galium setaceum Lam.

Galium spurium $\mathrm{L}$.

Galium sylvaticum L.

Galium tendae Rchb.f.

Galium tricornutum Dandy

Galium tunetanum Lam.

Galium uliginosum $\mathrm{L}$.

ET Galium verrucosum Huds. subsp. halophilum (Ponzo) Lambinon

Galium verrucosum Huds. subsp. verrucosum

Galium verticillatum Danthoine

Galium verum $L$. subsp. verum

T Galium verum L. subsp. wirtgeni (F.W.Schultz) Oborny

Plocama calabrica (L.f.) M.Backlund \& Thulin

Rubia peregrina $\mathrm{L}$.

Sherardia arvensis L.

Theligonum cynocrambe $\mathrm{L}$.

E Valantia calva Brullo

E Valantia deltoidea Brullo

Valantia hispida $\mathrm{L}$.

Valantia muralis L.

Gentianaceae

Blackstonia acuminata (W.D.J.Koch \& Ziz) Domin subsp. acuminata

T Blackstonia acuminata (W.D.J.Koch \& Ziz) Domin subsp. aestiva (K.Malý) Zeltner

Blackstonia grandiflora (Viv.) Pau

Blackstonia imperfoliata (L.f.) Samp.

T Blackstonia perfoliata (L.) Huds. subsp. intermedia (Ten.) Zeltner

Blackstonia perfoliata (L.) Huds. subsp. perfoliata

Centaurium erythraea Rafn subsp. erythraea

T Centaurium erythraea Rafn subsp. rhodense (Boiss. \& Reut.) Melderis

T Centaurium erythraea Rafn subsp. rumelicum (Velen.) Melderis

Centaurium grandiflorum (Pers.) Ronniger subsp. grandiflorum

T Centaurium grandiflorum (Pers.) Ronniger subsp. majus (Hoffmanns. \& Link) Z.Díaz

Centaurium littorale (Turner) Gilmour subsp. littorale

Centaurium maritimum (L.) Fritsch

Centaurium pulchellum (Sw.) Druce subsp. pulchellum

T Centaurium tenuiflorum (Hoffmanns. \& Link) Fritsch subsp. acutiflorum (Schott) Zeltner

Centaurium tenuiflorum (Hoffmanns. \& Link) Fritsch subsp. tenuiflorum

Cicendia filiformis (L.) Delarbre

Exaculum pusillum (Lam.) Carue

Gentiana acaulis L.

Gentiana alpina Vill.

Gentiana asclepiadea $\mathrm{L}$.

Gentiana bavarica L.

Gentiana brachyphylla Vill.

E Gentiana brentae Prosser \& Bertolli

Gentiana burseri Lapeyr. subsp. actinocalyx Polidori

Gentiana burseri Lapeyr. subsp. villarsii (Griseb.) Rouy

Gentiana clusii E.P.Perrier \& Songeon

Gentiana cruciata L. subsp. cruciata

Gentiana dinarica Beck

Gentiana froelichii Jan ex Rchb. subsp. froelichii

E Gentiana froelichii Jan ex Rchb. subsp. zenariae F.Martini \& Poldini

Gentiana ligustica R.Vilm. \& Chopinet

Gentiana lutea L. subsp. Iutea

Gentiana lutea L. subsp. symphyandra (Murb.) Hayek

Gentiana lutea $L$. subsp. vardjanii Wraber

Gentiana nivalis L.

Gentiana orbicularis Schur

Gentiana pannonica Scop.

Gentiana pneumonanthe L. subsp. pneumonanthe 
Gentiana prostrata Haenke

Gentiana pumila Jacq. subsp. delphinensis (Beauverd) P.Fourn.

Gentiana pumila Jacq. subsp. pumila

Gentiana punctata L.

Gentiana purpurea L.

Gentiana rostanii Reut. ex Verl.

Gentiana terglouensis Hacq. subsp. schleicheri (Vacc.) Tutin

Gentiana terglouensis Hacq. subsp. terglouensis

Gentiana utriculosa L.

Gentiana verna $\mathrm{L}$. subsp. tergestina (Beck) Hayek

Gentiana verna $L$. subsp. verna

Gentianella amarella (L.) Börner

Gentianella anisodonta (Borbás) Á.Löve \& D.Löve

Gentianella austriaca (A.Kern. \& Jos.Kern.) Holub

Gentianella campestris (L.) Börner subsp. campestris

Gentianella carinthiaca (Wulfen) Galasso, Banfi \& Soldano

Gentianella columnae (Ten.) Holub

Gentianella crispata (Vis.) Holub

Gentianella engadinensis (Wettst.) Holub

Gentianella insubrica (Kunz) Holub

Gentianella lutescens (Velen.) Holub

Gentianella nana (Wulfen) N.M.Pritch.

Gentianella pilosa (Wettst.) Holub

Gentianella ramosa (Hegetschw.) Holub

Gentianella rhaetica (A.Kern. \& Jos.Kern.) Á.Löve \& D.Löve

Gentianella tenella (Rottb.) Börner

Gentianopsis ciliata (L.) Ma subsp. ciliata

Schenkia spicata (L.) G.Mans.

Swertia perennis $\mathrm{L}$.

Apocynaceae

Caralluma europaea (Guss.) N.E.Br. subsp. europaea

Cynanchum acutum L. subsp. acutum

Nerium oleander L. subsp. oleander

Periploca angustifolia Labill.

C Periploca graeca $\mathrm{L}$.

Trachomitum venetum (L.) Woodson subsp. venetum

Vinca difformis Pourr. subsp. difformis

E Vinca difformis Pourr. subsp. sardoa Stearn

Vinca major L. subsp. major

Vinca minor $\mathrm{L}$.

T Vincetoxicum hirundinaria Medik. subsp. adriaticum (Beck) Markgr.

T Vincetoxicum hirundinaria Medik. subsp. contiguum (W.D.J.Koch) Markgr.

Vincetoxicum hirundinaria Medik. subsp. hirundinaria

T Vincetoxicum hirundinaria Medik. subsp. Iaxum (Bartl.) Poldini

T Vincetoxicum hirundinaria Medik. subsp. Iuteolum (Jord. \& Fourr.) La Valva, Moraldo \& Caputo

Vincetoxicum nigrum (L.) Moench

Convolvulaceae

Taxonomic references: Convolvulus L. (incl. Calystegia R.Br.) (Stefanović et al. 2002, 2003; Carine et al. 2004; Williams et al. 2014).

Convolvulus althaeoides $\mathrm{L}$.

Convolvulus arvensis $\mathrm{L}$.

Convolvulus cantabrica L.

Convolvulus cneorum L.

Convolvulus elegantissimus Mill.

Convolvulus humilis Jacq.

Convolvulus lineatus L.

Convolvulus oleifolius Desr.

Convolvulus pentapetaloides $\mathrm{L}$.

Convolvulus sabatius Viv. subsp. sabatius

Convolvulus sepium $\mathrm{L}$.

Convolvulus siculus L. subsp. elongatus Batt.

Convolvulus siculus L. subsp. siculus

Convolvulus silvaticus Kit.

Convolvulus soldanella $\mathrm{L}$.

Convolvulus tricolor L. subsp. cupanianus (Tod.) Cavara \& Grande

Convolvulus tricolor L. subsp. meonanthus (Hoffmanns. \& Link) Arcang.

Cressa cretica L.

Cuscuta approximata Bab. subsp. approximata

Cuscuta approximata Bab. subsp. macranthera (Heldr. \& Sartoni ex Boiss.) Feinbrun \& Greuter

Cuscuta brevistyla A.Braun ex A.Rich.

Cuscuta cesattiana Bertol. - Note: This species could correspond to Cuscuta obtusiflora Kunth var. glandulosa Engelm. (Campanile 1926). Cuscuta cesattiana is usually misidentified with the alien invasive C. campestris Yunck. The specific epithet was published with double " $t$ ", possibly to avoid the pronunciation " $t z$ " in the name "Cesati", and it is not be corrected.

E Cuscuta epithymum (L.) L. subsp. corsicana (Yunck.) Lambinon

Cuscuta epithymum (L.) L. subsp. epithymum

T C Cuscuta epithymum (L.) L. subsp. kotschyi (Des Moul.) Arcang.

Cuscuta europaea L. 
Cuscuta monogyna Vahl subsp. monogyna

Cuscuta palaestina Boiss. subsp. palaestina

Cuscuta planiflora Ten.

Cuscuta scandens Brot.

Ipomoea sagittata Poir.

Ipomoea stolonifera (Cyr.) J.F.Gmel.

Solanaceae

Alkekengi officinarum Moench

Atropa bella-donna $\mathrm{L}$.

Hyoscyamus albus L.

Hyoscyamus niger $\mathrm{L}$.

C Lycium europaeum $\mathrm{L}$.

C Lycium intricatum Boiss. subsp. intricatum

Mandragora autumnalis Bertol.

Mandragora officinarum L.

Scopolia carniolica Jacq.

Solanum dulcamara $\mathrm{L}$.

Solanum nigrum $\mathrm{L}$.

Solanum villosum Mill.

Boraginaceae

Taxonomic references: Onosma echioides (L.) L. group (Peruzzi and Passalacqua 2008)

Aegonychon calabrum (Ten.) Holub.

Aegonychon purpurocaeruleum (L.) Holub

Alkanna lutea Moris

Alkanna tinctoria Tausch subsp. tinctoria

Anchusa azurea Mill.

Anchusa capellii Moris

Anchusa crispa Viv. subsp. crispa

Anchusa crispa Viv. subsp. maritima (Vals.) Selvi \& Bigazzi

Anchusa formosa Selvi, Bigazzi \& Bacch.

Anchusa litorea Moris

Anchusa montelinasana Angius, Pontec. \& Selvi

Anchusa officinalis $\mathrm{L}$.

E $\quad$ Anchusa sardoa (Illario) Selvi \& Bigazzi

Anchusa undulata L. subsp. hybrida (Ten.) Bég

Anchusella cretica (Mill.) Bigazzi, E.Nardi \& Selvi

Asperugo procumbens $\mathrm{L}$.

E Borago morisiana Bigazzi \& Ricceri

Borago officinalis $\mathrm{L}$.

E Borago pygmaea (DC.) Chater \& Greuter

Buglossoides arvensis (L.) I.M.Johnst. subsp. arvensis

Buglossoides incrassata (Guss.) I.M.Johnst. subsp. incrassata

Buglossoides incrassata (Guss.) I.M.Johnst. subsp. permixta (Jord.) L.Cecchi \& Selvi

E Buglossoides incrassata (Guss.) I.M.Johnst. subsp. splitgerberi (Guss.) E.Zippel \& Selvi

E Buglossoides minima (Moris) R.Fern.

Cerinthe alpina Kit. ex Schult. subsp. alpina

Cerinthe major L. subsp. major

Cerinthe minor L. subsp. auriculata (Ten.) Domac

Cerinthe minor L. subsp. minor

Cerinthe retorta $\mathrm{Sm}$.

E Cynoglossum apenninum $\mathrm{L}$.

E Cynoglossum barbaricinum Arrigoni \& Selvi

Cynoglossum cheirifolium $\mathrm{L}$.

Cynoglossum clandestinum Desf.

Cynoglossum columnae Ten.

Cynoglossum creticum Mill.

E Cynoglossum magellense Ten

Cynoglossum montanum L.

E Cynoglossum nebrodense Guss. subsp. Iucanum Selvi \& Sutorý

Cynoglossum nebrodense Guss. subsp. nebrodense

Cynoglossum officinale $\mathrm{L}$.

Cynoglossum pustulatum Boiss. subsp. pustulatum

Cynoglottis barrelieri (All.) Vural \& Kit Tan subsp. barrelieri

E Echium anchusoides Bacch., Brullo \& Selvi

Echium arenarium Guss.

Echium asperrimum Lam.

Echium creticum L. subsp. coyncianum (Lacaita) R.Fern.

Echium creticum L. subsp. creticum

Echium italicum L. subsp. italicum

ET Echium italicum L. subsp. siculum (Lacaita) Greuter \& Burdet

Echium parviflorum Moench

Echium plantagineum L.

Echium sabulicola Pomel subsp. sabulicola

T Echium vulgare L. subsp. pustulatum (Sm.) Em.Schmid \& Gams

Echium vulgare L. subsp. vulgare 
Eritrichium nanum (L.) Schrad. ex Gaudin Glandora rosmarinifolia (Ten.) D.C.Thomas

Hackelia deflexa (Wahlenb.) Opiz

Hormuzakia aggregata (Lehm.) Guşul.

Lappula heteracantha (Ledeb.) Gürke

Lappula squarrosa (Retz.) Dumort.

Lithospermum officinale $L$.

Lycopsis arvensis $\mathrm{L}$.

E Moltkia suffruticosa (L.) Brand subsp. bigazziana Peruzzi \& Soldano

E Moltkia suffruticosa (L.) Brand subsp. suffruticosa

Myosotis alpestris F.W.Schmidt

Myosotis arvensis (L.) Hill subsp. arvensis

Myosotis decumbens Host subsp. decumbens

ET Myosotis decumbens Host subsp. florentina Grau

Myosotis discolor Pers. subsp. discolor

E Myosotis graui Selvi

Myosotis incrassata Guss.

Myosotis laxa Lehm. subsp. cespitosa (Schultz) Hyl. ex Nordh.

Myosotis minutiflora Boiss. \& Reut. subsp. minutiflora

Myosotis nemorosa Besser

Myosotis pusilla Loisel.

Myosotis ramosissima Rochel subsp. ramosissima

Myosotis scorpioides L. subsp. scorpioides

Myosotis sicula Guss.

E Myosotis soleirolii Godr.

Myosotis speluncicola (Boiss.) Rouy

Myosotis stricta Link ex Roem. \& Schult.

T Myosotis sylvatica Hoffm. subsp. cyanea (Hayek) Vestergren

ET Myosotis sylvatica Hoffm. subsp. elongata (Strobl) Grau

T Myosotis sylvatica Hoffm. subsp. subarvensis Grau

Myosotis sylvatica Hoffm. subsp. sylvatica

E Myosotis tineoi C.Brullo \& Brullo

Neatostema apulum (L.) I.M.Johnst.

Nonea echioides (L.) Roem. \& Schult.

Nonea vesicaria (L.) Rchb.

Omphalodes verna Moench

Onosma arenaria Waldst. \& Kit. subsp. arenaria

E Onosma echioides (L.) L. subsp. angustifolia (Lehm.) Peruzzi \& N.G.Passal.

E Onosma echioides (L.) L. subsp. canescens (C.Presl) Peruzzi \& N.G.Passal.

Onosma echioides (L.) L. subsp. dalmatica (Scheele) Peruzzi \& N.G.Passal.

E Onosma echioides (L.) L. subsp. echioides

Onosma fastigiata (Braun-Blanq.) Lacaita subsp. fastigiata

Onosma helvetica (A.DC.) Boiss.

Onosma pseudoarenaria Schur subsp. fallax (Borbás) Rauschert

T Onosma pseudoarenaria Schur subsp. helvetica Rauschert

E Onosma pseudoarenaria Schur subsp. Iucana (Lacaita) Rauschert

E Onosma pseudoarenaria Schur subsp. tridentina (Wettst.) Braun-Blanq.

Onosma visianii Clementi

Pulmonaria angustifolia $\mathrm{L}$.

Pulmonaria australis (Murr) W.Sauer

Pulmonaria hirta L.

E Pulmonaria officinalis L. subsp. marzolae G.Astuti, Peruzzi, Cristof. \& P.Pupillo - Note: For the taxonony and distribution of this subspecies, see Astuti et al. (2014).

Pulmonaria officinalis L. subsp. officinalis

Pulmonaria stiriaca A.Kern

ET Pulmonaria vallarsae A.Kern. subsp. apennina (Cristof. \& Puppi) L.Cecchi \& Selvi

E Pulmonaria vallarsae A.Kern. subsp. vallarsae

Symphytum bulbosum K.F.Schimp.

E Symphytum gussonei F.W.Schultz

Symphytum officinale L.

Symphytum tanaicense Steven - Note: For the taxonomy and distribution of this species, see Cecchi and Selvi (2017).

Symphytum tuberosum L. subsp. angustifolium (A.Kern.) Nyman

Heliotropiaceae

Heliotropium europaeum $\mathrm{L}$.

E Heliotropium suaveolens M.Bieb. subsp. bocconei (Guss.) Brummitt

Heliotropium supinum $\mathrm{L}$.

Oleaceae

Chrysojasminum fruticans (L.) Banfi

Fontanesia phillyraeoides Labill.

Fraxinus angustifolia Vahl subsp. oxycarpa (M.Bieb. ex Willd.) Franco \& Rocha Afonso

Fraxinus excelsior $L$. subsp. excelsior

ET Fraxinus excelsior L. subsp. siciliensis Ilardi \& Raimondo

Fraxinus ornus $L$. subsp. ornus

Ligustrum vulgare L.

Olea europaea $\mathrm{L}$.

Phillyrea angustifolia $\mathrm{L}$. 
Phillyrea latifolia L.

Plantaginaceae

Anarrhinum bellidifolium (L.) Willd.

Anarrhinum corsicum Jord. \& Fourr.

Antirrhinum latifolium Mill.

Antirrhinum majus L. subsp. tortuosum (Bosc. ex Lam.) Rouy

Antirrhinum siculum Mill.

Callitriche brutia Petagna

Callitriche cophocarpa Sendtn.

Callitriche cribrosa Schotsman

Callitriche hermaphroditica $\mathrm{L}$.

Callitriche lenisulca Clavaud

Callitriche lusitanica Schotsman

Callitriche obtusangula Le Gall

Callitriche palustris $\mathrm{L}$.

Callitriche platycarpa Kütz.

Callitriche stagnalis Scop.

Callitriche truncata Guss. subsp. occidentalis (Rouy) Braun-Blanq.

Callitriche truncata Guss. subsp. truncata

Chaenorhinum minus (L.) Lange subsp. litorale (Willd.) Hayek

Chaenorhinum minus (L.) Lange subsp. minus

Chaenorhinum origanifolium (L.) Kostel. subsp. origanifolium

Chaenorhinum rubrifolium (Robill. \& Castagne ex DC.) Fourr. subsp. rubrifolium

Cymbalaria aequitriloba (Viv.) A.Chev. subsp. aequitriloba

E Cymbalaria glutinosa Bigazzi \& Raffaelli subsp. brevicalcarata Bigazzi \& Raffaelli

E Cymbalaria glutinosa Bigazzi \& Raffaelli subsp. glutinosa

E Cymbalaria mulleri (Moris) A.Chev.

Cymbalaria muralis G.Gaertn., B.Mey. \& Scherb. subsp. muralis

Cymbalaria muralis G.Gaertn., B.Mey. \& Scherb. subsp. visianii (Jav.) D.A.Webb

E Cymbalaria pallida (Ten.) Wettst.

E Cymbalaria pubescens (C.Presl) Cufod.

Digitalis ferruginea $\mathrm{L}$.

Digitalis grandiflora Mill.

Digitalis laevigata Waldst. \& Kit. subsp. Iaevigata

Digitalis lutea $\mathrm{L}$.

E Digitalis micrantha Roth ex Schweigg.

Digitalis purpurea $\mathrm{L}$.

Erinus alpinus $\mathrm{L}$.

Globularia alypum L.

Globularia bisnagarica $\mathrm{L}$.

Globularia cordifolia L.

Globularia incanescens Viv.

Globularia meridionalis (Podp.) O.Schwarz

ET Globularia neapolitana O.Schwarz

Globularia nudicaulis L.

Globularia repens Lam.

Gratiola officinalis L.

Hippuris vulgaris $\mathrm{L}$.

Kickxia cirrhosa (L.) Fritsch

Kickxia commutata (Bernh. ex Rchb.) Fritsch subsp. commutata

T Kickxia commutata (Bernh. ex Rchb.) Fritsch subsp. graeca (Bory \& Chaub.) R.Fern.

T Kickxia elatine (L.) Dumort. subsp. crinita (Mabille) Greuter

Kickxia elatine (L.) Dumort. subsp. elatine

T Kickxia spuria (L.) Dumort. subsp. integrifolia (Brot.) R.Fern.

Kickxia spuria (L.) Dumort. subsp. spuria

Linaria alpina (L.) Mill.

Linaria angustissima (Loisel.) Borbás

E Linaria arcusangeli Atzei \& Camarda

Linaria arvensis (L.) Desf.

E Linaria capraria Moris \& De Not.

Linaria chalepensis (L.) Mill.

Linaria cossonii Barratte

Linaria dalmatica (L.) Mill.

E Linaria flava (Poir.) Desf. subsp. sardoa (Sommier) A.Terracc.

Linaria genistifolia (L.) Mill.

Linaria micrantha (Cav.) Hoffmanns. \& Link

E Linaria multicaulis (L.) Mill. subsp. aetnensis Giardina \& Zizza

E Linaria multicaulis (L.) Mill. subsp. humilis (Guss.) De Leon., Giardina \& Zizza

E Linaria multicaulis (L.) Mill. subsp. multicaulis

Linaria pelisseriana (L.) Mill.

E Linaria pseudolaxiflora Lojac.

E Linaria purpurea (L.) Mill.

Linaria reflexa (L.) Desf. subsp. lubbockii (Batt.) Brullo

Linaria reflexa (L.) Desf. subsp. reflexa

Linaria repens (L.) Mill.

Linaria simplex (Willd.) Desf.

Linaria supina (L.) Chaz. subsp. supina 
Linaria tonzigii Lona

Linaria triphylla (L.) Mill.

Linaria vulgaris Mill. subsp. vulgaris

Littorella uniflora (L.) Asch.

Misopates calycinum Rothm.

Misopates orontium (L.) Raf.

Paederota bonarota (L.) L.

Paederota Iutea Scop.

Plantago afra L. subsp. afra

T Plantago afra L. subsp. zwierleinii (Nicotra) Brullo

Plantago albicans $\mathrm{L}$.

Plantago alpina $\mathrm{L}$.

Plantago altissima $\mathrm{L}$

Plantago amplexicaulis Cav. subsp. amplexicaulis

Plantago arenaria Waldst. \& Kit.

Plantago argentea Chaix subsp. argentea

$\mathrm{T} \quad$ Plantago argentea Chaix subsp. liburnica Ravnik

Plantago atrata Hoppe subsp. atrata

T Plantago atrata Hoppe subsp. fuscescens (Jord.) Pilg.

Plantago bellardii All. subsp. bellardii

T Plantago bellardii All. subsp. deflexa (Pilg.) Rech.f.

Plantago cornutii Gouan

Plantago coronopus $\mathrm{L}$.

Plantago crassifolia Forssk.

Plantago cupanii Guss.

Plantago humilis Guss.

Plantago lagopus L.

Plantago lanceolata $\mathrm{L}$.

Plantago macrorhiza Poir.

Plantago major L.

Plantago maritima L. subsp. maritima

Plantago maritima L. subsp. serpentina (All.) Arcang.

E Plantago media L. subsp. brutia (Ten.) Arcang. - For the taxonomy of this subspecies, see Palermo et al. (2010).

Plantago media L. subsp. media

E Plantago peloritana Lojac.

E Plantago sarda C.Presl

Plantago sempervirens Crantz

Plantago serraria L.

Plantago subulata L. - Note: A recent study (Hassemer et al. 2017), based on herbarium specimens observations only, proposed to consider P. holosteum Scop.

and $P$. grovesii Brullo as heterotypic synonyms of $P$. subulata L. Hassemer et al. (2017) do not mention other related taxa, such as $P$. sarda C.Presl and $P$. humilis

Guss. A biometric and karyological study is in progress to clarify the biogeographical and taxonomical relationship among all the taxa cited above.

Plantago weldenii Rchb.

Veronica acinifolia $\mathrm{L}$

Veronica agrestis L.

Veronica allionii Vill.

Veronica alpina $\mathrm{L}$.

Veronica anagallis-aquatica L. subsp. anagallis-aquatica

Veronica anagalloides Guss.

Veronica angustifolia (Vahl) Watzl - Note: For the taxonomy of this species, see Padilla-García et al. (2018).

Veronica aphylla L. subsp. aphylla

Veronica aphylla L. subsp. longistyla (Ball) Arcang.

Veronica arvensis $L$.

Veronica austriaca L. subsp. jacquinii (Baumg.) Watzl

Veronica barrelieri H.Schott ex Roem. \& Schult. subsp. barrelieri

Veronica barrelieri H.Schott ex Roem. \& Schult. subsp. nitens (Host) Albach

Veronica beccabunga $\mathrm{L}$.

Veronica bellidioides L.

Veronica catenata Pennell

Veronica chamaedrys L. subsp. chamaedrys

Veronica chamaedrys L. subsp. micans M.A.Fisch.

Veronica cymbalaria Bodard subsp. cymbalaria

Veronica cymbalaria Bodard subsp. panormitana (Guss.) Nyman

Veronica dillenii Crantz

Veronica fruticans Jacq.

Veronica fruticulosa $\mathrm{L}$.

Veronica hederifolia L.

Veronica kindlii Adamović - Note: For the taxonomy and distribution of this species, see Padilla-García et al. (2018). Since these authors studied only plants from Pollino and Sila Massifs in southern Italy (BAS, CAL), the records of ' $V$. austriaca' from ABR, CAM and LAZ are provisionally referred here.

Veronica maritima L.

Veronica montana $\mathrm{L}$.

Veronica officinalis L.

Veronica opaca Fr.

Veronica orsiniana Ten.

Veronica polita Fr.

Veronica praecox All.

Veronica prostrata $\mathrm{L}$.

Veronica scutellata $\mathrm{L}$.

Veronica serpyllifolia L. 
Veronica spicata L. subsp. fischeri (Trávn.) Albach

Veronica spicata L. subsp. spicata

Veronica sublobata M.A.Fisch.

Veronica trichadena Jord. \& Fourr.

Veronica triloba Opiz

Veronica triphyllos L.

Veronica urticifolia Jacq.

Veronica verna $\mathrm{L}$. subsp. brevistyla (Moris) Rouy

Veronica verna $L$. subsp. verna

Wulfenia carinthiaca Jacq.

Scrophulariaceae

Limosella aquatica L.

Scrophularia auriculata L. subsp. auriculata

Scrophularia canina $\mathrm{L}$.

Scrophularia frutescens $\mathrm{L}$.

Scrophularia hoppei W.D.J.Koch

Scrophularia lucida L.

Scrophularia morisii Vals.

Scrophularia nodosa L.

Scrophularia oblongifolia Loisel.

Scrophularia peregrina $\mathrm{L}$.

Scrophularia ramosissima Loisel.

Scrophularia scopolii Hoppe ex Pers.

E Scrophularia trifoliata L. - Note: For the typification of this name, see Peruzzi et al. (2013c).

Scrophularia umbrosa Dumort. subsp. umbrosa

Scrophularia vernalis $L$.

Verbascum alpinum Turra

Verbascum argenteum Ten

Verbascum blattaria L.

Verbascum boerhavii $L$.

Verbascum chaixii Vill. subsp. austriacum (Schott ex Roem. \& Schult.) Hayek

Verbascum chaixii Vill. subsp. chaixii

E Verbascum conocarpum Moris subsp. conocarpum

Verbascum creticum (L.) Kuntze

Verbascum densiflorum Bertol.

Verbascum Iongifolium Ten.

Verbascum lychnitis L.

Verbascum macrurum Ten.

Verbascum magellense Ten.

Verbascum mallophorum Boiss. \& Heldr.

Verbascum nigrum L.

ET Verbascum niveum Ten. subsp. garganicum (Ten.) Murb.

ET Verbascum niveum Ten. subsp. inarimense Murb.

Verbascum niveum Ten. subsp. niveum

Verbascum phlomoides $\mathrm{L}$.

Verbascum phoeniceum $\mathrm{L}$.

E Verbascum plantagineum Moris

Verbascum pulverulentum Vill.

Verbascum rotundifolium Ten.

Verbascum samniticum Ten.

E Verbascum siculum Tod. ex Lojac.

Verbascum sinuatum $\mathrm{L}$.

Verbascum thapsus L. subsp. montanum (Schrad.) Bonnier \& Layens

Verbascum thapsus L. subsp. thapsus

Lamiaceae

Taxonomic references: Stachys L. (incl. Prasium L. and Sideritis L.) (Bartolucci et al. 2014b; Galasso et al. 2016b).

Acanthoprasium frutescens (L.) Spenn.

Ajuga chamaepitys (L.) Schreb. subsp. chamaepitys

Ajuga chamaepitys (L.) Schreb. subsp. chia (Schreb.) Arcang.

Ajuga chamaepitys (L.) Schreb. subsp. suffrutescens (Willk.) Greuter \& Brudet

Ajuga genevensis $\mathrm{L}$.

Ajuga iva (L.) Schreb. subsp. iva

T Ajuga iva (L.) Schreb. subsp. pseudoiva (DC.) Briq.

Ajuga orientalis L.

Ajuga pyramidalis L.

Ajuga reptans $\mathrm{L}$.

E Ajuga tenorei C.Pres

Ballota hispanica (L.) Benth.

T Ballota nigra L. subsp. meridionalis (Bég.) Bég.

Ballota nigra L. subsp. nigra

T Ballota nigra L. subsp. uncinata (Fiori \& Bég.) Patzak

$\mathrm{T} \quad$ Ballota nigra $\mathrm{L}$. subsp. velutina (Posp.) Patzak

Betonica alopecuros L. subsp. alopecuros

E Betonica alopecuros L. subsp. divulsa (Ten.) Bartolucci \& Peruzzi

Betonica hirsuta $\mathrm{L}$.

Betonica officinalis L. - Note: Betonica serotina Host is provisionally included here. 
Chaiturus marrubiastrum (L.) Ehrh. ex Rchb.

Clinopodium acinos (L.) Kuntze subsp. acinos

Clinopodium acinos (L.) Kuntze subsp. villosum (Gaudin) Peruzzi \& F.Conti

Clinopodium alpinum (L.) Merino subsp. alpinum

Clinopodium alpinum (L.) Merino subsp. meridionale (Nyman) Govaerts

Clinopodium alpinum (L.) Merino subsp. nebrodense (A.Kern. \& Strobl) Bartolucci \& F.Conti

Clinopodium einseleanum (F.W.Schultz) Peruzzi \& F.Conti

Clinopodium grandiflorum (L.) Kuntze

Clinopodium graveolens (M.Bieb.) Kuntze

E Clinopodium minae (Lojac.) Peruzzi \& F.Conti

T Clinopodium nepeta (L.) Kuntze subsp. ascendens (Jord.) B.Bock

Clinopodium nepeta (L.) Kuntze subsp. nepeta

T Clinopodium nepeta (L.) Kuntze subsp. spruneri (Boiss.) Bartolucci \& F.Conti

T Clinopodium nepeta (L.) Kuntze subsp. sylvaticum (Bromf.) Peruzzi \& F.Conti

E Clinopodium raimondoi Spadaro, Faqi \& Mazzola

E Clinopodium sandalioticum (Bacch. \& Brullo) Bacch. \& Brullo ex Peruzzi \& F.Conti

E Clinopodium sardoum (Asch. \& Levier) Peruzzi \& F.Conti

Clinopodium serpyllifolium (M.Bieb.) Kuntze subsp. fruticosum (L.) Bräuchler

Clinopodium suaveolens (Sm.) Kuntze

Clinopodium subnudum (Waldst. \& Kit.) Kuntze

Clinopodium thymifolium (Scop.) Kuntze

Clinopodium vulgare L. subsp. arundanum (Boiss.) Nyman

Clinopodium vulgare L. subsp. vulgare

Dracocephalum austriacum $\mathrm{L}$.

Dracocephalum ruyschiana $\mathrm{L}$.

Galeopsis angustifolia Ehrh. ex Hoffm. subsp. angustifolia

Galeopsis bifida Boenn.

Galeopsis ladanum L.

Galeopsis pubescens Besser

Galeopsis reuteri Rchb.f.

Galeopsis segetum Neck.

Galeopsis speciosa Mill.

Galeopsis sulphurea Jord.

Galeopsis tetrahit L.

Glechoma hederacea L.

Glechoma hirsuta Waldst. \& Kit.

E Glechoma sardoa (Bég.) Bég.

Horminum pyrenaicum $\mathrm{L}$.

$T \quad$ Hyssopus officinalis $L$. subsp. aristatus (Godr.) Nyman

Hyssopus officinalis L. subsp. officinalis

Lamium album L. subsp. album

Lamium amplexicaule $\mathrm{L}$.

Lamium bifidum Cirillo subsp. balcanicum Velen.

Lamium bifidum Cirillo subsp. bifidum

Lamium flexuosum Ten.

Lamium galeobdolon (L.) L. subsp. flavidum (F.Herm.) A.Löve \& D.Löve

Lamium galeobdolon (L.) L. subsp. galeobdolon

Lamium galeobdolon (L.) L. subsp. montanum (Pers.) Hayek

E Lamium garganicum L. subsp. corsicum (Godr. \& Gren.) Arcang.

Lamium garganicum L. subsp. garganicum

Lamium garganicum L. subsp. laevigatum Arcang.

Lamium garganicum L. subsp. striatum (Sm.) Hayek

Lamium hybridum Vill.

Lamium maculatum $\mathrm{L}$.

Lamium orvala $\mathrm{L}$.

Lamium purpureum $\mathrm{L}$.

Lavandula angustifolia Mill.

E Lavandula austroapennina N.G.Passal., Tundis \& Upson

Lavandula latifolia Medik.

Lavandula multifida $\mathrm{L}$.

Lavandula stoechas $L$. subsp. stoechas

Lycopus europaeus $L$.

Lycopus exaltatus L.f.

Marrubium alysson $\mathrm{L}$.

Marrubium incanum Desr.

Marrubium vulgare $\mathrm{L}$.

Melissa officinalis L. subsp. altissima (Sm.) Arcang.

C Melissa officinalis L. subsp. officinalis

Melittis melissophyllum L. subsp. albida (Guss.) P.W.Ball

Melittis melissophyllum L. subsp. melissophyllum

Mentha aquatica L. subsp. aquatica

Mentha arvensis L.

Mentha cervina $\mathrm{L}$.

Mentha longifolia (L.) L.

Mentha microphylla K.Koch

Mentha pulegium L. subsp. pulegium

E Mentha requienii Benth. subsp. bistaminata Mannocci \& Falconcini

E Mentha requienii Benth. subsp. requienii 
Mentha spicata $\mathrm{L}$.

Mentha suaveolens Ehrh. subsp. insularis (Req.) Greuter

Mentha suaveolens Ehrh. subsp. suaveolens

Micromeria cordata (Moris ex Bertol.) Moris

Micromeria filiformis (Aiton) Benth. subsp. filiformis

ET Micromeria graeca (L.) Benth. ex Rchb. subsp. consentina (Ten.) Guinea

T Micromeria graeca (L.) Benth. ex Rchb. subsp. fruticulosa (Bertol.) Guinea

ET Micromeria graeca (L.) Benth. ex Rchb. subsp. garganica (Briq.) Guinea

Micromeria graeca (L.) Benth. ex Rchb. subsp. graeca

ET Micromeria graeca (L.) Benth. ex Rchb. subsp. Iongiflora (C.Presl) Nyman

ET Micromeria graeca (L.) Benth. ex Rchb. subsp. tenuifolia (Ten.) Nyman

Micromeria juliana (L.) Benth. ex Rchb.

Micromeria marginata (Sm.) Chater

Micromeria microphylla (d'Urv.) Benth.

Micromeria nervosa (Desf.) Benth.

Moluccella spinosa $\mathrm{L}$.

Nepeta apuleii Ucria

Nepeta cataria L.

E Nepeta foliosa Moris

Nepeta italica L.

Nepeta nepetella L. subsp. nepetella

Nepeta nuda L. subsp. nuda

Nepeta tuberosa L. subsp. tuberosa

Origanum onites $L$.

T Origanum vulgare L. subsp. prismaticum Gaudin

T Origanum vulgare L. subsp. viridulum (Martrin-Donos) Nyman

Origanum vulgare L. subsp. vulgare

Phlomis fruticosa $\mathrm{L}$.

Phlomis herba-venti L. subsp. herba-venti

DD Phlomis lanata Willd.

E Phlomis tenorei Soldano

Prunella grandiflora (L.) Scholler

Prunella hyssopifolia L.

Prunella laciniata (L.) L.

Prunella vulgaris $L$. subsp. vulgaris

Salvia aethiopis L.

Salvia argentea $\mathrm{L}$.

E Salvia ceratophylloides Ard.

Salvia clandestina $\mathrm{L}$.

E Salvia desoleana Atzei \& V.Picci

ET Salvia fruticosa Mill. subsp. thomasii (Lacaita) Brullo, Guglielmo, Pavone \& Terrasi

Salvia glutinosa $\mathrm{L}$.

E Salvia haematodes $L$.

Salvia nemorosa L. subsp. nemorosa

T Salvia officinalis L. subsp. gallica (W.Lippert) Reales, D.Rivera \& Obón

Salvia officinalis $L$. subsp. officinalis

Salvia pratensis L. subsp. pratensis

E Salvia pratensis L. subsp. saccardiana (Pamp.) Poldini

Salvia rosmarinus Schleid.

Salvia sclarea $\mathrm{L}$.

Salvia verbenaca $L$.

Salvia verticillata $L$. subsp. verticillata

Salvia virgata Jacq

Salvia viridis $\mathrm{L}$

Satureja cuneifolia Ten.

Satureja montana L. subsp. montana

Satureja montana L. subsp. variegata (Host) P.W.Ball

Satureja subspicata Bartl. ex Vis. subsp. liburnica Šilic

Satureja thymbra L.

Scutellaria alpina L. subsp. alpina

Scutellaria altissima $\mathrm{L}$.

Scutellaria columnae All. subsp. columnae

ET Scutellaria columnae All. subsp. gussonei (Ten.) Arcang.

Scutellaria galericulata L.

Scutellaria hastifolia L.

Scutellaria minor Huds.

E Scutellaria rubicunda Hornem.

Stachys alpina $L$. subsp. alpina

Stachys annua (L.) L. subsp. annua

Stachys arenaria Vahl subsp. arenaria

Stachys arvensis (L.) L.

Stachys brachyclada Noë ex Coss.

E Stachys corsica Pers.

Stachys cretica L.

ET Stachys germanica L. subsp. dasyanthes (Raf.) Arcang.

Stachys germanica L. subsp. germanica

T Stachys germanica L. subsp. salviifolia (Ten.) Gams

E Stachys glutinosa L. 
Stachys guillonii (Timb.-Lagr.) Soldano, Peruzzi \& Bartolucci subsp. hyssopifolia (L.) Soldano, Peruzzi \& Bartolucci

Stachys heraclea All.

E Stachys italica Mill.

Stachys major (L.) Bartolucci \& Peruzzi

Stachys maritima Gouan

Stachys marrubiifolia Viv.

Stachys montana (L.) Peruzzi \& Bartolucci subsp. montana

Stachys ocymastrum (L.) Briq.

Stachys palustris $\mathrm{L}$.

T Stachys recta L. subsp. grandiflora (Caruel) Arcang.

Stachys recta L. subsp. recta

T Stachys recta L. subsp. subcrenata (Vis.) Briq.

ET Stachys recta $\mathrm{L}$. subsp. tenoreana Bornm.

Stachys romana (L.) E.H.L.Krause

Stachys rossii (Peris, Stübing, Jury \& Rejdali) Bartolucci, Peruzzi \& Soldano

E Stachys salisii Jord. \& Fourr.

Stachys sylvatica $\mathrm{L}$.

Stachys thirkei K.Koch

Stachys tymphaea Hausskn.

Teucrium botrys $\mathrm{L}$.

Teucrium campanulatum $\mathrm{L}$.

Teucrium capitatum L. subsp. capitatum

Teucrium chamaedrys $L$. subsp. chamaedrys

Teucrium chamaedrys $L$. subsp. pectinatum Rech.f.

Teucrium creticum $L$.

Teucrium flavum $L$. subsp. flavum

Teucrium flavum L. subsp. glaucum (Jord. \& Fourr.) Ronniger

Teucrium fruticans $L$. subsp. fruticans

Teucrium lucidum $\mathrm{L}$.

Teucrium luteum (Mill.) Degen

Teucrium marum $\mathrm{L}$.

Teucrium massiliense $\mathrm{L}$.

Teucrium montanum L.

Teucrium polium L. subsp. polium

Teucrium polium L. subsp. purpurascens (Benth.) S.Puech

Teucrium scordium L. subsp. scordioides (Schreb.) Arcang.

Teucrium scordium L. subsp. scordium

Teucrium scorodonia $\mathrm{L}$.

E Teucrium siculum (Raf.) Guss. subsp. euganeum (Vis.) Tornad.

Teucrium siculum (Raf.) Guss. subsp. siculum

Teucrium spinosum $\mathrm{L}$.

Teucrium subspinosum Pourr. ex Willd.

Thymbra capitata (L.) Cav.

Thymus carstiensis (Velen.) Ronniger - Note: This species was considered as a hybrid between Th. Iongicaulis C.Presl and Th. pulegioides L., but it has been recently revaluated as an independent species (Bartolucci et al. 2017c).

Thymus herba-barona Loisel. - Note: For the taxonomy of this species, see Molins et al. (2011).

Thymus illyricus Ronniger

Thymus longicaulis C.Presl subsp. longicaulis - Note: All the Alpine records of this species have to be referred to other taxa (Bartolucci and Domina 2014).

Thymus moesiacus Velen. - Note: Several records of Th. oenipontanus Heinr.Braun ex Borbás from central and southern Italy should be referred to this species. Thymus moesiacus [ $=$ Th. chaubardii (Boiss. \& Heldr. ex Rchb.f.) Čelak. var. subisophyllus Borbás $\equiv$ Th. Iongicaulis C.Presl var. subisophyllus (Borbás) Jalas] is closely related to Th. glabrescens Willd. subsp. urumovii (Velen.) Jalas. [= Th. callieri Borbás ex Velen. var. microcalyx Degen \& Urum.], and further studies are needed to establish the systematic relationships among these taxa.

Thymus oenipontanus Heinr.Braun ex Borbás - Note: The distribution of this species needs further investigations. For its nomenclature, see Bartolucci and Scharfetter (2015).

Thymus paronychioides Čelak. - Note: For the nomenclature, taxonomy and distribution of this species, belonging to Th. sect. Hyphodromi (A. Kern.) Halácsy, see Bartolucci and Peruzzi (2014) and Bartolucci and Domina (2015).

E Thymus picentinus (Lacaita) Bartolucci - Note: The records of Th. thracicus Velen. from southern Italy have to be referred to this species (Bartolucci et al. 2013; Bartolucci and Mráz 2016).

E Thymus praecox Opiz subsp. parvulus (Lojac.) Bartolucci, Peruzzi \& N.G.Passal.

Thymus praecox Opiz subsp. polytrichus (A.Kern ex Borbás) Jalas

Thymus praecox Opiz subsp. praecox

T Thymus pseudochamaedrys (Heinr.Braun) Ronniger - Note: The taxonomic identity of this species, closely related to Th. oenipontanus Heinr.Braun ex Borbás, is still unclear. In addition, both these names are yet to be typified.

Thymus pulegioides L. - Note: It is a highly polymorphic species, which is generally divided into different subspecies [i.e. Th. pulegioides subsp. chamaedrys (Fr.) Guşul., subsp. effusus (Host) Ronniger, subsp. montanus (Benth.) Ronniger and subsp. pannonicus (All.) Kerguélen] on the basis of calyx length, leaf length and width, inflorescence length and leaf hairiness. Considering the morphological variability observed in the putative diagnostic characters, the constant presence of intermediate forms, the absence of any ecological or geographical discontinuity, it is impossible to justify any subdivision of Th. pulegioides in subspecies. Waiting for further studies, we can recognize just two varieties: the glabrous plants pertain to var. pulegioides (Th. glaber Mill., Th. ovatus Mill., Th. chamaedrys Fr., Th. pulegioides subsp. montanus (Benth.) Ronniger, incl. Th. istriacus Heinr. Braun, Th. alpestris auct. Fl. Ital.) and hairy plants pertain to var. vestitus (Lange) Jalas (Th. lanuginosus Mill., Th. pannonicus All., Th. froelichianus Opiz, Th. carniolicus Borbás ex Déségl., Th. valderius Ronniger) (Bartolucci et al. 2013).

E Thymus richardii Pers. subsp. nitidus (Guss.) Jalas

E Thymus spinulosus Ten.

Thymus striatus Vahl subsp. acicularis (Waldst. \& Kit.) Ronniger

Thymus striatus Vahl subsp. striatus

Thymus vallicola (Heinr.Braun) Ronniger

Thymus vulgaris $L$. subsp. vulgaris 
Thymus zygiformis Heinr.Braun - Note: The records of Th. thracicus Velen. from central Italy have to be referred to this species (Bartolucci and Walter 2015). Only Th. zygiformis var. magellensis (Ronniger) Bartolucci \& J.Walter occurs in Italy.

Vitex agnus-castus L.

Orobanchaceae

Taxonomic references: Bartsia L., Bellardia All., and Parentucellia Viv. (Uribe-Convers and Tank 2016); Odontites Ludw. (Pinto-Carrasco et al. 2017); Phelipanche Pomel (Domina 2017).

Bartsia alpina L.

Bellardia trixago (L.) All.

Bellardia viscosa (L.) Fisch. \& C.A.Mey.

Euphrasia alpina Lam. subsp. alpina

Euphrasia alpina Lam. subsp. christii (Favrat) Hayek

Euphrasia cisalpina Pugsley

Euphrasia cuspidata Host

Euphrasia hirtella Jord. ex Reut.

Euphrasia illyrica Wettst.

Euphrasia italica Wettst.

Euphrasia liburnica Wettst.

Euphrasia marchesettii Wettst. ex Marches.

Euphrasia micrantha Rchb.

Euphrasia minima Jacq. ex DC.

Euphrasia nana (Rouy) Prain

Euphrasia officinalis L. subsp. kerneri (Wettst.) Eb.Fisch.

Euphrasia officinalis L. subsp. picta (Wimm.) Oborny

Euphrasia officinalis L. subsp. rostkoviana (Hayne) Towns.

Euphrasia portae Wettst.

Euphrasia salisburgensis Funck ex Hoppe

Euphrasia stricta D.Wolff ex J.F.Lehm.

Euphrasia tricuspidata $\mathrm{L}$.

Lathraea clandestina $\mathrm{L}$.

Lathraea squamaria $L$.

Melampyrum arvense $\mathrm{L}$. subsp. arvense

Melampyrum barbatum Waldst. \& Kit. subsp. carstiense Ronniger

Melampyrum catalaunicum Freyn

Melampyrum cristatum L. subsp. cristatum

Melampyrum fimbriatum Vandas

E Melampyrum italicum (Beauverd) Soó

Melampyrum nemorosum $\mathrm{L}$.

T Melampyrum pratense L. subsp. commutatum (Tausch ex A.Kern.) C.E.Britton

Melampyrum pratense L. subsp. pratense

Melampyrum sylvaticum $L$. subsp. sylvaticum

Melampyrum variegatum (Porta \& Rigo) Huter

Melampyrum velebiticum Borbás

ET Odontites bocconei (Guss.) Walp. subsp. angustifolius (Lojac.) Giardina \& Raimondo

E Odontites bocconei (Guss.) Walp. subsp. bocconei

E Odontites corsicus (Loisel.) G.Don

Odontites luteus (L.) Clairv. subsp. Ianceolatus (Gaudin) P.Fourn.

Odontites luteus (L.) Clairv. Subsp. Iuteus

E Odontites rigidifolius (Biv.) Benth.

E Odontites sillettii Brullo, V.Tomas. \& Wagens. - Note: For the taxonomy and distribution of this species, see Brullo et al. (2015b).

Odontites vernus (Bellardi) Dumort. subsp. serotinus (Dumort.) Corb.

Odontites vernus (Bellardi) Dumort. subsp. siculus (Guss.) P.D.Sell

Odontites vernus (Bellardi) Dumort. subsp. vernus

Odontites viscosus (L.) Clairv. subsp. viscosus

Orobanche alba Stephan ex Willd.

Orobanche alsatica Kirschl.

Orobanche amethystea Thuill.

E Orobanche apuana Domina \& Soldano

Orobanche artemisiae-campestris Gaudin

$E$

Orobanche australis Moris ex Bertol.

Orobanche balsensis (J.A.Guim.) Carlón, M.Laínz, Moreno Mor. \& Ó.Sánchez

Orobanche canescens C.Presl

Orobanche caryophyllacea Sm.

Orobanche cernua Loefl.

E Orobanche chironii Lojac.

Orobanche crenata Forssk.

Orobanche crinita Viv.

Orobanche cumana Wallr.

E Orobanche denudata Moris

E Orobanche ebuli Huter \& Rigo

Orobanche elatior Sutton

Orobanche flava Mart. ex F.W.Schultz

Orobanche foetida Poir.

Orobanche gracilis $\mathrm{Sm}$.

Orobanche hederae Vaucher ex Duby

Orobanche kochii F.W.Schultz

Orobanche laserpitii-sileris Reut. ex Jord.

Orobanche litorea Guss. 
Orobanche lucorum A.Braun ex F.W.Schultz

Orobanche lutea Baumg.

Orobanche lycoctoni Rhiner

Orobanche minor $\mathrm{Sm}$.

Orobanche pancicii Beck

Orobanche pubescens d'Urv.

Orobanche rapum-genistae Thuill.

Orobanche reticulata Wallr. subsp. reticulata

E Orobanche rigens Loisel.

Orobanche salviae F.W.Schultz

Orobanche sanguinea C.Presl

Orobanche teucrii Holandre

Orobanche thapsoides Lojac

Orobanche variegata Wall.r.

Parentucellia latifolia (L.) Caruel

Pedicularis acaulis Scop.

Pedicularis ascendens Schleich. ex Gaudin

Pedicularis aspleniifolia Flörke ex Willd.

Pedicularis cenisia Gaudin

Pedicularis comosa L. subsp. comosa

E Pedicularis elegans Ten.

Pedicularis elongata A.Kern. subsp. elongata

Pedicularis elongata A.Kern. subsp. julica (E.Mayer) Hartl

Pedicularis foliosa $\mathrm{L}$.

Pedicularis friderici-augusti Tomm.

Pedicularis gyroflexa Vill.

Pedicularis hacquetii Graf

Pedicularis hoermanniana K.Malý

Pedicularis kerneri Dalla Torre

Pedicularis oederi Vahl

Pedicularis palustris $L$. subsp. palustris

Pedicularis petiolaris Ten.

Pedicularis recutita $\mathrm{L}$.

Pedicularis rosea Wulfen subsp. allionii (Rchb.f.) Arcang.

Pedicularis rosea Wulfen subsp. rosea

Pedicularis rostratocapitata Crantz

Pedicularis rostratospicata Crantz subsp. helvetica (Steininger) O.Schwarz

Pedicularis rostratospicata Crantz subsp. rostratospicata

Pedicularis sylvatica L. subsp. sylvatica

Pedicularis tuberosa $\mathrm{L}$.

Pedicularis verticillata $L$. subsp. verticillata

Phelipanche arenaria (Borkh.) Pomel

Phelipanche lavandulacea (Rchb.) Pomel subsp. lavandulacea

Phelipanche mutelii (F.W.Schultz) Reut.

Phelipanche nana (Reut.) Soják

Phelipanche oxyloba (Reut.) Soják

Phelipanche purpurea (Jacq.) Soják

Phelipanche ramosa (L.) Pomel

Phelipanche rosmarina (Welw. ex Beck) Banfi, Galasso \& Soldano

Phelipanche schultzii (Mutel) Pomel

Rhinanthus alectorolophus (Scop.) Pollich subsp. alectorolophus

Rhinanthus angustifolius C.C.Gmel. subsp. angustifolius

Rhinanthus antiquus (Sterneck) Schinz \& Thell.

E Rhinanthus apuanus Soldano

Rhinanthus burnatii (Chabert) Soó

Rhinanthus facchinii Chabert

Rhinanthus freynii (Sterneck) Fiori

Rhinanthus glacialis Personnat subsp. glacialis

E Rhinanthus helenae Chabert

Rhinanthus minor L.

Rhinanthus ovifugus Chabert

Rhinanthus pampaninii Chabert subsp. pampaninii

Rhinanthus pampaninii Chabert subsp. simplex Zirnich \& Cohrs

Rhinanthus pumilus (Sterneck) Pau

Rhinanthus rumelicus Velen.

Rhinanthus songeonii Chabert

E Rhinanthus wettsteinii (Sterneck) Soó

Rhynchocorys elephas (L.) Griseb.

Tozzia alpina L. subsp. alpina

Verbenaceae

Verbena officinalis $\mathrm{L}$.

Verbena supina $\mathrm{L}$.

Linderniaceae

Lindernia procumbens (Krocker) Philcox 
Acanthaceae

Acanthus mollis L. subsp. mollis

Acanthus spinosus L.

Lentibulariaceae

Taxonomic references: Utricularia L. (Astuti and Peruzzi, 2018).

Pinguicula alpina $\mathrm{L}$.

E Pinguicula apuana Casper \& Ansaldi

E Pinguicula christinae Peruzzi \& Gestri

E Pinguicula fiorii Tammaro \& Pace

C Pinguicula grandiflora Lam. subsp. grandiflora

Pinguicula hirtiflora Ten.

E Pinguicula lattanziae Peruzzi \& Gestri

ET Pinguicula lavalvae Innangi \& Izzo - Note: According to De Castro et al. (2016), this species falls within the infraspecific variability of $P$. hirtiflora Pinguicula leptoceras Rchb. Note: The nomenclature and taxonomy of the name P. arvetii Genty require further studies (De Castro et al. 2016).

E Pinguicula mariae Casper

E Pinguicula poldinii J.Steiger \& Casper

Pinguicula reichenbachiana Schindl.

E Pinguicula sehuensis Bacch., Carras \& Peruzzi

Pinguicula vallis-regiae F.Conti \& Peruzzi

Pinguicula vulgaris L. subsp. anzalonei Peruzzi \& F.Conti

Pinguicula vulgaris L. subsp. ernica Peruzzi \& F.Conti

Pinguicula vulgaris L. subsp. vestina F.Conti \& Peruzzi

Pinguicula vulgaris L. subsp. vulgaris

Utricularia australis R.Br.

T Utricularia bremii Heer ex Koell.

Utricularia minor $\mathrm{L}$.

Utricularia stygia G.Thor

Utricularia vulgaris $\mathrm{L}$.

Aquifoliaceae

Ilex aquifolium L.

Campanulaceae

Adenophora liliifolia (L.) Ledeb. ex A.DC.

Asyneuma limonifolium (L.) Janch. subsp. limonifolium

Asyneuma trichocalycinum (Ten.) K.Maly

Campanula alpestris All.

Campanula barbata $\mathrm{L}$.

Campanula bertolae Colla

Campanula bononiensis $\mathrm{L}$

Campanula carnica Schiede ex Mert. \& W.D.J.Koch subsp. carnica

E Campanula carnica Schiede ex Mert. \& W.D.J.Koch subsp. puberula Podlech

Campanula cenisia $\mathrm{L}$.

Campanula cervicaria $\mathrm{L}$.

Campanula cespitosa Scop.

Campanula cochleariifolia Lam.

Campanula dichotoma $\mathrm{L}$.

E Campanula elatines L.

E Campanula elatinoides Moretti

Campanula erinus $\mathrm{L}$.

Campanula excisa Schleich. ex Murith

Campanula foliosa Ten.

E Campanula forsythii (Arcang.) Bég.

E Campanula fragilis Cirillo subsp. cavolinii (Ten.) Damboldt

E Campanula fragilis Cirillo subsp. fragilis

Campanula fritschii Witasek

E Campanula garganica Ten. subsp. garganica

Campanula glomerata $\mathrm{L}$.

E Campanula isophylla Moretti

Campanula latifolia L.

Campanula lingulata Waldst. \& Kit.

Campanula macrorhiza J.Gay ex A.DC

E Campanula martinii F.Fen., Pistarino, Peruzzi \& Cellin. - Note: For the taxonomy and distribution of this species, see Fenaroli et al. (2013).

Campanula medium $\mathrm{L}$.

E Campanula micrantha Bertol.

E Campanula morettiana Rchb.

Campanula patula L. subsp. jahorinae (K.Malý) Greuter \& Burdet

Campanula patula L. subsp. patula

Campanula persicifolia L. subsp. persicifolia

Campanula persicifolia L. subsp. sessiliflora (K.Koch) Velen.

E Campanula petraea $\mathrm{L}$.

Campanula pyramidalis L.

E Campanula raineri Perp.

Campanula ramosissima Sm.

Campanula rapunculoides $\mathrm{L}$. subsp. rapunculoides

Campanula rapunculus $\mathrm{L}$. 
Campanula reatina Lucchese

Campanula rhomboidalis $\mathrm{L}$.

E Campanula rotundifolia L. S

E Campanula scheuchzeri Vill. subsp. pollinensis (Podlech) Bernardo, Gargano \& Peruzzi - Note: Giardina et al. (2007) consider C. scheuchzeri subsp. marcenoi (Brullo) Raimondo as a further subspecies, endemic to Sicily. However, these authors do not provide further experimental data to support their view, with respect to those presented by Bernardo et al. (2004). The latter authors, after a morphometric study, included C. marcenoi (Brullo 1993) within the variability of $C$. scheuchzeri subsp. pollinensis, and their proposal is supported here.

E Campanula scheuchzeri Vill. subsp. pseudostenocodon (Lacaita) Bernardo, Gargano \& Peruzzi

Campanula scheuchzeri Vill. subsp. scheuchzeri

Campanula sibirica L. subsp. divergentiformis (Jáv.) Domin

Campanula sibirica L. subsp. sibirica

Campanula spicata $\mathrm{L}$.

Campanula stenocodon Boiss. \& Reut.

E Campanula tanfanii Podlech

Campanula thyrsoides L. subsp. carniolica (Sünd.) Podlech

Campanula thyrsoides L. subsp. thyrsoides

Campanula trachelium L. subsp. trachelium

Campanula versicolor Andrews

Campanula witasekiana Vierh.

Campanula zoysii Wulfen

Edraianthus graminifolius (L.) A.DC. subsp. graminifolius

ET Edraianthus graminifolius (L.) A.DC. subsp. siculus (Strobl) Greuter \& Burdet

Jasione laevis Lam. subsp. Iaevis

Jasione maritima (Duby) Merino

Jasione montana L.

Jasione orbiculata Griseb. ex Velen.

E Jasione sphaerocephala Brullo, Marcenò \& Pavone

$\mathrm{T} \quad$ Legousia falcata (Ten.) Janch. subsp. castellana (Lange) Jauzein

Legousia falcata (Ten.) Janch. subsp. falcata

Legousia hybrida (L.) Delarbre

Legousia speculum-veneris (L.) Chaix

Physoplexis comosa (L.) Schur

Phyteuma betonicifolium Vill.

Phyteuma charmelii Vill.

Phyteuma cordatum Balb.

Phyteuma globulariifolium Sternb. \& Hoppe subsp. globulariifolium

Phyteuma globulariifolium Sternb. \& Hoppe subsp. pedemontanum (Rich.Schulz) Becherer

Phyteuma hedraianthifolium Rich.Schulz

Phyteuma hemisphaericum $\mathrm{L}$.

Phyteuma humile Schleich. ex Gaudin

Phyteuma italicum Arv.-Touv.

Phyteuma michelii All.

Phyteuma orbiculare L.

Phyteuma ovatum Honck. subsp. ovatum

E Phyteuma ovatum Honck. subsp. pseudospicatum Pignatti

Phyteuma scheuchzeri All. subsp. columnae (Gaudin) Bech.

Phyteuma scheuchzeri All. subsp. scheuchzeri

Phyteuma sieberi Spreng.

Phyteuma spicatum L. subsp. occidentale Rich.Schulz

Phyteuma spicatum L. subsp. spicatum

Phyteuma villarsii Rich.Schulz

Phyteuma zahlbruckneri Vest

Solenopsis bivonae (Tineo) M.B.Crespo, Serra \& Juan

E Solenopsis corsica (Meikle) M.B.Crespo, Serra \& Juan

Solenopsis Iaurentia (L.) C.Presl

ET Solenopsis mothiana C.Brullo, Brullo \& Giusso

E Trachelium caeruleum L. subsp. Ianceolatum (Guss.) Arcang.

Wahlenbergia lobelioides (L.f.) Link subsp. nutabunda (Guss.) Murb.

Menyanthaceae

Menyanthes trifoliata $L$.

Nymphoides peltata (S.G.Gmel.) Kuntze

Asteraceae

Taxonomic references: Centaurea L. (Domina et al. 2016; 2017a); Centaurea rupestris L. group (Conti et al. 2011); Jacobaea Mill. (Nordenstam and Greuter 2006; Peruzzi et al. 2006; 2007; Passalacqua et al. 2008; Galasso and Bartolucci 2015); Leontopodium R.Br. ex Cass. (Blöch et al. 2010); Omalotheca Cass. (Nie et al. 2016; Bartolucci et al. 2017b); Senecio L. sect. Crociseris (Reich.) Boiss. (Calvo et al. 2015); Taraxacum F.H.Wigg. (Kirschner and Štepánek 1998; Arrigoni et al. 2006; Arrigoni 2007, 2012; Kirschner et al. 2007 onwards; Uhlemann 2007, 2017; Aquaro et al. 2008; 2009; Peruzzi et al. 2009; Carlesi and Peruzzi 2012; Štepánek and Kirschner 2012).

Achillea ageratum L.

Achillea atrata $\mathrm{L}$.

E Achillea barrelieri (Ten.) Sch.Bip. subsp. barrelieri

E Achillea barrelieri (Ten.) Sch.Bip. subsp. elegans (Fiori) Bazzich.

Achillea barrelieri (Ten.) Sch.Bip. subsp. mucronulata (Bertol.) Heimerl

Achillea barrelieri (Ten.) Sch.Bip. subsp. oxyloba (DC.) F.Conti \& Soldano

Achillea clavennae $\mathrm{L}$. 
Achillea collina (Becker ex Wirtg.) Heimerl

Achillea distans Waldst. \& Kit. ex Willd. subsp. distans

Achillea distans Waldst. \& Kit. ex Willd. subsp. stricta (Gremli) Janch.

Achillea distans Waldst. \& Kit. ex Willd. subsp. tanacetifolia (Fiori) Janch.

Achillea erba-rotta All. subsp. erba-rotta

Achillea erba-rotta All. subsp. moschata (Wulfen) I.Richardson

Achillea ligustica All.

Achillea macrophylla L.

Achillea maritima (L.) Ehrend. \& Y.P.Guo subsp. maritima

Achillea millefolium $\mathrm{L}$. subsp. millefolium

Achillea millefolium L. subsp. sudetica (Opiz) Oborny

Achillea nana $\mathrm{L}$.

Achillea nobilis L. subsp. neilreichii (A.Kern.) Velen.

Achillea nobilis $L$. subsp. nobilis

Achillea pannonica Scheele

Achillea pratensis Saukel \& R.Länger

Achillea ptarmica $\mathrm{L}$.

Achillea roseoalba Ehrend.

ET Achillea rupestris Huter, Porta \& Rigo subsp. calcarea (Huter, Porta \& Rigo) Greuter

E Achillea rupestris Huter, Porta \& Rigo subsp. rupestris

Achillea setacea Waldst. \& Kit.

E Achillea tenorei Grande

Achillea tomentosa $\mathrm{L}$.

Achillea virescens (Fenzl) Heimerl

Adenostyles alliariae (Gouan) A.Kern.

Adenostyles alpina (L.) Bluff \& Fingerh. subsp. alpina

E Adenostyles alpina (L.) Bluff \& Fingerh. subsp. macrocephala (Huter, Porta \& Rigo) Dillenb. \& Kadereit

E Adenostyles alpina (L.) Bluff \& Fingerh. subsp. nebrodensis (Wagenitz \& I.Müll.) Greuter

E Adenostyles australis (Ten.) lamonico \& Pignatti

Adenostyles leucophylla (Willd.) Rchb.

Ambrosia maritima L. - Note: The occurrence of this taxon in Italy is confirmed only in SAR (Orsenigo et al. 2017).

Anacyclus clavatus (Desf.) Pers.

Anacyclus radiatus Loisel. subsp. radiatus

Andryala cossyrensis Guss.

Andryala dentata $\mathrm{Sm}$.

Andryala integrifolia $\mathrm{L}$.

Andryala rothia Pers.

E Andryala tenuifolia (Tineo) DC

Antennaria carpatica (Wahlenb.) Bluff \& Fingerh. subsp. helvetica (Chrtek \& Pouzar) Chrtek \& Pouzar

Antennaria dioica (L.) Gaertn.

Anthemis aeolica Lojac.

Anthemis aetnensis Spreng.

Anthemis arvensis L. subsp. acrochordona Briq. \& Cavill.

Anthemis arvensis $L$. subsp. arvensis

T Anthemis arvensis L. subsp. incrassata (Loisel.) Nyman

ET Anthemis arvensis L. subsp. sphacelata (C.Presl) R.Fern.

Anthemis chia L.

Anthemis cotula L.

ET Anthemis cretica L. subsp. alpina (L.) R.Fern.

E Anthemis cretica L. subsp. calabrica (Arcang.) R.Fern.

Anthemis cretica L. subsp. columnae (Ten.) Franzén

E Anthemis cretica L. subsp. messanensis (Brullo) Giardina \& Raimondo

E Anthemis cretica L. subsp. petraea (Ten.) Greuter

Anthemis cretica L. subsp. saxatilis (DC.) R.Fern.

E Anthemis cupaniana Tod. ex Nyman

E Anthemis hydruntina E.Groves subsp. hydruntina

ET Anthemis hydruntina E.Groves subsp. silensis (Fiori) Brullo, Gangale \& Uzunov

Anthemis ismelia Lojac.

Anthemis maritima $\mathrm{L}$. subsp. maritima

E Anthemis muricata (DC.) Guss.

Anthemis peregrina $\mathrm{L}$.

E Anthemis pignattiorum Guarino, Raimondo \& Domina

Anthemis secundiramea Biv.

Aposeris foetida (L.) Less.

Arctium lappa $\mathrm{L}$.

Arctium minus (Hill) Bernh.

Arctium nemorosum Lej.

Arctium tomentosum Mill.

Arnica montana L. subsp. montana

Arnoseris minima (L.) Schweigg. \& Körte

Artemisia absinthium $\mathrm{L}$.

Artemisia alba Turra

Artemisia arborescens (Vaill.) L.

Artemisia atrata Lam.

Artemisia caerulescens $L$. subsp. caerulescens

E Artemisia caerulescens L. subsp. cretacea (Fiori) Brilli-Catt. \& Gubellini

E Artemisia caerulescens L. subsp. densiflora (Viv.) Kerguélen \& Lambinon

Artemisia caerulescens L. subsp. gallica (Willd.) K.M.Perss. 
Artemisia campestris $\mathrm{L}$. subsp. alpina (DC.) Arcang.

Artemisia campestris L. subsp. borealis (Pall.) H.M.Hall \& Clem.

Artemisia campestris L. subsp. campestris

Artemisia campestris L. subsp. glutinosa (J.Gay ex Besser) Batt.

E Artemisia campestris $L$. subsp. variabilis (Ten.) Greuter

Artemisia chamaemelifolia Vill. subsp. chamaemelifolia

Artemisia genipi Weber ex Stechm.

Artemisia glacialis L.

Artemisia nitida Bertol.

Artemisia pedemontana Balb.

Artemisia umbelliformis Lam. subsp. eriantha (Ten.) Vallès-Xirau \& Oliva Brañas

Artemisia umbelliformis Lam. subsp. umbelliformis

Artemisia vallesiaca All.

Artemisia vulgaris $\mathrm{L}$.

Aster alpinus $\mathrm{L}$. subsp. alpinus

Aster amellus $\mathrm{L}$.

Asteriscus aquaticus (L.) Less.

Atractylis cancellata $\mathrm{L}$.

Bellidiastrum michelii Cass.

Bellis annua $L$. subsp. annua

$T \quad$ Bellis annua $L$. subsp. minuta (DC.) Meikle

E Bellis margaritifolia Huter, Porta \& Rigo

Bellis perennis $\mathrm{L}$.

Bellis sylvestris Cirillo

Bellium bellidioides $\mathrm{L}$.

E Bellium crassifolium Moris

Bellium minutum (L.) L.

Berardia lanuginosa (Lam.) Fiori

Bidens cernua $\mathrm{L}$.

Bidens tripartita L. subsp. bullata (L.) Rouy

Bidens tripartita L. subsp. tripartita

Bombycilaena discolor (Pers.) M.Laínz

Bombycilaena erecta (L.) Smoljan.

E Buphthalmum inuloides Moris

ET Buphthalmum salicifolium L. subsp. flexile (Bertol.) Garbari

Buphthalmum salicifolium $L$. subsp. salicifolium

Calendula arvensis (Vaill.) L.

Calendula stellata Cav.

Calendula suffruticosa Vahl subsp. fulgida (Raf.) Guadagno

Calendula suffruticosa Vahl subsp. maritima (Guss.) Meikle

Calendula suffruticosa Vahl subsp. suffruticosa

Calendula tripterocarpa Rupr.

Cardopatium corymbosum (L.) Pers.

Carduus acanthoides $\mathrm{L}$.

Carduus acicularis Bertol.

Carduus aemilii Briq. \& Cavill.

E Carduus affinis Guss. subsp. affinis

E Carduus affinis Guss. subsp. brutius (Fiori) Kazmi - Note: For the taxonomy and distribution of this subspecies, see Peruzzi et al. (2015d).

Carduus argyroa Biv.

Carduus carduelis (L.) Gren.

Carduus cephalanthus Viv.

Carduus chrysacanthus Ten.

Carduus collinus Waldst. \& Kit. subsp. collinus

Carduus collinus Waldst. \& Kit. subsp. cylindricus (Borbás) Soó

E Carduus corymbosus Ten.

Carduus crispus L. subsp. crispus

Carduus defloratus L. subsp. carlinifolius (Lam.) Ces.

Carduus defloratus $L$. subsp. defloratus

Carduus defloratus L. subsp. sumanus (Pollini) Arcang.

Carduus defloratus L. subsp. tridentinus (Evers) Ladurner

Carduus fasciculiflorus Viv.

Carduus litigiosus Nocca \& Balb. subsp. litigiosus

E Carduus membranaceus Lojac.

Carduus nigrescens Vill. subsp. nigrescens

T Carduus nutans L. subsp. alpicola (Gillot) Chass. \& Arènes

T Carduus nutans L. subsp. leiophyllus (Petrović) Stoj. \& Stef.

T Carduus nutans L. subsp. micropterus (Borbás) Hayek

Carduus nutans $L$. subsp. nutans

ET Carduus nutans L. subsp. perspinosus (Fiori) Arènes

$T \quad$ Carduus nutans L. subsp. platylepis (Rchb. \& Saut.) Nyman

T Carduus nutans $L$. subsp. scabrisquamus Arènes

ET Carduus nutans L. subsp. siculus (Franco) Greuter

T Carduus nutans L. subsp. taygeteus (Boiss. \& Heldr.) Hayek

Carduus personata (L.) Jacq. subsp. albidus (Adamović) Kazmi

Carduus personata (L.) Jacq. subsp. personata

ET Carduus pycnocephalus L. subsp. intermedius (Lojac.) Giardina \& Raimondo

$\mathrm{T} \quad$ Carduus pycnocephalus L. subsp. marmoratus (Boiss. \& Heldr.) P.H.Davis

Carduus pycnocephalus L. subsp. pycnocephalus 
Carduus sardous DC

Carduus tenuiflorus Curtis

Carlina acanthifolia All. subsp. acanthifolia

Carlina acanthifolia All. subsp. utzka (Hacq.) Meusel \& Kästner - Note: Extinct in Italy (Poldini et al. 2001).

Carlina acaulis L. subsp. acaulis

Carlina acaulis L. subsp. caulescens (Lam.) Schübl. \& G.Martens

Carlina biebersteinii Bernh. ex Hornem. subsp. biebersteinii

Carlina biebersteinii Bernh. ex Hornem. subsp. brevibracteata (Andrae) K.Werner

Carlina corymbosa $\mathrm{L}$.

Carlina gummifera (L.) Less.

E Carlina hispanica Lam. subsp. globosa (Arcang.) Meusel \& Kästner

Carlina involucrata Poir.

Carlina lanata L.

Carlina macrocephala Moris

Carlina nebrodensis Guss. ex DC.

Carlina racemosa $\mathrm{L}$.

Carlina sicula Ten. subsp. mareotica (Asch. \& Schweinf.) Greuter

Carlina sicula Ten. subsp. sicula

Carlina vulgaris $L$. subsp. spinosa (Velen.) Vandas

Carlina vulgaris $L$. subsp. vulgaris

Carpesium abrotanoides $\mathrm{L}$.

Carpesium cernuum $\mathrm{L}$.

Carthamus caeruleus $\mathrm{L}$.

Carthamus creticus $\mathrm{L}$.

Carthamus dentatus (Forssk.) Vahl

Carthamus lanatus L.

Carthamus pinnatus Desf.

E Castroviejoa frigida (Labill.) Galbany, L.Sáez \& Benedí - Note: Possibly exctinct.

E Castroviejoa montelinasana (Em.Schmid) Galbany, L.Sáez \& Benedí

Catananche caerulea $\mathrm{L}$.

Catananche lutea $\mathrm{L}$.

Centaurea aegusae Domina, Greuter \& Raimondo

Centaurea aeolica Guss. ex Lojac. subsp. aeolica

Centaurea aeolica Guss. ex Lojac. subsp. pandataria (Fiori \& Bég.) Anzal.

Centaurea aetaliae (Sommier) Bég.

Centaurea ambigua Guss. subsp. ambigua

Centaurea ambigua Guss. subsp. laciniata (Guss. ex DC.) Arcang.

Centaurea ambigua Guss. subsp. nigra (Fiori) Pignatti

Centaurea aplolepa Moretti subsp. aplolepa

Centaurea aplolepa Moretti subsp. bertolonii (Arrigoni) Greuter

Centaurea aplolepa Moretti subsp. carueliana (Micheletti) Dostál

Centaurea aplolepa Moretti subsp. cosana (Fiori) Dostál

Centaurea aplolepa Moretti subsp. gallinariae (Briq. \& Cavill.) Dostál

Centaurea aplolepa Moretti subsp. levantina (Arrigoni) Greuter

Centaurea aplolepa Moretti subsp. Iunensis (Fiori) Dostál

Centaurea aplolepa Moretti subsp. maremmana (Fiori) Dostál

Centaurea aplolepa Moretti subsp. parvula (Ces.) Arcang.

Centaurea aplolepa Moretti subsp. subciliata (DC.) Arcang.

Centaurea arachnoidea Viv. subsp. adonidifolia (Rchb.) F.Conti, Moraldo \& Ricceri

Centaurea arachnoidea Viv. subsp. arachnoidea

E Centaurea arachnoidea Viv. subsp. montis-ferrati Ricceri, Moraldo \& F.Conti

E Centaurea arrigonii Greuter

Centaurea aspera L. subsp. aspera

Centaurea aspromontana Brullo, Scelsi \& Spamp.

C Centaurea benedicta $(\mathrm{L}$.) $\mathrm{L}$.

ET Centaurea brulla Greuter

E Centaurea bugellensis (Soldano) Soldano

E Centaurea busambarensis Guss.

E Centaurea calabra G.Caruso, S.A.Giardina, Raimondo \& Spadaro

Centaurea calcitrapa L.

E Centaurea centauroides $\mathrm{L}$.

E Centaurea ceratophylla Ten. subsp. ceratophylla

E Centaurea cineraria L. subsp. cineraria

E Centaurea cineraria L. subsp. circae (Sommier) Cela Renz. \& Viegi

Centaurea collina L. subsp. collina

E Centaurea corensis Vals. \& Filigh.

Centaurea cristata Bartl.

Centaurea deusta Ten.

Centaurea dichroantha A.Kern.

E Centaurea diomedea Gasp.

E Centaurea erycina Raimondo \& Bancheva

E Centaurea filiformis Viv. subsp. ferulacea (Martelli) Arrigoni

E Centaurea filiformis Viv. subsp. filiformis

Centaurea flosculosa Willd.

E Centaurea giardinae Raimondo \& Spadaro

E Centaurea gussonei Raimondo \& Spadaro

E Centaurea gymnocarpa Moris \& De Not.

E Centaurea horrida Badarò 
E Centaurea iapygica (Lacaita) Brullo

E Centaurea ilvensis (Sommier) Arrigoni

ET Centaurea ionica Brullo

T Centaurea jacea L. subsp. angustifolia (DC.) Gremli

E Centaurea jacea L. subsp. forojulensis (Poldini) Greuter

Centaurea jacea L. subsp. gaudinii (Boiss. \& Reut.) Gremli

Centaurea jacea L. subsp. jacea

Centaurea jacea L. subsp. julica (Hayek) Greuter

Centaurea jacea L. subsp. weldeniana (Rchb.) Greuter

E Centaurea kartschiana Scop. subsp. kartschiana

ET Centaurea lacaitae Peruzzi - Note: The nomenclature and taxonomy of Centaurea tenorei Guss. ex Lacaita group provisionally follow Peruzzi (2008), waiting for further byosistematic studies (Santangelo et al. 2017).

E Centaurea leucadea Lacaita

ET Centaurea leucophaea Jord. subsp. brunnescens (Briq.) Dostál

Centaurea leucophaea Jord. subsp. leucophaea

E Centaurea litigiosa (Fiori) Arrigoni

E Centaurea macroacantha Guss.

Centaurea macroptilon Borbás

E Centaurea magistrorum Arrigoni \& Camarda

Centaurea melitensis $L$.

ET Centaurea montaltensis (Fiori) Peruzzi

Centaurea montana $\mathrm{L}$.

E Centaurea montis-borlae Soldano

Centaurea napifolia $\mathrm{L}$.

Centaurea nervosa Willd. subsp. nervosa

ET Centaurea nigrescens Willd. subsp. neapolitana (Boiss.) Dostál

Centaurea nigrescens Willd. subsp. nigrescens

ET Centaurea nigrescens Willd. subsp. pinnatifida (Fiori) Dostál

$\mathrm{T} \quad$ Centaurea nigrescens Willd. subsp. transalpina (Schleich. ex DC.) Nyman

ET Centaurea nobilis (E.Groves) Brullo

Centaurea paniculata L. subsp. paniculata

E Centaurea panormitana Lojac.

E Centaurea parlatoris Heldr. subsp. parlatoris

Centaurea parlatoris Heldr. subsp. virescens (Guss.) Raimondo \& Bancheva

Centaurea pentadactyli Brullo, Scelsi \& Spamp.

Centaurea poeltiana Puntillo

Centaurea pseudophrygia C.A.Mey.

Centaurea pumilio L.

Centaurea rhaetica Moritzi

Centaurea rupestris $\mathrm{L}$.

E Centaurea saccensis Raimondo, Bancheva \& llardi

E Centaurea sarfattiana Brullo, Gangale \& Uzunov

Centaurea scabiosa L. subsp. alpestris (Hegetschw.) Nyman

Centaurea scabiosa L. subsp. fritschii (Hayek) Hayek

Centaurea scabiosa L. subsp. grinensis (Reut.) Hayek

Centaurea scabiosa L. subsp. scabiosa

E Centaurea scannensis Anzal., Soldano \& F.Conti

ET Centaurea scillae Brullo

Centaurea seguenzae (Lacaita) Domina, Greuter \&

Raimondo,

Centaurea seridis L. subsp. sonchifolia (L.) Greuter

E Centaurea sicana Raimondo \& Spadaro

Centaurea sicula L.

$\mathrm{T} \quad$ Centaurea solstitialis L. subsp. adamii (Willd.) Nyman

T Centaurea solstitialis L. subsp. schouwii (DC.) Gugler

Centaurea solstitialis $L$. subsp. solstitialis

Centaurea sphaerocephala L. subsp. sphaerocephala

Centaurea stenolepis A.Kern. subsp. stenolepis

Centaurea stoebe L. - Note: For the taxonomy of this species, see Španiel et al. (2008).

E Centaurea subtilis Bertol.

E Centaurea tauromenitana Guss.

ET Centaurea tenacissima (E.Groves) Brullo

Centaurea tenoreana Willk.

Centaurea tenorei Guss. ex Lacaita

Centaurea todaroi Lacaita

Centaurea tommasinii A.Kern.

Centaurea triumfettii All. - Note: Several taxa belonging to Centaurea triumfettii group are recorded for Italy: C. triumfettii s.str., C. adscendens (Bartl.) Prodan, $C$. axillaris var. nana Ten. and C. graminifolia (Lam.) Muñoz Rodr. \& Devesa. Further studies are needed to understand the taxonomic value of these taxa and their distribution.

E Centaurea tyrrhena C.Brullo, Brullo \& Giusso

Centaurea uniflora Turra

Centaurea valesiaca (DC.) Jord.

E Centaurea veneris (Sommier) Bég.

Chamaemelum fuscatum (Brot.) Vasc.

Cheirolophus sempervirens (L.) Pomel

E Chiliadenus lopadusanus Brullo

Chondrilla chondrilloides (Ard.) H.Karst.

Chondrilla juncea $\mathrm{L}$. 
Cicerbita alpina (L.) Wallr.

Cichorium endivia L. subsp. pumilum (Jacq.) Cout.

Cichorium intybus L.

Cichorium spinosum $\mathrm{L}$.

Cirsium acaulon (L.) Scop. subsp. acaulon

Cirsium alpis-Iunae Brilli-Catt. \& Gubellini

Cirsium alsophilum (Pollini) Soldano

Cirsium arvense (L.) Scop.

E Cirsium bertolonii Spreng.

Cirsium canum (L.) All.

Cirsium carniolicum Scop. subsp. carniolicum

Cirsium creticum (Lam.) d'Urv. subsp. creticum

Cirsium creticum (Lam.) d'Urv. subsp. triumfettii (Lacaita) K.Werner

Cirsium echinatum (Desf.) DC.

Cirsium eriophorum (L.) Scop. subsp. eriophorum

Cirsium eriophorum (L.) Scop. subsp. spathulatum (Moretti) Ces.

Cirsium erisithales (Jacq.) Scop.

Cirsium ferox (L.) DC.

Cirsium heterophyllum (L.) Hill

Cirsium italicum DC.

E Cirsium lacaitae Petr.

E Cirsium lobelii Ten.

Cirsium monspessulanum (L.) Hill

Cirsium morisianum Rchb.f.

Cirsium oleraceum (L.) Scop.

Cirsium palustre (L.) Scop.

Cirsium pannonicum (L.f.) Link

Cirsium scabrum (Poir.) Bonnet \& Barratte

Cirsium spinosissimum (L.) Scop.

E Cirsium tenoreanum Petr.

Cirsium tuberosum (L.) All.

E Cirsium vallis-demonii Lojac.

$\mathrm{T} \quad$ Cirsium vulgare (Savi) Ten. subsp. crinitum (DC.) Arènes

$\mathrm{T} \quad$ Cirsium vulgare (Savi) Ten. subsp. silvaticum (Tausch) Arènes

Cirsium vulgare (Savi) Ten. subsp. vulgare

Cladanthus mixtus (L.) Chevall.

Coleostephus myconis (L.) Cass. ex Rchb.f.

Cota altissima (L.) J.Gay

Cota austriaca (Jacq.) Sch.Bip.

Cota segetalis (Ten.) Holub

T Cota tinctoria (L.) J.Gay subsp. australis (R.Fern.) Oberpr. \& Greuter

Cota tinctoria (L.) J.Gay subsp. tinctoria

Cota triumfettii (L.) J.Gay

Crepis albida Vill. subsp. albida

Crepis alpestris (Jacq.) Tausch

E Crepis apula (Fiori) Babc.

ET Crepis aspromontana Brullo, Scelsi \& Spamp.

Crepis aurea (L.) Cass. subsp. aurea

Crepis aurea (L.) Cass. subsp. glabrescens (Caruel) Arcang.

Crepis bellidifolia Loisel.

Crepis biennis $\mathrm{L}$.

Crepis bursifolia L.

Crepis capillaris (L.) Wallr.

Crepis chondrilloides Jacq.

Crepis conyzifolia (Gouan) A.Kern.

Crepis foetida $L$. subsp. foetida

Crepis froelichiana DC. ex Froel. subsp. dinarica (Beck) Gutermann

Crepis froelichiana DC. ex Froel. subsp. froelichiana

Crepis jacquinii Tausch subsp. kerneri (Rech.f.) Merxm.

Crepis lacera Ten. subsp. Iacera

ET Crepis lacera Ten. subsp. titani (Pamp.) Roma-Marzio, G.Astuti \& Peruzzi - Note: For the taxonomy and distribution of this subspecies, see Roma-Marzio et al. (2015).

Crepis leontodontoides All.

E Crepis magellensis F.Conti \& Uzunov - Note: For the taxonomy and distribution of this species, see Conti and Uzunov (2011).

Crepis mollis (Jacq.) Asch. subsp. mollis

Crepis mollis (Jacq.) Asch. subsp. succisifolia (All.) Dostál

Crepis neglecta L. subsp. corymbosa (Ten.) Nyman

Crepis neglecta L. subsp. neglecta

Crepis nicaeensis Balb.

Crepis paludosa (L.) Moench

Crepis pontana (L.) Dalla Torre

Crepis praemorsa (L.) Walther

Crepis pulchra L. subsp. pulchra

Crepis pygmaea $\mathrm{L}$.

Crepis pyrenaica (L.) Greuter

Crepis rhaetica Hegetschw.

Crepis rubra $\mathrm{L}$.

Crepis setosa Haller $\mathrm{f}$ 
Crepis sprengelii Nicotra

Crepis suffreniana (DC.) Steud.

Crepis tectorum $\mathrm{L}$.

Crepis terglouensis (Hacq.) A.Kern.

ET Crepis vesicaria L. subsp. bivonana (Soldano \& F.Conti) Giardina \& Raimondo

ET Crepis vesicaria L. subsp. hyemalis (Biv.) Babc.

T Crepis vesicaria L. subsp. taraxacifolia (Thuill.) Thell.

Crepis vesicaria $L$. subsp. vesicaria

Crepis zacintha (L.) Loisel.

Crupina crupinastrum (Moris) Vis.

Crupina intermedia (Mutel) Walp.

Crupina vulgaris Cass.

Cynara cardunculus L. subsp. cardunculus

C Cynara cardunculus L. subsp. flavescens Wiklund - Note: This taxon may be a feral of the crop cardoon (C. cardunculus L. var. altilis DC.) (Gatto et al. 2013).

ET Cynara cardunculus L. subsp. zingaroensis (Raimondo \& Domina) Raimondo \& Domina

Dittrichia graveolens (L.) Greuter

Dittrichia viscosa (L.) Greuter subsp. viscosa

Doronicum austriacum Jacq. subsp. austriacum

Doronicum clusii (All.) Tausch

Doronicum columnae Ten. - Note: Hybrids among this species and D. orientale (D. ×minutilloi Peruzzi) are known from the Aurunci Mts., LAZ (Peruzzi et al. 2012b).

Doronicum glaciale (Wulfen) Nyman subsp. glaciale

Doronicum grandiflorum Lam. subsp. grandiflorum

Doronicum orientale Hoffm.

Doronicum pardalianches $\mathrm{L}$.

Doronicum plantagineum L.

Echinops exaltatus Schrad.

Echinops ritro L. - Note: Echinops ritro L. subsp. ritro and E. ritro subsp. ruthenicus (M.Bieb.) Nyman are both recorded for Italy. However, considering that the name $E$. ritro is not yet typified, we are not able to safely recognize any subspecies in Italy.

E Echinops siculus Strobl

T Echinops sphaerocephalus L. subsp. albidus (Boiss. \& Spruner) Kožuharov

Echinops sphaerocephalus L. subsp. sphaerocephalus

T Echinops spinosissimus Turra subsp. neumayeri (Vis.) Kožuharov

Echinops spinosissimus Turra subsp. spinosissimus

T Echinops spinosissimus Turra subsp. spinosus Greuter

Erigeron acris L. subsp. acris

Erigeron acris L. subsp. angulosus (Gaudin) Vacc.

Erigeron acris L. subsp. serotinus (Weihe) Greuter

Erigeron alpinus $\mathrm{L}$.

Erigeron atticus Vill.

Erigeron epiroticus (Vierh.) Halácsy

Erigeron glabratus Hoppe \& Hornsch. ex Bluff \& Fingerh.

Erigeron neglectus A.Kern.

Erigeron schleicheri Gremli

Erigeron uniflorus L.

Eupatorium cannabinum L. subsp. cannabinum

T Eupatorium cannabinum L. subsp. corsicum (Req. ex Loisel.) P.Fourn.

Evacidium discolor (DC.) Maire

Filago arvensis $\mathrm{L}$.

Filago asterisciflora (Lam.) Sweet

Filago congesta Guss. ex DC.

Filago eriocephala Guss.

Filago germanica (L.) Huds.

Filago lutescens Jord. subsp. Iutescens

Filago neglecta (Soy.-Will.) DC.

Filago pygmaea $\mathrm{L}$.

Filago pyramidata $\mathrm{L}$.

E Filago tyrrhenica Chrtek \& Holub

Galactites tomentosus Moench

Galatella linosyris (L.) Rchb.f. subsp. linosyris

Galatella pannonica (Jacq.) Galasso, Bartolucci \& Ardenghi subsp. pannonica

Galatella sedifolia (L.) Greuter subsp. sedifolia

Geropogon hybridus (L.) Sch.Bip.

Glebionis coronaria (L.) Spach

T Glebionis discolor (d'Urv.) E.Cano, Musarella, Cano-Ortiz, Piñar Fuentes, Spamp. \& Pinto Gomes

Glebionis segetum (L.) Fourr.

Gnaphalium uliginosum $\mathrm{L}$.

Hedypnois rhagadioloides (L.) F.W.Schmidt

E Helichrysum errerae Tineo

Helichrysum italicum (Roth) G.Don subsp. italicum

E Helichrysum italicum (Roth) G.Don subsp. pseudolitoreum (Fiori) Bacch., Brullo \& Mossa

Helichrysum italicum (Roth) G.Don subsp. siculum (Jord. \& Fourr.) Galbany, L.Sáez \& Benedí

Helichrysum italicum (Roth) G.Don subsp. tyrrhenicum (Bacch., Brullo \& Giusso) Herrando, J.M.Blanco, L.Sáez Galbany

E Helichrysum litoreum Guss.

Helichrysum Iuteoalbum (L.) Rchb.

Helichrysum pendulum (C.Presl) C.Presl - Note: According to Herrando-Moraira et al. (2017), H. hyblaeum Brullo, H. nebrodense Heldr. and H. panormitanum

Tineo ex Guss. are synonyms of $H$. pendulum. A different taxonomic setting, not followed here, was recently proposed by lamonico et al. (2016b).

Helichrysum saxatile Moris subsp. morisianum Bacch., Brullo \& Mossa 
Helichrysum saxatile Moris subsp. saxatile

Helichrysum stoechas (L.) Moench subsp. barrelieri (Ten.) Nyman

Helichrysum stoechas (L.) Moench subsp. stoechas

Helminthotheca aculeata (Vahl) Lack subsp. aculeata

Helminthotheca echioides (L.) Holub

Hieracium acanthodontoides Arv-Touv. \& Belli

Hieracium adenophyton (Zahn) Zahn

Hieracium alpinum L. subsp. alpinum

Hieracium alpinum L. subsp. halleri (Vill.) Ces.

E Hieracium alpinum L. subsp. marcelli Paléz. \& Zahn

E Hieracium alpinum L. subsp. ormeanum Zahn

E Hieracium amphisericophorum Zahn

Hieracium amplexicaule L. subsp. amplexicaule

E Hieracium amplexicaule $L$. subsp. berardianoides Zahn

Hieracium amplexicaule L. subsp. berardianum (Arv.-Touv.) Zahn

Hieracium amplexicaule L. subsp. cadinense (Evers) Zahn

Hieracium amplexicaule L. subsp. chenevardianum Zahn

Hieracium amplexicaule L. subsp. neopetraeum Zahn

Hieracium amplexicaule L. subsp. pseudocerinthoides (Arv.-Touv.) Zahn

Hieracium amplexicaule L. subsp. pseudoligusticum (Gremli) Zahn

Hieracium amplexicaule L. subsp. pulmonarioides (Vill.) Ces.

Hieracium amplexicaule L. subsp. spelaeum (Arv.-Touv.) Zahn

E Hieracium annae-toutoniae Zahn subsp. vilpianense Gottschl.

E Hieracium antholzense Zahn

Hieracium aphyllum Nägeli \& Peter subsp. hololeucum (Arv.-Touv. \& A. Faure) Zahn

E Hieracium aphyllum Nägeli \& Peter subsp. singulare (Huter ex Zahn) Zahn

E Hieracium apricorum Wiesb. ex Dichtl subsp. pseudolonganum (Zahn) Greuter Hieracium apricorum Wiesb. ex Dichtl subsp. sublaevigatum (Beck) Gottschl.

Hieracium apricorum Wiesb. ex Dichtl subsp. subpallescens (Beck) Gottschl.

E Hieracium apricorum Wiesb. ex Dichtl subsp. valdulcense Gottschl.

E Hieracium argothrix Nägeli \& Peter subsp. anisodon Nägeli \& Peter

Hieracium argothrix Nägeli \& Peter subsp. argothrix

E Hieracium argothrix Nägeli \& Peter subsp. candidulum Nägeli \& Peter

E Hieracium argothrix Nägeli \& Peter subsp. pleiobrachion Zahn

Hieracium armerioides Arv.-Touv. subsp. absconditum (Huter) Zahn

Hieracium armerioides Arv.-Touv. subsp. anadenum (Burnat \& Gremli) Zahn

Hieracium armerioides Arv.-Touv. subsp. armerioides

E Hieracium armerioides Arv.-Touv. subsp. collae Zahn

Hieracium armerioides Arv.-Touv. subsp. crispulum (Nägeli \& Peter) Zahn

Hieracium armerioides Arv.-Touv. subsp. favreanum (Arv.-Touv.) Zahn

Hieracium armerioides Arv.-Touv. subsp. leucochlorum (Arv.-Touv.) Zahn

Hieracium armerioides Arv.-Touv. subsp. nigritellum (Arv.-Touv.) Zahn

E Hieracium armerioides Arv.-Touv. subsp. paucitrichum Gottschl.

Hieracium armerioides Arv.-Touv. subsp. phalacrophyllum (Nägeli \& Peter) Zahn

Hieracium armerioides Arv.-Touv. subsp. rhombophyllum (Nägeli \& Peter) Zahn

E Hieracium armerioides Arv.-Touv. subsp. seirasicum (Zahn) Zahn

E Hieracium armerioides Arv.-Touv. subsp. siphonophorum Wilczek \& Zahn

Hieracium armerioides Arv.-Touv. subsp. touvetii (Nägeli \& Peter) Greuter

Hieracium armerioides Arv.-Touv. subsp. ustulatum (Arv.-Touv.) Zahn

Hieracium arolae (Murr) Zahn subsp. arolae

E Hieracium arolae (Murr) Zahn subsp. macrocalathium Zahn

E Hieracium arolae (Murr) Zahn subsp. rhoeadifolioides Hayek \& Zahn

E Hieracium arpadianum Zahn subsp. pugnaculum Gottschl.

Hieracium arpadianum Zahn subsp. sellanum Zahn

Hieracium arpadianum Zahn subsp. subscariolaceum Zahn

E Hieracium aspromontanum Brullo, Scelsi \& Spamp.

Hieracium atratum Fr. subsp. atratum

Hieracium atratum Fr. subsp. dolichaetoides Zahn

E Hieracium atratum Fr. subsp. neohayekii Zahn

Hieracium atratum Fr. subsp. pseudoleicolor Fen. \& Zahn

Hieracium atratum Fr. subsp. squalidiforme (Murr \& Zahn) Zahn

Hieracium atratum Fr. subsp. subnigrescens (Norrl.) Zahn

Hieracium atratum Fr. subsp. tauriscorum Zahn

E Hieracium atratum Fr. subsp. zermulae Gottschl.

E Hieracium atrocalyx Gottschl.

E Hieracium australe Fr. subsp. australe

Hieracium australe Fr. subsp. insuetum (Jord. ex Boreau) Fen. \& Zahn

Hieracium balbisianum Arv.-Touv. \& Briq. subsp. balbisianum

Hieracium balbisianum Arv.-Touv. \& Briq. subsp. issense (Murr \& Zahn) Greuter

Hieracium balbisianum Arv.-Touv. \& Briq. subsp. kerneri (Ausserd. ex Zahn) Greuter

Hieracium barbulare Zahn subsp. barbulare

E Hieracium barrelieri Gottschl., Raimondo, Greuter \& Di Grist.

Hieracium bastrerianum Zahn subsp. bastrerianum

E Hieracium bastrerianum Zahn subsp. pramolicum Zahn

Hieracium benzianum Murr \& Zahn subsp. benzianum

Hieracium benzianum Murr \& Zahn subsp. ctenodontiforme Zahn \& Benz

Hieracium benzianum Murr \& Zahn subsp. ctenodontoides (Zahn) Zahn

E Hieracium benzianum Murr \& Zahn subsp. pseudobenzianum (Dalla Torre \& Sarnth.) Zahn 
Hieracium benzianum Murr \& Zahn subsp. sciaphiloides (Zahn) Greuter Hieracium bernardii Rouy subsp. bernardii

E Hieracium bernardii Rouy subsp. gallurense (Arrigoni) Greuter

E Hieracium bernardii Rouy subsp. templare (Arrigoni) Greuter

E Hieracium beyeri Zahn

Hieracium bicknellianum Belli \& Arv.-Touv.

Hieracium bifidum Kit. ex Hornem. subsp. amoenobium Zahn

Hieracium bifidum Kit. ex Hornem. subsp. basicuneatifolium (Touton ex Dalla Torre \& Sarnth.) Müll.Dornst.

Hieracium bifidum Kit. ex Hornem. subsp. basicuneatum (Zahn) Zahn

E Hieracium bifidum Kit. ex Hornem. subsp. bifidipraecox Fen. \& Zahn

E Hieracium bifidum Kit. ex Hornem. subsp. bolognense Zahn

Hieracium bifidum Kit. ex Hornem. subsp. caesiiflorum (Norrl.) Zahn

E Hieracium bifidum Kit. ex Hornem. subsp. caesioprasinum Zahn

Hieracium bifidum Kit. ex Hornem. subsp. canitiosum (Malme) Zahn

Hieracium bifidum Kit. ex Hornem. subsp. cardiobasis Zahn

E Hieracium bifidum Kit. ex Hornem. subsp. condylisquamum Gottschl.

Hieracium bifidum Kit. ex Hornem. subsp. cordigenum (Norrl.) Zahn

Hieracium bifidum Kit. ex Hornem. subsp. coriifolium (Arv.-Touv.) Zahn

Hieracium bifidum Kit. ex Hornem. subsp. dolomiticum (Willk.) Dalla Torre \& Sarnth.

E Hieracium bifidum Kit. ex Hornem. subsp. elvense Gottschl. \& Dunkel

Hieracium bifidum Kit. ex Hornem. subsp. eriopodoides (Zahn) Zahn

Hieracium bifidum Kit. ex Hornem. subsp. erucoides (Arv.-Touv.) Zahn

Hieracium bifidum Kit. ex Hornem. subsp. fenarolii P.Rossi \& Zahn

E Hieracium bifidum Kit. ex Hornem. subsp. gardetanum P.Rossi \& Zahn

Hieracium bifidum Kit. ex Hornem. subsp. laceridens (Zahn) Zahn

Hieracium bifidum Kit. ex Hornem. subsp. Iaciniare Zahn

Hieracium bifidum Kit. ex Hornem. subsp. lepidum (Arv.-Touv.) Zahn

E Hieracium bifidum Kit. ex Hornem. subsp. megalocalathium P.Rossi \& Zahn

Hieracium bifidum Kit. ex Hornem. subsp. megalotomum (Zahn) Zahn

E Hieracium bifidum Kit. ex Hornem. subsp. mollinense Zahn

E Hieracium bifidum Kit. ex Hornem. subsp. nummulariifolium Gottschl.

Hieracium bifidum Kit. ex Hornem. subsp. obscurisquamum (Zahn) Greuter

Hieracium bifidum Kit. ex Hornem. subsp. oreites (Arv.-Touv.) Zahn

Hieracium bifidum Kit. ex Hornem. subsp. oreitiforme (Zahn) Zahn

Hieracium bifidum Kit. ex Hornem. subsp. perlacerum Zahn

Hieracium bifidum Kit. ex Hornem. subsp. poliomelanum Zahn

Hieracium bifidum Kit. ex Hornem. subsp. pourriacense Zahn

Hieracium bifidum Kit. ex Hornem. subsp. prasinescens Zahn

Hieracium bifidum Kit. ex Hornem. subsp. psammogenes (Zahn) Zahn

Hieracium bifidum Kit. ex Hornem. subsp. pseudobasicuneatum (Touton ex Dalla Torre \& Sarnth.) Zahn

Hieracium bifidum Kit. ex Hornem. subsp. pseudodollineri (Murr \& Zahn) Zahn

Hieracium bifidum Kit. ex Hornem. subsp. pseudoligocephalum (Zahn) Zahn

Hieracium bifidum Kit. ex Hornem. subsp. pseudopraecox (Zahn) Dalla Torre \& Sarnth.

Hieracium bifidum Kit. ex Hornem. subsp. pseudopsammogenes (Dalla Torre \& Sarnth.) Zahn

E Hieracium bifidum Kit. ex Hornem. subsp. pseudosemisilvaticum (Dalla Torre \& Sarnth.) Zahn

Hieracium bifidum Kit. ex Hornem. subsp. sagittibasis Zahn

Hieracium bifidum Kit. ex Hornem. subsp. scandinaviorum Zahn

Hieracium bifidum Kit. ex Hornem. subsp. scutatum (Arv.-Touv.) Zahn

E Hieracium bifidum Kit. ex Hornem. subsp. semipraecox P.Rossi \& Zahn

E Hieracium bifidum Kit. ex Hornem. subsp. semitenuiflorum Fen. \& Zahn

Hieracium bifidum Kit. ex Hornem. subsp. senile (Arv.-Touv.) Zahn

Hieracium bifidum Kit. ex Hornem. subsp. seniliforme (Zahn) Zahn

E Hieracium bifidum Kit. ex Hornem. subsp. serpentinicolor Fen. \& Zahn

Hieracium bifidum Kit. ex Hornem. subsp. sinuosifrons (Dahlst.) Zahn

Hieracium bifidum Kit. ex Hornem. subsp. sivyense Zahn

Hieracium bifidum Kit. ex Hornem. subsp. stenolepis (Lindeb.) Zahn

Hieracium bifidum Kit. ex Hornem. subsp. subcaesiifloriforme (Zahn) Zahn

Hieracium bifidum Kit. ex Hornem. subsp. subcanescentiforme Käser

E Hieracium bifidum Kit. ex Hornem. subsp. subhastatum Gottschl.

E Hieracium bifidum Kit. ex Hornem. subsp. subimbricatum Gottschl.

Hieracium bifidum Kit. ex Hornem. subsp. submolliceps Zahn

Hieracium bifidum Kit. ex Hornem. subsp. subpapyraceum Zahn ex Chiarugi

Hieracium bifidum Kit. ex Hornem. subsp. subsagittatum (Zahn) Zahn

Hieracium bifidum Kit. ex Hornem. subsp. subtenuiflorum (Zahn) Greuter

Hieracium bifidum Kit. ex Hornem. subsp. taraxacifolium Zahn

Hieracium bifidum Kit. ex Hornem. subsp. toutonii Zahn

E Hieracium bifidum Kit. ex Hornem. subsp. vaccarii Besse \& Zahn

Hieracium bifidum Kit. ex Hornem. subsp. vensicum Zahn

Hieracium bocconei Griseb. subsp. bocconei

Hieracium bocconei Griseb. subsp. devexicola Zahn

Hieracium bocconei Griseb. subsp. engadinense (Zahn) Zahn

Hieracium bocconei Griseb. subsp. ramiparum Zahn

Hieracium borealiforme P.D.Sell \& C.West

E Hieracium boreoapenninum Gottschl.

E Hieracium bornetii Burnat \& Gremli

E Hieracium brevifolium Tausch subsp. bergameum Zahn

Hieracium brevifolium Tausch subsp. brevifolium

Hieracium brevifolium Tausch subsp. caruelii Zahn 
Hieracium brevifolium Tausch subsp. cesatianum (Zahn) Gottschl.

Hieracium brevifolium Tausch subsp. halimifolium (Froel.) Zahn

Hieracium brevifolium Tausch subsp. hellwegeri (Murr \& Zahn) Zahn

Hieracium brevifolium Tausch subsp. lombardense Gottschl.

Hieracium brevifolium Tausch subsp. muraltae (Zahn) Greuter

Hieracium brevifolium Tausch subsp. virgaureiforme Zahn

Hieracium brillii Gottschl.

Hieracium bugellense Gottschl.

Hieracium bupleuroides C.C.Gmel. subsp. adenobupleuroides Fen. \& Zahn

Hieracium bupleuroides C.C.Gmel. subsp. aprutiorum (Furrer \& Zahn) Gottschl.

Hieracium bupleuroides C.C.Gmel. subsp. bupleuroides

E Hieracium bupleuroides C.C.Gmel. subsp. cassianum Pamp. \& Zahn

Hieracium bupleuroides C.C.Gmel. subsp. comophyllum Nägeli \& Peter

Hieracium bupleuroides C.C.Gmel. subsp. crinifolium Nägeli \& Peter

Hieracium bupleuroides C.C.Gmel. subsp. eriolepium Nägeli \& Peter

Hieracium bupleuroides C.C.Gmel. subsp. Iaeviceps Nägeli \& Peter

E Hieracium bupleuroides C.C.Gmel. subsp. mnoolepium Nägeli \& Peter

E Hieracium bupleuroides C.C.Gmel. subsp. moenense Zahn

Hieracium bupleuroides C.C.Gmel. subsp. phyllobracteum Nägeli \& Peter

E Hieracium bupleuroides C.C.Gmel. subsp. praetutiense Nägeli \& Peter

E Hieracium bupleuroides C.C.Gmel. subsp. romanorum Korb \& Zahn

Hieracium bupleuroides C.C.Gmel. subsp. scabriceps Nägeli \& Peter

Hieracium bupleuroides C.C.Gmel. subsp. schenkii (Griseb.) Nägeli \& Peter

Hieracium bupleuroides C.C.Gmel. subsp. tririvicola Gottsch.

Hieracium burnatii Arv.-Touv. subsp. burnatii

Hieracium burnatii Arv.-Touv. subsp. tomentellum (Nägeli \& Peter) Zahn

E Hieracium busambarense Caldarella, Gianguzzi \& Gottschl.

Hieracium cabreranum Arv.-Touv.

Hieracium caesioides Arv.-Touv. subsp. aleuroclinum Zahn

Hieracium caesioides Arv.-Touv. subsp. bordigherae (Zahn) Zahn

Hieracium caesioides Arv.-Touv. subsp. caesioides

Hieracium caesioides Arv.-Touv. subsp. diamantense Zahn

Hieracium caesioides Arv.-Touv. subsp. liguricola Zahn

Hieracium caesioides Arv.-Touv. subsp. lobophorum Zahn

Hieracium caesioides Arv.-Touv. subsp. lobophyton Zahn

Hieracium caesioides Arv.-Touv. subsp. pectinatifrons Zahn

Hieracium caesioides Arv.-Touv. subsp. prasinops (Touton \& Zahn) Zahn

Hieracium caesioides Arv.-Touv. subsp. rionii (Gremli) Zahn

E Hieracium caesioides Arv.-Touv. subsp. supraglauciforme Zahn

Hieracium caesioides Arv.-Touv. subsp. supraglaucum (C.Bicknell \& Zahn) Zahn

Hieracium caesium (Fr.) Fr. subsp. brennerianum (Zahn) Gottschl.

Hieracium caesium (Fr.) Fr. subsp. caesiopsis Zahn

Hieracium caesium (Fr.) Fr. subsp. laeticolor Almq.

Hieracium caesium (Fr.) Fr. subsp. lentiginosum (Murr \& Zahn) Zahn

Hieracium caesium (Fr.) Fr. subsp. sendtneri (Nägeli ex Gremli) Vollm.

Hieracium caesium ( $\mathrm{Fr}$.) Fr. subsp. vennaevallis Zahn

Hieracium caesium (Fr.) Fr. subsp. virenticeps (Dahlst.) Zahn

Hieracium calcareum Bernh. ex Hornem. subsp. baldense (Nägeli \& Peter) Greuter

Hieracium calcareum Bernh. ex Hornem. subsp. baldensiforme (Nägeli \& Peter) Greuter

Hieracium calcareum Bernh. ex Hornem. subsp. calcareum

Hieracium calcareum Bernh. ex Hornem. subsp. carnicum (Nägeli \& Peter) Greuter

Hieracium calcareum Bernh. ex Hornem. subsp. crinopodum (Nägeli \& Peter) Greuter

Hieracium calcareum Bernh. ex Hornem. subsp. feliciense (Nägeli \& Peter) Greuter

Hieracium calcareum Bernh. ex Hornem. subsp. glauciforme (Nägeli \& Peter) Greuter

Hieracium calcareum Bernh. ex Hornem. subsp. holleri (Nägeli \& Peter) Greuter

Hieracium calcareum Bernh. ex Hornem. subsp. illyricum (Fr.) Greuter

Hieracium calcareum Bernh. ex Hornem. subsp. papperitzii (Rchb.f.) Greuter

Hieracium calcareum Bernh. ex Hornem. subsp. pseudothyrsodes (Zahn) Greuter

E Hieracium calcareum Bernh. ex Hornem. subsp. trilacense (Murr) Greuter

Hieracium calocymum Zahn subsp. calocymum

E Hieracium calothyrsum Zahn ex Murr

E Hieracium carpegnae Gottschl.

E Hieracium cavallense Gottschl.

Hieracium cavillieri Zahn subsp. cavillieri

Hieracium cavillieri Zahn subsp. vesubiense Zahn

Hieracium cephalotes Arv.-Touv. subsp. cephalotes

Hieracium chiariglionei Gottschl.

Hieracium chlorelloides Zahn

Hieracium chlorifolium Arv.-Touv. subsp. chlorifolium

Hieracium chlorifolium Arv.-Touv. subsp. fulcratiforme Zahn

Hieracium chlorifolium Arv.-Touv. subsp. isoplates (Nägeli \& Peter) Zahn

Hieracium chlorifolium Arv.-Touv. subsp. leoninum (Nägeli \& Peter) Zahn

Hieracium chlorifolium Arv.-Touv. subsp. pseudopenninum Zahn

Hieracium chlorifolium Arv.-Touv. subsp. pulchriforme Murr \& Zahn

Hieracium chlorifolium Arv.-Touv. subsp. rendinaricum Gottschl.

Hieracium chlorophyton Preissm. \& Zahn ex Hayek subsp. Ianguidum (Zahn) Greuter 
Hieracium chloropsis Gren. \& Godr. subsp. chloropsis

$\mathrm{E}$

Hieracium chloropsis Gren. \& Godr. subsp. erioleion (Nägeli \& Peter) Zahn

Hieracium chloropsis Gren. \& Godr. subsp. gerardii (Arv.-Touv. ex Legrand) Zahn

Hieracium chloropsis Gren. \& Godr. subsp. gnaphalodes (Nägeli \& Peter) Zahn

Hieracium chondrillifolium Fr. subsp. boissieri (A.Huet \& É.Huet ex Arv.-Touv.) Zahn

Hieracium chondrillifolium Fr. subsp. calcicola (Nägeli \& Peter) Zahn

Hieracium chondrillifolium Fr. subsp. casterinense (Zahn) Zahn

E Hieracium chondrillifolium Fr. subsp. chiarugianum Zahn ex Chiarugi

Hieracium chondrillifolium Fr. subsp. chondrillifolium

Hieracium chondrillifolium Fr. subsp. comolepium (Nägeli \& Peter) Zahn

Hieracium chondrillifolium Fr. subsp. delasoiei (Lagger ex De la Soie) Zahn

Hieracium chondrillifolium Fr. subsp. doliocephalum (Nägeli \& Peter) Zahn

Hieracium chondrillifolium Fr. subsp. megalocladum (Nägeli \& Peter) Zahn

E Hieracium chondrillifolium Fr. subsp. morascanum (Zahn) Zahn

E Hieracium chondrillifolium Fr. subsp. otenicum Pamp. \& Zahn

Hieracium chondrillifolium Fr. subsp. patulum (Nägeli \& Peter) Zahn

Hieracium chondrillifolium Fr. subsp. supracalvum (Nägeli \& Peter) Zahn

E Hieracium cirritogenes Zahn

Hieracium cirritum Arv.-Touv. subsp. amphiglaucum Zahn

Hieracium cirritum Arv.-Touv. subsp. biceps (Zahn) Zahn

E Hieracium cirritum Arv.-Touv. subsp. brentanum (Touton ex Dalla Torre \& Sarnth.) Zahn

Hieracium cirritum Arv.-Touv. subsp. cirritum

Hieracium cirritum Arv.-Touv. subsp. elisum (Arv.-Touv.) Nägeli \& Peter

Hieracium cirritum Arv.-Touv. subsp. hypochaerideum (Arv.-Touv.) Nägeli \& Peter

Hieracium cirritum Arv.-Touv. subsp. hypochoeridiforme (Zahn) Zahn

Hieracium cirritum Arv.-Touv. subsp. permirum (Zahn) Zahn

Hieracium cirritum Arv.-Touv. subsp. pravum Zahn

Hieracium cirritum Arv.-Touv. subsp. pseudocirritum (Zahn \& Touton ex Favre) Zahn

E Hieracium cirritum Arv.-Touv. subsp. pseudocochleare Gottschl.

Hieracium cirritum Arv.-Touv. subsp. tenellum (Huter \& Ausserd.) Dalla Torre \& Sarnth.

E Hieracium cirsiopsis Gottschl. \& Dunkel

E Hieracium contii Gottschl.

E Hieracium cophanense Lojac.

E Hieracium cornuscalae Gottschl.

Hieracium coronariifolium Arv.-Touv.

Hieracium cottetii Godet ex Christener subsp. cottetii

Hieracium crocatum Fr. subsp. conicum (Arv.-Touv.) Murr \& Zahn

Hieracium cryptadenum Arv.-Touv. subsp. cryptadenum

Hieracium cryptadenum Arv.-Touv. subsp. megalodon (Nägeli \& Peter) Zahn

Hieracium ctenodon Nägeli \& Peter subsp. ctenodon

Hieracium ctenodon Nägeli \& Peter subsp. multiramum Nägeli \& Peter

Hieracium ctenodon Nägeli \& Peter subsp. zahnii (Oborny) Murr \& Zahn

Hieracium cydoniifolium Vill. subsp. brassicoides (Wilczek) Zahn

Hieracium cydoniifolium Vill. subsp. cottianum (Arv.-Touv.) Zahn

E Hieracium cydoniifolium Vill. subsp. isatidiforme Zahn

Hieracium cydoniifolium Vill. subsp. mespilifolium (Arv.-Touv.) Zahn

Hieracium cydoniifolium Vill. subsp. ochroleucomorphum Zahn

Hieracium cydoniifolium Vill. subsp. parcepilosum (Arv.-Touv.) Zahn

Hieracium cydoniifolium Vill. subsp. prenanthophyllum (Nägeli \& Peter) Zahn

Hieracium cydoniifolium Vill. subsp. semiperfoliatum Zahn

Hieracium cydoniifolium Vill. subsp. subpanduratum Zahn

Hieracium cydoniifolium Vill. subsp. sulgeri (Murr) Zahn

E Hieracium cydoniifolium Vill. subsp. trichodes (Rchb.f.) Zahn

Hieracium cydoniifolium Vill. subsp. trichojuranum (Zahn) Zahn

Hieracium dasytrichum Arv.-Touv. subsp. capnoides (Kern. ex Näg. \& Peter) Zahn

Hieracium dasytrichum Arv.-Touv. subsp. dasytrichum

Hieracium dasytrichum Arv.-Touv. subsp. mesomorphum (Nägeli \& Peter) Zahn

Hieracium dasytrichum Arv.-Touv. subsp. neopolytrichum (Arv.-Touv.) Greuter

Hieracium dasytrichum Arv.-Touv. subsp. pseudopiliferum (Nägeli \& Peter) Zahn

Hieracium dasytrichum Arv.-Touv. subsp. subpiliferum (Arv.-Touv.) Zahn

Hieracium dentatum Hoppe subsp. aechmetes Nägeli \& Peter

Hieracium dentatum Hoppe subsp. basifoliatum Nägeli \& Peter

Hieracium dentatum Hoppe subsp. carinthicola Nägeli \& Peter

Hieracium dentatum Hoppe subsp. cenisium (Arv.-Touv.) Zahn

Hieracium dentatum Hoppe subsp. dentatifolium Nägeli \& Peter

Hieracium dentatum Hoppe subsp. dentatiforme Nägeli \& Peter

Hieracium dentatum Hoppe subsp. dentatum

E Hieracium dentatum Hoppe subsp. dentissimum Gottschl.

Hieracium dentatum Hoppe subsp. depressum Nägeli \& Peter

Hieracium dentatum Hoppe subsp. erigerontinum (Arv.-Touv.) Zahn

Hieracium dentatum Hoppe subsp. expallens (Fr.) Nägeli \& Peter

Hieracium dentatum Hoppe subsp. gaudinii (Christener) Nägeli \& Peter

Hieracium dentatum Hoppe subsp. Ionchites Nägeli \& Peter

Hieracium dentatum Hoppe subsp. maculifolium Nägeli \& Peter

Hieracium dentatum Hoppe subsp. marmoratum Nägeli \& Peter

E Hieracium dentatum Hoppe subsp. montiscatriae Gottschl.

Hieracium dentatum Hoppe subsp. oblongifolium Nägeli \& Peter

Hieracium dentatum Hoppe subsp. ormontense Nägeli \& Peter 
Hieracium dentatum Hoppe subsp. prionodes Nägeli \& Peter

Hieracium dentatum Hoppe subsp. pseudoplantagineum (Zahn) Zahn

Hieracium dentatum Hoppe subsp. pseudoporrectum (Gremli) Nägeli \& Peter

Hieracium dentatum Hoppe subsp. pseudovillosum Nägeli \& Peter

Hieracium dentatum Hoppe subsp. setuligerum Gottschl.

Hieracium dentatum Hoppe subsp. subnudum Nägeli \& Peter

Hieracium dentatum Hoppe subsp. subruncinatum Nägeli \& Peter

Hieracium dentatum Hoppe subsp. subvillosum Nägeli \& Peter

Hieracium dentatum Hoppe subsp. tigrinum Nägeli \& Peter

Hieracium dentatum Hoppe subsp. trefferianiforme Gottschl.

Hieracium dentatum Hoppe subsp. trefferianum (Nägeli \& Peter) Zahn

Hieracium dentatum Hoppe subsp. trentanum Nägeli \& Peter

Hieracium dentatum Hoppe subsp. tricephalum Nägeli \& Peter

Hieracium dentatum Hoppe subsp. turritiforme Nägeli \& Peter

Hieracium dentatum Hoppe subsp. vaudense (Arv.-Touv.) Zahn

Hieracium dentatum Hoppe subsp. villosiforme Nägeli \& Peter

E Hieracium dentatum Hoppe subsp. xanthostylophorum Furrer \& Zahn

E Hieracium dermophyllum Arv.-Touv. subsp. acutidens Gottschl.

Hieracium dermophyllum Arv.-Touv. subsp. dermophyllum

Hieracium dermophyllum Arv.-Touv. subsp. juraniforme (Zahn) Greuter

Hieracium dermophyllum Arv.-Touv. subsp. juranigenum (Zahn) Greuter

Hieracium dermophyllum Arv.-Touv. subsp. ortiganum (Zahn) Greuter

Hieracium diaphanoides Lindeb. subsp. cinerascentifolium Besse \& Zahn

Hieracium diaphanoides Lindeb. subsp. deductiforme Zahn

Hieracium diaphanoides Lindeb. subsp. diaphanoides

E Hieracium diaphanoides Lindeb. subsp. dimonense Gottschl.

E Hieracium diaphanoides Lindeb. subsp. forojuliense Gottschl.

Hieracium diaphanoides Lindeb. subsp. haslibergense Käser \& Zahn

Hieracium diaphanoides Lindeb. subsp. pseudumbrosum Zahn

Hieracium diaphanoides Lindeb. subsp. subelegans (Murr \& Zahn) Gottschl.

Hieracium diaphanoides Lindeb. subsp. vogesorum Zahn

Hieracium diaphanoides Lindeb. subsp. vorarlbergense (Murr \& Zahn) Zahn

Hieracium digenum Burn. \& Gremli

Hieracium dolichaetum Zahn

E Hieracium dollineri Sch.Bip. ex Neilr. subsp. addanum Zahn

E Hieracium dollineri Sch.Bip. ex Neilr. subsp. crinibasis Korb \& Zahn

E Hieracium dollineri Sch.Bip. ex Neilr. subsp. crinitellum Murr \& Zahn

Hieracium dollineri Sch.Bip. ex Neilr. subsp. dollineri

Hieracium dollineri Sch.Bip. ex Neilr. subsp. gadensiforme Murr \& Zahn

Hieracium dollineri Sch.Bip. ex Neilr. subsp. glaucogenes Murr \& Zahn

Hieracium dollineri Sch.Bip. ex Neilr. subsp. hyoseriophyllum Zahn

E Hieracium dollineri Sch.Bip. ex Neilr. subsp. pseudobaldense Murr \& Zahn

Hieracium dollineri Sch.Bip. ex Neilr. subsp. pseudofucatum Zahn

Hieracium dollineri Sch.Bip. ex Neilr. subsp. subcrinitellum Murr \& Zahn

Hieracium dollineri Sch.Bip. ex Neilr. subsp. tephromelanum Benz \& Zahn

Hieracium dollineri Sch.Bip. ex Neilr. subsp. tridentinum (Evers) Murr

Hieracium doronicifolium Arv.-Touv. subsp. doronicifolium

Hieracium doronicifolium Arv.-Touv. subsp. salevense (Rapin ex Fr.) Gottschl.

Hieracium dragicola (Nägeli \& Peter) Zahn subsp. carstiense (Nägeli \& Peter) Zahn

Hieracium dragicola (Nägeli \& Peter) Zahn subsp. dragicola

Hieracium dragicola (Nägeli \& Peter) Zahn subsp. pseudodragicola Zahn

Hieracium dragicola (Nägeli \& Peter) Zahn subsp. rossianum Zahn

Hieracium dunkelii Gottschl.

Hieracium duronense Gottschl.

ET Hieracium elbanum Belli ex Baroni

Hieracium erioleucum Zahn subsp. albatum (Nägeli \& Peter) Zahn

Hieracium erioleucum Zahn subsp. chionodes (Nägeli \& Peter) Zahn

Hieracium erioleucum Zahn subsp. erioleucum

Hieracium erioleucum Zahn subsp. eriovillosum (Nägeli \& Peter) Zahn

Hieracium erioleucum Zahn subsp. stenopodum Zahn

Hieracium erucophyllum Prain subsp. erucophyllum

Hieracium erucophyllum Prain subsp. prinzii (Käser ex Zahn) Greuter

E Hieracium erucopsis Gottschl.

E Hieracium exilicaule Gottschl.

Hieracium falcatum Arv.-Touv. subsp. falcatum

E Hieracium fastuosum Zahn

E Hieracium faucisjovis Gottschl.

Hieracium flagelliferum Ravaud

Hieracium froelichianum H.Buek subsp. beauverdianum (Besse \& Zahn) Gottschl. \& Greuter

E Hieracium froelichianum H.Buek subsp. cavicola Gottschl.

E Hieracium froelichianum H.Buek subsp. crepidens (Zahn) Gottschl. \& Greuter

E Hieracium froelichianum H.Buek subsp. epimediiforme (Benz \& Zahn) Gottschl. \& Greuter

Hieracium froelichianum H.Buek subsp. epimedium (Fr.) Gottschl. \& Greuter

Hieracium froelichianum $\mathrm{H}$.Buek subsp. exilentum (Arv.-Touv.) Gottschl. \& Greuter

Hieracium froelichianum $\mathrm{H}$.Buek subsp. froelichianum

Hieracium froelichianum H.Buek subsp. heterolobum (Zahn) Gottschl. \& Greuter 
Hieracium froelichianum H.Buek subsp. macilentum (Fr.) Gottschl. \& Greuter Hieracium froelichianum H.Buek subsp. punctiferum Gottschl.

Hieracium froelichianum H.Buek subsp. sparsipilum (Zahn) Gottschl. \& Greuter Hieracium froelichianum H.Buek subsp. subepimedium (Murr \& Zahn) Gottschl. \& Greuter Hieracium froelichianum $\mathrm{H}$.Buek subsp. subvulsum (Zahn) Gottschl. \& Greuter Hieracium froelichianum H.Buek subsp. wimmerioides (Zahn) Gottschl. \& Greuter

E Hieracium fulcratum Arv.-Touv. subsp. lillense (Zahn) Zahn

E Hieracium galeroides Gottschl. subsp. aculeatisquamum Gottschl.

E Hieracium galeroides Gottschl. subsp. galeroides

E Hieracium geilingeri Zahn subsp. brembanae Wilczek \& Zahn

Hieracium geilingeri Zahn subsp. geilingeri

Hieracium glabratum Hoppe ex Willd. subsp. doratolepium Nägeli \& Peter

Hieracium glabratum Hoppe ex Willd. subsp. glabratum

Hieracium glabratum Hoppe ex Willd. subsp. gymnophyllum Nägeli \& Peter

Hieracium glabratum Hoppe ex Willd. subsp. leontinum Nägeli \& Peter

Hieracium glabratum Hoppe ex Willd. subsp. niphoneuron Nägeli \& Peter

Hieracium glabratum Hoppe ex Willd. subsp. nudum A.Kern. ex Nägeli \& Peter

Hieracium glabratum Hoppe ex Willd. subsp. pseudoflexuosum Nägeli \& Peter

Hieracium glabratum Hoppe ex Willd. subsp. senecioides (Arv.-Touv. \& Gaut.) Zahn

Hieracium glanduliferum Hoppe subsp. fuliginatum Huter \& Gander ex Nägeli \& Peter

Hieracium glanduliferum Hoppe subsp. glanduliferum

Hieracium glanduliferum Hoppe subsp. hololeptum Nägeli \& Peter

Hieracium glanduliferum Hoppe subsp. leucopsis Arv.-Touv. ex Nägeli \& Peter

Hieracium glanduliferum Hoppe subsp. piliferum Hoppe ex Nägeli \& Peter

Hieracium glanduliferum Hoppe subsp. subnivale (Gren. \& Godr.) Nägeli \& Peter

E Hieracium glanduliferum Hoppe subsp. subnivaliforme Zahn

E Hieracium glaucinum Jord. subsp. acrocampylum (Zahn) Greuter

Hieracium glaucinum Jord. subsp. arnicoides (Gren. \& Godr.) O.Bolòs \& Vigo

Hieracium glaucinum Jord. subsp. bounophilum (Jord. ex Boreau) O.Bolòs \& Vigo

Hieracium glaucinum Jord. subsp. cinerascens (Jord.) Breistr. \& Litard.

E Hieracium glaucinum Jord. subsp. cispadanum (Fen. \& Zahn) Greuter

Hieracium glaucinum Jord. subsp. conjugatum (Jord. ex Boreau) O.Bolòs \& Vigo

Hieracium glaucinum Jord. subsp. fallens (Jord. ex Boreau) Gottschl.

Hieracium glaucinum Jord. subsp. fragile (Jord.) O.Bolòs \& Vigo

Hieracium glaucinum Jord. subsp. fraternum (Sudre) Soó

Hieracium glaucinum Jord. subsp. glaucinum

Hieracium glaucinum Jord. subsp. heteroschistum (Zahn) Soó

Hieracium glaucinum Jord. subsp. jaubertianum (Timb.-Lagr. \& Loret) O.Bolòs \& Vigo

Hieracium glaucinum Jord. subsp. oigocladum (Jord. ex Boreau) Soó

Hieracium glaucinum Jord. subsp. ottanense (Zahn) Greuter

Hieracium glaucinum Jord. subsp. ovalifolium (Jord.) Soó

Hieracium glaucinum Jord. subsp. pallidifrons (Sudre) O.Bolòs \& Vigo

Hieracium glaucinum Jord. subsp. pallidulum (Jord. ex Boreau) Soó

Hieracium glaucinum Jord. subsp. petiolare (Jord.) Greuter

Hieracium glaucinum Jord. subsp. pinicola (Sudre) Gottschl.

Hieracium glaucinum Jord. subsp. prasinifolium (Jord. ex Boreau) O.Bolòs \& Vigo

Hieracium glaucinum Jord. subsp. prasiophaeum (Arv.-Touv. \& Gaut.) Gottschl.

E Hieracium glaucinum Jord. subsp. pseudobasalticum Gottschl.

Hieracium glaucinum Jord. subsp. pseudobounophilum (Zahn) Gottschl.

Hieracium glaucinum Jord. subsp. pseudocyaneum (Zahn) O.Bolòs \& Vigo

Hieracium glaucinum Jord. subsp. pulverosum (Zahn) Greuter

Hieracium glaucinum Jord. subsp. recensitum (Jord. ex Boreau) Gottschl.

Hieracium glaucinum Jord. subsp. similatum (Jord. ex Boreau) Gottschl.

Hieracium glaucinum Jord. subsp. subfraternum (Zahn) Greuter

Hieracium glaucinum Jord. subsp. subpubescens (Zahn) Greuter

E Hieracium glaucinum Jord. subsp. tintiae Gottschl.

Hieracium glaucinum Jord. subsp. valdevestitum (Besse \& Zahn) Greuter

E Hieracium glaucinum Jord. subsp. valpergae Gottschl.

Hieracium glaucopsis Gren. \& Godr. subsp. glaucopsis

Hieracium glaucum All. subsp. amaurodes Nägeli \& Peter

Hieracium glaucum All. subsp. chiamuerae Nägeli \& Peter

Hieracium glaucum All. subsp. chrysostylum Nägeli \& Peter

Hieracium glaucum All. subsp. glaucum

Hieracium glaucum All. subsp. gymnolepium Nägeli \& Peter

Hieracium glaucum All. subsp. isaricum (Nägeli ex J.Hofm.) Prantl

Hieracium glaucum All. subsp. limonense (Burnat \& Gremli) Zahn

Hieracium glaucum All. subsp. nipholepium Nägeli \& Peter

E Hieracium glaucum All. subsp. serenaiae Gottschl.

Hieracium glaucum All. subsp. stenobracteum Nägeli \& Peter

E Hieracium glaucum All. subsp. subglaucum (Burnat \& Gremli) Zahn

E Hieracium glaucum All. subsp. subporrifolium Nägeli \& Peter

Hieracium glaucum All. subsp. subturbinatum Nägeli \& Peter

Hieracium glaucum All. subsp. tenerum Nägeli \& Peter

Hieracium glaucum All. subsp. tephrolepium Nägeli \& Peter

Hieracium glaucum All. subsp. turbinatum Nägeli \& Peter

Hieracium glaucum All. subsp. willdenowii (Monnier) Nägeli \& Peter

Hieracium gombense Lagger ex Christener subsp. praemontanum (Benz \& Zahn) Zahn

Hieracium gombense Lagger ex Christener subsp. weitfeldense (Murr) Zahn 
E Hieracium grovesianum Arv.-Touv. ex Belli subsp. grovesianum

Hieracium grovesianum Arv.-Touv. ex Belli subsp. Iuteobarbatum Gottschl.

Hieracium grovesianum Arv.-Touv. ex Belli subsp. nigrotectorum Gottschl.

Hieracium grovesianum Arv.-Touv. ex Belli subsp. peracutum Gottschl.

Hieracium grovesianum Arv.-Touv. ex Belli subsp. pseuditalicum (Zahn) Gottschl.

Hieracium grovesianum Arv.-Touv. ex Belli subsp. rigoanum (Zahn) Zahn

Hieracium grovesianum Arv.-Touv. ex Belli subsp. rivasolanum Zahn

Hieracium grovesianum Arv.-Touv. ex Belli subsp. serratifolioides Gottschl.

Hieracium grovesianum Arv.-Touv. ex Belli subsp. stenofolium Gottschl.

Hieracium grovesianum Arv.-Touv. ex Belli subsp. varellanum (Zahn) Zahn

Hieracium hayekii Murr

Hieracium hispidulum Arv.-Touv. subsp. hispidulum

Hieracium huetianum Arv.-Touv.

Hieracium humile Jacq. subsp. brachycaule Vuk. ex Zahn

Hieracium humile Jacq. subsp. humile

Hieracium humile Jacq. subsp. Iacerum (Reut. ex Fr.) Zahn

Hieracium humile Jacq. subsp. pseudocottetii Zahn

Hieracium hypochoeroides S.Gibson subsp. adesiforme (Zahn) Greuter

Hieracium hypochoeroides S.Gibson subsp. adesum (Bernoulli \& Zahn) Greuter

Hieracium hypochoeroides S.Gibson subsp. bifidopsis (Zahn) Greuter

Hieracium hypochoeroides S.Gibson subsp. bridelianum (Zahn) Greuter

E Hieracium hypochoeroides S.Gibson subsp. cilentanum Di Grist., Gottschl. \& Raimondo

Hieracium hypochoeroides S.Gibson subsp. cinerascentiforme (Zahn) Greuter

Hieracium hypochoeroides S.Gibson subsp. dolichellum (Arv.-Touv. \& Gaut.) Greuter

Hieracium hypochoeroides S.Gibson subsp. glaucibifidum (Arv.-Touv. \& Gaut.) Greuter

Hieracium hypochoeroides S.Gibson subsp. grandisaxense Gottschl.

Hieracium hypochoeroides S.Gibson subsp. hemibifidum (Zahn) Greuter

Hieracium hypochoeroides S.Gibson subsp. lineatiforme (Zahn) Greuter

Hieracium hypochoeroides S.Gibson subsp. lineatoides (Zahn) Greuter

Hieracium hypochoeroides S.Gibson subsp. lithophilum (Arv.-Touv.) Greuter

E Hieracium hypochoeroides S.Gibson subsp. Iucanicum (Arv.-Touv.) Di Grist., Gottschl. \& Raimondo

E Hieracium hypochoeroides S.Gibson subsp. montis-scuderii Di Grist., Gottschl., Galesi, Raimondo \& Cristaudo

Hieracium hypochoeroides S.Gibson subsp. nervulosum (Arv.-Touv. \& Gaut.) Greuter

E Hieracium hypochoeroides S.Gibson subsp. pallidopsis Gottschl.

E Hieracium hypochoeroides S.Gibson subsp. peracutisquamum Di Grist., Gottschl. \& Raimondo

E Hieracium hypochoeroides S.Gibson subsp. pinnatiscissum (Zahn) Greuter

E Hieracium hypochoeroides S.Gibson subsp. potamogetifolium Gottschl.

Hieracium hypochoeroides S.Gibson subsp. prasinophyton (Zahn) Greuter

Hieracium hypochoeroides S.Gibson subsp. pseudograniticum (Besse \& Zahn) Greuter

E Hieracium hypochoeroides S.Gibson subsp. serinense (Zahn) Greuter

Hieracium hypochoeroides S.Gibson subsp. subcanens (Arv.-Touv. \& Gaut.) Greuter

Hieracium hypochoeroides S.Gibson subsp. subcinereum (Arv.-Touv. \& Gaut.) Greuter

E Hieracium hypochoeroides S.Gibson subsp. sublanigerum (Arv.-Touv. ex Belli) Gottschl. \& Wagens.

Hieracium hypochoeroides S.Gibson subsp. sublineolatum (Zahn) Greuter

E Hieracium hypochoeroides S.Gibson subsp. supramontanum (Arrigoni) Greuter

Hieracium hypochoeroides S.Gibson subsp. wiesbaurianum (R.Uechtr.) Greuter

E Hieracium illyricopsis Gottschl.

E Hieracium insubricum Gottschl.

Hieracium intertextum Arv.-Touv.

Hieracium inuloides Tausch subsp. Ianceolatifolium (Zahn) Zahn

Hieracium inuloides Tausch subsp. poellianum Zahn

Hieracium inuloides Tausch subsp. subconicum Zahn

Hieracium inuloides Tausch subsp. subtridentatum (Zahn) Zahn

Hieracium inuloides Tausch subsp. subvirens (Arv.-Touv.) Greuter

E Hieracium inuloides Tausch subsp. subvirentiforme Zahn

Hieracium inuloides Tausch subsp. tridentatifolium (Zahn) Zahn

Hieracium inuloides Tausch subsp. vetteri Zahn

Hieracium isatidifolium Arv.-Touv. subsp. isatidifolium

Hieracium isolanum Zahn

Hieracium jordanii Arv.-Touv. subsp. jordanii

Hieracium jordanii Arv.-Touv. subsp. lacistum (Nägeli \& Peter) Zahn

Hieracium jordanii Arv.-Touv. subsp. pseudotomentosum (Nägeli \& Peter) Zahn

Hieracium jordanii Arv.-Touv. subsp. yvesianum (Arv.-Touv.) Zahn

E Hieracium juengeri Gottschl.

Hieracium juranum Rapin subsp. juranum

Hieracium jurassicum Griseb. subsp. adenocalathium (Zahn) Greuter

Hieracium jurassicum Griseb. subsp. aligerum (Zahn) Greuter

Hieracium jurassicum Griseb. subsp. amplexifolium (Arv.-Touv.) Greuter

Hieracium jurassicum Griseb. subsp. cichoriaceum (Arv.-Touv.) Gottschl.

Hieracium jurassicum Griseb. subsp. constrictiforme (Zahn) Greuter

Hieracium jurassicum Griseb. subsp. decrescentifolium (Murr \& Zahn) Greuter

E Hieracium jurassicum Griseb. subsp. decrescentifrons (Zahn) Greuter

Hieracium jurassicum Griseb. subsp. elegantissimum (Zahn) Gottschl.

Hieracium jurassicum Griseb. subsp. fontanalbae (C.Bicknell \& Zahn) Greuter

Hieracium jurassicum Griseb. subsp. hemiplecoides (Arv.-Touv. \& Gaut.) Greuter

Hieracium jurassicum Griseb. subsp. hemiplecum (Arv.-Touv.) Greuter

Hieracium jurassicum Griseb. subsp. jaceoides (Arv.-Touv.) Gottschl.

Hieracium jurassicum Griseb. subsp. jurassicum 
Hieracium jurassicum Griseb. subsp. Iancifrons (Zahn) Greuter Hieracium jurassicum Griseb. subsp. prenanthopsis (Murr \& Zahn) Gottschl. Hieracium jurassicum Griseb. subsp. pseudohemiplecum (Zahn) Gottschl. Hieracium jurassicum Griseb. subsp. pseudojuranum (Arv.-Touv.) Greuter Hieracium jurassicum Griseb. subsp. stenoplecopsis (Zahn) Greuter Hieracium jurassicum Griseb. subsp. subperfoliatum (Arv.-Touv.) Greuter Hieracium jurassicum Griseb. subsp. translucens (Arv.-Touv.) Greuter Hieracium kalsianum Huter subsp. kalsianum Hieracium kalsianum Huter subsp. ochroleucopsis Zahn Hieracium kofelicum Gottschl.

Hieracium lachenalii Suter subsp. acidodontophyllum (Zahn) Zahn

Hieracium lachenalii Suter subsp. acuminatum (Jord.) Zahn

Hieracium lachenalii Suter subsp. acutatifrons (Zahn) Zahn Hieracium lachenalii Suter subsp. alpestre (R.Uechtr.) Greuter Hieracium lachenalii Suter subsp. amaurochlorum (Zahn) Zahn Hieracium lachenalii Suter subsp. anfractum (Fr.) Zahn Hieracium lachenalii Suter subsp. argillaceum (Jord.) Zahn Hieracium lachenalii Suter subsp. aspernatum (Jord. ex Boreau) Zahn Hieracium lachenalii Suter subsp. asyngamicum (Borbás) Zahn Hieracium lachenalii Suter subsp. aurulentum (Jord. ex Boreau) Zahn Hieracium Iachenalii Suter subsp. ausserdorferi (Hausm. ex Zahn) Zahn Hieracium lachenalii Suter subsp. aviicola (Jord. ex Boreau) Zahn Hieracium lachenalii Suter subsp. bathylepium (Dahlst.) Zahn Hieracium lachenalii Suter subsp. brevidentatum (Jord. ex Boreau) Zahn Hieracium lachenalii Suter subsp. chlorophyllum (Jord. ex Boreau) Zahn

E Hieracium lachenalii Suter subsp. chrysoglossoides (Zahn) Zahn Hieracium lachenalii Suter subsp. euchlorum (Murr \& Zahn) Zahn Hieracium lachenalii Suter subsp. fastigiatum (Fr.) Zahn Hieracium lachenalii Suter subsp. festinum (Jord. ex Boreau) Zahn Hieracium lachenalii Suter subsp. frondosiforme (Zahn) Zahn Hieracium lachenalii Suter subsp. garckeanum (Asch.) Zahn Hieracium lachenalii Suter subsp. haematochromum (Zahn) Zahn

Hieracium lachenalii Suter subsp. interaquense (Zahn) Zahn Hieracium lachenalii Suter subsp. irriguum (Fr.) Zahn Hieracium lachenalii Suter subsp. Iachenalii Hieracium lachenalii Suter subsp. lepiduloides Zahn Hieracium Iachenalii Suter subsp. Iupimontis (Zahn) Zahn Hieracium lachenalii Suter subsp. mutiense (Zahn) Zahn Hieracium lachenalii Suter subsp. obscuriceps (Dahlst.) Greuter Hieracium Iachenalii Suter subsp. paucifoliatum (Jord. ex Boreau) Zahn Hieracium lachenalii Suter subsp. perscissum (Jord. ex Boreau) Zahn Hieracium lachenalii Suter subsp. pinnatifidum (Dahlst.) Zahn Hieracium lachenalii Suter subsp. purpureoviolascens (Zahn) Zahn Hieracium lachenalii Suter subsp. scanicum (Dahlst.) Zahn Hieracium lachenalii Suter subsp. subampliatum (Dahlst.) Zahn Hieracium lachenalii Suter subsp. subfastigiatum (Zahn) Zahn Hieracium lachenalii Suter subsp. subirriguifrons (Zahn) Zahn Hieracium lachenalii Suter subsp. subirriguum (Dahlst.) Zahn Hieracium lachenalii Suter subsp. sublepiduliforme Harz \& Zahn Hieracium lachenalii Suter subsp. subpunctillatiforme (Zahn) Zahn Hieracium Iachenalii Suter subsp. subviriduliceps (Zahn) Zahn Hieracium lachenalii Suter subsp. tortifolium (Jord. ex Boreau) Zahn

E Hieracium laevigatum Willd. subsp. albaredanum Zahn Hieracium laevigatum Willd. subsp. amaurolepis Murr \& Zahn Hieracium laevigatum Willd. subsp. gothiciforme (Dahlst.) Zahn Hieracium laevigatum Willd. subsp. grandidens Zahn Hieracium laevigatum Willd. subsp. gsiesense Zahn Hieracium laevigatum Willd. subsp. knafii (Celak.) Zahn Hieracium laevigatum Willd. subsp. Iaevigans Zahn Hieracium laevigatum Willd. subsp. laevigatum Hieracium laevigatum Willd. subsp. lineatum (Dahlst.) Zahn Hieracium laevigatum Willd. subsp. magistri (Godr.) Zahn Hieracium laevigatum Willd. subsp. nivale (Froel.) Zahn Hieracium laevigatum Willd. subsp. pictaviense (Sauzé \& Maillard) Zahn Hieracium laevigatum Willd. subsp. pizzulicum Gottschl. Hieracium laevigatum Willd. subsp. pseudogothicum (Arv.-Touv.) Zahn Hieracium laevigatum Willd. subsp. retardatum Zahn Hieracium laevigatum Willd. subsp. rigidum (Hartm.) Zahn Hieracium laevigatum Willd. subsp. stenogonium Zahn Hieracium laevigatum Willd. subsp. trichocalyx Gottschl. Hieracium laevigatum Willd. subsp. tridentatum (Fr.) Celak. Hieracium lanseanum Arv.-Touv. subsp. Ianseanum Hieracium lawsonii Vill. subsp. lawsonii 
Hieracium leiocephalum Bartl. ex Griseb. subsp. melanocalyx (Nägeli \& Peter) Zahn

Hieracium leiocephalum Bartl. ex Griseb. subsp. subumbellatum (Nägeli \& Peter) Gottschl.

Hieracium leiocephalum Bartl. ex Griseb. subsp. timaviense (Nägeli \& Peter) Zahn

Hieracium leiophaeum Arv.-Touv.

Hieracium leiopogon Gren. ex Verl. subsp. hyposericum Zahn

Hieracium leiopogon Gren. ex Verl. subsp. iolai (Arrigoni) Greuter

Hieracium leucophaeum Gren. \& Godr. subsp. asterinum (Arv.-Touv. \& Briq.) Zahn

Hieracium leucophaeum Gren. \& Godr. subsp. diabolinum (Nägeli \& Peter) Zahn

Hieracium leucophaeum Gren. \& Godr. subsp. gremlii (Arv.-Touv.) Zahn

Hieracium leucophaeum Gren. \& Godr. subsp. leucophaeum

Hieracium levicaule Jord. subsp. acroleucum (Dahlst.) Zahn

Hieracium levicaule Jord. subsp. ausugum (Murr \& Zahn) Zahn

Hieracium levicaule Jord. subsp. calcigenum (Rehm.) Zahn

Hieracium levicaule Jord. subsp. campylodon (Zahn) Greuter

Hieracium levicaule Jord. subsp. chloroviolaceum F.Feurstein \& Zahn

Hieracium levicaule Jord. subsp. davallianum (Zahn) Zahn

Hieracium levicaule Jord. subsp. eurobremium Zahn

Hieracium levicaule Jord. subsp. euroum (Murr \& Zahn) Zahn

Hieracium levicaule Jord. subsp. fissicladum (Zahn) Zahn

Hieracium levicaule Jord. subsp. lepidulum (Dahlst.) Greuter

Hieracium levicaule Jord. subsp. Ionchodes (Murr \& Zahn) Zahn

Hieracium levicaule Jord. subsp. psammogeton (Zahn) Zahn

Hieracium levicaule Jord. subsp. pseudosenile (Zahn) Zahn

Hieracium levicaule Jord. subsp. siderense (Zahn) Zahn

Hieracium levicaule Jord. subsp. triviale (Norrl.) Zahn

Hieracium levicaule Jord. subsp. vitulimontis Zahn

Hieracium levicaule Jord. subsp. vulgatiflorum (Zahn) Zahn

Hieracium liptoviense Borbás

Hieracium longifolium Schleich. ex Hornem. subsp. Iongifolium

Hieracium longifolium Schleich. ex Hornem. subsp. neocerinthoides (Arv.-Touv. \& Briq.) Greuter

E Hieracium lucidum Guss.

Hieracium lychnioides Arv.-Touv.

Hieracium lycopifolium Froel. subsp. bifrons (Arv.-Touv.) Zahn

Hieracium lycopifolium Froel. subsp. lycopifolioides Zahn

Hieracium lycopifolium Froel. subsp. Iycopifolium

E Hieracium lycopifolium Froel. subsp. ocreanum Gottschl.

Hieracium lycopifolium Froel. subsp. pseudeminens Zahn

Hieracium lycopifolium Froel. subsp. sabaudifolium Zahn

Hieracium lycopifolium Froel. subsp. trichoprenanthes (Zahn) Zahn

Hieracium lycopifolium Froel. subsp. vallisiacum (Fr.) Zahn

E Hieracium macrogrovesianum Gottschl. subsp. colfioritense Gottschl.

E Hieracium macrogrovesianum Gottschl. subsp. macrogrovesianum

Hieracium maculatum Schrank subsp. arrectariicaule (Sudre) Greuter

Hieracium maculatum Schrank subsp. astictum F.Feurstein \& Zahn

Hieracium maculatum Schrank subsp. commixtum (Jord.) Zahn

Hieracium maculatum Schrank subsp. cruentum (Jord.) Zahn

Hieracium maculatum Schrank subsp. divisum (Jord.) Zahn

Hieracium maculatum Schrank subsp. maculatum

E Hieracium marchesonii Gottschl.

E Hieracium marsorum Gottschl.

Hieracium mattiroloanum Arv.-Touv. \& Belli subsp. farinulentiforme (Zahn) Greuter

Hieracium mattiroloanum Arv.-Touv. \& Belli subsp. martellianum (Zahn) Greuter

E Hieracium medense Gottschl. \& Dunkel

Hieracium melanops Arv.-Touv. subsp. mairanum (Belli) Greuter

Hieracium melanops Arv.-Touv. subsp. melanops

Hieracium melanops Arv.-Touv. subsp. subadusticeps (Zahn) Gretuer

Hieracium misaucinum Nägeli \& Peter subsp. misaucinum

Hieracium misaucinum Nägeli \& Peter subsp. prionotum (Arv.-Touv.) Zahn

Hieracium mollitum Arv.-Touv. subsp. mollitum

Hieracium monnieri Arv.-Touv. subsp. goderiense (Zahn) Zahn

Hieracium monregalense Burnat \& Gremli subsp. gibellianum (Arv.-Touv. \& Belli) Zahn

Hieracium monregalense Burnat \& Gremli subsp. Ianosum (Nägeli \& Peter) Zahn

Hieracium monregalense Burnat \& Gremli subsp. monregalense

Hieracium monregalense Burnat \& Gremli subsp. villiferum (Nägeli \& Peter) Zahn

Hieracium montis-florum Gottschl. subsp. montis-florum

Hieracium montis-florum Gottschl. subsp. soldanoi Gottschl.

Hieracium montis-porrarae Gottschl.

Hieracium murorum L. subsp. adenobifidum Wilczek \& Zahn

Hieracium murorum L. subsp. amaurocymum (Touton \& Zahn ex Dalla Torre \& Sarnth.) Greuter

E Hieracium murorum L. subsp. anisobasis Gottschl.

Hieracium murorum L. subsp. aspreticola (Jord. ex Boreau) Zahn

Hieracium murorum L. subsp. atropaniculatum (Zahn) Zahn

E Hieracium murorum L. subsp. atrovirens (Froel.) Raimondo \& Di Grist.

Hieracium murorum L. subsp. basalticiforme Korb \& Zahn

Hieracium murorum L. subsp. bifidiforme (Zahn) Zahn

Hieracium murorum L. subsp. boetzkesii Zahn

Hieracium murorum L. subsp. brachylobosum J.Vetter \& Zahn

Hieracium murorum L. subsp. calvifrons Zahn 
Hieracium murorum L. subsp. cardiophyllum (Sudre) Zahn Hieracium murorum L. subsp. circumstellatum (Zahn) Zahn Hieracium murorum L. subsp. cirritoides (Zahn) Zahn

Hieracium murorum L. subsp. cirritoidiforme Zahn

Hieracium murorum L. subsp. crepidanthes Zahn

Hieracium murorum L. subsp. crepidiflorum (Polák) Zahn

Hieracium murorum $L$. subsp. denticulatifrons J.Vetter \& Zahn

Hieracium murorum L. subsp. erucifolium (Arv.-Touv.) Zahn

Hieracium murorum L. subsp. exotericum (Jord. ex Boreau) Sudre

Hieracium murorum L. subsp. flavescens (Evers) Zahn

Hieracium murorum L. subsp. fritschii (Pernh.) Zahn

Hieracium murorum L. subsp. gentile (Jord. ex Boreau) Sudre

E Hieracium murorum L. subsp. gigantolobum Zahn

Hieracium murorum L. subsp. glandellatum Zahn

Hieracium murorum L. subsp. graminicolor (Zahn) Zahn

E Hieracium murorum L. subsp. graminochlorum Fen. \& Zahn

E Hieracium murorum L. subsp. grypharioides Zahn

Hieracium murorum L. subsp. hemigrypotes Zahn

E Hieracium murorum L. subsp. heteroserratum Gottschl.

E Hieracium murorum L. subsp. infralobatum Zahn

Hieracium murorum L. subsp. infrasericatum Murr \& Zahn

Hieracium murorum L. subsp. integratum (Dahlst.) Zahn

Hieracium murorum L. subsp. kassanum Zahn

Hieracium murorum L. subsp. Iongilacerum Murr \& Zahn

Hieracium murorum L. subsp. Iorynsicum Krafft \& Zahn

E Hieracium murorum L. subsp. marmarolense Pamp. \& Zahn

E Hieracium murorum L. subsp. megaleiobium Pamp. \& Zahn

E Hieracium murorum L. subsp. melanopsiforme Zahn

Hieracium murorum L. subsp. melanosphaeroides Romieux \& Zahn

Hieracium murorum L. subsp. microspilon (Sudre) Zahn

Hieracium murorum L. subsp. mundellum Zahn

Hieracium murorum L. subsp. nemorense (Jord.) Zahn

E Hieracium murorum L. subsp. nivimarginatum Gottschl.

Hieracium murorum L. subsp. oblongum (Jord.) Zahn

Hieracium murorum L. subsp. odontobium Zahn

Hieracium murorum L. subsp. oxyodontopsis (Touton \& Zahn) Zahn

Hieracium murorum L. subsp. persinuatum Zahn

Hieracium murorum L. subsp. perviride Zahn

Hieracium murorum L. subsp. pictoprasinum Fen. \& Zahn

Hieracium murorum L. subsp. pleiotrichum (Zahn) Zahn

Hieracium murorum L. subsp. polygonium Zahn

Hieracium murorum L. subsp. psammogenoides Zahn

Hieracium murorum L. subsp. pseudobifidiforme Pamp. \& Zahn

Hieracium murorum L. subsp. pseudodiaphanoides Gerstl. \& Zahn

E Hieracium murorum L. subsp. pseudofritschii Zahn

Hieracium murorum L. subsp. pseudomedianum Besse \& Zahn

Hieracium murorum L. subsp. pseudomerianum P.Rossi \& Zahn

Hieracium murorum L. subsp. pseudosilvularum Zahn

E Hieracium murorum L. subsp. pseudosubulatidens Pamp. \& Zahn

Hieracium murorum L. subsp. ramosiforme (Zahn) Zahn

Hieracium murorum L. subsp. samnaunicum (Zahn) Gottschl.

E Hieracium murorum L. subsp. semicontractum P.Rossi \& Zahn

Hieracium murorum L. subsp. semisilvaticiforme Zahn

Hieracium murorum L. subsp. semisilvaticum (Zahn) Zahn

Hieracium murorum L. subsp. semisilvularum Harz \& Zahn

Hieracium murorum L. subsp. serratifolium (Jord. ex Boreau) Zahn

Hieracium murorum L. subsp. sparsum (Jord. ex Sudre) Zahn

Hieracium murorum L. subsp. subbifidiforme Zahn

E Hieracium murorum L. subsp. subcoriifolium Zahn

Hieracium murorum L. subsp. subcrassum (Dahlst.) Zahn

Hieracium murorum L. subsp. subditivum (Zahn) Zahn

E Hieracium murorum L. subsp. subinfrasericatum P.Rossi \& Zahn

E Hieracium murorum $L$. subsp. subintegerrimum Gottschl.

Hieracium murorum L. subsp. subnemorense (Zahn) Zahn

Hieracium murorum L. subsp. subsemisilvularum (Zahn) Zahn

Hieracium murorum L. subsp. sylvivagum (Jord. ex Boreau) Zahn

Hieracium murorum L. subsp. sylvularum (Jord. ex Boreau) Zahn

Hieracium murorum L. subsp. tofanense Pamp. \& Zahn

Hieracium murorum L. subsp. torticeps (Dahlst.) Zahn

E Hieracium murorum L. subsp. triangulilacerum P.Rossi \& Zahn

Hieracium murorum L. subsp. valdefastigiatum (Zahn ex Dalla Torre \& Sarnth.) Zahn

Hieracium murorum L. subsp. variilobum Zahn

Hieracium murorum L. subsp. wulfenii Zahn

Hieracium naegelianum Pančić subsp. andreae (Degen \& Zahn) Zahn

E Hieracium nematopodum (Zahn) P.D.Sell \& C.West

Hieracium neomalyi Zahn

E Hieracium neoplatyphyllum Gottschl. subsp. izzense Gottschl. 
Hieracium neoplatyphyllum Gottschl. subsp. malacofloccosum Gottschl.

Hieracium neoplatyphyllum Gottschl. subsp. neoplatyphyllum

E Hieracium neoplatyphyllum Gottschl. subsp. trimontanum Gottschl.

E Hieracium neronense Gottschl.

E Hieracium neyranum Arv.-Touv. subsp. glaucocephalum (Nägeli \& Peter) Greuter

E Hieracium neyranum Arv.-Touv. subsp. grausonicum Besse \& Zahn

Hieracium neyranum Arv.-Touv. subsp. neyranum

Hieracium nigrescens Willd. subsp. cochleare (Huter) Zahn

Hieracium nigrescens Willd. subsp. leontodontoides (Arv.-Touv. \& Briq.) Zahn

Hieracium nigrescens Willd. subsp. nigrescentiforme Zahn

Hieracium nigrescens Willd. subsp. pseudohalleri Zahn

Hieracium nigrescens Willd. subsp. pseudorhaeticum Zahn

Hieracium nigrescens Willd. subsp. rhaeticum (Fr.) Zahn

Hieracium nigrescens Willd. subsp. subneglectum (Zahn) Zahn

Hieracium nigrescens Willd. subsp. subpumilum (Zahn) Zahn

Hieracium niveobarbatum Arv.-Touv. ex Gottschl.

E Hieracium nubitangens Gottschl.

Hieracium obscuratum Murr subsp. nigratum (Murr \& Zahn) Greuter

Hieracium obscuratum Murr subsp. obscuratum

E Hieracium obscuratum Murr subsp. sampeyrense Gottschl.

E Hieracium obscuratum Murr subsp. violae (Fen. \& Zahn) Greuter

Hieracium oligodon Nägeli \& Peter subsp. oligodon

Hieracium onosmoides Fr. subsp. onosmoides

Hieracium onosmoides Fr. subsp. subrude (Arv.-Touv.) Zahn

Hieracium oreiocephalum Zahn subsp. oreiocephalum

E Hieracium orodoxum Gottschl. subsp. orodoxum

E Hieracium orodoxum Gottschl. subsp. pseudonaegelianum Gottschl.

E Hieracium orsierae Gottschl.

Hieracium oxyodon Fr. subsp. ganderi (Zahn) Zahn

Hieracium oxyodon Fr. subsp. incisiforme (Murr) Zahn

Hieracium oxyodon Fr. subsp. inclinatum (Arv.-Touv.) Zahn

E Hieracium oxyodon Fr. subsp. leucochaetopodum Fen. \& Zahn

Hieracium oxyodon Fr. subsp. leucopodum (Zahn) Zahn

E Hieracium oxyodon Fr. subsp. Ionganum (Arv.-Touv.) Zahn

Hieracium oxyodon Fr. subsp. muretii (Gremli) Zahn

Hieracium oxyodon Fr. subsp. oxyodon

Hieracium oxyodon Fr. subsp. pseudoscabrellum Zahn

Hieracium oxyodon Fr. subsp. subcanescens (Murr) Zahn

Hieracium oxyodon $\mathrm{Fr}$. subsp. tomense (Zahn) Zahn

E Hieracium pallescens Waldst. \& Kit. subsp. ciliatifolium (Zahn) Gottschl.

E Hieracium pallescens Waldst. \& Kit. subsp. gelmianum (Sarnth.) Gottschl. Hieracium pallescens Waldst. \& Kit. subsp. hittense (Murr) Greuter Hieracium pallescens Waldst. \& Kit. subsp. humiliforme (Zahn) Gottschl. Hieracium pallescens Waldst. \& Kit. subsp. incisum (Hoppe) Greuter Hieracium pallescens Waldst. \& Kit. subsp. macranthoides (Zahn) Gottschl. Hieracium pallescens Waldst. \& Kit. subsp. muroriforme (Zahn) Gottschl.

Hieracium pallescens Waldst. \& Kit. subsp. murrianum (Arv.-Touv. ex Murr) Gottschl. Hieracium pallescens Waldst. \& Kit. subsp. naevulatum (Zahn) Greuter

Hieracium pallescens Waldst. \& Kit. subsp. oligomeres (Murr \& Zahn) Greuter Hieracium pallescens Waldst. \& Kit. subsp. ovale (Zahn) Gottschl. Hieracium pallescens Waldst. \& Kit. subsp. oxyodontiforme (Zahn) Greuter Hieracium pallescens Waldst. \& Kit. subsp. paicheanum (Briq.) Greuter Hieracium pallescens Waldst. \& Kit. subsp. pallescens

Hieracium pallescens Waldst. \& Kit. subsp. pseudotrachselianum (Zahn) Gottschl. Hieracium pallescens Waldst. \& Kit. subsp. rhoeadiolium (A.Kern. ex Willk.) Gottschl. Hieracium pallescens Waldst. \& Kit. subsp. subpatulum (Zahn ex Benz) Greuter Hieracium pallescens Waldst. \& Kit. subsp. tephrochlorum (Zahn) Gottschl. Hieracium pallescens Waldst. \& Kit. subsp. trachselianoides (Zahn) Greuter Hieracium pallidum Biv. subsp. aetnense Gottschl., Raimondo \& Di Grist. Hieracium pallidum Biv. subsp. Ianudae (Gottschl.) Gottschl., Raimondo \& Di Grist. Hieracium pallidum Biv. subsp. pallidum Hieracium pamphili Arv.-Touv.

Hieracium pedemontanum Burnat \& Gremli

E Hieracium pellitum $\mathrm{Fr}$. subsp. leuconeuron Zahn Hieracium pellitum Fr. subsp. oligocephalum (Arv.-Touv.) Zahn Hieracium pellitum $\mathrm{Fr}$. subsp. pellitum

Hieracium pellitum $\mathrm{Fr}$. subsp. pictifolium Zahn

E Hieracium pellitum Fr. subsp. praetenue (Besse \& Zahn) Zahn

Hieracium pellitum Fr. subsp. prasinellum (C.Bicknell \& Zahn) Zahn

Hieracium pellitum Fr. subsp. pseudolanatum (Arv.-Touv.) Zahn

Hieracium pellitum Fr. subsp. quercifolioides Zahn

Hieracium permaculatum Gottschl. subsp. cacuminicola Gottschl.

Hieracium permaculatum Gottschl. subsp. permaculatum

Hieracium picenorum Gottschl. subsp. falsobifidum Gottschl.

Hieracium picenorum Gottschl. subsp. picenorum

Hieracium picroides Vill. subsp. christii (Arv.-Touv.) Zahn

Hieracium picroides Vill. subsp. grimsulanum (Zahn) Zahn

Hieracium picroides Vill. subsp. Iutescens (Huter ex Zahn) Greuter 
Hieracium picroides Vill. subsp. ochroleucum (W.D.J.Koch) Zahn

Hieracium picroides Vill. subsp. picroides

Hieracium picroides Vill. subsp. trichopicris (Zahn) Zahn

Hieracium pictum Pers. subsp. farinulentum (Jord.) Zahn

E Hieracium pictum Pers. subsp. irginianum (Arrigoni) Greuter

Hieracium pictum Pers. subsp. pictum

E Hieracium pietrae Zahn

Hieracium pietroszense Degen \& Zahn subsp. pietroszense

Hieracium pilosum Schleich. ex Froel. subsp. comatulum (Nägeli \& Peter) Gottschl.

Hieracium pilosum Schleich. ex Froel. subsp. favrei (Nägeli \& Peter) Greuter

Hieracium pilosum Schleich. ex Froel. subsp. Ianiceps (Nägeli \& Peter) Greuter

Hieracium pilosum Schleich. ex Froel. subsp. leucopogon (Zahn) Gottschl.

Hieracium pilosum Schleich. ex Froel. subsp. mollipellis (Zahn) Gottschl.

E Hieracium pilosum Schleich. ex Froel. subsp. padolanum (Pamp. \& Zahn) Gottschl.

Hieracium pilosum Schleich. ex Froel. subsp. pilosum

Hieracium pilosum Schleich. ex Froel. subsp. portae (Nägeli \& Peter) Gottschl.

Hieracium pilosum Schleich. ex Froel. subsp. schleicheri (Nägeli \& Peter) Greuter

Hieracium pilosum Schleich. ex Froel. subsp. sericotrichum (Nägeli \& Peter) Greuter

Hieracium pilosum Schleich. ex Froel. subsp. villosiceps Nägeli \& Peter ex Gottschl.

Hieracium pilosum Schleich. ex Froel. subsp. villosifolium (Nägeli \& Peter) Greuter

E Hieracium pioracense Gottschl.

E Hieracium pizense Zahn

Hieracium planchonianum Timb.-Lagr. \& Loret

Hieracium plantagineum (Arv.-Touv.) Arv.-Touv. subsp. gapense Zahn

Hieracium polatschekii Gottschl.

E Hieracium pollinense Zahn

E Hieracium pontiarnense Gottschl.

Hieracium porrectum $\mathrm{Fr}$. subsp. elongatiforme (Zahn) Zahn

Hieracium porrectum Fr. subsp. subelongatum (Nägeli \& Peter) Zahn

Hieracium porrifolium L. subsp. Iongibracteum Nägeli \& Peter

E Hieracium porrifolium L. subsp. niphanthum Nägeli \& Peter

Hieracium porrifolium L. subsp. porrifolium

Hieracium porrifolium L. subsp. pseudoporrifolium Nägeli \& Peter

Hieracium porrifolium L. subsp. sparsiglandulum Nägeli \& Peter

Hieracium portanum Belli

Hieracium pospichalii Zahn subsp. camunnorum Gottschl.

Hieracium pospichalii Zahn subsp. pospichalii

Hieracium pratorum-tivi Gottschl.

Hieracium predilense (Nägeli \& Peter) Zahn subsp. predilense

Hieracium predilense (Nägeli \& Peter) Zahn subsp. pseuderiopodum Zahn

Hieracium prenanthoides Vill. subsp. bupleurifolioides Zahn

Hieracium prenanthoides Vill. subsp. bupleurifolium (Tausch) Zahn

Hieracium prenanthoides Vill. subsp. cinereiceps (Zahn) Zahn

Hieracium prenanthoides Vill. subsp. constrictum (Arv.-Touv.) Zahn

E Hieracium prenanthoides Vill. subsp. costae Zahn ex Gottschl.

Hieracium prenanthoides Vill. subsp. cynanchoides (Arv.-Touv. \& Gaut.) Zahn

Hieracium prenanthoides Vill. subsp. jaquetianum (Zahn) Zahn

Hieracium prenanthoides Vill. subsp. juvonis (Murr) Zahn

Hieracium prenanthoides Vill. subsp. Ianceolatum (Vill.) Zahn

E Hieracium prenanthoides Vill. subsp. lissocorium Furrer \& Zahn

Hieracium prenanthoides Vill. subsp. perfoliatum (Froel.) Fr.

Hieracium prenanthoides Vill. subsp. praeruptorum Nyman

Hieracium prenanthoides Vill. subsp. prenanthoides

Hieracium prenanthoides Vill. subsp. strictissimum (Froel.) Zahn

Hieracium prenanthoides Vill. subsp. stupposifolium Gottschl.

Hieracium prenanthoides Vill. subsp. subelatifolium Zahn

Hieracium prenanthoides Vill. subsp. transalpinum (Arv.-Touv.) Zahn

E Hieracium prenanthoides Vill. subsp. valdegriseum Zahn

E Hieracium profetanum Belli

Hieracium pseudalpinum (Nägeli \& Peter) Prain subsp. cochlearioides Greuter

Hieracium pseudalpinum (Nägeli \& Peter) Prain subsp. pseudalpinum

E Hieracium pseudalpinum (Nägeli \& Peter) Prain subsp. pseudoschraderi (Dalla Torre \& Sarnth.) Greuter

Hieracium pseudalpinum (Nägeli \& Peter) Prain subsp. tissierei (Zahn) Greuter

E Hieracium pseudaustrale Gottschl.

Hieracium pseudocerinthe (Gaudin) W.D.J.Koch subsp. pseudocerinthe

Hieracium pseudocorymbosum Gremli

Hieracium pseudofritzei Benz \& Zahn

E Hieracium pseudogrovesianum Gottschl. subsp. amictum Gottschl.

Hieracium pseudogrovesianum Gottschl. subsp. circinans Gottschl.

Hieracium pseudogrovesianum Gottschl. subsp. leonense Gottschl.

Hieracium pseudogrovesianum Gottschl. subsp. opertum Gottschl.

Hieracium pseudogrovesianum Gottschl. subsp. pseudogrovesianum

Hieracium pseudopallidum Gottschl.

Hieracium pseudoprasinops Zahn subsp. pseudoprasinops

Hieracium pseudorionii (Zahn) Prain subsp. pictiforme (Zahn) Greuter

Hieracium pseudostenoplecum Zahn subsp. ochroleuciforme Murr \& Zahn

Hieracium pseudostenoplecum Zahn subsp. pseudostenoplecum

E Hieracium pteropogon Arv.-Touv. subsp. pogonites (Nägeli \& Peter) Zahn 
Hieracium pteropogon Arv.-Touv. subsp. pteropogon

Hieracium pujattii Gottschl.

Hieracium pulchellum Gren. ex Griseb. subsp. pleioscapum Zahn

Hieracium pulchellum Gren. ex Griseb. subsp. pulchellum

Hieracium racemosiforme (Zahn) Zahn

Hieracium racemosum Waldst. \& Kit. ex Willd. subsp. alismatifolium (Posp.) Zahn

Hieracium racemosum Waldst. \& Kit. ex Willd. subsp. apenninum (Levier ex Huter) Zahn

Hieracium racemosum Waldst. \& Kit. ex Willd. subsp. barbatum (Tausch ex Froel.) Zahn

Hieracium racemosum Waldst. \& Kit. ex Willd. subsp. caramanicum (Zahn) Zahn

Hieracium racemosum Waldst. \& Kit. ex Willd. subsp. crinitum (Sm.) Rouy

Hieracium racemosum Waldst. \& Kit. ex Willd. subsp. heterospermum (Arv.-Touv.) Zahn

Hieracium racemosum Waldst. \& Kit. ex Willd. subsp. italicum Fr. ex Zahn

Hieracium racemosum Waldst. \& Kit. ex Willd. subsp. Ieiopsis Murr \& Zahn

Hieracium racemosum Waldst. \& Kit. ex Willd. subsp. limbarae (Arrigoni) Greuter

Hieracium racemosum Waldst. \& Kit. ex Willd. subsp. moesiacum A.Kern. ex Zahn

E Hieracium racemosum Waldst. \& Kit. ex Willd. subsp. oliastrae (Arrigoni) Greuter

E Hieracium racemosum Waldst. \& Kit. ex Willd. subsp. pignattianum (Raimondo \& Di Grist.) Greuter

Hieracium racemosum Waldst. \& Kit. ex Willd. subsp. provinciale (Jord.) Rouy

E Hieracium racemosum Waldst. \& Kit. ex Willd. subsp. pulmonariifolium Gottschl.

Hieracium racemosum Waldst. \& Kit. ex Willd. subsp. racemosum

Hieracium racemosum Waldst. \& Kit. ex Willd. subsp. restonicanum Zahn

Hieracium racemosum Waldst. \& Kit. ex Willd. subsp. stiriacum (A.Kern. ex Willk.) Zahn

Hieracium racemosum Waldst. \& Kit. ex Willd. subsp. subhirsutum (Jord. ex Boreau) Zahn

Hieracium racemosum Waldst. \& Kit. ex Willd. subsp. sublateriflorum Zahn

Hieracium racemosum Waldst. \& Kit. ex Willd. subsp. substramineum Zahn

Hieracium racemosum Waldst. \& Kit. ex Willd. subsp. tenuifolium (Host) Zahn

Hieracium racemosum Waldst. \& Kit. ex Willd. subsp. virgaurea (Coss.) Zahn

E Hieracium ragognae Gottschl.

Hieracium ramosissimum Schleich. ex Hegetschw. subsp. adenoclinum (Arv.-Touv.) Zahn

Hieracium ramosissimum Schleich. ex Hegetschw. subsp. conringiifolium (Arv.-Touv.) Zahn

Hieracium ramosissimum Schleich. ex Hegetschw. subsp. cryptadenophyllum Zahn

E Hieracium ramosissimum Schleich. ex Hegetschw. subsp. garibaldianum (Fr.) Zahn

Hieracium ramosissimum Schleich. ex Hegetschw. subsp. Iactucifolium (Arv.-Touv.) Rouy

Hieracium ramosissimum Schleich. ex Hegetschw. subsp. ramosissimum

Hieracium ramosissimum Schleich. ex Hegetschw. subsp. scariolaceum (Arv.-Touv.) Zahn

Hieracium rapunculoides Arv.-Touv. subsp. contractum (Arv.-Touv.) Zahn

Hieracium rapunculoides Arv.-Touv. subsp. rapunculoides

E Hieracium rapunculoides Arv.-Touv. subsp. tridentatiflorum Zahn

Hieracium rohacsense Kit. subsp. bifidellum (Zahn) Zahn

Hieracium rohacsense Kit. subsp. furculaceum Zahn

Hieracium rohacsense Kit. subsp. pseudarolae (Zahn) Zahn

Hieracium rohacsense Kit. subsp. rauzense (Murr) Gottschl.

Hieracium rohacsense Kit. subsp. semiatratum (Zahn) Zahn

Hieracium rohacsense Kit. subsp. subsenile (Zahn) Zahn

E Hieracium rostanii Nägeli \& Peter subsp. bedolense Zahn

Hieracium rostanii Nägeli \& Peter subsp. centroalpinum Gottschl. \& Brandst.

E Hieracium rottii Gottschl.

Hieracium rupestre All.

Hieracium rupicola Jord.

E Hieracium rupicoliforme Zahn subsp. curunense Gottschl.

Hieracium sabaudum L. subsp. concinnum (Jord.) Zahn

E Hieracium sabaudum L. subsp. cumuliflorum (Zahn) Gottschl.

Hieracium sabaudum L. subsp. curvidens (Jord.) Zahn

Hieracium sabaudum L. subsp. dumosum (Jord.) Zahn

Hieracium sabaudum L. subsp. eminens (Jord. ex Sudre) Zahn

Hieracium sabaudum $\mathrm{L}$. subsp. fruticetorum (Jord. ex Boreau) Zahn

Hieracium sabaudum L. subsp. grandidentatum (Jord. ex Boreau) Zahn

Hieracium sabaudum L. subsp. nemorivagum (Jord. ex Boreau) Zahn

Hieracium sabaudum L. subsp. obliquum (Jord.) Zahn

Hieracium sabaudum L. subsp. occitanicum (Jord.) Zahn

Hieracium sabaudum L. subsp. propinquum (Sudre) Greuter

Hieracium sabaudum L. subsp. quercetorum (Jord. ex Boreau) Zahn

Hieracium sabaudum L. subsp. rigens (Jord.) Zahn

Hieracium sabaudum L. subsp. rigidicaule (Jord. ex Sudre) Zahn

Hieracium sabaudum L. subsp. roffavieri (Jord. ex Sudre) Zahn

Hieracium sabaudum L. subsp. sabaudiforme Zahn

Hieracium sabaudum L. subsp. sabaudum

Hieracium sabaudum L. subsp. salicetorum (Sudre) Zahn

Hieracium sabaudum L. subsp. scabiosum (Sudre) Zahn

Hieracium sabaudum L. subsp. sedunense Gremli ex Zahn

Hieracium sabaudum L. subsp. sublactucaceum Zahn

Hieracium sabaudum L. subsp. vagum (Jord.) Zahn

Hieracium sabaudum L. subsp. virescens (Sond.) Zahn

Hieracium sabaudum L. subsp. virgultorum (Jord.) Zahn

Hieracium saxatile Jacq. subsp. saxatile

Hieracium saxifragum Fr. subsp. sempronianum (Favrat) Zahn

Hieracium saxifragum Fr. subsp. vulpii Zahn

Hieracium schmidtii Tausch subsp. brunelliforme (Arv.-Touv.) O.Bolòs \& Vigo 
E Hieracium schmidtii Tausch subsp. catriae Gottschl.

Hieracium schmidtii Tausch subsp. ceratodon (Arv.-Touv.) O. Bolòs \& Vigo

E Hieracium schmidtii Tausch subsp. cochleariforme Gottschl.

E Hieracium schmidtii Tausch subsp. comatulum (Jord. ex Boreau) O. Bolòs \& Vigo

E Hieracium schmidtii Tausch subsp. comosulum (Arv.-Touv. \& Gaut.) O. Bolòs \& Vigo

E Hieracium schmidtii Tausch subsp. crinitisquamum Gottschl.

Hieracium schmidtii Tausch subsp. cyaneum (Arv.-Touv.) Zahn

Hieracium schmidtii Tausch subsp. didymum Zahn

Hieracium schmidtii Tausch subsp. glaucopallens (Zahn) O. Bolòs \& Vigo

Hieracium schmidtii Tausch subsp. Iasiophyllum (W.D.J.Koch) O.Bolòs \& Vigo

E Hieracium schmidtii Tausch subsp. madoniense (Raimondo \& Di Grist.) Greuter

E Hieracium schmidtii Tausch subsp. marchettii Gottschl.

E Hieracium schmidtii Tausch subsp. nebrodense (Tineo ex Lojac.) Di Grist., Gottschl. \& Raimondo

Hieracium schmidtii Tausch subsp. rupicola Fr. ex Zahn

Hieracium schmidtii Tausch subsp. schmidtii

Hieracium schmidtii Tausch subsp. schmidtiiforme (Zahn) O.Bolòs \& Vigo

Hieracium schmidtii Tausch subsp. subcomatulum (Zahn) O.Bolòs \& Vigo

Hieracium schmidtii Tausch subsp. subrupicola Zahn

E Hieracium scorzonerifolium Vill. subsp. antelaonis Pamp. \& Zahn

E Hieracium scorzonerifolium Vill. subsp. divaricatum Huter, Porta \& Rigo ex Nägeli \& Peter

Hieracium scorzonerifolium Vill. subsp. flexuosum Waldst. \& Kit. ex Nägeli \& Peter

Hieracium scorzonerifolium Vill. subsp. heterocephalum Nägeli \& Peter

Hieracium scorzonerifolium Vill. subsp. Ionchophylloides Zahn ex Dalla Torre \& Sarnth.

Hieracium scorzonerifolium Vill. subsp. myocomum Nägeli \& Peter

E Hieracium scorzonerifolium Vill. subsp. nudissimum Gottschl.

Hieracium scorzonerifolium Vill. subsp. pantotrichum Nägeli \& Peter

Hieracium scorzonerifolium Vill. subsp. polybracteum Nägeli \& Peter

Hieracium scorzonerifolium Vill. subsp. scorzonerifolium

Hieracium scorzonerifolium Vill. subsp. speciosiforme Nägeli \& Peter

Hieracium segureum Arv.-Touv. subsp. cirritiforme Zahn

E Hieracium segureum Arv.-Touv. subsp. exilentoides Zahn

E Hieracium segureum Arv.-Touv. subsp. rivifrigidi Zahn

Hieracium segureum Arv.-Touv. subsp. salassorum (Besse) Zahn

E Hieracium segusianum Gottschl.

E Hieracium semicanescens Gottschl.

E Hieracium semipallescens Gottschl.

E Hieracium serratum Nägeli \& Peter subsp. serratum

E Hieracium silsinum Nägeli \& Peter subsp. subsilsinum (Zahn) Zahn

E Hieracium simbruinicum Gottschl.

Hieracium simia (Huter ex Zahn) Zahn subsp. simia

Hieracium simia (Huter ex Zahn) Zahn subsp. subincertum Zahn

E Hieracium sparsiramum Nägeli \& Peter subsp. ampezzanum (Nägeli \& Peter) Gottschl.

E Hieracium sparsivestitum Gottschl.

Hieracium sparsum Friv. subsp. vierhapperi Zahn

Hieracium speciosum Willd. ex Hornem.

Hieracium spectabile Fr. ex Arv.-Touv.

E Hieracium squarrosofurcatum Gottschl.

E Hieracium staui Belli

Hieracium stenoplecum Arv.-Touv. \& Huter subsp. stenoplecum

Hieracium sterzingense Zahn

E Hieracium strafforelloanum Zahn

Hieracium subcaesiiforme (Zahn) Prain subsp. subcaesiiforme

Hieracium subpamphilei Zahn

Hieracium substellatum Arv.-Touv. \& Gaut.

Hieracium subtilissimum Zahn subsp. subtilissimum

Hieracium subtomentosum (Burnat \& Gremli) Zahn

Hieracium symphytaceum Arv.-Touv. subsp. elocatum (Huter ex Gottschl.) Gottschl.

Hieracium symphytaceum Arv.-Touv. subsp. neoprenanthes (Arv.-Touv.) Zahn

E Hieracium symphytaceum Arv.-Touv. subsp. odontinum (Arv.-Touv. ex Belli) Zahn

Hieracium symphytaceum Arv.-Touv. subsp. pseudoleiopsis (Murr) Gottschl. \& Brandst.

Hieracium symphytaceum Arv.-Touv. subsp. rotgesianum (Arv.-Touv. \& Gaut.) Zahn

E Hieracium symphytaceum Arv.-Touv. subsp. sarracenicum (Arv.-Touv. \& Belli) Zahn

Hieracium symphytaceum Arv.-Touv. subsp. symphytaceum

E Hieracium symphytifolium Froel.

Hieracium taurinense Jord. subsp. taurinense

E Hieracium taurinense Jord. subsp. taurinorum (Zahn) Zahn

Hieracium tenuiflorum Arv.-Touv. subsp. austrosilvularum (P.Rossi \& Zahn) Gottschl.

Hieracium tenuiflorum Arv.-Touv. subsp. glaucoviolascens (Bornm. \& Zahn) Gottschl.

E Hieracium tenuiflorum Arv.-Touv. subsp. pictoprasinum (Fen. \& Zahn) Gottschl.

Hieracium tenuiflorum Arv.-Touv. subsp. pseudomerianum (P.Rossi \& Zahn) Gottschl.

E Hieracium tenuiflorum Arv.-Touv. subsp. sebini (Fen. \& Zahn) Gottschl.

Hieracium tenuiflorum Arv.-Touv. subsp. tenuiflorum

Hieracium tephrodermum Zahn subsp. subexpansum (Zahn) Zahn

E Hieracium tephropogon Zahn subsp. gortanianum (Arv.-Touv. \& Belli) Gottschl.

Hieracium tephropogon Zahn subsp. subintermedium Zahn

Hieracium tephropogon Zahn subsp. tephropogon

Hieracium tephrosoma (Nägeli \& Peter) Zahn subsp. brachypogon (Zahn) Zahn

Hieracium tephrosoma (Nägeli \& Peter) Zahn subsp. kuekenthalianum (Zahn) Zahn 
Hieracium tephrosoma (Nägeli \& Peter) Zahn subsp. tephrosoma

Hieracium tephrosoma (Nägeli \& Peter) Zahn subsp. trichothecum (Zahn ex Dalla Torre \& Sarnth.) Zahn

Hieracium tephrosoma (Nägeli \& Peter) Zahn subsp. trivialiforme Zahn

Hieracium terraccianoi Di Grist., Gottschl. \& Raimondo

Hieracium thesauranum Gottschl.

Hieracium thesioides Gottschl.

Hieracium tolstoii Fen. \& Zahn

Hieracium tomentosum L. subsp. andryaloides (Vill.) Nägeli \& Peter

Hieracium tomentosum L. subsp. gnaphalodes (Arv.-Touv.) Greuter

Hieracium tomentosum L. subsp. liotardii (Vill.) Zahn

Hieracium tomentosum L. subsp. phlomidifolium (Arv.-Touv.) Nägeli \& Peter

Hieracium tomentosum $\mathrm{L}$. subsp. tomentosum

Hieracium tonalense Gottschl.

Hieracium torrigliense Gottschl.

Hieracium toscoemilianum Gottschl.

Hieracium toutonianum (Zahn) Zahn

E Hieracium truttae Gottschl.

Hieracium umbellatum $L$. subsp. brevifolioides Zahn

Hieracium umbellatum $L$. subsp. umbellatum

E Hieracium umbrosoides Gottschl.

Hieracium umbrosum Jord. subsp. abietinum (Boiss. \& Heldr.) Greuter

Hieracium umbrosum Jord. subsp. acrotephrophorum (Zahn) Zahn

Hieracium umbrosum Jord. subsp. crepidifolium (Arv.-Touv.) Zahn

E Hieracium umbrosum Jord. subsp. mindinoicum Zahn

E Hieracium umbrosum Jord. subsp. oleicolor (Zahn) Greuter

Hieracium umbrosum Jord. subsp. pseudofastigiatum (Degen \& Zahn) Zahn

Hieracium umbrosum Jord. subsp. subexilentum Zahn

Hieracium umbrosum Jord. subsp. umbrosum

E Hieracium umbrosum Jord. subsp. vippetinum (Huter) Zahn

E Hieracium umbrosum Jord. subsp. visaillense Paléz. \& Zahn

Hieracium urticaceum Arv.-Touv. \& Ravaud subsp. amplexicauliforme Zahn

Hieracium urticaceum Arv.-Touv. \& Ravaud subsp. urticaceum

Hieracium valdepilosum Vill. subsp. calvulum (Nägeli \& Peter) Zahn

Hieracium valdepilosum Vill. subsp. christeneri (Nägeli \& Peter) Zahn

Hieracium valdepilosum Vill. subsp. elongatum Willd. ex Zahn

Hieracium valdepilosum Vill. subsp. interjectum (Beck) Zahn

Hieracium valdepilosum Vill. subsp. oligophyllum (Nägeli \& Peter) Zahn

Hieracium valdepilosum Vill. subsp. pseudelongatum (Nägeli \& Peter) Zahn

Hieracium valdepilosum Vill. subsp. raphiolepium (Nägeli \& Peter) Zahn

Hieracium valdepilosum Vill. subsp. subalpinum (Nägeli \& Peter) Greuter

Hieracium valdepilosum Vill. subsp. subsinuatum (Nägeli \& Peter) Zahn

Hieracium valdepilosum Vill. subsp. valdepilosum

E Hieracium valoddae (Zahn) Prain subsp. austroitalicum (Zahn) Zahn

Hieracium valoddae (Zahn) Prain subsp. knautiifolium (Arv.-Touv.) Gottschl.

Hieracium valoddae (Zahn) Prain subsp. raccolanae (Zahn) Zahn

E Hieracium vasconicum Martrin-Donos subsp. dolosum (Burnat \& Gremli) Greuter

Hieracium vasconicum Martrin-Donos subsp. Iaureolum (Arv.-Touv.) Greuter

Hieracium vasconicum Martrin-Donos subsp. vasconicum

E Hieracium venostorum (Zahn) Gottschl.

E Hieracium venticaesum Gottschl.

E Hieracium verbascifolium Vill. subsp. capreifolium (Nägeli \& Peter) Zahn

E Hieracium verbascifolium Vill. subsp. linguiforme (Nägeli \& Peter) Zahn

Hieracium verbascifolium Vill. subsp. mallobrachion Zahn

E Hieracium verbascifolium Vill. subsp. mallophorum (Nägeli \& Peter) Zahn

Hieracium verbascifolium Vill. subsp. melandrifolium (Arv.-Touv.) Zahn

Hieracium verbascifolium Vill. subsp. menthifolium (Arv.-Touv.) Zahn

Hieracium verbascifolium Vill. subsp. thapsoides (Arv.-Touv.) Zahn

Hieracium verbascifolium Vill. subsp. verbascifolium

Hieracium vetteri Ronniger

Hieracium villosum Jacq. subsp. albolanatum Nägeli \& Peter

Hieracium villosum Jacq. subsp. calvifolium Nägeli \& Peter

Hieracium villosum Jacq. subsp. doratophyllum Nägeli \& Peter

Hieracium villosum Jacq. subsp. eurybasis Nägeli \& Peter

Hieracium villosum Jacq. subsp. glaucifrons Nägeli \& Peter

Hieracium villosum Jacq. subsp. heterophyllum Nägeli \& Peter

Hieracium villosum Jacq. subsp. ovalifolium Nägeli \& Peter

Hieracium villosum Jacq. subsp. undulifolium Nägeli \& Peter

Hieracium villosum Jacq. subsp. villosissimum (Nägeli) Nägeli \& Peter

Hieracium villosum Jacq. subsp. villosum

Hieracium viride Arv.-Touv. subsp. caerulaceum (Arv.-Touv.) Zahn

Hieracium viride Arv.-Touv. subsp. intricatum (Arv.-Touv.) Zahn

E Hieracium viride Arv.-Touv. subsp. penninicola (Besse \& Zahn) Zahn

Hieracium viride Arv.-Touv. subsp. viride

Hieracium viscosum Arv.-Touv. subsp. Iactucoides Zahn

E Hieracium viscosum Arv.-Touv. subsp. viscosiforme Zahn

Hieracium viscosum Arv.-Touv. subsp. viscosum

E Hieracium volaiense Gottschl.

Hieracium vollmannii Zahn subsp. grimsulicola Zahn 
Hieracium vollmannii Zahn subsp. vollmannii

Hieracium wilczekianum Arv.-Touv. subsp. bagnense (Zahn) Zahn

Hieracium wilczekianum Arv.-Touv. subsp. matajurense (Zahn) Zahn

Hieracium wilczekianum Arv.-Touv. subsp. trigonolepium Zahn

Homogyne alpina (L.) Cass.

Homogyne discolor (Jacq.) Cass.

Homogyne sylvestris Cass.

Hyoseris lucida L. subsp. taurina (Martinoli) Peruzzi \& Vangelisti

Hyoseris radiata $\mathrm{L}$.

Hyoseris scabra $\mathrm{L}$.

Hypochaeris achyrophorus $\mathrm{L}$.

Hypochaeris cretensis (L.) Bory \& Chaub.

Hypochaeris facchiniana Ambrosi

Hypochaeris glabra $\mathrm{L}$.

Hypochaeris Iaevigata (L.) Ces., Pass. \& Gibelli

Hypochaeris maculata $\mathrm{L}$.

Hypochaeris radicata $\mathrm{L}$.

ET Hypochaeris sardoa Bacch., Brullo \& Terrasi

Hypochaeris uniflora Vill.

Inula bifrons L.

Inula britannica L. subsp. britannica

Inula conyzae (Griess.) DC.

Inula ensifolia $\mathrm{L}$.

Inula helenium L. subsp. helenium

Inula helvetica Weber

Inula hirta L.

Inula montana $\mathrm{L}$.

Inula salicina $\mathrm{L}$.

Inula spiraeifolia L.

Inula verbascifolia (Willd.) Hausskn.

Jacobaea abrotanifolia (L.) Moench subsp. abrotanifolia

Jacobaea abrotanifolia (L.) Moench subsp. tiroliensis (A.Kern. ex Dalla Torre) B.Nord. \& Greuter

Jacobaea alpina (L.) Moench subsp. alpina

E Jacobaea alpina (L.) Moench subsp. samnitum (Nyman) Peruzzi

E Jacobaea ambigua (Biv.) Pelser \& Veldkamp subsp. ambigua

E Jacobaea ambigua (Biv.) Pelser \& Veldkamp subsp. nebrodensis (Guss.) Peruzzi \& N.G.Passal. \& C.E.Jarvis

Jacobaea aquatica (Hill) G.Gaertn., B.Mey. \& Scherb.

Jacobaea carniolica (Willd.) Schrank

Jacobaea delphiniifolia (Vahl) Pelser \& Veldkamp

Jacobaea disjuncta (Flatscher, Schneew. \& Schönsw.) Galasso \& Bartolucc

Jacobaea erratica (Bertol.) Fourr.

Jacobaea erucifolia (L.) G.Gaertn., B.Mey. \& Scherb. subsp. erucifolia

Jacobaea erucifolia (L.) G.Gaertn., B.Mey. \& Scherb. subsp. praealta (Bertol.) Greuter \& B.Nord.

Jacobaea erucifolia (L.) G.Gaertn., B.Mey. \& Scherb. subsp. tenuifolia (J.Presl \& C.Presl) B.Nord. \& Greuter

Jacobaea incana (L.) Veldkamp

Jacobaea insubrica (Chenevard) Galasso \& Bartolucci

E Jacobaea lycopifolia (Desf. ex Poir.) Greuter \& B.Nord.

E Jacobaea maritima (L.) Pelser \& Meijden subsp. bicolor (Willd.) B.Nord. \& Greuter

E Jacobaea maritima (L.) Pelser \& Meijden subsp. gibbosa (Guss.) Peruzzi \& N.G.Passal. \& C.E.Jarvis

Jacobaea maritima (L.) Pelser \& Meijden subsp. maritima

Jacobaea maritima (L.) Pelser \& Meijden subsp. sicula N.G.Passal., Peruzzi \& Pellegrino

Jacobaea paludosa (L.) G.Gaertn., B.Mey. \& Scherb. subsp. paludosa

Jacobaea persoonii (De Not.) Pelser

Jacobaea uniflora (All.) Veldkamp

Jacobaea vulgaris Gaertn. subsp. gotlandica (Neuman) B.Nord. - Note: For the Italian distribution of this taxon, see Conti et al. (2012).

Jacobaea vulgaris Gaertn. subsp. vulgaris

E Jurinea bocconei (Guss.) Guss.

Jurinea mollis (L.) Rchb. subsp. mollis

E Klasea flavescens (L.) Holub subsp. cichoracea (L.) Greuter \& Wagenitz

Klasea flavescens (L.) Holub subsp. mucronata (Desf.) Cantó \& Rivas Mart.

Klasea lycopifolia (Vill.) Á.Löve \& D.Löve

Klasea nudicaulis (L.) Fourr.

Klasea radiata (Waldst. \& Kit.) Á.Löve \& D.Löve subsp. radiata

Lactuca longidentata Moris

Lactuca perennis L.

Lactuca quercina L. subsp. quercina

Lactuca saligna $\mathrm{L}$.

Lactuca sativa L. subsp. serriola (L.) Galasso, Banfi, Bartolucci \& Ardenghi

Lactuca tenerrima Pourr.

T Lactuca viminea (L.) J.Presl \& C.Presl subsp. chondrilliflora (Boreau) St.-Lag.

T Lactuca viminea (L.) J.Presl \& C.Presl subsp. ramosissima (All.) Arcang.

Lactuca viminea (L.) J.Presl \& C.Presl subsp. viminea

Lactuca virosa $\mathrm{L}$.

E Lamyropsis microcephala (Moris) Dittrich \& Greuter

Lapsana communis $L$. subsp. communis

Launaea fragilis (Asso) Pau

Launaea nudicaulis (L.) Hook.f. - Note : Possibly extinct in Italy.

Leontodon anomalus Ball 
Leontodon apulus (Fiori) Brullo

Leontodon berinii (Bartl.) Roth

Leontodon crispus Vill.

Leontodon hispidus L. subsp. dubius (Hoppe) Pawłowska

Leontodon hispidus $L$. subsp. hispidus

Leontodon hispidus L. subsp. hyoseroides (Welw. ex Rchb.) Murr

Leontodon incanus (L.) Schrank

E Leontodon intermedius (Fiori) Huter, Porta \& Rigo ex Rigo

Leontodon rosanoi (Ten.) DC.

Leontodon saxatilis Lam. subsp. rothii Maire

Leontodon saxatilis Lam. subsp. saxatilis

E Leontodon siculus (Guss.) Nyman

Leontodon tenuiflorus (Gaudin) Rchb.

Leontodon tuberosus $\mathrm{L}$.

Leontopodium alpinum Cass.

Leontopodium nivale (Ten.) Hand.-Mazz.

Leucanthemopsis alpina (L.) Heywood subsp. alpina

Leucanthemopsis alpina (L.) Heywood subsp. minima (Vill.) Holub

Leucanthemum adustum (W.D.J.Koch) Gremli subsp. adustum

Leucanthemum coronopifolium Vill. subsp. ceratophylloides (All.) Vogt \& Greuter

Leucanthemum coronopifolium Vill. subsp. coronopifolium

Leucanthemum coronopifolium Vill. subsp. tenuifolium (Guss.) Vogt \& Greuter

Leucanthemum gaudinii Dalla Torre

Leucanthemum heterophyllum (Willd.) DC

T Leucanthemum ircutianum DC. subsp. asperulum (N.Terracc.) Vogt \& Greuter

Leucanthemum ircutianum DC. subsp. ircutianum

T Leucanthemum ircutianum DC. subsp. leucolepis (Briq. \& Cavill.) Vogt \& Greuter

E Leucanthemum laciniatum Huter, Porta \& Rigo

E Leucanthemum ligusticum Marchetti, R.Bernardello, Melai \& Peruzzi - Note: The records of L. legraeanum (Rouy) B.Bock \& J.-M.Tison from LIG and EMR should

be referred to $L$. Xaramisii Florian Wagner, Vogt \& Oberpr. [L. ligusticum $\times$ L. vulgare] (Wagner et al. 2017).

T Leucanthemum pachyphyllum Marchi \& Illuminati

Leucanthemum pallens (J.Gay ex Perreym.) DC.

Leucanthemum platylepis Borbás

Leucanthemum subglaucum De Laramb.

E Leucanthemum tridactylites (A.Kern. \& Huter ex Porta \& Rigo) Huter, Porta \& Rigo

Leucanthemum virgatum (Desr.) Clos

Leucanthemum vulgare (Vaill.) Lam. subsp. vulgare

Limbarda crithmoides (L.) Dumort. subsp. longifolia (Arcang.) Greuter

Logfia gallica (L.) Cosson \& Germ.

Logfia heterantha (Raf.) J.Holub

Logfia lojaconoi (Brullo) C.Brullo \& Brullo

Logfia minima (Sm.) Dumort.

Lonas annua (L.) Vines \& Druce

Mantisalca duriaei (Spach) Briq. \& Cavill.

Mantisalca salmantica (L.) Briq. \& Cavill.

Matricaria aurea (Loefl.) Sch.Bip.

Matricaria chamomilla $\mathrm{L}$.

Mycelis muralis (L.) Dumort. subsp. muralis

Nananthea perpusilla (Loisel.) DC.

Notobasis syriaca (L.) Cass.

Omalotheca diminuta (Braun-Blanq.) Bartolucci \& Galasso

Omalotheca hoppeana (W.D.J.Koch) Sch.Bip. \& F.W.Schultz

Omalotheca norvegica (Gunnerus) Sch.Bip. \& F.W.Schultz

Omalotheca supina (L.) DC.

Omalotheca sylvatica (L.) Sch.Bip. \& F.W.Schultz

Onopordum acanthium $L$. subsp. acanthium

Onopordum horridum Viv.

Onopordum illyricum L. subsp. cardunculus (Boiss.) Arènes

Onopordum illyricum L. subsp. illyricum

Onopordum macracanthum Schousb.

Onopordum tauricum Willd.

Pallenis maritima (L.) Greuter

Pallenis spinosa (L.) Cass. subsp. spinosa

Petasites albus (L.) Gaertn.

Petasites hybridus (L.) G.Gaertn., B.Mey. \& Scherb. subsp. hybridus

Petasites paradoxus (Retz.) Baumg.

Petasites pyrenaicus (L.) G.López

Phagnalon rupestre (L.) DC. subsp. illyricum (H.Lindb.) Ginzb.

Phagnalon rupestre (L.) DC. subsp. morisianum (Ces., Pass. \& Gibelli) Arcang.

Phagnalon rupestre (L.) DC. subsp. rupestre

Phagnalon saxatile (L.) Cass.

Phagnalon sordidum (L.) Rchb.

Picnomon acarna (L.) Cass.

Picris hieracioides L. subsp. hieracioides

Picris hieracioides L. subsp. umbellata (Schrank) Ces.

Picris hispidissima (Bartl.) W.D.J.Koch 
Pilosella acutifolia (Vill.) Arv.-Touv.

Pilosella aletschensis (Zahn) Soják

Pilosella alpicola (Schleich. ex Steud. \& Hochst.) F.W.Schultz \& Sch.Bip.

Pilosella amphipolia (Nägeli \& Peter) Gottschl.

Pilosella anchusoides Arv.-Touv.

Pilosella anobrachia (Arv.-Touv. \& Gaut.) S.Bräut. \& Greuter

Pilosella arida (Freyn) Soják

Pilosella arnoserioides (Nägeli \& Peter) Soják

Pilosella aurantella (Nägeli \& Peter) Soják

Pilosella aurantiaca (L.) F.W.Schultz \& Sch.Bip. subsp. aurantiaca

Pilosella aurantiaca (L.) F.W.Schultz \& Sch.Bip. subsp. auropurpurea (Peter) Soják

Pilosella aurantiaca (L.) F.W.Schultz \& Sch.Bip. subsp. decolorans (Fr.) T.Tyler

Pilosella auriculiformis (Fr.) F.W.Schultz \& Sch.Bip.

Pilosella basifurca (Peter) Soják

Pilosella bauhini (Schult.) Arv.-Touv. subsp. magyarica (Peter) S.Bräut.

Pilosella blyttiana (Fr.) F.W.Schultz \& Sch.Bip.

Pilosella brachycoma (Nägeli \& Peter) H.P.Fuchs

Pilosella caespitosa (Dumort.) P.D.Sell \& C.West

E Pilosella calabra (Nägeli \& Peter) Soják

Pilosella calodon (Tausch ex Peter) Soják

E Pilosella cepitina (Gottschl.) Gottschl.

Pilosella cinerosiformis (Nägeli \& Peter) Gottschl.

E Pilosella corvigena (Gottschl.) Gottschl.

Pilosella corymbuloides (Arv.-Touv.) S.Bräut. \& Greuter

Pilosella cymiflora (Nägeli \& Peter) S.Bräut. \& Greuter

Pilosella cymosa (L.) F.W.Schultz \& Sch.Bip. subsp. cymosa

Pilosella cymosa (L.) F.W.Schultz \& Sch.Bip. subsp. sabina (Sebast.) H.P.Fuchs

Pilosella cymosa (L.) F.W.Schultz \& Sch.Bip. subsp. vaillantii (Tausch) S. Bräut. \& Greuter

Pilosella densiflora (Tausch) Soják

Pilosella fainensis Soest ex Gottschl.

Pilosella fallacina (F.W.Schultz) F.W.Schultz

Pilosella faurei Arv.-Touv.

Pilosella frigidaria (Nägeli \& Peter) Soják

Pilosella fulviseta (Bertol.) Soják

Pilosella fusca (Vill.) Arv.-Touv.

Pilosella glacialis (Reyn. ex Lachen.) F.W.Schultz \& Sch.Bip.

Pilosella glaciella (Nägeli \& Peter) Soják

Pilosella guthnikiana (Hegetschw.) Soják

Pilosella hoppeana (Schult.) F.W.Schultz \& Sch.Bip. subsp. hoppeana

Pilosella hoppeana (Schult.) F.W.Schultz \& Sch.Bip. subsp. macrantha (Ten.) S.Bräut. \& Greuter

E Pilosella hoppeana (Schult.) F.W.Schultz \& Sch.Bip. subsp. sicula Di Grist., Gottschl. \& Raimondo

E Pilosella huberi (Zahn) Gottschl.

Pilosella hybrida (Chaix) F.W.Schultz \& Sch.Bip.

Pilosella hypeurya (Peter) Soják

Pilosella hypoleuca Arv.-Touv.

Pilosella kalksburgensis (Wiesb.) Soják

E Pilosella kralikii (Rouy) J.-M.Tison

Pilosella lactucella (Wallr.) P.D.Sell \& C.West subsp. lactucella

Pilosella lactucella (Wallr.) P.D.Sell \& C.West subsp. nana (Scheele) M.Laínz

Pilosella laggeri (Sch.Bip. ex Rchb.f.) F.W.Schultz \& Sch.Bip.

Pilosella lathraea (Peter) Soják

Pilosella leptophyton (Nägeli \& Peter) S.Bräut. \& Greuter

Pilosella leucopsilon (Arv.-Touv.) Gottschl.

Pilosella litardiereana (Zahn) Soják

Pilosella longisquama (Peter) Holub

E Pilosella macranthiformis (Zahn) S.Bräut. \& Greuter

Pilosella macristolona (Nägeli \& Peter) Gottschl.

E Pilosella medioposita (Gottschl.) Gottschl.

E Pilosella neogelmii (Gottschl.) Gottschl.

Pilosella nigricarina (Nägeli \& Peter) Soják

Pilosella notha (Huter) S.Bräut. \& Greuter

Pilosella officinarum Vaill.

E Pilosella pachycymigera (Gottschl.) Gottschl.

Pilosella pachypila (Peter) Soják

Pilosella peleteriana (Mérat) F.W.Schultz \& Sch.Bip. subsp. peleteriana

Pilosella peleteriana (Mérat) F.W.Schultz \& Sch.Bip. subsp. subpeleteriana (Nägeli \& Peter) P.D.Sell

Pilosella permutata (Nägeli \& Peter) Soják

Pilosella peteriana (Käser) Holub

Pilosella piloselloides (Vill.) Soják subsp. floccosa (Nägeli \& Peter) S.Bräut. \& Greuter

Pilosella piloselloides (Vill.) Soják subsp. litorale (Nägeli \& Peter) Zahn

Pilosella piloselloides (Vill.) Soják subsp. piloselloides

Pilosella piloselloides (Vill.) Soják subsp. praealta (Vill. ex Gochnat) S.Bräut. \& Greuter

Pilosella plaicensis (Woł.) Soják

Pilosella portae (Willk. ex T.Durand \& B.D.Jacks.) Mateo \& Greuter

Pilosella promeces (Peter) Holub

E Pilosella pseudolactucella Gottschl.

Pilosella pseudopilosella (Ten.) Soják

Pilosella pseudotrichodes (Zahn) Soják 
Pilosella rubra (Peter) Soják

Pilosella salernicola (J.Vetter \& Zahn) Soják

Pilosella saussureoides Arv.-Touv.

Pilosella schizosciadia Gottschl.

Pilosella schultesii (F.W.Schultz) F.W.Schultz \& Sch.Bip.

Pilosella sciadogena Gottschl.

Pilosella sciadophora (Nägeli \& Peter) Soják

Pilosella sphaerocephala (Froel. ex Rchb.) F.W.Schultz \& Sch.Bip.

Pilosella stoloniflora (Waldst. \& Kit.) F.W.Schultz \& Sch.Bip.

Pilosella subrubens Arv.-Touv.

Pilosella substoloniflora (Peter) Soják

Pilosella subtardans (Nägeli \& Peter) Soják

Pilosella sulphurea (Döll) F.W.Schultz \& Sch.Bip.

Pilosella tardiuscula (Peter) Soják

Pilosella tendina (Nägeli \& Peter) Soják

Pilosella tephrodes (Nägeli \& Peter) S.Bräut. \& Greuter

Pilosella tinctilingua (Zahn) Soják

Pilosella triplex (Peter) Soják

Pilosella velutina (Hegetschw.) F.W.Schultz \& Sch.Bip.

Pilosella viridifolia (Peter) Holub

Pilosella visianii F.W.Schultz \& Sch.Bip.

Pilosella ziziana (Tausch) F.W.Schultz \& Sch.Bip.

E Plagius flosculosus (L.) Alavi \& Heywood

Podospermum canum C.A.Mey.

Podospermum laciniatum (L.) DC. subsp. decumbens (Guss.) Gemeinholzer \& Greuter

Podospermum laciniatum (L.) DC. subsp. Iaciniatum

Podospermum purpureum (L.) W.D.J.Koch \& Ziz

Podospermum roseum (Waldst. \& Kit.) Gemeinholzer \& Greuter

Prenanthes purpurea $\mathrm{L}$.

Ptilostemon casabonae (L.) Greuter

Ptilostemon gnaphaloides (Cirillo) Soják subsp. gnaphaloides

E Ptilostemon greuteri Raimondo \& Domina

E Ptilostemon niveus (C.Presl) Greuter

Ptilostemon stellatus (L.) Greuter

Ptilostemon strictus (Ten.) Greuter

Pulicaria dysenterica (L.) Bernh.

Pulicaria odora (L.) Rchb.

Pulicaria sicula (L.) Moris

Pulicaria vulgaris Gaertn.

Reichardia intermedia (Sch.Bip.) Samp.

Reichardia picroides (L.) Roth

Reichardia tingitana (L.) Roth

Rhagadiolus edulis Gaertn.

Rhagadiolus stellatus (L.) Gaertn.

Rhaponticoides africana (Lam.) M.V.Agab. \& Greuter

Rhaponticoides alpina (L.) M.V.Agab. \& Greuter

Rhaponticoides centaurium (L.) M.V.Agab. \& Greuter - Note: According to Bartolucci et al. (2017a), R. calabrica Puntillo \& Peruzzi is considered here as a heterotypic synonym of this species.

Rhaponticum bicknellii (Briq.) Banfi, Galasso \& Soldano

Rhaponticum coniferum (L.) Greuter

Rhaponticum hemeralpion Banfi, Galasso \& Soldano - Note: According to Banfi et al. (2011), R. heleniifolium Godr. \& Gren. should be excluded from the Italian flora.

Rhaponticum scariosum Lam.

Robertia taraxacoides (Loisel.) DC.

Santolina corsica Jord. \& Fourr.

Santolina etrusca (Lacaita) Marchi \& D'Amato

Santolina insularis (Gennari ex Fiori) Arrigoni

Santolina ligustica Arrigoni

Santolina neapolitana Jord. \& Fourr.

Santolina pinnata Viv.

Saussurea alpina (L.) DC. subsp. alpina

Saussurea alpina (L.) DC. subsp. depressa (Gren.) Nyman

Saussurea discolor (Willd.) DC.

Saussurea pygmaea (Jacq.) Spreng.

Schlagintweitia huteri (Hausm. ex Bamb.) Gottschl. \& Greuter subsp. huteri

Schlagintweitia huteri (Hausm. ex Bamb.) Gottschl. \& Greuter subsp. Iantoscana (Burnat \& Gremli) Gottschl.

Schlagintweitia intybacea (All.) Griseb.

Scolymus grandiflorus Desf.

Scolymus hispanicus L. subsp. hispanicus

Scolymus hispanicus L. subsp. occidentalis F.M.Vásquez

Scolymus maculatus L.

Scorzonera aristata Ramond ex DC.

Scorzonera austriaca Willd.

E Scorzonera callosa Moris

Scorzonera hirsuta (Gouan) L.

Scorzonera hispanica L. subsp. asphodeloides (Wallr.) Arcang.

Scorzonera hispanica L. subsp. hispanica

Scorzonera hispanica L. subsp. neapolitana (Grande) Greuter 
Scorzonera humilis L.

Scorzonera undulata Vahl subsp. deliciosa (Guss. ex DC.) Maire

E Scorzonera villosa Scop. subsp. columnae (Guss.) Nyman

Scorzonera villosa Scop. subsp. villosa

Scorzoneroides autumnalis (L.) Moench

Scorzoneroides cichoriacea (Ten.) Greuter

Scorzoneroides helvetica (Mérat) Holub

E Scorzoneroides montana (Lam.) Holub subsp. breviscapa (DC.) Greuter

Scorzoneroides montana (Lam.) Holub subsp. melanotricha (Vierh.) Gutermann

Scorzoneroides montana (Lam.) Holub subsp. montana

Scorzoneroides muelleri (Sch.Bip.) Greuter \& Talavera

Senecio altissimus Mill.

Senecio cacaliaster Lam.

Senecio doronicum (L.) L. subsp. doronicum

Senecio doronicum (L.) L. subsp. orientalis J.Calvo

Senecio fontanicola Grulich \& Hodálová

Senecio gallicus Vill.

Senecio glaucus L. subsp. coronopifolius (Maire) C.Alexander

E Senecio glaucus L. subsp. hyblaeus Brullo

Senecio hercynicus Herborg subsp. hercynicus

Senecio leucanthemifolius Poir. subsp. leucanthemifolius

Senecio leucanthemifolius Poir. subsp. mauritanicus (Pomel) Greuter

Senecio lividus $L$.

E Senecio morisii J.Calvo \& Bacch.

E Senecio nemorensis L. subsp. apuanus (Tausch) Greuter

Senecio nemorensis L. subsp. glabratus (Herborg) Oberpr.

Senecio nemorensis L. subsp. jacquinianus (Rchb.) Čelak.

Senecio ovatus (G.Gaertn., B.Mey. \& Scherb.) Willd. subsp. alpestris (Gaudin) Herborg

Senecio ovatus (G.Gaertn., B.Mey. \& Scherb.) Willd. subsp. ovatus

E Senecio ovatus (G.Gaertn., B.Mey. \& Scherb.) Willd. subsp. stabianus (Lacaita) Greuter

Senecio petraeus Boiss. \& Reut.

Senecio provincialis (L.) Druce

E Senecio scopolii Hoppe \& Hornsch. subsp. floccosus (Bertol.) Greuter

Senecio scopolii Hoppe \& Hornsch. subsp. scopolii

E Senecio squalidus L. subsp. aethnensis (Jan ex DC.) Greuter

Senecio squalidus L. subsp. aurasicus (Batt.) C.Alexander

Senecio squalidus L. subsp. calabrus (Fiori) Peruzzi \& Bernardo

Senecio squalidus L. subsp. chrysanthemifolius (Poir.) Greuter

Senecio squalidus L. subsp. microglossus (Guss.) Arcang.

Senecio squalidus L. subsp. rupestris (Waldst. \& Kit.) Greuter

E Senecio squalidus L. subsp. sardous (Fiori) Greuter

E Senecio squalidus $L$. subsp. squalidus

Senecio sylvaticus $L$.

E Senecio transiens (Rouy) Jeanm.

Senecio viscosus $L$.

T Senecio vulgaris L. subsp. denticulatus (O.F.Müll.) P.D.Sell

Senecio vulgaris $L$. subsp. vulgaris

Serratula tinctoria L. subsp. monticola (Boreau) Berher

Serratula tinctoria L. subsp. tinctoria

Silybum marianum (L.) Gaertn.

E Solidago litoralis Savi

Solidago virgaurea L. subsp. minuta (L.) Arcang.

Solidago virgaurea $L$. subsp. virgaurea

Sonchus arvensis $L$. subsp. arvensis

Sonchus arvensis L. subsp. uliginosus (M.Bieb.) Nyman

Sonchus asper (L.) Hill subsp. asper

Sonchus asper (L.) Hill subsp. glaucescens (Jord.) Ball

Sonchus bulbosus (L.) N.Kilian \& Greuter subsp. bulbosus

Sonchus maritimus $\mathrm{L}$.

Sonchus oleraceus $\mathrm{L}$.

Sonchus palustris L. - Note: Possibly extinct in Italy.

Sonchus tenerrimus $\mathrm{L}$.

Staehelina dubia L.

E Tanacetum audibertii (Req.) DC.

Tanacetum corymbosum (L.) Sch.Bip. subsp. achilleae (L.) Greuter

Tanacetum corymbosum (L.) Sch.Bip. subsp. corymbosum

Tanacetum corymbosum (L.) Sch.Bip. subsp. subcorymbosum (Schur) Pawl.

Tanacetum parthenium (L.) Sch.Bip.

ET Tanacetum vulgare L. subsp. siculum (Guss.) Raimondo \& Spadaro

Tanacetum vulgare $L$. subsp. vulgare

Taraxacum (Fontana) absurdum Soest

Taraxacum (Erythrosperma) acrocuspidatum Sonck

E Taraxacum (Alpestria) aemilianum Foggi \& Ricceri

Taraxacum (Alpestria) aequilobiforme Soest

Taraxacum (Alpestria) aestivum Soest

Taraxacum (Palustria) aginnense Hofstra

Taraxacum (Alpestria) albulense Soest

E Taraxacum (Palustria) amplexum Sonck 
Taraxacum (Taraxacum) ancistrolobum Dahlst.

Taraxacum (Palustria) annalisae Carlesi \& Peruzzi

Taraxacum (Alpina) apenninum (Ten.) DC.

Taraxacum (Erythrocarpa) apulicum Soest

Taraxacum (Erythrocarpa) aquilonare Hand.-Mazz.

Taraxacum (Palustria) arachnoideum Kirschner \& Štěpánek

Taraxacum (Fontana) aurantellum Soest

Taraxacum (Cucullata) aureocucullatum Soest

Taraxacum (Taraxacum) aurosuloides Soest

E Taraxacum (Erythrosperma) barbaricinum Arrigon

E Taraxacum (Taraxacum) bidentilobum Sonck

Taraxacum (Erythrosperma) botanicorum Sonck

Taraxacum (Erythrosperma) brachyglossum (Dahlst.) Raunk.

Taraxacum (Obliqua) caespitosum Soest

E Taraxacum (Erythrocarpa) calabricum Aquaro, Caparelli \& Peruzzi

Taraxacum (Palustria) candrianii Soest

Taraxacum (Erythrocarpa) capricum Soest

E Taraxacum (Scariosa) caramanicae Lojac.

E Taraxacum (Palustria) carthusianorum Aquaro, Caparelli \& Peruzzi

E Taraxacum (Erythrocarpa) cescae Aquaro, Caparelli \& Peruzzi

Taraxacum (Palustria) ciliare Soest

Taraxacum (Alpestria) cordatifolium Soest

Taraxacum (Fontana) croceicarpum Soest

Taraxacum (Alpestria) crocellum Soest

Taraxacum (Cucullata) cucullatum Dahlst.

Taraxacum (Taraxacum) debrayi Hagend., Soest \& Zevenb.

Taraxacum (Palustria) delanghei Soest

Taraxacum (Palustria) divulsifolium Soest

Taraxacum (Palustria) dolomiticum Soest

Taraxacum (Erythrosperma) dunensiforme Sonck

Taraxacum (Erythrosperma) edessicoides Uhlemann

DD Taraxacum (Taraxacum) ekmanii Dahlst.

Taraxacum (Erythrosperma) epirense Soest

Taraxacum (Erythrosperma) erythrospermum Andrz. ex Besser

Taraxacum (Taraxacum) fasciatiforme Soest

Taraxacum (Fontana) fontanicola Soest

Taraxacum (Fontana) fontanum Hand.-Mazz.

Taraxacum (Taraxacum) formosum Soest

Taraxacum (Palustria) frisicum Soest

Taraxacum (Erythrosperma) fulvum Raunk.

E Taraxacum (Erythrocarpa) garbarianum Peruzzi, Aquaro, Caparelli \& Raimondo

Taraxacum (Erythrosperma) gasparrinii Tineo ex Lojac.

E Taraxacum (Erythrosperma) genargenteum Arrigoni

Taraxacum (Taraxacum) gentile Rail.

ET Taraxacum (Taraxacum) gianninii Arrigoni, Ferretti \& Padula

E Taraxacum (Glacialia) glaciale É.Huet \& A. Huet ex Hand.-Mazz.

Taraxacum (Erythrosperma) gracillimum Soest

Taraxacum (Taraxacum) grossum Soest

Taraxacum (Arctica) handelii Murr

Taraxacum (Alpina) helveticum Soest

Taraxacum (Palustria) huterianum Soest

E Taraxacum (Erythrocarpa) kirschneri Aquaro, Caparelli \& Peruzzi

Taraxacum (Erythrosperma) lacistophylloides Dahlst.

Taraxacum (Erythrosperma) lacistophyllum (Dahlst.) Raunk.

E Taraxacum (Palustria) lacustre Soest

Taraxacum (Obliqua) lambinonii Soest

Taraxacum (Alpestria) Ianjouwii Soest

E Taraxacum (Palustria) lilianae Aquaro, Caparelli \& Peruzzi

Taraxacum (Palustria) limosicola Kirschner \& Štěpánek

Taraxacum (Taraxacum) linearisquameum Soest

ET Taraxacum (Erythrosperma) lucense Arrigoni, Ferretti \& Padula

Taraxacum (Taraxacum) lucescens Dahlst.

Taraxacum (Palustria) madidum Kirschner \& Štepánek - Note: Kirschner and Štepánek (1998) report a specimen from "Tyrol, Barda", very likely referring to Lago di Garda.

Taraxacum (Fontana) magnopyramidophorum Soest

E Taraxacum (Palustria) mannoccii Carlesi \& Peruzzi

Taraxacum (Alpestria) martellense Soest

Taraxacum (Alpina) mattmarkense Soest

Taraxacum (Scariosa) minimum (V.Brig.) N.Terracc.

Taraxacum (Erythrosperma) montesignum Soest

Taraxacum (Erythrosperma) multidentatum Soest

Taraxacum (Palustria) multisinuatum Kirschner, Sonck \& Štěpánek

Taraxacum (Celtica) nordstedtii Dahlst.

Taraxacum (Palustria) noterophilum Kirschner, Sonck \& Štěpánek

Taraxacum (Obovata) obovatum (Willd.) DC.

Taraxacum (Palustria) odiosum Kirschner \& Štěpánek

Taraxacum (Palustria) olivaceum Soest

E Taraxacum (Erythrocarpa) optimae Aquaro, Caparelli \& Peruzzi 
Taraxacum (Taraxacum) oreinicola Soest

Taraxacum (Alpina) oreophilum C.E.Haglund

Taraxacum (Borea) ostenfeldii Raunk.

Taraxacum (Alpina) pacheri Sch.Bip.

Taraxacum (Taraxacum) pallescentiforme Soest

DD Taraxacum (Alpestria) pallidisquameum Soest - Note: This species was recorded for Italy by Polatschek (1999).

Taraxacum (Alpina) panalpinum Soest

Taraxacum (Alpestria) parnassicum Dahlst.

Taraxacum (Palustria) pauckertianum Hudziok

Taraxacum (Taraxacum) pedemontanum Soest

Taraxacum (Alpina) pedrottii Soest

Taraxacum (Fontana) peralatum Soest

Taraxacum (Alpestria) perfissum Soest

Taraxacum (Erythrosperma) perincisum (Murr) Murr

E Taraxacum (Taraxacum) perrigidum Sonck

Taraxacum (Alpina) petiolulatum Huter

Taraxacum (Taraxacum) piceatum Dahlst.

Taraxacum (Taraxacum) piluliferum G.E.Haglund

Taraxacum (Erythrosperma) plumbeum Dahlst.

Taraxacum (Fontana) pohlii Soest

E Taraxacum (Erythrocarpa) pollinense Aquaro, Caparelli \& Peruzzi

Taraxacum (Alpestria) praeticum Soest

DD Taraxacum (Erythrosperma) proximum (Dahlst.) Raunk. - Note: This species is recorded from N and C Italy by Doll (1973), whitout precise localities.

Taraxacum (Taraxacum) pseudelongatum Soest

Taraxacum (Erythrosperma) pseudocastaneum Soest

Taraxacum (Alpina) pseudofontanum Soest

Taraxacum (Erythrocarpa) pseudohoppeanum Kirschner \& Štěpánek

Taraxacum (Palustria) refectum Sonck

Taraxacum (Arctica) reichenbachii (Huter) Dahlst.

Taraxacum (Alpestria) reophilum Soest

DD Taraxacum (Erythrosperma) retzii Soest - Note: This species is recorded from NW and C Italy by Doll (1973), without precise localities.

Taraxacum (Taraxacum) rhaeticum Soest

E Taraxacum (Taracaxum) rigidipes Sonck

Taraxacum (Taraxacum) rigidum Soest

DD Taraxacum (Erythrosperma) roseocarpum Soest - Note: This species is recorded from NW and C Italy (Soest 1969; Doll 1973), without precise localities. Taraxacum (Erythrosperma) rubicundum (Dahlst.) Dahlst.

Taraxacum (Fontana) rufocarpum Soest

Taraxacum (Taraxacum) sabaudum Soest

E Taraxacum (Erythrosperma) sarcidanum Arrigoni

ET Taraxacum (Erythrosperma) sardomontanum Arrigoni

Taraxacum (Erythrosperma) scanicum Dahlst.

Taraxacum (Palustria) scaturiginosum G.E.Haglund

Taraxacum (Rhodocarpa) schroeterianum Hand.-Mazz.

Taraxacum (Fontana) senile Soest

E Taraxacum (Palustria) siculum Soest

Taraxacum (Fontana) silvicola Soest

Taraxacum (Alpestria) simpliciusculum Soest

Taraxacum (Alpestria) stylosum Soest

Taraxacum (Erythrosperma) subdissimile Dahlst.

Taraxacum (Palustria) subolivaceum Sonck

Taraxacum (Taraxacum) subsaxenii Sahlin

Taraxacum (Erythrosperma) taraxacoides (Hoppe \& Hornsch.) Willk.

Taraxacum (Palustria) tenuifolium (Hoppe \& Hornsch.) W.D.J.Koch

Taraxacum (Cucullata) tiroliense Dahlst.

E Taraxacum (Alpina) tortilobiforme Soest

Taraxacum (Erythrosperma) tortilobum Florstr.

Taraxacum (Palustria) trilobifolium Hudziok

Taraxacum (Palustria) turfosum (Sch.Bip.) Soest

E Taraxacum (Erythrosperma) vaccarii Soest

Taraxacum (Erythrosperma) veglianum Uhlemann

Taraxacum (Taraxacum) valesiacum Soest

ET Taraxacum (Taraxacum) vallis-nibulae Arrigoni

Taraxacum (Alpina) venustum Dahlst.

Taraxacum (Alpina) vernelense Soest

Taraxacum (Alpina) vetteri Soest

Taraxacum (Palustria) vindobonense Soest

E Taraxacum (Erythrosperma) xantholigulatum Sonck

Taraxacum sect. Borealia Hand.-Mazz. - Note: The record of T. ceratophorum group for Udine province (FVG) by Aeschiman et al. (2014) is referred here.

Taraxacum F.H.Wigg. sect. Taraxacum - Note: In addition to the 25 apomictic taxa listed above (see also Kirschner et al. 2007 onwards), there are certainly

further, yet unidentified, taxa occurring in Italy, which are collectively referred here.

Tephroseris balbisiana (DC.) Holub

Tephroseris integrifolia (L.) Holub subsp. capitata (Wahlenb.) B.Nord.

Tephroseris integrifolia (L.) Holub subsp. integrifolia

E Tephroseris italica Holub

Tephroseris longifolia (Jacq.) Griseb. \& Schenk subsp. gaudinii (Gremli) Kerguélen

Tephroseris longifolia (Jacq.) Griseb. \& Schenk subsp. longifolia

Tephroseris longifolia (Jacq.) Griseb. \& Schenk subsp. pseudocrispa (Fiori) Greuter

Tolpis staticifolia (All.) Sch.Bip. 
Tolpis umbellata Bertol.

Tolpis virgata (Desf.) Bertol. subsp. grandiflora (Ten.) Arcang.

Tolpis virgata (Desf.) Bertol. subsp. virgata

Tragopogon crocifolius L. subsp. crocifolius

E Tragopogon crocifolius L. subsp. nebrodensis (Guss.) Raimondo

E Tragopogon cupanii Guss. ex DC.

Tragopogon dubius Scop.

Tragopogon eriospermus Ten.

Tragopogon minor Mill.

Tragopogon orientalis $\mathrm{L}$.

Tragopogon porrifolius $\mathrm{L}$.

Tragopogon pratensis $\mathrm{L}$.

Tragopogon samaritanii Heldr. \& Sartori ex Boiss.

Tragopogon tommasinii Sch.Bip.

Tripleurospermum inodorum (L.) Sch.Bip.

E Tripolium sorrentinoi (Tod.) Raimondo \& Greuter

Tussilago farfara $\mathrm{L}$.

Tyrimnus leucographus (L.) Cass.

Urospermum dalechampii (L.) F.W.Schmidt

Urospermum picroides (L.) Scop. ex F.W.Schmidt

Volutaria tubuliflora (Murb.) Sennen

Willemetia stipitata (Jacq.) Dalla Torre subsp. stipitata

Xanthium strumarium L. subsp. brasilicum (Vell.) O.Bolòs \& Vigo

Xanthium strumarium L. subsp. strumarium

Xeranthemum cylindraceum $\mathrm{Sm}$.

Xeranthemum inapertum (L.) Mill.

$E$

Xerolekia speciosissima (L.) Anderb.

Viburnaceae

E Adoxa moschatellina L. subsp. cescae Peruzzi \& N.G.Passal.

Adoxa moschatellina $\mathrm{L}$. subsp. moschatellina

Sambucus ebulus $L$.

Sambucus nigra $\mathrm{L}$.

Sambucus racemosa $L$. subsp. racemosa

Viburnum lantana $\mathrm{L}$.

Viburnum opulus $\mathrm{L}$.

Viburnum tinus L. subsp. tinus

Caprifoliaceae

Lonicera alpigena L. subsp. alpigena

Lonicera caerulea L. subsp. caerulea

Lonicera caprifolium $\mathrm{L}$.

Lonicera cyrenaica Viv.

Lonicera etrusca Santi

Lonicera implexa Aiton subsp. implexa

Lonicera nigra $\mathrm{L}$.

Lonicera periclymenum $\mathrm{L}$.

$E$

Lonicera stabiana Guss. ex Pasq.

Lonicera xylosteum L. - Note: Lonicera xylosteum L. var. nigra Loisel. was recorded for central and southern Italy (Conti et al. 2005).

Linnaeaceae

Linnaea borealis L.

Dipsacaceae

Cephalaria alpina (L.) Roem. \& Schult.

Cephalaria joppensis (Rchb.) Coult.

Cephalaria leucantha (L.) Roem. \& Schult.

E Cephalaria mediterranea (Viv.) Szabó

E Cephalaria squamiflora (Sieber) Greuter subsp. bigazzii (Bacch., Brullo \& Giusso) Domina

Cephalaria transsylvanica (L.) Roem. \& Schult.

E Dipsacus ferox Loisel.

Dipsacus fullonum $L$. subsp. fullonum

Dipsacus pilosus $\mathrm{L}$.

E Dipsacus valsecchiae Camarda

Knautia arvensis (L.) Coult.

E Knautia baldensis A.Kern. ex Borbás

E Knautia calycina (C.Presl) Guss.

Knautia collina Jord.

E Knautia dinarica (Murb.) Borbás subsp. silana (Grande) Ehrend. - Note: For the taxonomy and distribution of this unit, see Peruzzi et al. (2013a).

T Knautia drymeja Heuff. subsp. centrifrons (Borbás) Ehrend. - Note: Recently, K. drymeja was regarded as a morphologically and genetically variable species without infraspecific taxa, also including $K$. gussonei (Rešetnik et al. 2016). The authors based their morphometric analyses on single specimens for 43 populations of the complex. Only a single voucher specimen of $K$. gussonei was studied. Furtermore, some characters useful for identification in morphometric analyses (i.e., presence of glandular hairs, see Bartolucci and Conti 2014) were not considered at all. Waiting for further studies, we are provisionally maintaining here the taxonomic treatment proposed by Conti et al. (2005).

Knautia drymeja Heuff. subsp. drymeja 
T Knautia drymeja Heuff. subsp. tergestina (Beck) Ehrend.

Knautia fleischmannii (Hladnik ex Rchb.) Pacher - Note: Possibly extinct in Italy.

ET Knautia gussonei Szabó

Knautia illyrica Beck

Knautia integrifolia (L.) Bertol. subsp. integrifolia

Knautia longifolia (Waldst. \& Kit.) W.D.J.Koch

E

Knautia lucana Lacaita \& Szabó

Knautia maxima (Opiz) J.Ortmann subsp. maxima - Note: The record of K. maxima from MOL (Lucchese 1996; under the name K. dipsacifolia) probably refers to K. gussonei Szabó.

Knautia maxima (Opiz) J.Ortmann subsp. sixtina (Briq.) Bartolucci \& Galasso

Knautia mollis Jord.

E Knautia persicina A.Kern.

Knautia ressmannii (Pacher) Briq.

Knautia subcanescens Jord.

Knautia transalpina (Christ) Briq.

Knautia velutina Briq.

Lomelosia argentea (L.) Greuter \& Burdet

Lomelosia brachiata $(\mathrm{Sm}$.) Greuter \& Burdet

Lomelosia crenata (Cirillo) Greuter \& Burdet subsp. crenata

T Lomelosia crenata (Cirillo) Greuter \& Burdet subsp. dallaportae (Boiss.) Greuter \& Burdet

ET Lomelosia crenata (Cirillo) Greuter \& Burdet subsp. pseudisetensis (Lacaita) Greuter \& Burdet

Lomelosia cretica (L.) Greuter \& Burdet

Lomelosia divaricata (Jacq.) Greuter \& Burdet

Lomelosia graminifolia (L.) Greuter \& Burdet subsp. graminifolia

Lomelosia rutifolia (Vahl) Avino \& P.Caputo

Lomelosia simplex (Desf.) Raf. subsp. simplex

Lomelosia stellata (L.) Raf.

E Pseudoscabiosa limonifolia (Vahl) Devesa

Scabiosa columbaria L. subsp. columbaria

Scabiosa columbaria L. subsp. portae (A.Kern. ex Huter) Hayek

E Scabiosa holosericea Bertol.

Scabiosa lucida Vill. subsp. Iucida

Scabiosa mollissima Viv.

Scabiosa ochroleuca L.

E Scabiosa parviflora Desf.

Scabiosa pyrenaica All.

Scabiosa silenifolia Waldst. \& Kit.

Scabiosa taygetea Boiss. \& Heldr. subsp. garganica (Porta \& Rigo) Hayek

Scabiosa triandra L.

ET Scabiosa uniseta Savi

Scabiosa velenovskyana Bobrov

E Scabiosa vestina Facchini ex W.D.J.Koch

Sixalix atropurpurea (L.) Greuter \& Burdet

Succisa pratensis Moench

Succisella inflexa (Kluk) Beck

Valerianaceae

Taxonomic references: Valeriana officinalis L. group (Kirschner and Raab-Straube 2017).

E Centranthus amazonum Fridl. \& A.Raynal

Centranthus angustifolius (Mill.) DC. subsp. angustifolius

Centranthus calcitrapae (L.) Dufr. subsp. calcitrapae

Centranthus ruber (L.) DC. subsp. ruber

Fedia graciliflora Fisch. \& C.A.Mey.

Valeriana celtica L. subsp. celtica

Valeriana celtica L. subsp. norica Vierh.

Valeriana dioica L.

Valeriana elongata Jacq.

Valeriana excelsa Poir. subsp. sambucifolia (J.C.Mikan ex Pohl) Holub

Valeriana excelsa Poir. subsp. versifolia (Brügger) Buttler, Hand \& Kirschner

Valeriana montana L.

Valeriana officinalis L. subsp. nemorensis (B.Turk) F.Martini \& Soldano

Valeriana officinalis L. subsp. officinalis

Valeriana saliunca All.

Valeriana saxatilis $\mathrm{L}$.

Valeriana stolonifera Czern. subsp. angustifolia Soó

Valeriana supina Ard.

Valeriana tripteris L. subsp. austriaca E.Walther

Valeriana tripteris L. subsp. tomentella E.Walther

Valeriana tripteris L. subsp. tripteris

Valeriana tuberosa $\mathrm{L}$.

Valerianella carinata Loisel.

Valerianella coronata (L.) DC.

Valerianella costata (Stev.) Betcke

Valerianella dentata (L.) Pollich

Valerianella discoidea (L.) Loisel.

Valerianella echinata (L.) DC.

Valerianella eriocarpa Desv. 
Valerianella locusta (L.) Laterr.

Valerianella microcarpa Loisel.

Valerianella muricata (Stev. ex M.Bieb.) J.W.Loudon

Valerianella puberula (Bertol. ex Guss.) DC.

Valerianella pumila (L.) DC.

Valerianella rimosa Bastard

Valerianella turgida (Stev.) Betcke

Valerianella vesicaria (L.) Moench

Araliaceae

Hedera helix L. subsp. helix

Hydrocotyle vulgaris L.

Apiaceae

Taxonomic references: Laserpitium L., Siler Mill., Silphiodaucus (Koso-Pol.) Spalik, Wojew., Banasiak, Piwczyński \& Reduron and Thapsia L. (Banasiak et al. 2016; lamonico et al. 2016a).

Aegopodium podagraria $\mathrm{L}$.

Aethusa cynapium L. subsp. cynapium

Aethusa cynapium L. subsp. elata (Friedl.) Schübl. \& G.Martens

Ammi majus L.

Ammoides pusilla (Brot.) Breistr.

Angelica sylvestris L. subsp. sylvestris

Anthriscus caucalis M.Bieb.

Anthriscus nemorosa (M.Bieb.) Spreng.

Anthriscus nitida (Wahlenb.) Hazsl.

Anthriscus sylvestris (L.) Hoffm. subsp. alpina (Vill.) Gremli

Anthriscus sylvestris (L.) Hoffm. subsp. sylvestris

Apium graveolens $\mathrm{L}$.

Astrantia bavarica F.W.Schultz

Astrantia carniolica Jacq.

Astrantia major L. subsp. involucrata (W.D.J.Koch) Ces.

Astrantia major L. subsp. major

Astrantia minor L.

E Astrantia pauciflora Bertol. subsp. pauciflora

E Astrantia pauciflora Bertol. subsp. tenorei (Mariotti) Bechi \& Garbari

E Athamanta cortiana Ferrarini

Athamanta cretensis L.

Athamanta ramosissima Port.

Athamanta sicula $\mathrm{L}$.

Athamanta turbith (L.) Brot. subsp. turbith

Berula erecta (Huds.) Coville

Bifora radians M.Bieb.

Bifora testiculata (L.) Spreng.

Bonannia graeca (L.) Halácsy

Bubon macedonicum $\mathrm{L}$.

Bunium bulbocastanum $\mathrm{L}$.

E Bunium corydalinum DC

Bunium macuca Boiss.

E Bunium petraeum Ten.

Bupleurum affine Sadler

Bupleurum baldense Turra

E Bupleurum dianthifolium Guss.

E Bupleurum elatum Guss.

Bupleurum falcatum L. subsp. cernuum (Ten.) Arcang.

E Bupleurum falcatum L. subsp. corsicum (Coss. \& Kralik) Rouy \& E.G.Camus

Bupleurum fruticosum $\mathrm{L}$.

Bupleurum gerardi All.

Bupleurum gracile d'Urv.

E Bupleurum gussonei (Arcang.) Snogerup \& B.Snogerup

Bupleurum Iancifolium Hornem.

Bupleurum longifolium L.

Bupleurum odontites $\mathrm{L}$.

Bupleurum petraeum $\mathrm{L}$.

Bupleurum praealtum $\mathrm{L}$.

Bupleurum ranunculoides L. subsp. ranunculoides

Bupleurum ranunculoides L. subsp. telonense (Gren. ex Timb.-Lagr.) Coste

Bupleurum rigidum $\mathrm{L}$. subsp. rigidum

E Bupleurum rollii (Montel.) Moraldo - For the taxonomy and distribution of this species and related taxa, see Iberite et al. (2015).

Bupleurum semicompositum $\mathrm{L}$.

Bupleurum stellatum L.

Bupleurum subovatum Link ex Spreng.

Bupleurum tenuissimum $\mathrm{L}$.

C Bupleurum trichopodum Boiss. \& Spruner

Bupleurum veronense Turra

Cachrys cristata DC.

Cachrys libanotis L.

Cachrys pungens Jan ex Guss. 
Sicula $\mathrm{L}$.

Carum appuanum (Viv.) Grande subsp. appuanum

Carum carvi $\mathrm{L}$.

Carum heldreichii Boiss.

Caucalis platycarpos $\mathrm{L}$.

Cervaria rivini Gaertn.

Chaerophyllum aromaticum $\mathrm{L}$.

Chaerophyllum aureum $\mathrm{L}$.

Chaerophyllum elegans Gaudin

Chaerophyllum hirsutum $\mathrm{L}$.

E Chaerophyllum magellense Ten.

Chaerophyllum nodosum (L.) Crantz

Chaerophyllum temulum $\mathrm{L}$.

Chaerophyllum villarsii W.D.J.Koch

Cicuta virosa $\mathrm{L}$.

Conium maculatum L. subsp. maculatum

Conopodium majus (Gouan) Loret subsp. majus

E

Coristospermum cuneifolium (Guss.) Bertol.

Coristospermum ferulaceum (All.) Reduron, Charpin \& Pimenov

Coristospermum seguieri (Jacq.) Banfi, Galasso \& Soldano

Crithmum maritimum $\mathrm{L}$.

E Cryptotaenia thomasii (Ten.) DC

Daucus aureus Desf.

E Daucus broteroi Ten

Daucus carota L. subsp. carota

Daucus carota L. subsp. commutatus (Paol.) Thell.

Daucus carota L. subsp. drepanensis (Tod. ex Lojac.) Heywood

Daucus carota L. subsp. gummifer (Syme) Hook.f.

Daucus carota L. subsp. hispanicus (Gouan) Thell.

Daucus carota L. subsp. maritimus (Lam.) Batt.

Daucus carota L. subsp. maximus (Desf.) Ball

ET Daucus carota L. subsp. rupestris (Guss.) Heywood

Daucus guttatus Sm.

Daucus minusculus Pau ex Font Quer

Daucus muricatus (L.) L.

E Daucus nebrodensis Strobl

Daucus pumilus (L.) Hoffmanns. \& Link

Daucus rouyi Spalik \& Reduron

Dichoropetalum carvifolium-chabraei (Crantz) Soldano, Galasso \& Banfi

Dichoropetalum schottii (Bess. ex DC.) Pimenov \& Kljuykov

Echinophora spinosa $\mathrm{L}$.

Echinophora tenuifolia L. subsp. tenuifolia

Eryngium alpinum $\mathrm{L}$.

Eryngium amethystinum $\mathrm{L}$.

Eryngium campestre $\mathrm{L}$.

Eryngium corniculatum Lam.

E Eryngium crinitum C.Presl

Eryngium dichotomum Desf.

Eryngium maritimum $\mathrm{L}$.

Eryngium pusillum $\mathrm{L}$.

E Eryngium siculum Lojac.

Eryngium spinalba Vill.

E Eryngium tricuspidatum L. subsp. bocconei (Lam.) Wörz

Eryngium tricuspidatum $\mathrm{L}$. subsp. tricuspidatum

Eryngium triquetrum Vah

Falcaria vulgaris Bernh.

E Ferula arrigonii Bocchieri

Ferula communis $L$. subsp. communis

Ferula glauca $\mathrm{L}$.

Ferulago campestris (Besser) Grecescu

E Ferulago nodosa (L.) Boiss. subsp. geniculata (Guss.) Troìa \& Raimondo - Note: For the nomenclature of this subspecies, see Peruzzi et al. (2015b). Ferulago sylvatica (Besser) Rchb.

T Foeniculum vulgare Mill. subsp. piperitum (Ucria) Bég.

Foeniculum vulgare Mill. subsp. vulgare

Geocaryum capillifolium (Guss.) Coss.

Geocaryum cynapioides (Guss.) Engstrand

Grafia golaka (Hacq.) Rchb.

Hacquetia epipactis (Scop.) DC.

Hellenocarum multiflorum (Sm.) H.Wolff

Helosciadium crassipes W.D.J.Koch ex Rchb.

Helosciadium inundatum (L.) W.D.J.Koch

Helosciadium nodiflorum (L.) W.D.J.Koch subsp. nodiflorum

Helosciadium repens (Jacq.) W.D.J.Koch - Note: Possibly extinct in Italy (Rossi et al. 2013).

Heptaptera angustifolia (Bertol.) Tutin

Heracleum austriacum $\mathrm{L}$.

Heracleum orsinii Guss.

Heracleum pyrenaicum Lam. subsp. pollinianum (Bertol.) F.Pedrotti \& Pignatti

Heracleum sibiricum L. subsp. sibiricum 
Heracleum sibiricum L. subsp. ternatum (Velen.) Briq.

Heracleum sphondylium L. subsp. elegans (Crantz) Schübl. \& G.Martens

Heracleum sphondylium L. subsp. sphondylium

Imperatoria ostruthium $\mathrm{L}$.

Katapsuxis silaifolia (Jacq.) Reduron, Charpin \& Pimenov

Krubera peregrina (L.) Hoffm.

Kundmannia sicula (L.) DC

Lagoecia cuminoides L.

Laserpitium gallicum L. subsp. gallicum

Laserpitium halleri Crantz subsp. halleri

Laserpitium krapfii Crantz subsp. gaudinii (Moretti) Thell.

Laserpitium Iatifolium L.

Laserpitium nestleri Soy.-Will.

Laserpitium nitidum Zanted.

Laserpitium peucedanoides $\mathrm{L}$.

Magydaris pastinacea (Lam.) Paol.

Meum athamanticum Jacq.

Molopospermum peloponnesiacum (L.) W.D.J.Koch subsp. bauhinii I.Ullmann

Molopospermum peloponnesiacum (L.) W.D.J.Koch subsp. peloponnesiacum

Mutellina adonidifolia (J.Gay) Gutermann

Myrrhis odorata (L.) Scop.

Oenanthe aquatica (L.) Poir.

Oenanthe crocata $\mathrm{L}$.

Oenanthe fistulosa $\mathrm{L}$.

Oenanthe globulosa $\mathrm{L}$.

Oenanthe lachenalii C.C.Gmel.

Oenanthe lisae Moris

Oenanthe peucedanifolia Pollich

Oenanthe pimpinelloides $\mathrm{L}$.

Oenanthe silaifolia M.Bieb.

Opopanax chironium (L.) W.D.J.Koch

Opopanax hispidus (Friv.) Griseb.

Oreoselinum nigrum Delarbre

Orlaya daucorlaya Murb.

Orlaya grandiflora (L.) Hoffm.

Orlaya platycarpos W.D.J.Koch

Pachypleurum mutellinoides (Crantz) Holub

Pastinaca kochii Duby

Pastinaca sativa $L$. subsp. sativa

Pastinaca sativa L. subsp. urens (Req. ex Godr.) Čelak.

E Petagnaea gussonei (Spreng.) Rauschert

Peucedanum coriaceum Rchb.

Peucedanum officinale $L$. subsp. officinale

C Peucedanum paniculatum Loisel. - Note: This species is considered as a narrow endemic to Corse by Tison and de Foucault (2014). In addition, Arrigoni (2013) recorded it as doubtfully native in SAR.

Physospermum cornubiense (L.) DC.

Physospermum verticillatum (Waldst. \& Kit.) Vis.

E Pimpinella anisoides V.Brig.

Pimpinella gussonei Bertol.

Pimpinella lutea Desf.

Pimpinella major (L.) Huds.

Pimpinella peregrina $\mathrm{L}$.

Pimpinella saxifraga L. subsp. alpina Nyman

Pimpinella saxifraga L. subsp. nigra (Mill.) Ces.

Pimpinella saxifraga $\mathrm{L}$. subsp. saxifraga

Pimpinella tragium Vill.

Pleurospermum austriacum (L.) Hoffm.

Prangos ferulacea (L.) Lindl.

Prangos trifida Herrnst. \& Heyn

Pteroselinum austriacum (Jacq.) Rchb.

E Ptychotis sardoa Pignatti \& Metlesics

Ptychotis saxifraga (L.) Loret \& Barrandon

Ridolfia segetum (Guss.) Moris

Sanicula europaea L.

Scandix australis L. subsp. australis

Scandix australis L. subsp. grandiflora (L.) Thell.

Scandix macrorhyncha C.A.Mey.

Scandix pecten-veneris L. subsp. brachycarpa (Guss.) Thell.

Scandix pecten-veneris $L$. subsp. pecten-veneris

Selinum carvifolia (L.) L.

Seseli annuum L. subsp. annuum

Seseli annuum L. subsp. carvifolium (Vill.) P.Fourn.

Seseli austriacum (G.Beck) Wohlf.

E Seseli bocconei Guss.

Seseli galloprovinciale Reduron

Seseli kochii Breistr.

Seseli libanotis (L.) W.D.J.Koch

Seseli montanum L. subsp. montanum 
Seseli pallasii Besser

Seseli peucedanoides (M.Bieb.) Koso-Pol.

E Seselipolyphyllum Ten.

Seseli praecox (Gamisans) Gamisans

Seseli tommasinii Rchb.f.

ET Seseli tortuosum L. subsp. maritimum (Guss.) C.Brullo, Brullo, Giusso \& Sciandr.

Seseli tortuosum L. subsp. tortuosum

E Siculosciadium nebrodense (Guss.) C.Brullo, Brullo, S.R.Downie \& Giusso

Silaum silaus (L.) Schinz \& Thell.

Siler montanum Crantz subsp. garganicum (Ten.) lamonico, Bartolucci \& F.Conti

Siler montanum Crantz subsp. montanum

E Siler montanum Crantz subsp. siculum (Spreng.) lamonico, Bartolucci \& F.Conti Silphiodaucus prutenicus (L.) Spalik, Wojew., Banasiak, Piwczyński \& Reduron

Sison amomum L.

Sison segetum $\mathrm{L}$.

Sium Iatifolium L.

Smyrnium olusatrum $\mathrm{L}$.

T Smyrnium perfoliatum L. subsp. dimartinoi (Raimondo, Mazzola \& Spadaro) Stinca \& Pignatti

Smyrnium perfoliatum L. subsp. perfoliatum

Smyrnium perfoliatum L. subsp. rotundifolium (Mill.) Bonnier \& Layens

Thapsia asclepium L.

Thapsia garganica L. subsp. garganica

E Thapsia garganica L. subsp. messanensis (Guss.) Brullo, Guglielmo, Pasta, Pavone \& Salmeri

Thapsia meoides (Desf.) Guss.

E Thapsia pelagica Brullo, Guglielmo, Pasta, Pavone \& Salmeri

Thysselinum palustre (L.) Hoffm.

Tommasinia altissima (Mill.) Reduron

Tordylium apulum $\mathrm{L}$.

Tordylium maximum $\mathrm{L}$.

Tordylium officinale L.

Torilis africana Spreng.

Torilis arvensis (Huds.) Link subsp. arvensis

Torilis arvensis (Huds.) Link subsp. neglecta Thell.

Torilis elongata (Hoffmanns. \& Link) Samp.

Torilis japonica (Houtt.) DC.

Torilis leptophylla (L.) Rchb.f.

E Torilis nemoralis (Brullo) Brullo \& Giusso

Torilis nodosa (L.) Gaertn. subsp. nodosa

Torilis nodosa (L.) Gaertn. subsp. webbii (Jury) Kerguélen

Trinia dalechampii (Ten.) Janch.

Trinia glauca (L.) Dumort. subsp. carniolica (A.Kern. ex Janch.) H.Wolff

Trinia glauca (L.) Dumort. subsp. glauca

Trochiscanthes nodiflora (All.) W.D.J.Koch

Turgenia latifolia (L.) Hoffm.

E Visnaga crinita (Guss.) Giardina \& Raimondo - Note: Possibly extinct in CAL (Peruzzi 2014).

Visnaga daucoides Gaertn.

Xanthoselinum venetum (Spreng.) Soldano \& Banfi

\section{Discussion}

The native flora of Italy include 8195 specific and subspecific taxa. It is the highest number in Europe and, at the Mediterranean region level, only Turkey hosts a higher number of native plant taxa (Boulos 1995; Güner et al. 2000; Valdés et al. 2002; Lauber and Wagner 2007; Buttler and Hand 2008; Danihelka et al. 2012; Güner et al. 2012; Aedo et al. 2013; Dimopoulos et al. 2013; Tison and de Foucault 2014; Vangjeli 2015; Dimopoulos et al. 2016; Raab-Straube et al. 2016; Aedo et al. 2017; Özhatay et al. 2017). The Italian endemics (1708) correspond to $20,8 \%$ of the total flora. The degree of endemism for Italy is comparable with most of the European and Mediterranean floras, with the exception of the Turkish flora, in which $34 \%$ of endemicity is reported (Aedo et al. 2013; Özhatay et al. 2013; Rankou et al. 2013). Recently, Peruzzi et al. (2014a) reported for Italy 1371 endemic taxa, then updated to 1400 by Peruzzi et al. (2015b), but these authors deliberately excluded the numerous subspecies within the taxonomically critical genera Hieracium and Pilosella.

Although in recent years field explorations, regional floras and taxonomic reviews regarding the Italian flora have been increasingly published, for many taxa the distribution data are lacking and/or show significant gaps of knowledge. In fact, 568 taxa have not been confirmed in recent times, 99 are doubtfully occurring in Italy, 19 are data deficient and 430 are taxonomically doubtful (not to mention hundreds of Hieracium subspecies). There is clearly a need for further field and biosystematic investigations to fill these gaps. Accordingly, the number of taxa reported here is certainly not final and the taxonomic circumscription of some critical genera will also require further in-depth analyses. In particular, taxonomic studies should pay a special attention to the correct interpretation and management of the taxonomic rank of subspecies (Peruzzi and Bartolucci 2016). For instance, there are several cases in the mostly apomictic genus Hieracium, where field observation suggests the co-occurrence in the same place of more than one putative "subspecies". Similar situations could apply to many other taxa where "subspecies"lack a clear geographical (or at least ecological) structure. The classical subspecies concept provided by Wilson and Brown (1953), i.e. "the subspecies were conceived of as genetically distinct, geographically isolated population belonging to the same species and therefore interbreeding freely at the zones of contact", is difficult to apply when two or more subspecies share the same native 
range, show geographical and ecological overlap or are not sexually reproducing. These problems will need further research of experimental taxonomy and more consistency in using the subspecies rank among taxonomists (Hamilton and Reichard 1992).

Despite these problems, an updated checklist is a fundamental tool in floristic, taxonomic and particularly in conservation research (Lomolino 2004; Whittaker et al. 2005).

\section{Acknowledgements}

We gratefully acknowledge collegues and friends who provided distribution, nomenclatural and taxonomic advices: Michele Adorni, Aldo Antonietti, Loris Bagli, Fausto Bonafede, Giovanna Bosi, Eugenia Bugni, Mario Calbi, Antonio Croce, Roberto Dellavedova, Marco D'Antraccoli, Giorgio Faggi, Germano Federici, Umberto Ferrando, Giulio Ferretti, Filiberto Fiandri, Helmut Freitag, Bruno Gallino, Giovanni Gestri, Luigi Ghillani, Matteo Gualmini, Laura Guglielmone, Sigurd E. Fröhner, Cesare Lasen, Wolfgang Licht, Umberto Lodesani, Fabrizio Martini, Marta Bandini Mazzanti, Anna Maria Mercuri, Nicola Merloni, Sergio Montanari, Villiam Morelli, Sergei L. Mosyakin, Franco Orsino, Konrad Pagitz, Guglielmo Pandolfo, Roberto Pascalt, Marziano Pascale, Mauro Pellizzari, Enrico V. Perrino, Franco Picco, Filippo Piccoli, Annalaura Pistarino, Enrico Romani, Daniele Saiani, Claudio Santini, Fabio Semprini, Consolata Siniscalco, Giancarlo Tondi and Michele Vignodelli.

\section{Disclosure statement}

No potential conflict of interest was reported by the authors.

\section{ORCID}

F. Bartolucci (iD) http://orcid.org/0000-0001-8199-6003

L. Peruzzi (D) http://orcid.org/0000-0001-9008-273X

G. Galasso (D) http://orcid.org/0000-0002-2501-456X

A. Albano (iD http://orcid.org/0000-0002-0874-320X

N. M. G. Ardenghi iD http://orcid.org/0000-0002-4366-5729

G. Astuti (iD http://orcid.org/0000-0001-5790-3516

G. Bacchetta (iD http://orcid.org/0000-0002-1714-3978

L. Cecchi (iD http://orcid.org/0000-0001-9706-0947

G. Domina (iD) http://orcid.org/0000-0003-4184-398X

G. Fenu (iD http://orcid.org/0000-0003-4762-5043

B. Foggi iD http://orcid.org/0000-0001-6451-4025

D. lamonico (iD http://orcid.org/0000-0001-5491-7568

E. Martinetto (iD http://orcid.org/0000-0002-9131-0877

R. Pennesi (iD http://orcid.org/0000-0002-6145-5581

F. Roma-Marzio (iD http://orcid.org/0000-0002-2184-0311

A. Santangelo (iD http://orcid.org/0000-0001-5560-1725

A. Scoppola iD http://orcid.org/0000-0001-7389-5608

F. Selvi (iD http://orcid.org/0000-0002-3820-125X

A. Stinca (D) http://orcid.org/0000-0002-8275-0184

R. P. Wagensommer (iD http://orcid.org/0000-0002-1614-4821

F. Conti (D) http://orcid.org/0000-0001-7391-6691

\section{References}

Accorsi CA, Bandini Mazzanti M, Forlani L, Meneghel M, Rigoni A, Sorbini L. 1989. Primi dati stratigrafici e palinologici sulla sequenza di Bernascone (Verona), datata alla base $18.870 \pm 300$ B.P. Inform Bot Ital. 21: 240-245.

Aedo C, Buira A, Medina L, Fernández-Albert M. 2017. The Iberian vascular flora richness, endemicity and distribution patterns. In: Loidi J, editor. The vegetation of the Iberian Peninsula. Vol. 1, Cham: Springer. p. 101-130. doi: 10.1007/978-3-319-54784-8.

Aedo C, Medina L, Fernández-Albert M. 2013. Species richness and endemicity in the Spanish vascular flora. Nord J Bot. 31: 478-488. doi:10.1111/j.1756-1051.2012.00009.x.

Aeschimann D, Lauber K, Moser DM, Theurillat J-P. 2004. Flora alpina, Vol. 2. Bern: Haupt Verlag.
Akhani H, Edwards G, Roalson EH. 2007. Diversification of the old world Salsoleae s.l. (Chenopodiaceae): molecular phylogenetic analysis of nuclear and chloroplast data sets and a revised classification. Int J Plant Sci. 168: 931-956. doi:10.1086/518263.

Alessandrini A, Buono V, Longo D, Magni C, Manni QG, Nicolella G, editors. 2017. Acta Plantarum Notes 5, Le raccolte di Acta Plantarum. Boves (Cuneo): Araba Fenice.

Al-ShehbazIA, German DA, MummenhoffK, Moazzeni H. 2014. Systematics, tribal placements, and synopses of the Malcolmia s.l. segregates (Brassicaceae). Harvard Pap Bot. 19:53-71. doi:10.3100/hpib.v19iss1.2014.n4.

APG IV. 2016. An update of the Angiosperm Phylogeny Group classification for the orders and families of flowering plants: APG IV. Bot J Linn Soc. 181: 1-20. doi:10.1111/j.1095-8339.2009.00996.x.

Aquaro G, Caparelli KF, Peruzzi L. 2008. The genus Taraxacum (Asteraceae) in Italy. I. A systematic study of Taraxacum sect. Palustria. Phytol Balcan. 14: 61-67.

Aquaro G, Caparelli KF, Peruzzi L. 2009. The genus Taraxacum (Asteraceae) in Italy. II. Five new species of Taraxacum sect. Erythrocarpa. In: Ivanova D, editor. Plant, fungal and habitat diversity investigation and conservation. Proceedings of IV Balcan Botanical Congress, Sofia, June 20-26, 2006. Sofia: Institute of Botany.p. 160-168.

Ardenghi NMG, Foggi B. 2015. Lectotypification and combination of Festuca apennina (Poaceae). Taxon. 64: 1038-1041. doi:10.12705/645.14.

Arrigoni PV. 2006. Flora dell'Isola di Sardegna 1 [Flora of Sardinia 1]. Sassari: Carlo Delfino Editore. Italian.

Arrigoni PV. 2007. Contributo alla conoscenza della flora della Sardegna: nuove specie di Taraxacum e altri reperti [Contribution to the knowledge of the flora of Sardinia: new species of Taraxacum and other specimens]. Parlatorea. 9: 87-94. Italian.

Arrigoni PV. 2012. Miscellaneous notes about some taxa of the Italian flora. Webbia. 67: 37-46. doi:10.1080/00837792.2012.10670907.

Arrigoni PV, Ferretti G, Padula M. 2006. Due nuove specie di Taraxacum dell'Appennino Lucchese (Toscana settentrionale) [Two new species of Taraxacum of the Lucchese Apennine (Northern Tuscany)]. Parlatorea. 8: 5-9. Italian.

Arrigoni PV. 2013. Flora dell'Isola di Sardegna 4 [Flora of Sardinia 4]. Sassari: Carlo Delfino Editore. Italian.

Arrigoni PV. 2014. Revisione tassonomica e corologica del genere Polygala in Italia [Taxonomic revision of the genus Polygala in Italy]. Inform Bot Ital. 46: 235-263. Italian.

Arrigoni PV. 2015. Contribution to the study of the genus Armeria (Plumbaginaceae) in the Italian peninsula. Flora Medit. 25(Special issue): 7-32.

Astuti G, Bedini G, Carta A, Roma-Marzio F, Trinco A, Peruzzi L. 2018. Comparative assessment of reproductive traits across different habitats in the endangered Webb's hyacinth (Bellevalia webbiana Parl.). Nat Cons. 24: 81-92. doi:10.3897/natureconservation.24.20650.

Astuti G, Brullo S, Domina G, El Mokni R, Giordani T, Peruzzi L. 2017a. Phylogenetic relationships among tetraploid species of Bellevalia (Asparagaceae) endemic to South-Central Mediterranean. Plant Biosyst. 151: 1120-1128. doi:10.1080/11263504.2017.1320308.

Astuti G, Cristofolini G, Peruzzi L, Pupillo P. 2014. A new subspecies of Pulmonaria officinalis (Boraginaceae) from the Southern Alps. Phytotaxa. 186: 148-157. doi:10.11646/phytotaxa.186.3.3.

Astuti G, Peruzzi L. 2018. Are shoots of diagnostic value in Central European bladderworts (Utricularia L, Lentibulariaceae)? Plant Biosyst.

Astuti G, Roma-Marzio F, D'Antraccoli M, Bedini G, Carta A, Sebastiani F, Bruschi P, Peruzzi L. 2017b. Conservation biology of the last Italian population of Cistus laurifolius (Cistaceae): demographic structure, genetics and reproductive success. Nat Cons. 22: 169-190. doi:10.3897/ natureconservation.22.19809.

Astuti G, Roma-Marzio F, Peruzzi L. 2015. The genus Picris (Asteraceae) in southern Italy: contribution to its systematic knowledge. Phytotaxa. 207: 106-114. doi:10.11646/phytotaxa.207.1.5.

Bacchetta G, Brullo S, Congiu A, Fenu G, Garrido JL, Mattana E. 2012a. A new species of Aquilegia (Ranunculaceae) from Sardinia (Italy). Phytotaxa. 56: 59-64. doi:10.11646/phytotaxa.56.1.9.

Bacchetta G, Brullo S, D'Emerico S, Pontecorvo C, Salmeri C. 2012b. Charybdis glaucophylla (Asparagaceae), a new species from Sardinia. Phytotaxa. 69: 16-26. doi:10.11646/phytotaxa.69.1.4. 
Bacchetta G, Carta A, Paradis G, Piazza C, Peruzzi L. 2014. Further insights into the taxonomy of the Silene nocturna species complex (Caryophyllaceae): a systematic survey of the taxa from Sardinia and Corsica. Phytotaxa. 175: 37-44. doi:10.11646/phytotaxa.175.1.4.

Bagella S, Peruzzi L, Caria MC, Filigheddu R. 2015. Unraveling the taxonomy and nomenclature Isoetes histrix Bory species complex (Isoetaceae, Lycopodiidae). Turk J Bot. 39: 383-387. doi:10.3906/bot-1404-121.

Banasiak Ł, Wojewódzka A, Baczyński J, Reduron JP, Piwczyński M, KurzynaMłynik R, Gutaker R, Czarnocka-Cieciura A, Kosmala-Grzechnik S, Spalik K. 2016. Phylogeny of Apiaceae subtribe Daucinae and the taxonomic delineation of its genera. Taxon. 65: 563-585. doi:10.12705/653.8.

Banfi E. 2000. Diversità tassonomica ed eco-corologica alle alte quote in Italia: il caso delle Poaceae. Ann Mus Civici-Rovereto. 14(1998): 5-30.

Banfi E. 2017. Helictochloa Romero Zarco. In: Pignatti S, editor. Flora d'Italia 1 [Flora of Italy 1]. Edagricole: Milano. pp. 633-636. Italian.

Banfi E, Galasso G, Bartolucci F. 2018. Nomenclatural novelties for the Euro+Med flora. Nat Hist Sci. doi:10.4081/nhs.2018.365.

Banfi E, Galasso G, Foggi B, Kopecký D, Ardenghi NMG. 2017. From Schedonorus and Micropyropsis to Lolium (Poaceae: Loliinae): new combinations and typifications. Taxon. 66: 708-717. doi:10.12705/663.11.

Banfi E, Galasso G, Soldano A. 2005. Notes on systematics and taxonomy for the Italian vascular flora 1. Atti Soc Ital Sci Nat Mus Civico Storia Nat Milano. 152: 85-106.

Banfi E, Galasso G, Soldano A. 2011. Notes on systematics and taxonomy for the Italian vascular flora 2. Atti Soc Ital Sci Nat Mus Civico Storia Nat Milano. 146: 219-244.

Banfi E, Soldano A. 1996. Dati tassonomici e nomenclaturali su Poaceae dell'Europa e del Mediterraneo. Atti Soc Ital Sci Nat Mus Civico Storia Nat Milano. 135(2): 379-387.

Bartolucci F, Conti F. 2013. Taxonomic notes on Geum micropetalum Gasp. and Geum heterocarpum Boiss. (Rosaceae) in Italy. Plant Biosyst. 147: 806811. doi:10.1080/11263504.2013.829886.

Bartolucci F, Conti F. 2014. Typification, morphological characterization and distribution of Knautia gussonei (Caprifoliaceae). Phytotaxa. 167: 104110. doi:10.11646/phytotaxa.167.1.6.

Bartolucci F, Conti F. 2016. Alyssum desertorum Stapf (Brassicaceae), new for the Italian flora. Acta Bot Croat. 75: 149-152. doi:10.1515/ botcro-2016-0006.

Bartolucci F, Conti F, lamonico D, Del Guacchio E. 2014a. A new combination in Mcneillia (Caryophyllaceae) for the Italian flora. Phytotaxa. 170: 139140. doi:10.11646/phytotaxa.170.2.8.

Bartolucci F, Domina G. 2014. Typification and taxonomic characterization of Thymus longicaulis C.Presl (Lamiaceae). Ann Bot Fenn. 51: 54-62. doi:10.5735/085.051.0107.

Bartolucci F, Domina G. 2015. The genus Thymus (Lamiaceae) in Sicily. Plant Biosyst. 149: 710-719. doi:10.1080/11263504.2015.1057259.

Bartolucci F, Domina G, Adorni M, Alessandrini A, Angiulli F, Ardenghi NMG, Banfi E, Barberis G, Bedini G, Bonari G, et al. 2016a. Notulae to the Italian native vascular flora: 1. Ital Bot. 1:5-15. doi:10.3897/italianbotanist.1.8780.

Bartolucci F, Domina G, Adorni M, Argenti C, Astuti G, Bangoni S, Buldrini F, Campochiaro MB, Carruggio F, Cecchi L, et al. 2016b. Notulae to the Italian native vascular flora: 2. Ital Bot. 2: 73-92. doi:10.3897/ italianbotanist.2.11060.

Bartolucci F, Domina G, Adorni M, Alessandrini A, Ardenghi NM, Banfi E, Baragliu GA, Bernardo L, Bertolli A, Biondi E, et al. 2017a. Notulae to the Italian native vascular flora: 3. Ital Bot. 3: 29-48. doi:10.3897/ib.3.13200.

Bartolucci F, Domina G, Adorni M, Alessandrini A, Ardenghi NM, Banfi E, Baragliu GA, Bernardo L, Bertolli A, Biondi E, et al. 2017b. Notulae to the Italian native vascular flora: 4. Ital Bot. 4: 43-51. doi:10.3897/ib.4.21693.

Bartolucci F, Galasso G. 2016. A new combination in the genus Liparis (Orchidaceae) for the Italian flora. Phytotaxa. 265: 92. doi:10.11646/ phytotaxa.265.1.10.

Bartolucci F, Galasso G, Rainer H, Wagensommer RP. 2018. Nomenclatural notes and typification of the name Viola pseudogracilis (Violaceae). Phytotaxa.

Bartolucci F, Mráz P. 2016. A new combination in the genus Thymus and clarification on the type material of Th. thracicus (Lamiaceae). Phytotaxa. 263: 161-164. doi:10.11646/phytotaxa.263.2.9.
Bartolucci F, Oriolo G, Casolo V. 2017c. Typification and taxonomy of Thymus carstiensis (Lamiaceae). Plant Biosyst. 151: 737-744. doi:10.1080/112635 04.2017.1320316.

Bartolucci F, Peruzzi L. 2012. Typification of Fritillaria tubiformis Gren. \& Godr. Fritillaria delphinensis f. moggridgei Planch. and Fritillaria delphinensis var. burnatii Planch (Liliaceae) from SW Europe. Candollea. 67: 23-29. doi:10.15553/c2012v671a2.

Bartolucci F, Peruzzi L. 2014. Thymus paronychioides (Lamiaceae), a neglected species from Sicily belonging to section Hyphodromi. Folia Geobot. 49: 83-106. doi:10.1007/s12224-013-9156-2.

Bartolucci F, Peruzzi L, Galasso G, Conti F. 2016c. Checklist aggiornata della flora vascolare autoctona d'Italia [Updated checklist of the Italian native vascular flora]. Notiz Soc Bot Ital: 29-30. Italian.

Bartolucci F, Peruzzi L, Caparelli KF. 2009. A biometric study of Fritillaria montana Hoppe ex W.D.J. Koch s.l. (Liliaceae) shows a single polymorphic species, with no infraspecific taxa. Plant Biosyst. 143: 516-527. doi:10.1080/11263500902722956.

Bartolucci F, Peruzzi L, Passalacqua N. 2013. Taxonomic notes and typification of names within the genus Thymus L. (Lamiaceae). Taxon. 62: 1308-1314. doi:10.12705/626.1.

Bartolucci F, Peruzzi L, Soldano A. 2014b. Notulae: 2064-2069. In: Bartolucci F, Nepi C, Domina G, Peruzzi L, editors. Notulae alla checklist della flora vascolare italiana: 16 (2027-2070) [Notulae to the Italian vascular flora: 16 (2027-2070)]. Inform Bot Ital. 46: 81-83. Italian.

Bartolucci F, Scharfetter A. 2015. Thymus oenipontanus Heinr.Braun ex Borbás (Lamiaceae). In: Raab-Straube E von, Raus T, editors. Euro+Med-Checklist Notulae, 5. Willdenowia. 45: 455.

Bartolucci F, Walter J. 2015. Typification of names in the genus Thymus L. (Lamiaceae). Phytotaxa. 221: 137-147. doi:10.11646/phytotaxa.221.2.3

Bena G. 2001. Molecular phylogeny supports the morphologically based taxonomic transfer of the "medicagoid" Trigonella species to the genus Medicago L. Plant Syst Evol. 229: 217-236. doi:10.1007/s006060170.

Bernardo L, Gargano D, Peruzzi L. 2004. Problemi nella delimitazione delle specie in Campanula L. subsect. Heterophylla (Wit.) Fed. Inform Bot Ital. 36: 516-520.

Bernardo L, Maiorca G, Roma-Marzio F, Peruzzi L. 2018. Alyssum doerfleri Degen (Brassicaceae), new for the Italian flora. Phytotaxa. 334: 10-18. doi:10.11646/phytotaxa.334.1.2.

Bertolli A, Prosser F, editors. 2014. Segnalazioni floristiche tridentine IX. Ann Mus Civici-Rovereto. 29: 131-174.

Blöch C, Dickoré WB, Samuel R, Stuessy T. 2010. Molecular phylogeny of the Edelweiss (Leontopodium, Asteraceae - Gnaphalieae). Edinburgh J Bot. 67: 235-264. doi:10.1017/S0960428610000065.

Borzatti von Loewenstern A, Giordani T, Astuti G, Andreucci A, Peruzzi L. 2013. Phylogenetic relationships of Italian Bellevalia species (Asparagaceae), inferred from morphology, karyology and molecular systematics. Plant Biosyst. 147: 776-787. doi:10.1080/11263504.2013.829884.

Botschantzev VP. 1975. Species subsectionis Vermiculatae Botsch. sectionis Caroxylon (Thunb.) Fenzl generis Salsola L. Novosti Sist Nizsh Rast. 12: 160-194.

Boudrie M, Viane RLL. 2012. Lectotypification, orthographe et auteurs d'Asplenium foreziense Legrand ex Magnier (Aspleniaceae, Pteridophyta). Bull Lias Soc Bot Alsace, Num Spec: 113-128.

Boulos L. 1995. Flora of Egypt. Checklist. Cairo: Al Hadara Publishing.

Bovio M. 2014. Flora vascolare della Valle d'Aosta [Vascular flora of Valle d'Aosta]. Sarre: Testolin editore. Italian.

Brullo S. 1993. Campanula marcenoi (Campanulaceae), a new species from Sicily. Candollea. 48: 493-500.

Brullo C, Brullo S, Gaskin JF, Giusso G, Hrusa GF, Salmeri C. 2015a. A new species of Kali (Salsoloideae, Chenopodiaceae) from Sicily, supported by molecular analysis. Phytotaxa. 201: 256-277. doi:10.11646/phytotaxa.201.4.2.

Brullo C, Brullo S, Giusso del Galdo G, Guarino R, lamonico D. 2013. II genere Salsola s.l. (Chenopodiaceae) in Italia [The genus Salsola s.l. (Chenopodiaceae) in Italy]. In: Peccenini S, Domina G, editors. Contributi alla ricerca floristica in Italia [Contributions to the floristics in Italy]. Palermo: Euroservice Punto Grafica; p. 35-38. Italian.

Brullo S, Guarino R. 2017. Allium L. In: Pignatti S, editor. Flora d'Italia 1 [Flora of Italy 1]. Edagricole: Milano. p. 238-268. Italian. 
Brullo S, Guglielmo A, Pavone P, Salmeri C. 2008. Taxonomic study on Allium dentiferum Webb \& Berthel. (Alliaceae) and its relations with allied species from the Mediterranean. Taxon. 57: 243-253. doi:10.2307/25065965.

Brullo S, Tomaselli V, Wagensommer RP. 2015b. A new species of Odontites (Orobanchaceae) from southern Italy. Phytotaxa. 213: 271-281. doi:10.11646/phytotaxa.213.3.7.

Browicz K. 1963. The genus Colutea L. A monograph. Monogr Bot. 14:61-136. Brundu G, Peruzzi L, Domina G, Bartolucci F, Galasso G, Peccenini S, Raimondo FM, Albano A, Alessandrini A, Banfi E, et al. 2017. At the intersection of cultural and natural heritage: distribution and conservation of the type localities of Italian endemic vascular plants. Biol Conserv. 214: 109-118. doi:10.1016/j.biocon.2017.07.024.

Businský R, Kirschner J. 2006. Nomenclatural notes on the Pinus mugo complex in Central Europe. Phyton (Horn) 46: 129-139.

Businský R, Kirschner J. 2010. Pinus mugo and P. uncinata as parents of hybrids a taxonomic and nomenclatural survey. Phyton (Horn): 27-57.

Buttler KP, Hand R. 2008. Liste der Gefäßpflanzen Deutschlands [List of vascular plants of Germany]. Kochia Beih. 1: 1-107. German.

Calvo J, Álvarez I, Aedo C. 2015. Systematics of Senecio section Crociseris (Compositae, Senecioneae). Phytotaxa. 211: 1-105. doi:10.11646/ phytotaxa.211.1.1.

Campanile G. 1926. Sopra alcune specie di Cuscuta della sezione Clistogrammica [On some species of Cuscuta section Clistogramma]. Ann Bot (Roma) 16: 357-379. Italian.

Carine MA, Francisco-Ortega J, Santos-Guerra A, Russell SJ. 2004 Relationships of island and continental floras: molecular evidence for multiple colonisations into Macaronesia and subsequent backcolonisation of the continent in Convolvulus L. Am J Bot. 91: 1070-1085. doi:10.3732/ajb.91.7.1070.

Carlesi V, Peruzzi L. 2012. The genus Taraxacum (Asteraceae, Cichorieae) in Italy IV. Two new species of T. sect. Palustria. Willdenowia. 42: 191-197. doi:10.3372/wi.42.42204.

Carta A, Savio L, Bedini G, Peruzzi L, Fisogni A, Galloni M. 2016. All in an afternoon: mixed mating system in one-day lasting flowers of Hypericum elodes (Hypericaceae). Plant Biosyst. 150(5): 1001-1009.

Catalán P, Müller J, Hasterok R, Jenkins G, Mur LA, Langdon T, Betekhtin A, Siwinska D, Pimentel M, López-Álvarez D. 2012. Evolution and taxonomic split of the model grass Brachypodium distachyon. Ann Bot (Oxford) 109: 385-405. doi:10.1093/aob/mcr294.

Catalán P, López-Álvarez D, Bellosta C, Villar L. 2016. Updated taxonomic descriptions, iconography, and habitat preferences of Brachypodium distachyon, B. stacei, and B. hybridum (Poaceae). An Jard Bot Madrid. 73: e028. doi:10.3989/ajbm.2428.

Cecchi L, Selvi F. 2017. Flora Critica d'Italia. Boraginaceae - Boragineae, vers. 1.0. doi:10.17773/Fl_Ita_Boragineae1.0. http://www.floraditalia.it.

Celesti-Grapow L, Alessandrini A, Arrigoni PV, Banfi E, Bernardo L, Bovio M, Brundu G, Cagiotti MR, Camarda I, Carli E, et al. 2009. Inventory of the non-native flora of Italy. Plant Biosyst. 143: 386-430. doi:10.1080/11263500902722824.

Christenhusz MJM, Govaerts R, David JC, Hall T, Borland K, Roberts PS, Tuomisto A, Buerki S, Chase MW, Fay MF. 2013. Tiptoe through the tulips - cultural history, molecular phylogenetics and classification of Tulipa (Liliaceae). Bot J Linn Soc. 172: 280-328. doi:10.1111/boj.12061.

Christenhusz MJM, Reveal JL, Farjon A, Gardner MF, Mill RR, Chase MW. 2011. A new classification and linear sequence of extant gymnosperms. Phytotaxa. 19: 55-70. doi:10.11646/phytotaxa.19.1.3.

Conti F. 2010. A new species of Lathyrus L. (Fabaceae) from Central Apennine (Italy). Plant Biosyst. 144: 814-818. doi:10.1080/11263504.2010.523931.

Conti F, Abbate G, Alessandrini A, Blasi C, editors. 2005. An annotated checklist of the Italian vascular flora. Rome: Palombi Editori.

Conti F, Alessandrini A, Bacchetta G, Banfi E, Barberis G, Bartolucci F, Bernardo L, Bouvet D, Bovio M, Del Guacchio E, et al. 2007. Integrazioni alla checklist della flora vascolare italiana [Integrations to the Italian vascular flora]. Nat Vicentina. 10(2006): 5-74. Italian.

Conti F, Bartolucci F. 2012. Two new combinations in Malva (Malvaceae). Ann Bot Fenn. 49: 123-124. doi:10.5735/085.049.0121.

Conti F, Bartolucci F. 2017. Ranunculus giordanoi sp. nov. from the R. auricomus complex (Ranunculaceae), central Apennines (Italy). Nord J Bot. 35: 322 327. doi:10.1111/njb.01433.
Conti F, Bartolucci F, Tomović G, Lakušić D. 2012. Jacobaea vulgaris subsp. gotlandica (Compositae), new for Italy and Montenegro. Bot Serbica. 36: 145-147.

Conti F, Bernardo L, Velari TC, Kosovel V, Feoli CL. 2014. Morphometric and karyological study of Genista sericea (Cytiseae-Fabaceae). Phytotaxa. 181: 61-78. doi:10.11646/phytotaxa.181.2.1.

Conti F, Di Santo D, Giovi E, Tinti D. 2008. Goniolimon italicum Tammaro, Pignatti \& Frizzi. Inform Bot Ital. 40(Suppl. 1): 79-81.

Conti F, Falcinelli F, Palermo D, Paolucci M, Paris P, Domina G, Manzi A, Gallo L, Tondi G, Pennesi R, et al. 2018a. Integrazioni alla flora vascolare dell'Italia centrale. Secondo contributo. Nat Hist Sci.

Conti F, Giordano C, Moraldo B, Ricceri C. 2011. Contribution to the taxonomy of the Italian and Balkanic taxa in the Centaurea rupestris group (Asteraceae). Ann Bot Fenn. 48: 193-218.

Conti F, Guglielmone L, Pennesi R, Bartolucci F. 2016. Typification of the names Astragalus vulnerarioides, basionym of Anthyllis vulneraria subsp. vulnerarioides (Fabaceae) and notes on its distribution. Phytotaxa. 261: 298-300. doi:10.11646/phytotaxa.261.3.12.

Conti F, Pennesi R, Uzunov D, Brachetti L, Bartolucci F. 2018b. A new species of Oxytropis (Fabaceae) from Central Apennine (Italy). Phytotaxa.

Conti F, Uzunov D. 2011. Crepis magellensis F.Conti \& Uzunov (Asteraceae), a new species from Central Apennine (Abruzzo, Italy). Candollea. 66: 81-86. doi:10.15553/c2011v661a5.

Crespo MB, Martínez-Azorín M, Mavrodiev EV. 2015. Can a rainbow consist of a single colour? A new comprehensive generic arrangement of the Iris sensu latissimo clade (Iridaceae), congruent with morphology and molecular data. Phytotaxa. 232: 1-78. doi:10.11646/phytotaxa.232.1.1.

Cristofolini G, Troì A. 2006. A reassessment of the sections of the genus Cytisus Desf. (Cytiseae, Leguminosae). Taxon. 55: 733-746. doi:10.2307/25065647.

D'Antraccoli M, Roma-Marzio F, Astuti G, Maccioni S, Peruzzi L. 2017. Lectotypification of the name Fimbrystilis cioniana (Cyperaceae). Phytotaxa. 303: 194-196. doi:10.11646/phytotaxa.303.2.10.

Danihelka J, Chrtek J Jr, Kaplan Z. 2012. Checklist of vascular plants of the Czech Republic. Preslia. 84: 647-811.

Das S, lamonico D. 2014. Amaranthus bengalense (Amaranthaceae) a new species from India, with taxonomical notes on A. blitum aggregate. Phytotaxa. 181: 293-300. doi:10.11646/phytotaxa.181.5.4.

Del Guacchio E, Caputo P. 2013a. Taxonomic notes on Asperula sect. Cynanchicae (Rubiaceae). Taxon. 62: 394-400. doi:10.12705/622.9.

Del Guacchio E, Caputo P. 2013b. (2136-2137) Proposal to reject the names Asperula aristata subsp. scabra and A. longiflora (A. aristata subsp. longiflora) (Rubiaceae). Taxon. 62: 409-410. doi:10.12705/622.24.

Del Guacchio E, Gargiulo R, Caputo P. 2017. Asperula calabra (Rubiaceae) and allied taxa in southern Apennines, Italy. Plant Biosyst. 151: 352-360. doi:1 0.1080/11263504.2016.1174175

De Castro O, Innangi M, Di Maio A, Menale B, Bacchetta G, Pires M, Noble V, Gestri G, Conti F, Peruzzi L. 2016. Disentangling species relationships in a hotspot of diversity: the butterworts (Pinguicula L., Lentibulariaceae) endemic to Italy. PLoS ONE. 11(12): e0167610. doi:10.1371/journal. pone. 0167610.

Desfayes M. 2011. Notulae alla checklist della flora vascolare italiana 11: (1778-1780) [Notulae to the Italian native vascular flora: 11 (1778-1780)]. Inform Bot Ital. 43: 131-132. Italian.

Devos N, Raspé O, Jacquemart A-N, Tyteca D. 2006. On the monophyly of Dactylorhiza Necker ex Nevski (Orchidaceae): is Coeloglossum viride (L.) Hartman a Dactylorhiza? Bot J Linn Soc. 152: 261-269. doi:10.1111/j.10958339.2006.00561.x.

Dillenberger MS, Kadereit JW. 2014. Maximum polyphyly: multiple origins and delimitation with plesiomorphic characters require a new circumscription of Minuartia (Caryophyllaceae). Taxon. 63: 64-88. doi:10.12705/631.5.

Dillenberger MS, Kadereit JW. 2015. A revision of Facchinia (Minuartia s.l., Caryophyllaceae). Edinburgh J Bot. 72: 353-389. doi:10.1017/ S0960428615000153.

Dimopoulos P, Raus Th, Bergmeier E, Constantinidis Th, latrou G, Kokkini S, Strid A, Tzanoudakis D. 2013. Vascular plants of Greece: an annotated checklist. Berlin; Athens: Botanic Garden and Botanical Museum BerlinDahlem; Hellenic Botanical Society. 
Dimopoulos P, Raus Th, Bergmeier E, Constantinidis Th, latrou G, Kokkini S, Strid A, Tzanoudakis D. 2016. Vascular plants of Greece: an annotated checklist. Supplement. Willdenowia. 46: 301-347. doi:10.3372/ wi.46.46303.

Di Pietro R, Di Marzio P, Misano G, Silletti G, Wagensommer RP, Fortini P. 2016. Evidence from multivariate morphometric study of the Quercus pubescens complex in southeast Italy. Bot Serbica. 40: 83-10. doi:10.5281/ zenodo.48865.

Di Pietro R, Kuzmanović N, lamonico D, Lakušić D. 2017. Nomenclatural and taxonomic notes on Sesleria sect. Argenteae (Poaceae). Phytotaxa. 309: 101-117. doi:10.11646/phytotaxa.309.2.1.

Di Pietro R, Viscosi V, Peruzzi L, Fortini P. 2012. A review of the application of the name Quercus dalechampii. Taxon. 61: 1311-1316.

Doll R. 1973. Revision der sect. Erythrosperma Dahlst. emend. Lindb.f. der Gattung Taraxacum Zinn [Revision of the sect. Erythrosperma Dahlst emend. Lindb.f. of the genus Taraxacum]. Feddes Repert 83. p. 673-740. German.

Domina G. 2017. Phelipanche. In: Raab-Straube von E, Raus T, editors. Euro+Med-Checklist Notulae, 7. Willdenowia. 47: 89-96. doi: 10.3372/ wi.47.47112

Domina G, Bazan G, Campisi P, Greuter W. 2015. Taxonomy and conservation in higher plants and bryophytes in the Mediterranean area. Biodivers. J 6: 197-204.

Domina G, Giusso Del Galdo G, Gargano D, Labra M, Peccenini S, Peruzzi L, Raimondo FM. 2012. The Italian Loci Classici Census. Taxon. 61: 13511353.

Domina G, Greuter W, Mazzola P, Raimondo FM. 2014. Names of Italian vascular plants published by Michele Lojacono Pojero. Flora Medit. 24: 215-232.

Domina G, Greuter W, Raimondo FM. 2016. Types of names of taxa belonging to the Centaurea cineraria group (Compositae) described from Sicily. Willdenowia. 46: 23-26. doi:10.3372/wi.46.46102.

Domina G, Greuter W, Raimondo FM. 2017a. A taxonomic reassessment of the Centaurea busambarensis complex (Compositae, Cardueae), with description of a new species from the Egadi Islands (W Sicily). Israel J Plant Sci. doi:10.1080/07929978.2016.1257146.

Domina G, Scafidi F, Gargano ML. 2017b. Typification of the name Erodium soluntinum Tod. (Geraniaceae) and its taxonomic implications. Phytotaxa. 329: 219-292. doi:10.11646/phytotaxa.329.3.12.

Domina G, Soldano A. 2015. Orobanche apuana (Orobanchaceae) a new species endemic to Italy. Phytotaxa. 207: 163-171. doi:10.11646/ phytotaxa.207.2.2.

Dunkel FG. 2010. The Ranunculus auricomus L. complex (Ranunculaceae) in Northern Italy. Webbia. 65: 179-227. doi:10.1080/00837792.2010.10670 873.

Dunkel FG. 2011. The Ranunculus auricomus L. complex (Ranunculaceae) in Central and Southern Italy, with additions to North Italian taxa. Webbia. 66: 165-193. doi:10.1080/00837792.2011.10670895.

Dunkel FG, Poldini L. 2016. Ranunculus farraensis sp. nov., a new species of the Ranunculus auricomus complex (Ranunculaceae) in northeastern Italy - revealed by herbarium revision and still extant. Webbia. 71: 197-201. do i:10.1080/00837792.2016.1189129.

Euro+Med. 2006 onwards. Euro+Med Plantbase - the information resource for Euro-Mediterranean plant diversity. http://ww2.bgbm.org/ EuroPlusMed/.

Fenaroli F, Pistarino A, Peruzzi L, Cellinese N. 2013. Campanula martinii (Campanulaceae), a new species from northern Italy. Phytotaxa. 111: 27-38. doi:10.11646/phytotaxa.111.1.2.

Fenu G, Bacchetta G, Giacanelli V, Gargano D, Montagnani C, Orsenigo S, Cogoni D, Rossi G, Conti F, Santangelo A, et al. 2017. Conserving plant diversity in Europe: outcomes, criticisms and perspectives of the Habitats Directive application in Italy. Biodivers Conserv. 26: 309-328. doi:10.1007/ s10531-016-1244-1.

Festi F. 2000. Chiave d'identificazione per le specie italiane del genere Alchemilla L. (Rosaceae). Ann Mus Civici-Rovereto. 14(1): 105-174.

Frajman B, Schönswetter P. 2017. Amphi-Adriatic distributions in plants revisited: Pleistocene trans-Adriatic dispersal in the Euphorbia barrelieri group (Euphorbiaceae). Bot J Linn Soc. 185(2): 240-252. doi:10.1093/ botlinnean/box055.
Fraser-Jenkins CR. 2007. The species and subspecies in the Dryopteris affinis group. Fern Gaz. 18: 1-26.

Fröhner SE, Bona E, Federici G, Martini F. 2012. Contributo alla conoscenza del gen. Alchemilla sulle Alpi italiane: Alpi Retiche Meridionali, Prealpi Lombarde Centrali e Orientali. Inform Bot Ital. 44: 3-73.

Fridlender A. 2009. Colchicum verlaqueae Fridlender: un colchique nouveau endémique du littoral sarde [Colchicum verlaqueae Fridlender: a new endemic Colchicum from Sardinia]. Bull Mens Soc Linn Lyon. 78: 111-117. French.

Fridlender A. 2016. Corrigenda to "Is it possible to identify Colchicum neapolitanum s.l. and C. autumnale s.l. in vegetative stage? Biometry and flow cytometry approaches". Bot Serbica. 38: 43-56.

Galasso G, Bartolucci F. 2014. Notula: 2070. In: Barberis G, Nepi C, Peccenini S, Peruzzi L, editors. Notulae alla checklist della flora vascolare italiana [Notulae to the Italian native vascular flora]: 17 (2027-2070). Inform Bot Ital. 46: 83. Italian.

Galasso G, Bartolucci F. 2015. Four new combinations in Jacobaea Mill. (Asteraceae, Senecioneae) for the European flora. Nat Hist Sci. 2: 95-96. doi:10.4081/nhs.2015.246.

Galasso G, Bartolucci F, Peruzzi L, Ardenghi NMG, Banfi E, Celesti-Grapow L, Conti F. 2016a. Checklist aggiornata della flora vascolare alloctona d'Italia [Updated checklist of the Italian alien vascular flora]. Notiz Soc Bot Ital: 49-50. Italian.

Galasso G, Domina G, Ardenghi NMG, Arrigoni P, Banfi E, Bartolucci F, Bonari G, Buccomino G, Ciaschetti G, Conti F, et al. 2016b. Notulae to the Italian alien vascular flora: 2. Ital Bot. 2: 55-71. doi:10.3897/italianbotanist.2.11144.

Galasso G, Montoleone E, Federico C. 2014. Persicaria senegalensis (Polygonaceae), entità nuova per la flora italiana, e chiave di identificazione delle specie del genere Persicaria in Italia. Nat Hist Sci. 1: 13-18. doi:10.4081/nhs.2014.62.

Gallo L, Conti F. 2015. On the true identity of the plants recently referred to Sedum nevadense (Crassulaceae) in Abruzzo (Italy). Phytotaxa. 239: 43-54. doi:10.11646/phytotaxa.239.1.4.

Gargiulo R, Del Guacchio E, Caputo P. 2015. Phylogenetic reconstruction of Asperula sect. Cynanchicae (Rubiaceae) reveals a mosaic of evolutionary histories. Taxon. 64: 754-769. doi:10.12705/644.7.

Garrido JL, Fenu G, Mattana E, Bacchetta G. 2012. Spatial genetic structure of Aquilegia taxa endemic to the island of Sardinia. Ann Bot (Oxford). 109: 953-964. doi:10.1093/aob/mcs011.

Gatto A, De Paola D, Bagnoli F, Vendramin GG, Sonnante G. 2013. Population structure of Cynara cardunculus complex and the origin of the conspecific crops artichoke and cardoon. Ann Bot (Oxford). 112: 855-865. doi:10.1093/aob/mct150.

Giardina G, Raimondo FM, Spadaro V. 2007. A catalogue of plants growing in Sicily. Bocconea. 20: 5-582.

GIROS. 2016. Orchidee d'Italia. Guida alle orchidee spontanee [Orchids of Italy. Guide to Italian native orchids]. Milano: Ed II Castello. Italian.

Glon HE, Shiels DR, Linton E, Starr JR, Shorkey AL, Fleming S, Lichtenwald SK, Schick ER, Pozo D, Monfils AK. 2017. A five gene phylogenetic study of Fuireneae (Cyperaceae) with a revision of Isolepis humillima. Syst Bot. 42: 26-36. doi:10.1600/036364417X694601.

Griebl N. 2010. Die Orchideen der Abruzzen [The orchids from Abruzzo]. Ber Arbeitskreis Heimische Orchid. 27: 112-159. German.

Güner A, Aslan S, Ekim T, Vural M, Babaç MT, editors. 2012. Türkiye Bitkileri Listesi (Damarlı Bitkiler) [List of Turkey plants]. Istanbul: Nezahat Gökyiğit Botanic Garden and Flora Research Society Publishing. Turkish.

Güner A, Özhatay N, Ekim T, Başer KHC. 2000. Flora of Turkey and the East Aegean Islands. Vol. 11. Edinburgh: Edinburgh University Press.

Hamilton CW, Reichard SH. 1992. Current practice in the use of subspecies, variety, and forma in the classification of wild plants. Taxon. 41: 485498.

Hand R. 2001. Revision der in Europa vorkommenden Arten von Thalictrum subsectio Thalictrum (Ranunculaceae). Bot Naturschutz Hessen. 9: 1-358.

Harber J, Raab-Straube E von. 2015. Berberidaceae. In: Euro+Med Plantbase - the information resource for Euro-Mediterranean plant diversity. http:// ww2.bgbm.org/EuroPlusMed/.

Harpke D, Carta A, Tomović G, Ranđelović V, Ranđelović N, Blattner FR, Peruzzi L. 2015. Phylogeny, karyotype evolution and taxonomy of Crocus ser. Verni (Iridaceae). Plant Syst Evol. 301: 309-325. doi:10.1007/s00606014-1074-0. 
Harpke D, Kerndorff H, Pasche E, Peruzzi L. 2016. Neotypification of the name Crocus biflorus Mill. (Iridaceae) and its consequences in the taxonomy of the genus. Phytotaxa. 260: 131-143. doi:10.11646/phytotaxa.260.2.3.

Harpke D, Peruzzi L, Kerndorff H, Karamplianis T, Constantinidis T, Ranđelović V, Ranđelović N, Jušković M, Pasche E, Blattner FR. 2014. Phylogeny, geographic distribution and new taxonomic circumscription of the Crocus reticulatus species group (Iridaceae). Turk J Bot. 38: 1182-1198. doi:10.3906/bot-1405-60.

Hassemer G, Meudt HM, Rønsted N. 2017. Nomenclatural and taxonomic notes on Mediterranean narrow-leaved plantains (Plantago section Maritima, Plantaginaceae). Webbia. 72: 197-205. doi:10.1080/00837792 .2017 .1349066 .

Hempel W. 2011. Revision und Phylogenie der Arten der Gattung Melica L. (Poaceae) in Eurasien und Nordafrika [Revision and phylogeny of the genus Melica L. (Poaceae) in Eurasia and North Africa]. Feddes Repert. 122: 1-253. German.

Hernández-Ledesma P, Berendsohn WG, Borsch T, von Mering S, Akhani H, Arias S, Castañeda-Noa I, Eggli U, Eriksson R, Flores-Olvera H, et al. 2015. A taxonomic backbone for the global synthesis of species diversity in the angiosperm order Caryophyllales. Willdenowia. 45: 281-383. doi:10.3372/ wi.45.45301.

Hauenschild F, Favre A, Salazar GA, Muellner-Riehl AN. 2016. Analysis of the cosmopolitan buckthorn genera Frangula and Rhamnus s.l. supports the description of a new genus, Ventia. Taxon. 65: 65-78. doi:10.12705/651.5.

Herrando-Moraira S, Carnicero P, Blanco-Moreno JM, Sáez L, Véla E, Vilatersana R, Galbany-Casals M. 2017. Systematics and phylogeography of the Mediterranean Helichrysum pendulum complex (Compositae) inferred from nuclear and chloroplast DNA and morphometric analyses. Taxon. 66: 909-933. doi:10.12705/664.7.

lamonico D. 2010a. Malva subovata subsp. bicolor, comb. \& stat. nov. (Malvaceae). Ann Bot Fenn. 47: 312-314. doi:10.5735/085.047.0409.

lamonico D. 2010b. Confirmation of the occurrence of Chenopodium strictum subsp. strictum (Amaranthaceae s. I.) in Italy. Phyton (Horn) 49: 235-240.

lamonico D. 2012. Note sul genere Polycnemum (Amaranthaceae) in Italia. In: Peccenini S, Domina G, Salmeri C, editors. Flora vascolare d'Italia: studi biosistematici, taxa endemici e loci classici [Notes on the genus Polycnemum in Italy]. Palermo: Euroservice Punto Grafica. p. 31-32. Italian.

lamonico D. 2013a. Taxonomical and chorological study on the Central Mediterranean Basin endemic Arenaria bertolonii Fiori \& Paol. (Caryophyllaceae).. Plant Biosyst. 147: 923-930. doi:10.1080/11263504.2 012.753956.

lamonico D. 2013b. Chenopodium L. sensu lato (Chenopodiaceae, Chenopodioideae) nella flora vascolare italiana: chiave analitica dei generi. Inform Bot Ital. 45: 83-88.

lamonico D. 2013c. Studies on the genus Atriplex L. (Amaranthaceae) in Italy. V. Atriplex tornabenei. Phytotaxa. 145: 54-60. doi:10.11646/ phytotaxa.145.1.6.

lamonico D. 2013d. Polycnemum verrucosum (Amaranthaceae), first record for the Italian flora and comparison with related species $P$. arvense. Hacquetia. 12: 5-9. doi:10.2478/HACQ-2013-0001.

lamonico D. 2014a. Lavatera stenopetala subsp. plazzae comb. et stat. nov., endemic taxon from north-western Sardinia (Italy). Plant Ecol Evol. 147: 293-298. doi:10.5091/plecevo.2014.920.

lamonico D. 2014b. Arenaria tenuifolia versus Arenaria hybrida (Caryophyllaceae): nomenclatural study and taxonomic implications. Phytotaxa. 173: 235-240. doi:10.11646/phytotaxa.173.3.6.

lamonico D. 2014. Taxonomical, morphological, ecological and chorological notes on Oxybasis chenopodioides and O. rubra (Chenopodiaceae) in Italy. Hacquetia. 13: 297-302. doi:10.2478/hacq-2014-0004.

lamonico D. 2015. Taxonomic revision of the genus Amaranthus (Amaranthaceae) in Italy. Phytotaxa. 199: 1-84. doi:10.11646/phytotaxa. 199.1.1.

lamonico D. 2016a. Nomenclatural notes and morphological observations on Malva subovata s.l. (Malvaceae). Phytotaxa. 275: 23-34. doi:10.11646/ phytotaxa.275.1.3.

lamonico D. 2016b. Nomenclatural notes on four Linnaean names in Arenaria (Caryophyllaceae). Taxon. 65: 610-616. doi:10.12705/653.13.

lamonico D. 2016c. A new name in Sagina, Sagina alexandrae (Caryophyllaceae). Phytotaxa. 282: 164-165. doi:10.11646/phytotaxa. 282.2.8. lamonico D. 2017. Agrimonia eupatoria subsp. major stat. nov. (Rosaceae) and notes on the Agrimonia eupatoria aggregate. Phytotaxa. 298: 165-172. doi:10.11646/phytotaxa.298.2.6.

lamonico D, Bartolucci F, Conti F. 2016a. New combinations in the genus Siler (Apiaceae) for the Italian Flora. Phytotaxa. 268: 89-90. doi:10.11646/ phytotaxa.268.1.9.

lamonico D, Bartolucci F, Peruzzi L. 2015. Malva stenopetala subsp. plazzae (Atzei) lamonico, Bartolucci \& Peruzzi, comb. nov. In: Raab-Straube E von, Raus Th, editors. Euro+Med-Checklist Notulae, 4 [Notulae ad floram euromediterraneam pertinentes 33]. Willdenowia. 45: 123.

lamonico D, Domina G. 2015. Nomenclatural notes on the Polycarpon tetraphyllum aggregate (Caryophyllaceae). Plant Biosyst. 149: 720-727. doi:10.1080/11263504.2015.1057260.

lamonico D, Giovi E, Iberite M, Abbate G. 2011. Typification of Trifolium latinum Sebast. (Fabaceae) and comparison with related species. Ann Bot Fennici. 48: 459-464.

lamonico D, Guarino R, llardi V, Pignatti S. 2016b. A revision of Helichrysum panormitanum s.l. (Asteraceae) in the Italian and Maltese floras. Phytotaxa. 286: 207-210. doi:10.11646/phytotaxa.286.3.10.

lamonico D, Vallariello R, Del Guacchio E. 2017. Nomenclatural and distributional remarks on Limonium tenoreanum (Plumbaginaceae), narrow endemic to southern Italy. Nord J Bot. 35: 445-448. doi:10.1111/ njb.01313.

Iberite $M$, Abbate $G$, lamonico D. 2017. Vicia incisa (Fabaceae): taxonomical and chorological notes. Ann Bot (Roma). 7: 57-65. doi:10.4462/ annbotrm-13842.

Iberite M, lamonico D. 2016. Sull'identità di Salicornia veneta (Amaranthaceae) [On the identity of Salicornia veneta (Amaranthaceae)]. Notiz Soc Bot Ital: 51-52. Italian.

Iberite M, lamonico D, Valletta A. 2015. Revised typification of the name Bupleurum gracile DC. var. rollii Montel. (Apiaceae) and comparison with B. asperuloides Heldr., B. gracile D'Urv., B. marschallianum C. A. Mey and B. uechtritzianum S. Stoyanov. Plant Biosyst. 149: 78-87. doi:10.1080/11263 504.2013.814601.

Inocencio C, Rivera D, Obón MC, Alcaraz F, Barreña J-A. 2006. A systematic revision of Capparis section Capparis (Capparaceae). Ann Missouri Bot Gard. 93: 122-149. doi:10.3417/0026-6493(2006) 93[122:ASROCS]2.0.CO;2.

IPNI. 2012 onwards. The International Plant Names Index. http://www.ipni. org.

Jauzein P, Tison J-M. 2001. Étude analytique du genre Allium L., sous-genre Codonoprasum (Reichenb.) Zahar., section Codonoprasum Reichenb. en France. J Bot Soc Fr. 15: 29-50.

Kadereit G, Piirainen M, Lambinon J, Vanderpoorten A. 2012. Cryptic taxa should have names: reflections in the glasswort genus Salicornia (Amaranthaceae). Taxon. 61: 1227-1239.

Kellogg EA. 2015. The families and genera of vascular plants. Vol. 13. Cham: Springer.

Kato Y, Aioi K, Omori Y, Takahata N, Satta Y. 2003. Phylogenetic analyses of Zostera species based on $r b c \mathrm{~L}$ and matK nucleotide sequences: implications for the origin and diversification of the seagrasses in Japanese waters. Genes Genet Syst. 78: 329-342. doi:10.1266/ggs.78.329.

Kirschner J. 2002. Species Plantarum. Flora of the world. Juncus subg. Agathryon. Vol. 8. Canberra: Australian Biological Resources Study. VIII + $192 \mathrm{p}$.

Kirschner J, Raab-Straube E von. 2017. Valeriana officinalis aggr. In: RaabStraube E von, Henning T, editors. Valerianaceae. Euro+Med Plantbase the information resource for Euro-Mediterranean plant diversity. http:// ww2.bgbm.org/EuroPlusMed/.

Kirschner J, Štepánek J. 1998. A monograph of Taraxacum sect. Palustria. Průhonice: Institute of Botany, Academy of Sciences, Czech Republic.

Kirschner J, Štepánek J, Greuter W. 2007. Taraxacum. In: Greuter W, Raab-Straube E von, editors. Compositae. Euro+Med Plantbase - the information resource for Euro-Mediterranean plant diversity. http://ww2. bgbm.org/EuroPlusMed/.

Klak C, Hanáček P, Bruyns PV. 2017. Disentangling the Aizooideae: new generic concepts and a new subfamily in Aizoaceae. Taxon. 66: 11471170. doi:10.12705/665.9.

Koch MA, Karl R, German DA. 2017. Underexplored biodiversity of Eastern Mediterranean biota: systematics and evolutionary history of the genus Aubrieta (Brassicaceae). Ann Bot. 119: 39-57. 
Kool A, Thulin M. 2017. A giant spurrey on a tiny island: on the phylogenetic position of Sanctambrosia manicata (Caryophyllaceae) and the generic circumscriptions of Spergula, Spergularia and Rhodalsine. Taxon. 66: 615622. doi:10.12705/663.6.

Lauber K, Wagner G. 2007. Flora Helvetica. Bern: Haupt Verlag.

Li Q-Y, Guo W, Liao W-B, Macklin JA, Li J-H. 2012. Generic limits of Pyrinae: insights from nuclear ribosomal DNA sequences. Bot Stud. 53: 151-164.

Lo EYY, Donoghue MJ. 2012. Expanded phylogenetic and dating analysis of the apples and their relatives (Pyrinae, Rosaceae). Mol Phyl Evol. 63: 230-243. doi:10.1016/j.ympev.2011.10.005

Lomolino MV. 2004. Conservation biogeography. In: Lomolino MV, Heaney LR, editors. Frontiers of biogeography: new directions in the geography of nature. Sunderland, MA: Sinauer Associates. p. 293-296.

Lucchese F. 1996. Correzioni ed aggiunte alla flora del Molise. Ann Bot (Roma). 54: 1-294.

Luebert F, Cecchi L, Frohlich MW, Gottschling M, Guilliams CM, HasenstabLehman KE, Hilger HH, Miller JS, Mittelbach M, Nazaire M, et al. 2016. Familial classification of the Boraginales. Taxon. 65: 502-522. doi:10.12705/653.5.

Lye KA. 2003. Schoenoplectiella Lye, gen. nov. (Cyperaceae). Lidia. 6: 20-29.

Masin R, Tietto C. 2006. Flora vascolare della Provincia di Padova (Italia Nordorientale) [Vascular flora of the province of Padova (North-East Italy)]. Natura Vicentina. 9(2005): 7-103. Italian.

Mattana E, Daws MI, Fenu G, Bacchetta G. 2012. Adaptation to habitat in Aquilegia species endemic to Sardinia (Italy): seed dispersal, germination, and persistence in the soil. Plant Biosyst. 146: 374-383.

Mazur M, Minissale P, Sciandrello S, Boratyński A. 2016. Morphological and ecological comparison of populations of Juniperus turbinata Guss. and J. phoenicea L. from the Mediterranean region. Plant Biosyst. 150: 313-322. doi:10.1080/11263504.2014.994579.

McNeill J, Barrie FR, Buck WR, Demoulin V, Greuter W, Hawksworth DL, Herendeen PS, Knapp S, Marhold K, Prado J, et al., editors. 2012. International Code of Nomenclature for algae, fungi and plants (Melbourne Code) adopted by the Eighteen International Botanical Congress Melbourne, Australia, July 2011. Königstein: Koeltz Scientific Books. (Regnum Vegetabile; 154).

Melai M, Marchetti D, Bernardello R, Peruzzi L. 2012. A new diploid species of Leucanthemum (Asteraceae, Anthemideae) from Liguria (northwestern Italy). Phytotaxa. 66: 27-37. doi:10.11646/phytotaxa.66.1.5.

Mercuri AM, Bandini Mazzanti M, Florenzano A. 2013. Olea, Juglans and Castanea: The OJC group as pollen evidence of the development of human-induced environments in the Italian peninsula. Quat Int. 303: 24-42.

Molero J, Montserrat JM. 2005. Nomenclatura de algunas especies del género Malva Linnaeus (Malvaceae). Fontqueria. 55: 285-292.

Molins A, Bacchetta G, Rosato M, Roselló JA, Mayol M. 2011. Molecular phylogeography of Thymus herba-barona (Lamiaceae): insight into the evolutionary history of the flora of the western Mediterranean islands. Taxon. 60: 1295-1305.

Moore AJ, Dillenberger MS. 2017. A conspectus of the genus Cherleria (Minuartia s.l., Caryophyllaceae). Willdenowia. 47: 5-14. doi:10.3372/ wi.47.47101.

Morretti F, Puppi G, Giuliani C, Conti F. 2015. Heterostyly in Goniolimon italicum (Plumbaginaceae), endemic to Abruzzo (central Apennines, Italy). Anales Jard Bot Madrid. 72: 1-5. doi:10.3989/ajbm.2365.

Mosyakin SL. 2017a. The first record of Salsola paulsenii (Chenopodiaceae) in Ukraine, with taxonomic and nomenclatural comments on related taxa. Ukr Bot J. 74: 409-420. doi:10.15407/ukrbotj74.05.409.

Mosyakin SL. 2017b. Notes on taxonomy and nomenclature of Chenopodium acerifolium and C. betaceum (C. strictum auct.) (Chenopodiaceae). Phytotaxa. 324: 139-154. doi:10.11646/phytotaxa.324.2.3.

Mosyakin SL. 2017c. Taxonomic and nomenclatural notes on PonticMediterranean coastal and some Australasian taxa of Salsola (Chenopodiaceae). Ukr Bot J 74: 521-531. doi:10.15407/ukrbotj74.06.521.

Mosyakin SL, Freitag H, Rilke S. 2017. Kali versus Salsola: the instructive story of a questionable nomenclatural resurrection. Israel J Plant Sci. 64: 18-30. doi:10.1080/07929978.2016.1256135.

Mucciarelli M, Rosso P, Noble V, Bartolucci F, Peruzzi L. 2016. A morphometric study and taxonomic revision of Fritillaria tubaeformis species complex
(Liliaceae). Plant Syst Evol. 302: 1329-1343. doi:10.1007/s00606-0161334-2.

Nardi E. 2015. II genere Aquilegia L (Ranunculaceae) in Italia [The genus Aquilegia (Ranunculaceae) in Italy]. Firenze: Edizioni Polistampa. Italian.

Nie Z-L, Funk VA, Meng Y, Deng T, Sun H, Wen J. 2016. Recent assembly of the global herbaceous flora: evidence from the paper daisies (Asteraceae: Gnaphalieae). New Phytol. 209: 1795-1806. doi:10.1111/nph.13740.

Nordenstam B, Greuter W. 2006. Jacobaea Mill. Willdenowia. 36: 711-713.

Orsenigo S, Astuti G, Bartolucci F, Citterio S, Conti F, Garrido-Becerra JA, Gentili R, Giusso del Galdo G, Jiménez-Martinez JF, Karrer G, et al. 2017. Global and regional IUCN red list assessments: 3. Ital Bot. 3: 83-98. doi:10.3897/italianbotanist.3.13303.

Özhatay N, Koçyiğit M, Yüzbaşıoğlu S, Gürdal B. 2013. Mediterranean flora and its conservation in Turkey, with special reference to Monocot geophytes. Flora Medit. 23: 195-208.

Özhatay N, Kültür Ş, Gürdal B. 2017. Check-list of additional taxa to the supplement flora of Turkey VIII. J Pharm Istanbul. 47: 31-46.

Padilla-García N, Rojas-Andrés BM, López-González N, Castro M, Castro S, Loureiro J, Albach DC, Machon N, Martínez-Ortega MM. 2018. The challenge of species delimitation in the diploid-polyploid complex Veronica subsection Pentasepalae. Mol Phyl Evol. 119: 196-209. doi:10.1016/j.ympev.2017.11.007.

Palermo AM, De Vita A, Peruzzi L, Gargano D, Bernardo L, Musacchio A 2010. Does Plantago brutia Ten. (Plantaginaceae) merit specific rank? Insights from cpDNA and nrDNA data. Plant Biosyst. 144: 573-581. doi:10.1080/11263501003672496.

Passalacqua N, Banfi E, Galasso G. 2014. Banca dati delle piante endemiche italiane: protologhi e loci classici [Database of Italian endemic plants: protologues and loci classici]. In: Alessandrello A, editor, Al Museo per scoprire il mondo. La ricerca scientifica e le esposizioni. Museo Civico di Storia Naturale di Milano [Discovring the World in the mueseum. The scientific research and the exhibitions. Milan Natural Science Museum] 2010-2013. Natura. 104: 33-38. Italian.

Passalacqua NG, Peruzzi L, Pellegrino G. 2008. A biosystematic study of the Jacobaea maritima group (Asteraceae, Senecioneae) in the Central Mediterranean area. Taxon. 57: 893-906

Paule J, Sharbel JF, Dobeš C. 2011. Apomictic and sexual lineages of the Potentilla argentea L. group (Rosaceae): Cytotype and molecular genetic differentiation. Taxon. 60: 721-732

Peccenini S. 2012. The genus Erysimum (Brassicaceae) in Italy, part I. Ann Naturhist Mus Wien B. 112: 95-128.

Peccenini S, Polatschek A. 2014. The genus Erysimum (Brassicaceae) in Italy, part II: description of new species and subspecies. Ann Naturhist Mus Wien B. 116: 107-117.

Peccenini S, Polatschek A. 2016. The genus Erysimum (Brassicaceae) in Italy, part III: key to the species and description of the new species E. apenninum, E. etruscum, E. pignattii. Ann Naturhist Mus Wien B. 118: 147-166.

Perazza G, Decarli M, Filippin P, Bruna A, Regattin L. 2012. Liparis loeselii subsp. nemoralis, a new orchid from Northern-Italy. J Eur Orch. 44: 483-508.

Perazza G, Lorenz R. 2013. Le orchidee dell'Italia nordorientale Atlante corologico e guida al riconoscimento [The orchids of Northeastern Italy. Chorological Atlas and guide to recognition]. Trento: Edizioni Osiride. Italian.

Perrino EV, Wagensommer RP, Medagli P. 2014. Aegilops (Poaceae) in Italy: taxonomy, geographical distribution, ecology, vulnerability and conservation. Syst Biodivers. 12: 331-349. doi:10.1080/14772000.2014.9 09543.

Persson K. 2007. Nomenclatural synopsis of the genus Colchicum (Colchicaceae), with some new species and combinations. Bot Jahrb Syst. 127: 165-242. doi:10.1127/0006-8152/2007/0127-0165.

Persson K. 2008. A new species of Colchicum (Colchicaceae) from southern Italy. Bot Jahrb Syst. 127: 283-288. doi:10.1127/0006-8152/2008/0127 0283.

Peruzzi L. 2007. Notula: 1310. In: Conti F, Nepi C, Scoppola A, editors, Notulae alla checklist della flora vascolare italiana 3: (1267-1310) [Notulae to the checklist of Italian vascular flora: 3 (1267-1310)]. Inform Bot Ital. 39: 252 253. Italian.

Peruzzi L. 2008. Notulae: 1472-1473. In: Nepi C, Peruzzi L, Scoppola A, editors, Notulae alla Checklist della flora italiana 5: (1420-1474) [Notulae 
to the checklist of Italian vascular flora 5: (1420-1474)]. Inform Bot Ital. 40. p. 114-115. Italian.

Peruzzi L. 2011. A new species of Linum perenne group (Linaceae) from Calabria (S Italy). Plant Biosyst. 145: 938-944. doi:10.1080/11263504.20 11.589163

Peruzzi L. 2012. Nomenclatural novelties at sectional level in Gagea (Liliaceae). Atti Soc Tosc Sci Nat Mem Ser B. 118(2011): 23-24.

Peruzzi L. 2014. Miscellaneous typifications of selected species occurring in Calabria (S Italy). Phytotaxa. 178: 50-52. doi:10.11646/phytotaxa.178.1.6.

Peruzzi L. 2016. Crocus heuffelianus (Iridaceae), a new record for the Italian flora. Phytotaxa. 261: 291-294. doi:10.11646/phytotaxa.261.3.10.

Peruzzi L, Adorni M, Dura T, Ghillani L, Pasquali G, Rignanese L, Ronconi D, Teruzzi M. 2012a. Allium cyrilli Ten. (Amaryllidaceae): typification, taxonomy and update of the Italian distribution. Phytotaxa. 71: 53-58. doi:10.11646/phytotaxa.71.1.11.

Peruzzi L, Aquaro G, Caparelli KF, Raimondo FM. 2009. The genus Taraxacum (Asteraceae) in Italy. III. A new species of T. sect. Erythrocarpa (Asteraceae) from Sicily. Flora Medit. 19: 73-79.

Peruzzi L, Astuti G, Carta A, Roma-Marzio F, Dolci D, Caldararo F, Bartolucci F, Bernardo L. 2015d. Nomenclature, morphometry, karyology and SEM cypselae analysis of Carduus brutius (Asteraceae) and its relatives. Phytotaxa. 202: 237-249. doi:10.11646/phytotaxa.202.4.1.

Peruzzi L, Bartolucci F. 2009. Typification of the names in Fritillaria montana complex. Candollea. 64: 133-142.

Peruzzi L, Bartolucci F. 2016. Essere o non essere (una sottospecie): questo è il problema [To be or not to be (a subspecies): this is the question]. Notiz Soc Bot Ital: 61-62. Italian.

Peruzzi L, Bartolucci F, Astuti G, Bernardo L, Conti F. 2013a. Knautia dinarica (Caprifoliaceae): taxonomy, typification and update of the Italian distribution. Phytotaxa. 116: 36-40. doi:10.11646/phytotaxa.116.1.3.

Peruzzi L, Bedini G, Andreucci A. 2012b. Homoploid hybrid speciation in Doronicum L. (Asteraceae)? Morphological, karyological and molecular evidences. Plant Biosyst. 146: 867-877. doi:10.1080/11263504.2011.634 445.

Peruzzi L, Carta A. 2011. Crocus ilvensis sp. nov. (sect. Crocus, Iridaceae), endemic to Elba Island (Tuscan Archipelago, Italy). Nord J Bot. 29: 6-13. doi:10.1111/j.1756-1051.2010.01023.x.

Peruzzi L, Carta A, Garbari F. 2013b. Lectotypification of the name Crocus sativus var. vernus L. (Iridaceae) and its consequences within the ser. Verni. Taxon. 62: 1037-1040. doi:10.12705/625.7.

Peruzzi L, Conti F, Bartolucci F. 2014a. An inventory of vascular plants endemic to Italy. Phytotaxa. 168: 1-75. doi:10.11646/phytotaxa.168.1.1.

Peruzzi L, Conti F, Bartolucci F. 2014b. Notulae: 2098-2099. In: Barberis G, Nepi C, Peccenini S, Peruzzi L, editors, Notulae alla checklist della flora vascolare italiana 18 (2071-2099). Inform Bot Ital. 46: 276-277.

Peruzzi L, Conti F, Bartolucci F. 2015a. A historical perspective on vascular plants endemic to Italy. Atti Soc Tosc Sci Nat Mem Ser B. 121(2014): 89-92.

Peruzzi L, Di Benedetto C, Aquaro G, Caparelli KF. 2008a. The genus Sternbergia Waldst. \& Kit. (Amaryllidaceae) in Italy. Contribution to the cytotaxonomical and morpho-anatomical knowledge. Caryologia. 61: 107-113.

Peruzzi L, Domina G, Bartolucci F, Galasso G, Peccenini S, Raimondo FM, Albano A, Alessandrini A, Banfi E, Barberis G, et al. 2015b. An inventory of the names of vascular plants endemic to Italy, their loci classici and types. Phytotaxa. 196: 1-217. doi:10.11646/phytotaxa.196.1.1.

Peruzzi L, Gestri G. 2013. A new butterwort species (Pinguicula, Lentibulariaceae) from Northern Apennine (Italy). Plant Biosyst. 147: 692-703. doi:10.1080/11263504.2012.756073.

Peruzzi L, Gestri G, Pierini B. 2014c. Morphological variation in Astragalus muelleri (Fabaceae) from Balkan and Italian populations. Flora Medit. 24: 139-144.

Peruzzi L, Innangi M, Tatino F, Santangelo A. 2017. Fritillaria messanensis subsp. gracilis (Liliaceae), a new record for the Italian flora (S Italy). Phytotaxa. 307: 167-170. doi:10.11646/phytotaxa.307.2.11.

Peruzzi L, Jarvis CE, Carta A. 2014b. On the application of the Linnaean names Cucubalus reflexus, Silene nocturna and Silene mutabilis (Caryophyllaceae). Taxon. 63: 651-652.

Peruzzi L, Passalacqua NG, Jarvis CE. 2006. Typification of the accepted names in the Jacobaea maritima Group (Asteraceae). Taxon. 55: 1001-1004.
Peruzzi L, Passalacqua NG. 2008. Taxonomy of the Onosma echioides (L.) L. complex (Boraginaceae) based on morphometric analysis. Bot J Linn Soc. 157: 763-774. doi:10.1111/j.1095-8339.2008.00827.x.

Peruzzi L, Peterson A, Tison J-M, Peterson J. 2008b. Phylogenetic relationships of Gagea Salisb. (Liliaceae) in Italy, inferred from molecular and morphological data matrices. Plant Syst Evol. 276: 219-234.

Peruzzi L, Pierini B, Magrini S, Andreucci A, Marchetti D, Viane R. 2015c. Three new hybrids of Ophioglossum (Ophioglossaceae) from Monte Pisano (Tuscany, Central Italy). Plant Biosyst. 149: 737-746. doi:10.1080/112635 04.2015.1057264.

Peruzzi L, Santangelo A, Jarvis CE. 2013c. Lectotypification of Linnaean names in the Italian endemic flora. Taxon. 62: 827-829.

Peruzzi L, Tison J-M, Peterson A, Peterson J. 2008c. On the phylogenetic position and taxonomic value of Gagea trinervia (Viv.) Greuter and the whole G. sect. Anthericoides A.Terracc. (Liliaceae). Taxon. 57: 1201-1214.

Peterson A, Harpke D, Peruzzi L, Tison J-M, John H, Peterson J. 2010. Gagea bohemica (Liliaceae), a highly variable monotypic species within sect. Didymobulbos. Plant Biosyst. 144: 308-322. doi:10.1080/11263500903374625.

Piirainen M, Liebisch O, Kadereit G. 2017. Phylogeny, biogeography, systematics and taxonomy of Salicornioideae (Amaranthaceae/ Chenopodiaceae) - a cosmopolitan, highly specialized hygrohalophyte lineage dating back to the Oligocene. Taxon. 66: 109-132. doi:10.12705/661.6.

Pinto-Carrasco D, Scheunert A, Heubl G, Rico E, Martínez-Ortega M. 2017. Unravelling the phylogeny of the root-hemiparasitic genus Odontites (tribe Rhinantheae, Orobanchaceae): evidence for five main lineages. Taxon. 66: 886-908. doi:10.12705/664.6.

Polatschek A. 1999. Flora von Nordtirol, Osttirol und Vorarlberg. Band 2: Samenpflanzen: Brassicaceae bis Euphorbiaceae [Flora of North Tirol, East Tirol and Voralberg. Volume 2: Spermatophytes: Brassicaceae to Euphorbiaceae]. Innsbruck: Tiroler Landesmuseum Ferdinandeum. German.

Poldini L. 2009. Guide alla Flora - IV. La diversità vegetale del Carso fra Trieste e Gorizia. Lo stato dell'ambiente. Le guide di Dryades 5 - Serie Florae IV (F - IV). Trieste: Ed. Goliardiche.

Poldini L, Oriolo G, Vidali M. 2001. Vascular flora of Friuli-Venezia Giulia. An annotated catalogue and synonymic index. Stud Geobot. 21: 3-227.

PPG I. 2016. A community-derived classification for extant lycophytes and ferns. J Syst Evol. 54: 563-603. doi:10.1111/jse.12229.

Raab-Straube E von, Henning T, Aedo C, Aghababyan M, Ardenghi NMG, Banfi E, Berendsohn WG, Brautigan S, Castroviejo S, Christenhusz M, et al. 2016. Sisyphos close to the mountain top: Euro+Med PlantBase is nearing its completion. Conference: XV OPTIMA Meeting, 6-11 June 2016, Montpellier, France. doi:10.13140/RG.2.1.4121.8803.

Rankou H, Culham A, Jury SL, Christenhusz MJM. 2013. The endemic flora of Morocco. Phytotaxa. 78: 1-69. doi:10.11646/phytotaxa.78.1.1.

Razifard H, Les DH, Tucker GC. 2017. Reticulate evolution in Elatine L. (Elatinaceae), a predominantly autogamous genus of aquatic plants. Syst Bot. 42: 87-95. doi:10.1600/036364417X694610.

Rešetnik I, Frajman B, Schönswetter P. 2016. Heteroploid Knautia drymeia includes $K$. gussonei and cannot be separated into diagnosable subspecies. Am J Bot. 103: 1-14. doi:10.3732/ajb.1500506.

Reveal JL. 2011. Summary of recent systems of angiosperm classification. Kew Bull. 66: 5-48.

Rilke S. 1999. Revision der Sektion Salsola s.l. der Gattung Salsola (Chenopodiaceae). Biblioth Bot. 149: 1-190.

Roma-Marzio F, Astuti G, Peruzzi L. 2015. Taxonomy, typification and karyology of Crepis lacera (Asteraceae). Phytotaxa. 208: 45-54. doi:10.11646/phytotaxa.208.1.4.

Roma-Marzio F, Bedini G, Müller J, Peruzzi L. 2016a. A critical checklist of the woody flora of Tuscany (Italy). Phytotaxa. 287: 1-134. doi:10.11646/ phytotaxa.287.1.1.

Roma-Marzio F, D’Antraccoli M, Astuti G, Maccioni S, Amadei L, Peruzzi L. 2018. Typification of the names in Trifolium L. described by Gaetano Savi. Taxon. 67. 
Roma-Marzio F, D'Antraccoli M, Astuti G, Maccioni S, Peruzzi L. 2016b. Neotypification of the name Rosa agrestis (Rosaceae). Phytotaxa. 284: 296-298. doi:10.11646/phytotaxa.284.4.7.

Roma-Marzio F, Najar B, Alessandri J, Pistelli L, Peruzzi L. 2017. Taxonomy of prickly juniper (Juniperus oxycedrus group): a crucial contribution at the meeting point of two cryptospecies. Phytochemistry. 141: 48-60. doi:10.1016/j.phytochem.2017.05.008.

Roma-Marzio F, Peruzzi L. 2017. Typification of the name Polygala flavescens DC. (Polygalaceae). Phytotaxa. 331: 299-300. doi:10.11646/ phytotaxa.331.2.15.

Romolini R, Souche R. 2012. Ophrys d'Italia. Société Occitane d'Orchidologie [The genus Ophrys in Italy. Occitan Society of Orchidology]. Saint-Martinde-Londres: Editions Sococor. Italian.

Rosati L, Romano VA, Bartolucci F, Bernardo L, Bouvet D, Cancellieri L, Caruso G, Conti F, Faraoni F, Banfi E, et al. 2017. Contribution to the floristic knowledge of the Maddalena Mountains (Basilicata and Campania, southern Italy). Ital Bot. 3: 73-82. doi:10.3897/ib.3.12519.

Rossi G, Montagnani C, Gargano D, Peruzzi L, Abeli T, Ravera S, Caruso G, Conti F, Faraoni F, Banfi E, et al., editors. 2013. Lista Rossa della Flora Italiana. 1. Policy Species e altre specie minacciate. Comitato Italiano IUCN e Ministero dell'Ambiente e della Tutela del Territorio e del Mare [Policy species and other threatened species. IUCN Italian Committee. Ministry of the Environment and Protection of Land and Sea]. Rome: Stamperia Romana. Italian.

Rossi G, Montagnani C, Abeli T, Gargano D, Peruzzi L, Fenu G, Magrini S, Gennai M, Foggi B, Wagensommer RP, et al. 2014. Are red lists really useful for plant conservation? The new red list of the Italian flora in the perspective of national conservation policies. Plant Biosyst. 148: 187-190. doi:10.1080/11263504.2013.868375.

Rossi G, Orsenigo S, Montagnani C, Fenu G, Gargano D, Peruzzi L, Wagensommer RP, Foggi B, Bacchetta G, Domina G, et al. 2016. Is legal protection sufficient to ensure plant conservation? The Italian Red List of policy species as a case study. Oryx. 50: 431-436. doi:10.1017/ S003060531500006X.

Saarela JM, Bull RD, Paradis MJ, Ebata SN, Peterson PM, Soreng RJ, Paszko B. 2017. Molecular phylogenetics of cool-season grasses in the subtribes Agrostidinae, Anthoxanthinae, Aveninae, Brizinae, Calothecinae, Koeleriinae and Phalaridinae (Poaceae, Pooideae, Poeae, Poeae chloroplast group 1). PhytoKeys. 87: 1-139. doi:10.3897/phytokeys.87.12774.

Salmeri C, Brullo C, Brullo S, Giusso Del Galdo G, Moysiyenko II. 2016. What is Allium paniculatum? Establishing taxonomic and molecular phylogenetic relationships within A. sect. Codonoprasum. J Syst Evol. 54: 123-135. doi:10.1111/jse.12170.

Santangelo A, Del Guacchio E, Cennamo P, Caputo P. 2017. Reassembling the Centaurea tenorei group (Asteraceae) puzzle: typification of the names. Phytotaxa. 298: 119-133. doi:10.11646/phytotaxa.298.2.2.

Schaefer $H$, Hechenleitner $P$, Santos-Guerra $A$, Menezes de Sequeira $M$, Pennington RT, Kenicer G, Carine MA. 2012. Systematics, biogeography, and character evolution of the legume tribe Fabeae with special focus on the middle-Atlantic island lineages. BMC Evol Biol. 12: 250. doi:10.1186/1471-2148-12-250.

Schüssler Ch, Freitag H, Koteyeva N, Schmidt D, Edwards G, Voznesenskaya E, Kadereit G. 2017. Molecular phylogeny and forms of photosynthesis in tribe Salsoleae (Chenopodiaceae). J Exp Bot. 68: 207-223. doi:10.1093/ jxb/erw432.

Schuster TM, Reveal JL, Bayly MJ, Kron KA. 2015. An uptated molecular phylogeny of Polygonoideae (Polygonaceae): relationships of Oxygonum, Pteroxygonum, and Rumex, and a new circumscription of Koenigia. Taxon. 64: 1188-1208. doi:10.12705/646.5.

Scoppola A, Lattanzi E. 2016. Remarks on the type material and the identity of the name Trifolium multistriatum W.D.J.Koch (T. vesiculosum aggr., Fabaceae). Phytotaxa. 277: 275-281. doi:10.11646/phytotaxa.277.3.5.

Scoppola A, Lattanzi E, Bernardo L. 2016. Distribution and taxonomy of the Italian clovers belonging to Trifolium sect. Vesicastrum subsect. Mystillus (Fabaceae). Ital Bot. 2: 7-27. doi:10.3897/italianbotanist.2.10361.

Sennikov AN. 2011. Chamerion or Chamaenerion (Onagraceae)? The old story in new words. Taxon. 60: 1485-1488.

Sennikov AN, Kurtto A. 2017. A phylogenetic checklist of Sorbus s.l. (Rosaceae) in Europe. Memoranda Soc Fauna Flora Fennica. 93: 1-78.
Shiels DR, Hurlbut DL, Lichtenwald SK, Monfils AK. 2014. Monophyly and Phylogeny of Schoenoplectus and Schoenoplectiella (Cyperaceae): evidence from chloroplast and nuclear DNA Sequences. Syst Bot. 39: 132-144. doi:10.1600/036364414X678198.

Soest JL van. 1969. Die Taraxacum-Arten der Schweiz [The genus Taraxacum in Switzerland]. Veröff Geobot Inst ETH Stiftung Rübel Zürich 42. German.

Sukhorukov AP. 2014. Карпология семейства Chenopodiaceae в связи с проблемами филогении, систематики и диагностики его представителей [The carpology of the family Chenopodiaceae in relations to problems of phylogeny, systematics and diagnostics of its representatives]. Tula: Grif i K.. 400 pp. Russian.

Soldano A. 1993. Trisetaria segetum (Savi) Soldano (Gramineae), nome corretto per Trisetaria parviflora (Desf.) Maire. Atti Soc Tosc Sci Nat, Mem, Ser B. 99: 15-21.

Soreng RJ, Peterson PM, Romaschenko K, Davidse G, Teisher JK, Clark LG, Barbera P, Gillespie LJ, Zuloaga FO. 2017. A worldwide phylogenetic classification of the Poaceae (Gramineae) II: an update and a comparison of two 2015 classifications. J Syst Evol. 55: 259-290. doi:10.1111/jse.12262.

Španiel S, Marhold K, Hodalova I, Lihova J. 2008. Diploid and tetraploid cytotypes of Centaurea stoebe (Asteraceae) in Central Europe: morphological differentiation and cytotype distribution patterns. Folia Geobot. 43: 131-158. doi:10.1007/s12224-008-9008-7.

Spaniel S, Marhold K, Passalacqua NG, Zozomova-Lihova J. 2011. Intricate variation patterns in the diploid-polyploid complex of Alyssum montanum-A. repens (Brassicaceae) in the Apennine peninsula: evidence for long-term persistence and diversification. Am J Bot. 98: 1887-1904. doi:10.3732/ajb.1100147.

Španiel S, Zozomová-Lihová J, Passalacqua NG, Marhold K. 2012. Infraspecific classification of Alyssum diffusum (Brassicaceae) in Italy. Willdenowia. 42 37-56. doi:10.3372/wi42.42104.

Stefanović S, Austin DA, Olmstead RG. 2003. Classification of Convolvulaceae: a phylogenetic approach. Syst Bot. 28: 791-806. doi:10.1043/02-45.1.

Stefanović S, Krueger L, Olmstead RG. 2002. Monophyly of the Convolvulaceae and circumscription of their major lineages based on DNA sequences of multiple chloroplast loci. Am J Bot. 89: 1510-1522. doi:10.3732/ajb.89.9.1510.

Štepánek J, Kirschner J. 2012. A taxonomic revision of Taraxacum sect. Erythrosperma (Compositae-Lactuceae) in Corsica. Feddes Repert. 123: 1-38. doi:10.1002/fedr.201200032.

Stöhr O. 2016. Liparis nemoralis (Orchidaceae), neu für Österreich, mit Anmerkungen zum Naturschutzwert des "Lavanter Forchachs" bei Lienz (Osttirol). Neilreichia. 8: 11-26.

Talavera S, García Murillo P, Smith H. 1986. Sobre el genero Zannichellia L. (Zannichelliaceae). Lagascalia. 14: 241-271.

The Plant List. 2013 onwards. The plant list. Version 1.1. http://www. theplantlist.org/.

Tison JM. 2001. Typification de Gagea cossoniana Pascher, de Gagea fragifera (Vill.) Ehr.Bayer \& G.López et de Gagea maroccana (A.Terracc.) Sennen \& Mauricio. Candollea. 56: 197-202.

Tison J-M, de Foucault B. 2014. Flora Gallica. Flore de France [Flora of France]. Mèze: Biotope Éditions. French.

Tison J-M, Peterson A, Harpke D, Peruzzi L. 2013. Reticulate evolution of the critical Mediterranean Gagea sect. Didymobulbos (Liliaceae), and its taxonomic implications. Plant Syst Evol. 299: 413-438.

Tomlinson PB, Posluzny U. 2001. Generic limits in the seagrass family Zosteraceae. Taxon. 50: 429-437. doi:10.2307/1223890.

Tondi G, Di Pietro R, Minutillo F. 2005. Contributo alla conoscenza del gen. Alchemilla L. (Rosaceae) nell'Appennino. Inform Bot Ital. 37: 380-381.

Troìa A, Azzella MM. 2013. Isoëtes sabatina (Isoëtaceae, Lycopodiophyta), a new aquatic species from central Italy. Plant Biosyst. 147: 1052-1058. doi :10.1080/11263504.2013.782902.

Troì A, Greuter W. 2014. Critical Flora of Italy: a critical conspectus of Italian Isoetes (Isoetaceae). Plant Biosyst. 148: 13-20. doi:10.1080/11263504.20 13.878409 .

Tropicos. 2017. Tropicos.org. Missouri Botanical Garden. http://www. tropicos.org.

Turner IM. 2017. From India to Madeira and back again: a new combination for a wide-ranging Spergularia (Caryophyllaceae). Willdenowia. 47: 213 216. doi:10.3372/wi.47.47303. 
Ubaldi D. 2016. Taxonomic remarks on Sesleria italica (Pamp.) Ujhelyi (Monocotyledones Poaceae). Quad Studi St Nat Romagna. 43: 117-131.

Uhlemann I. 2007. New species of the genus Taraxacum (Asteraceae, Cichorieae) from Croatia [Notulae ad floram euro-mediterraneam pertinentes 23]. Willdenowia. 37: 115-121. doi:10.3372/wi.37.37105.

Uhlemann I. 2017. The genus Taraxacum (Asteraceae, Cichorieae) sect. Erythrosperma in the northern coastal part of Croatia. Schlechtendalia. 32: 1-24.

Ujhelyi J. 1959. Révision des espèces du genre "Sesleria" en Italie [Revision of species of the genus "Ses/eria" in Italy]. Webbia. 14: 597-614. French.

Uribe-Convers S, Tank DC. 2016. Phylogenetic revision of the genus Bartsia (Orobanchaceae): disjunct distributions correlate to independent lineages. Syst Bot. 41: 1-13. doi:10.1600/036364416X692299.

Valdés B. 2011. Malvaceae. In: Greuter W, Raus Th, editors, Med-Checklist Notulae, 30. Willdenowia. 41:311-328. doi: 10.3372/wi.41.41213.

Valdés B, Rejdali M, Achhal El Kadmiri A, Jury SL, Montserrat JM. 2002. Catalogue des plantes vasculaires du nord du Maroc, incluant des clés d'identification [Catalogue of vascular plants from Northern Morocco, including identification keys]. 2 vols. Madrid: CSIC. French.

Vangjeli J. 2015. Exscursion flora of Albania. Königstein: Koeltz Scientific Books.

Villani M, Marcucci R, Peruzzi L. 2015. A note on the type of the name Rhizobotrya alpina (Brassicaceae). Phytotaxa. 236: 97-99. doi:10.11646/ phytotaxa.236.1.11.

Wagensommer RP, Bartolucci F, Fiorentino M, Licht W, Peccenini S, Perrino EV, Venanzoni R. 2017. First record for the flora of Italy and lectotypification of the name Linum elegans (Linaceae). Phytotaxa. 296: 161-170. doi:10.11646/phytotaxa.296.2.5.

Wagner F, Härtl S, Vogt R, Oberprieler C. 2017. "Fix Me Another Marguerite!": species delimitation in a group of intensively hybridizing lineages of ox- eye daisies (Leucanthemum Mill., Compositae-Anthemideae). Molec Ecol. 26: 4260-4283. doi:10.1111/mec.14180.

Wei R, Ebihara A, Zhu Y-M, Zhao C-F, Hennequin S, Zhang X-C. 2018. A totalevidence phylogeny of the lady fern genus Athyrium Roth (Athyriaceae) with a new infrageneric classification. Mol Phyl Evol 119: 25-36. doi:10.1016/j.ympev.2017.10.019.

Weigend M. 2006. Urtica dioica subsp. cypria, with a re-evaluation of the U. dioica group (Urticaceae) in Western Asia. Willdenowia. 36: 811-822. doi:10.3372/wi.36.36212.

Whittaker RJ, Araújo MB, Jepson P, Ladle RJ, Watson JEM, Willis kJ. 2005. Conservation biogeography: assessment and prospect. Divers Distr. 11: 3-23. doi:10.1111/j.1366-9516.2005.00143.x.

Wilson KL. 2017. Report of the General Committee: 20. Taxon. 66: 981. doi:10.12705/664.15.

Williams BRM, Mitchell TC, Wood JRI, Harris DJ, Scotland RW, Carine MA. 2014. Integrating DNA barcode data in a monograph of Convolvulus $L$. Taxon. 63: 1287-1306. doi:10.12705/636.9.

Wiegleb G, Bobrov A, Zalewska-Gałosz J. 2017. A taxonomic account of Ranunculus section Batrachium (Ranunculaceae). Phytotaxa. 319: 1-55. doi:10.11646/phytotaxa.319.1.1.

Wilson EO, Brown WL. 1953. The subspecies concept and its taxonomic application. Syst Zool. 2: 97-111.

Záveská Drábková L, Kirschner J. 2013. Oreojuncus, a new genus in the Juncaceae. Preslia. 85: 483-503.

Zozomová-Lihová J, Marhold K, Španiel S. 2014. Taxonomy and evolutionary history of Alyssum montanum (Brassicaceae) and related taxa in southwestern Europe and Morocco: diversification driven by polyploidy, geographic and ecological isolation. Taxon. 63: 562-591. doi:10.12705/633.18. 